\title{
Oral Abstract Sessions
}

\section{OAS 1 - Molecular approaches for novel AIT vaccines}

1

Coupling of grass pollen allergoids to mannan enhances immunogenicity and imprint dendritic cells with the capacity to generate Th1 immune responses with high levels of IL-10

Palomares, $\mathrm{O}^{1}$; Sirvent, $\mathrm{S}^{1}$; Soria, $\mathrm{I}^{2}$; Cases, $\mathrm{B}^{3}$; Díaz, ${\overline{\mathrm{C}^{4}}}^{4}$ Reche, $\mathrm{PA}^{4}$; López-Relaño, $\mathrm{J}^{4}$; Martínez-Naves, $\mathrm{E}^{4}$; Fernández-Caldas, $\mathrm{E}^{3}$; Subiza, $\mathrm{JL}^{2,3}$

${ }^{1}$ Complutense University of Madrid, Biochemistry and Molecular Biology I, Madrid, Spain; ${ }^{2}$ Hospital Clinico San Carlos, Immunology, Madrid, Spain; ${ }^{3}$ Inmunotek S.L., Alcalá de Henares, Spain; ${ }^{4}$ Complutense

University of Madrid, Immunology, Madrid, Spain

Background: The immunogenicity and efficacy of allergen vaccines highly depend on the capacity of dendritic cells (DCs) to capture them and to induce proper immune responses. The aim of this work was to compare the ability of DCs to internalize and mount immune responses to grass pollen $(\mathrm{N})$, glutaraldehyde-polymerised allergoids $(\mathrm{P})$ and glutaraldehyde-polymerised allergoids coupled to mannan (PM) extracts.

Method: Phleum pratense pollen extracts N, P and PM were from Inmunotek. PM was generated by innovative methods for coupling mannan to glutaraldehyde-modified allergens. Human monocytes were differentiated to DCs with IL-4/GM-CSF. Flow cytometry and confocal microscopy was used to determine allergen capture with and without specific blocking antibodies. Co-culture experiments, real-time quantitative PCR, ELISA and ELISPOT were performed. BALB/c mice were immunised with the different extracts and antibody levels in serum, proliferation of splenocytes and cytokine production were determined.

Results: PM showed enhanced capacity to be captured by human DCs than $\mathrm{N}$ or $\mathrm{P}$. The uptake mechanism was partially depending on receptor-mediated internalization involving mannose receptor and DC-SIGN. DCs stimulated with N,P and PM expressed high levels of HLA-DR, CD86 and CD83. PM-treated DCs produced significant higher levels of IL-6 and IL-10 than N- or P-treated DCs. Allogeneic and allergen-specific co-cultures showed that PM-treated DCs polarize T cells producing higher levels of IFNgamma and IL-10 and lower levels of IL-5 or IL-4 than N- or P-treated DCs. Splenocytes from mice immunised with PM showed higher proliferation rates than those from mice immunised with $\mathrm{N}$ or $\mathrm{P}$ and showed a Th1 cytokine-signature with high levels of IL-10.

Conclusion: Grass pollen allergoids conjugated to mannan show an enhanced immunogenicity and capacity to induce healthy $\mathrm{T}$ cell responses to allergens.

\section{2}

Immunological characterisation of a Pru p 3 proline variant for treatment of LTP-related peach allergy

Eichhorn, $\mathrm{S}^{1}$; Pablos, I ; Laimer, $\mathrm{J}^{1}$; Jensen, $\mathrm{BM}^{2}$; Versteeg, $\mathrm{S}^{3}$; Lackner, $\mathrm{P}^{1}$; Zuidmeer-Jongejan, $\mathrm{L}^{3}$; Fernandez-Rivas, $\mathrm{M}^{4}$; Papadopoulos, $\mathrm{N}^{5}$; Mari, $\mathrm{A}^{6}$; Poulsen, $\mathrm{LK}^{2}$; van Ree, $\mathrm{R}^{3}$; Ferreira, $\mathrm{F}^{1}$; Gadermaier, $\mathrm{G}^{1}$ ${ }^{1}$ Division of Allergy and Immunology, Department of Molecular Biology, University of Salzburg, Salzburg, Austria; ${ }^{2}$ Allergy Clinic, Copenhagen University Hospital Gentofte, Gentofte, Denmark; ${ }^{3}$ Department of

Experimental Immunology, Academic Medical Center, Amsterdam, The Netherlands; ${ }^{4}$ Hospital Clinico San Carlos, Facultad de Medicina-UCM, IdISSC, Madrid, Spain; ${ }^{5}$ National Kapodistrian University of Athens, Athens, Greece; ${ }^{6}$ Associated Centers for Molecular Allergology, Rome, Italy

Background: Adverse reactions to peach are highly prevalent in the Mediterranean area with persistent and potentially lifethreatening symptoms. We aimed to develop a safe and effective immunotherapeutic for treatment of peach allergy by targeting the major allergen Pru p 3.

Methods: Using in-silico mutagenesis a $4 \times$ proline variant of Pru p 3 was designed. Recombinant Pru p $3 \mathrm{C} 1$ was produced in E. coli using a pET-based expression system. The allergenic potential of the purified protein was compared to WT Pru p 3 using ImmunoCAP inhibition and basophil histamine release. To assess the immunogenicity, mice $(n=6)$ were subcutaneous immunised with Pru p 3 C1 (using WT Pru p 3 as a reference). Either aluminum hydroxide, or aluminum phosphate were used as adjuvants and mouse sera were analyzed for antibody reactivity. Additionally, splenocytes were tested for cytokine expression by proliferation assay and FACS analysis.

Results: Recombinant Pru p $3 \mathrm{Cl}$ was purified with a yield of $15 \mathrm{mg} / 1$ expression volume and physico-chemically characterised. Using sera of 26 DBPCFC-proven peach allergic patients, reduction in allergenic activity ranged from 10-fold to 5000 -fold (1000-fold on average) in ImmunoCAP inhibition or a biological histamine release assay. Immunisation with aluminum phosphate resulted in an enhanced humoral immune response compared to aluminum hydroxide in a mouse model. Blocking antibodies with IgG cross-reactivity to the wild-type molecule were induced in 3/6 animals. Reduced IL-4 and IL-5 levels were observed compared to WT Pru p 3.

Conclusion: Pru p 3 C1 shows strongly reduced IgE binding capacity while it maintained its immunogenicity. Therefore, this molecule can be regarded as a promising candidate for specific immunotherapy of peach allergy.

The study was supported by the FAST project EU grant 201871.

3

High throughput screening of mimotopes using OBOC combinatorial peptide library: in silico and in vitro validation of mimicry potential and specificity for allergy treatment

Leung, $\mathrm{NYH}^{1}$; Wai, $\mathrm{CYY}^{1} ; \mathrm{Ho} \mathrm{MHK}^{2}$; Lam, $\mathrm{K}^{3}$; Leung, $\mathrm{PSC}^{4}$; $\mathrm{Chu}, \mathrm{KH}^{1}$

${ }^{1}$ School of Life Sciences, The Chinese Univerisity of Hong Kong, Hong Kong City, Hong Kong; ${ }^{2}$ Department of Pediatrics and Adolescent Medicine, Queen Mary Hospital, The University of Hong Kong, Hong Kong City, Hong Kong: ${ }^{3}$ Department of Molecular Medicine, School of Medicine, University of California, Davis, CA, United States; ${ }^{4}$ Division of Rheumatology/Allergy, School of Medicine, University of California, Davis, CA, United States

Background: Mimotopes are novel candidates for immunotherapy based on their potential to induce blocking antibodies. However, conventional use of phage-displayed libraries to screen mimotopes are often time consuming. Here we present a novel method for rapid screening of 
mimotopes based on a One-Bead-OneCompound (OBOC) peptide library, using the shellfish allergen tropomyosin as a model. This method works well with unpurified serum from allergy patients and each round of screening could be completed in a week.

Method: OBOC library involves synthesis of million peptides on beads such that each bead has a single peptide entity. We used a two-stage screening approach with a alkaline-phosphatase (AP)-BCIP system. Beads reacting to $\mathrm{AP}$ and $\mathrm{BCIP}$ were removed prior to incubation with serum from allergy patients to eliminate non-specific binding. The reactive beads which turned into a turquoise color were isolated using a pipette under the microscope. The positive beads could be re-screened to maximize specificity.

Results: 25 mimotopes were identified after ten rounds of screening and analyzed with an epitope mapping program. Eight mimotopes with highest sequence identity with tropomyosin were conjugated to keyhole limpet hemocyanin for immunisation. Serum from immunised mice contained IgG specific to tropomyosin but not to the irrelevant allergen ovalbumin. Competitive inhibition ELISA also demonstrated the blocking potential of the $\mathrm{IgG}$ in inhibiting $\mathrm{IgE}$ binding of tropomyosin.

Conclusion: We demonstrated the use of OBOC library as an effective and rapid method for mimotope screening. This method is useful for allergens with a simple structure containing multiple epitopes. This is important in the design of immunotherapy as the blocking antibodies generated must be diverse enough to intercept most if not all IgE epitopes.

[This work is supported by a grant from Research Grants Council, HKSAR Government (CUHK463911)].

\section{4 \\ Cationic liposomes as drug-delivery system for sublingual immunotherapy in an OVA-induced allergic airway inflammation model}

Alija, $\mathrm{H}^{1}$; Rask, $\mathrm{CS}^{1}$; Brimnes, $\mathrm{J}^{1}$; Andresen, $\mathrm{TL}^{2}$ TALK-Abelló, Immunology, Hoersholm, Denmark; ${ }^{2}$ Micro- and Nanotechnology, Danish Technical University, Lyngby, Denmark

Background: Sublingual Immunotherapy (SLIT) is considered a safe and efficacious treatment for respiratory allergy. However, SLIT can cause adverse events and requires numerous allergen administrations during several years. A new delivery system, such as liposomes, could improve the delivery of allergen enabling the use of lower allergen concentration and hence increasing the safety profile. The objective of this study is to examine liposomes in an ovalbumin (OVA)-induced allergic airway inflammation model for their ability to improve delivery of antigens to oral mucosa compared with free OVA.

Method: OVA encapsulated into cationic 1-palmitoyl-2-oleoyl-phosphocholine

(POPC) liposomes were formulated using lipid-film hydration method. Liposomes were extruded and Dynamic Light Scattering was used to measure the size of the particles. Mice were treated sublingually with different frequencies and doses of encapsulated or free OVA, and subsequently sensitised to OVA by intraperitoneal injections followed by intra-nasal challenge with OVA during three days. Mice were sacrificed the following day and the eosinophils (EOS) in Bronco-Alveolar Lavage (BAL), allergen specific IgE in serum and Th2 cytokine responses were analyzed. POPC encapsulating liposomes were compared with free OVA.

Results: The size of the liposomes was measured to a diameter of $\sim 170 \mathrm{~nm}$ with a polydispersity index of $\sim 0.1$. SLIT with free as well as OVA encapsulated liposomes significantly reduced the percentages of eosinophils in BAL, and there was also a tendency to reduced levels of OVA-specific IgE in serum. No clear effects could be seen on cytokine production.

Conclusion: Prophylactic sublingual administration of OVA encapsulated liposomes was as good as free OVA in reducing allergic airway inflammation. Liposome formulations with other characteristics will be tested in the future in order to study whether the efficacy of SLIT could be improved.

\section{5}

Ligand effect on ultra-small nanoparticles for the immunomodulation of dendritic cells

Le Guevel, $\mathrm{X}^{1}$; Fernandez, $\mathrm{TD}^{2}$; Torres, $\mathrm{MJ}^{3}$; Blanca, $\overline{\mathrm{M}^{3} ; \text { Mayorga, }} \mathrm{C}^{2}$

${ }^{1}$ BIONAND, Andalusian Centre for Nanomedicine and Biotechnology, Therapeutic Nanosystem, Malaga, Spain; ${ }^{2}$ IBIMA, UMA, Research Laboratory, Regional University Hospital of Málaga, Malaga, Spain; ${ }^{3}$ IBIMA, UMA, Allergy Unit, Regional University Hospital of Malaga, Malaga, Spain

Background: Nanoparticles (NPs) have gained a tremendous interest over the last 10 years in the field of immunotherapy and particularly for allergen immunotherapy. One of the most successful strategies of using NPs for specific immunotherapy is based on targeting dendritic cells (DCs), which play a central role on the activation of the immune system. Nanoparticles offer the advantages to play the dual role of adjuvant and allergen delivery. They can be designed to either target or avoid interactions with the immune system to attain desirable immunostimulation or immunosuppression.

Method: In our study, we use gold nanoparticles as a model system to demonstrate the influence of physico-chemical parameters such as size and nature of the ligands to the inflammatory responses of human DCs. Thus, immature DCs were incubated with a series of gold NPs of two different sizes $(Æ \sim 1 \mathrm{~nm}$ and $12 \mathrm{~nm})$ with ligands containing different charges (methyl polyethyleneglycol and zwitterionic) on their surface. Their capacity to induce maturation and T-cell proliferation were investigated. Biodistribution of NPs in cells was determined by fluorescence microscopy.

Results: Results indicate a significant influence of the size and surface chemistry of NPs with different levels of DCs maturation, T-cell proliferation and cellular uptake. For instance, ultra-small particles with size $<2 \mathrm{~nm}$ protected by a ionic ligand like zwitterionic present low toxicity and could induce high maturation and high Tcell proliferation at very low concentration. By changing this ligand to a pegylated group, it was possible to reduce the maturation keeping a high $\mathrm{T}$-cell proliferation. Microscopy experiments confirm a higher cellular uptake with the zwitterionic ligand than the pegylated one and therefore are favorable to deliver allergen in a more efficient manner.

Conclusion: These data highlight the potential of using ultra-small particle to modulate the immune response of dendritic cells.

\section{6 Encapsulation of allergens into degradable nanocarriers \\ Köhring, $\mathrm{H}^{1,2,3}$; Bellinghausen, $\mathrm{I}^{1}$; Frey, $\mathrm{H}^{2}$; Saloga, $\mathrm{J}^{1}$ TDepartment of Dermatology, University Medical Center Mainz, Mainz, Germany; ${ }^{2}$ Institute of Organic Chemistry, Johannes Gutenberg University Mainz, Mainz, Germany; ${ }^{3}$ Graduate School Materials Science in Mainz, Mainz, Germany}

Background: Polymer nanoparticles are used to protect therapeutic proteins or drugs from degradation, obtaining higher local concentrations and enabling targeted transport. Encapsulation of allergens inside polymeric nanoparticles could also help to avoid antibody-mediated side effects occurring during allergen-specific immunotherapy.

Method: We have synthesized a novel type of difunctional, water soluble poly(ethylene glycol) dimethacrylate macromonomer with acetal-sites that degrade at acidic $\mathrm{pH}$. The allergen and the macromonomer were 
entrapped inside liposomes as templates that were produced by dual asymmetric centrifugation. Radical polymerization of the methacrylate groups inside the liposomes generated PEG-nanocarriers.

Results: The allergen-loaded nanocarriers were approximately $150-200 \mathrm{~nm}$ in size and showed low polydispersity indices. It could be demonstrated in in-vitro studies that immature dendritic cells (DC) internalize these protein-loaded, non-toxic PEG-nanocarriers. Viability and surface marker expression of DC was not affected by allergen-loaded nanoparticles. Furthermore, nanoparticles themselves did not lead to DC maturation. To investigate the targeted delivery of allergens into the antigen-presenting pathway, immature monocyte-derived DC from allergic donors were pulsed with Phleum pretense allergens (free or loaded into nanoparticles) and simultaneously matured with pro-inflammatory cytokines. Then, DC were co-cultured with autologous $\mathrm{CD}^{+} \mathrm{T}$ cells to induce proliferative responses. DC treated with allergen-loaded nanoparticles induced an allergen-specific proliferation reaching about $50 \%$ of the proliferation intensity observed for $\mathrm{T}$ cells stimulated with allergen-treated DC alone.

Conclusion: These data indicate that allergen-loaded nanoparticles are capable of inducing specific immune responses necessary for specific immunotherapy. Furthermore, this finding encourages functionalization of nanoparticles with targeting molecules like mannose for increased allergen uptake and presentation by DC. 


\section{Oral Abstract Session}

\section{OAS 2 - Allergy prevention and risk factors}

\section{7 \\ Maternal 25-hydroxyvitamin D (25(OH)D) levels in pregnancy and biomarkers of allergic airway disease in the offspring: results from 20 years follow-up}

Hansen, $S^{1}$; Maslova, $E^{1}$; Strøm, $M^{1}$; Cohen, $A^{2}$; Dahl, $\mathrm{R}^{3} ;$ Hoffmann, $\mathrm{HJ}^{4}$; Linneberg, $\mathrm{A}^{5}$; Olsen, $\mathrm{SF}^{1,6}$

${ }^{1}$ Department of Epidemiology Research, Statens Serum Institute, Center for Fetal Programming, Copenhagen, Denmark; ${ }^{2}$ Department of Clinical Biochemistry and Immunology, Statens Serum Institute, Copenhagen, Denmark; ${ }^{3}$ Odense University Hospital, Allergy Centre, Odense, Denmark; ${ }^{4}$ Department of Pulmonary Medicine, Institute for Clinical Medicine, Aarhus University, Aarhus, Denmark; ${ }^{5}$ Research Centre for Prevention and Health, Copenhagen University Hospital Glostrup, Glostrup, Denmark; ' ${ }^{6}$ epartment of Nutrition, Harvard School of Public Health, Boston, MA, United States

Background: Results from previous studies examining the association between prenatal levels of vitamin D and offspring asthma and allergy have been conflicting. Most studies have focused on outcomes in early childhood ( $<6$ years of age) and few studies have investigated long-term outcomes.

Objective: The objective of this study was to examine the association between maternal serum 25-hydroxyvitamin D $(25(\mathrm{OH})$ D) levels in gestational week 30 and markers of allergic airway disease in offspring at 20 years of age.

Method: We used data from a prospective birth cohort including 965 Danish pregnant women from 1988-1989. Maternal levels of $25(\mathrm{OH}) \mathrm{D}$ were quantified in serum by the LC-MS/MS method $(n=850)$. Offspring were invited for a clinical follow-up examination at age 20 ( $n=443,48 \%$ of 915 invited). Blood samples were analyzed for levels of eosinophil cationic protein (ECP), total immunoglobulin $\mathrm{E}(\operatorname{IgE})$, and specific $\operatorname{IgE}$ against 12 common allergens (ImmunoCAP) (allergic sensitisation $>0.35 \mathrm{kU}_{\mathrm{A}} / 1$ ). As a secondary outcome we included lung function measured by spirometry (Vitalograph) which included forced expiratory volume in the 1st second $\left(\mathrm{FEV}_{1}\right)$ and forced vital capacity (FVC). We used multivariable log-binomial or linear regression models to examine the association of maternal 25 $(\mathrm{OH}) \mathrm{D}$ with the outcomes.

Results: Mean (SD) concentration of maternal 25(OH)D was 80.4 (40.5) nM. We found no statistically significant associations for maternal levels of $25(\mathrm{OH}) \mathrm{D}$ with any of the outcomes. A $10 \mathrm{nM}$ increase in maternal $25(\mathrm{OH}) \mathrm{D}$ led to a relative change in offspring total $\mathrm{IgE}$ of $1.004 \mathrm{kU}_{\mathrm{A}} / 1(95 \%$ CI: $0.96-1.04)$, of $1.0003 \mu \mathrm{g} / 1(0.98-1.02)$ in ECP, $1.001 \mathrm{~L}(0.99-1.0004)$ in $\mathrm{FEV}_{1}$, and $1.002 \mathrm{~L}(0.99-1.004)$ in FVC. For a similar increase in maternal $25(\mathrm{OH}) \mathrm{D}$ the relative risk of offspring allergic sensitisation increased by $1.01(0.98-1.05)$.

Conclusion: We found no evidence of a relation between maternal levels of $25(\mathrm{OH})$ $\mathrm{D}$ in pregnancy and biomarkers of allergic airway disease in the offspring at 20 years of age.

\section{8}

Preventive allergen-specific sublingual immunotherapy in small children induces immunomodulation

Szépfalusi, $Z^{1}$; Bannert, $C^{1}$; Ronceray, $L^{2}$; Mayer, $E^{1}$; Hassler, $M^{1}$; Wissmann, $E^{1}$; Dehlink, $E^{1}$; Gruber, $S^{1}$; Graf, $A^{3}$; Valenta, $R^{4}$; Eiwegger, $\mathrm{T}^{1}$; Urbanek, $\mathrm{R}^{5}$ ${ }^{1}$ Pediatrics, Division of Ped Pulmonology, Allergy, Medical University of Vienna, Vienna, Austria; ${ }^{2}$ Pediatrics, St. Anna Children's Hospital, Vienna, Austria; ${ }^{3}$ Center for Medical Statistics, Informatics and Intelligent Systems, Medical University of Vienna, Vienna, Austria; ${ }^{4}$ Institute of Pathophysiology and Allergy Research, Medical University of Vienna, Vienna, Austria; ${ }^{5}$ Pediatrics, Medical Faculty, University of Freiburg, Freiburg, Germany

Background: Prevention of new IgE-sensitisations has been described during allergen-specific immunotherapy. However, prospective data using a preventive approach in very young children who would benefit most is missing. We performed an investigator-initiated, prospective, double blinded, placebo-controlled trial to investigate the safety, immunomodulatory and sensitisation-preventive effect of sublingual immunotherapy (SLIT) in mono/oligoclonally sensitised, clinically asymptomatic children at the age of $2-$ 5 years.

Method: In this double-blinded, randomized, placebo-controlled study, 31 mono/oligo-sensitised patients including house dust mite allergens or grass pollen (age 25 years) were included, who received SLIT to the respective source $(n=16)$ or placebo $(n=15)$. After dose up phase therapy was continued for 2 years. Parents recorded clinical events, vaccinations, drug intake in a diary. Skin prick testing, specific IgE and specific IgG testing was performed at baseline, 12 and 24 months. At the same time allergen specific proliferation (thymidine incorporation assay) and IL10- and TGFßdependent Treg function was measured via cytokine inhibition assays.

Results: Preventive application of SLIT in young children was safe (no relevant side effects in 21.170 single applications). After 12 and 24 months of treatment the rate of allergen-specific sensitisation (specific $\operatorname{IgE}$ and SPT reactivity) was comparable in the treatment and the placebo group. However, verum-treated patients displayed a significant up-regulation of allergen-specific IgG $(P<0.05)$. Furthermore, TGFß- and IL10-dependent inhibition $(P<0.05)$ was observed in vitro in the treatment group but not in the placebo group.

Conclusions: Preventive SLIT is safe in children at 2-5 years of age. Preventive SLIT induces regulatory mechanisms involving allergen-specific IgG, IL10 and TGFß. There is no evidence of an increase of IgE-sensitisation with preventive SLIT. Larger trials are needed to investigate the impact on the development of clinically relevant allergy.

\section{9}

Acinetobacter in the skin microbiota protects from atopic sensitisation

Fyhrquist, $\mathrm{NT}^{1}$; Ruokolainen, $\mathrm{L}^{2}$; Suomalainen, $\mathrm{A}^{1}$; Lehtimäki, $\mathrm{S}^{3}$; Veckman, $\mathrm{V}^{1}$; Vendelin, $\mathrm{J}^{1}$; Karisola, $\mathrm{P}^{1}$; Savinko, $T^{1}$; Lehto, $\mathrm{M}^{1}$; Jarva, $\mathrm{H}^{2}$; Kosunen, $\mathrm{T}^{2}$; Corander, $\mathrm{J}^{2}$; Petri, $\mathrm{A}^{2}$; von Hertzen, $\mathrm{L}^{2}$; Laatikainen, $\mathrm{T}^{4}$; Mäkelä, $\mathrm{M}^{5}$; Haahtela, $\mathrm{T}^{5}$; Greco, $\mathrm{D}^{1}$; Hanski, $\mathrm{I}^{2}$; Alenius, $\mathrm{H}^{1}$

${ }^{1}$ Finnish Institute of Occupational Health, Helsinki, Finland; ${ }^{2}$ University of Helsinki, Helsinki, Finland; ${ }^{3}$ University of Turku, Turku, Finland; ${ }^{4}$ National Institute for Health and Welfare, Helsinki, Finland; ${ }^{5} \mathrm{Helsinki}$ University Central Hospital, Helsinki, Finland

Background: Human commensal microbiota interacts with the immune system, and the outcome may depend on the immune status of the individual. In a previous study, we found that the relative abundance of Acinetobacter (Gammaproteobacteria) on the skin is positively correlated with the expression of the anti-inflammatory cytokine IL-10 by peripheral blood monocytes (PBMCs) in healthy (but not in atopic) individuals, suggesting a protective role for these bacteria on the skin. Here, building upon this observation, we analyze 
in detail the skin microbiota, atopic sensitisation, and immune function in a cohort of adolescents, as well as the influence of $\mathrm{Aci}$ netobacter on immune responses in vitro and in vivo.

Method: The skin microbiota of the study subjects was identified by $16 \mathrm{~S}$ rRNA sequencing and PBMCs were analyzed for baseline and allergen stimulated mRNA expression. Human monocyte derived dendritic cells and primary keratinocytes were incubated with $A$. lwoffii, and mice were injected intradermally with $A$. lwoffii during the sensitisation phase of the asthma protocol, followed by read-out of inflammatory parameters.

Results: In healthy (but not atopic) individuals, the relative abundance of Acinetobacter was associated with the expression of anti-inflammatory molecules by PBMCs. Moreover, the healthy individuals exhibited a robust balance between anti-inflammatory and Th1/Th2 gene expression, which was related to the composition of the skin microbiota. In cell assays and mouse models, Acinetobacter induced strong Th1 and anti-inflammatory responses by immune cells and skin cells, and protected from atopic sensitisation and lung inflammation via the skin.

Conclusion: The results suggest that skin commensals play a key role in tuning the balance of Th1, Th2, and anti-inflammatory responses to environmental allergens.

\section{0 \\ Altered $\mathrm{T}$ cell polarizing cytokine release of human dendritic cells by combinations of allergy-protective cowshed bacteria}

Störmer, $\mathrm{A}^{1}$; Stein, $\mathrm{K}^{1}$; Holst, $\mathrm{O}^{2}$; Heine, $\mathrm{H}^{1}$

Forschungszentrum Borstel, Innate Immunity, Borstel, Germany; ${ }^{2}$ Forschungszentrum Borstel, Structural

Biochemistry, Borstel, Germany

Background: Numerous epidemiological studies confirmed the protective effect of early-life contact to farming environment with respect to a later development of allergic diseases. Certain bacterial species Acinetobacter lwoffii F78, Lactococcus lactis G121 and Staphylococcus sciuri W620 have been isolated from cowsheds and their allergy-protective effects have been proven in various mouse models of acute allergic inflammation. However, the individual mechanisms of protection as well as the bacterial compounds involved appear to be different in each strain. Recently, Ege et al. could show that the protective effect of the farm environment correlates with its microbial diversity. Hence, we aimed to identify the immunological basis and mechanisms that lead to the enhanced allergy-protection.
Method: Dendritic cells are key players to polarize naïve $\mathrm{T}$ cells. Thus, we compared the allergy-protective activity of the individual bacterial strains with different combinations of all three strains on human monocyte-derived dendritic cells (huDC). In particular, we investigated the secretion of several cytokines focussing on $\mathrm{T}$ helper cell polarization by means of ELISA.

Results: IL-12, a strong Th1-polarizing cytokine, is highly induced by L. lactis, however, it is markedly reduced when high concentrations of either A. lwoffii or S. sciuri alone or both together were given simultaneously. In contrast, IL-10 as a cytokine more involved in regulatory $\mathrm{T}$ cell responses, appears to be never less induced by the combination of the bacteria than by the individual strains. A similar finding can be observed regarding the release of IL- 6 . Using these three bacterial strains simultaneously leads only under certain combinations to a synergistic activation of DCs.

Conclusion: Overall, the simultaneous stimulation with the abovementioned cowshed isolates revealed a number of different dependencies between the investigated cytokine and the combination and concentration of the stimulating strains, which could be the basis for the understanding of the enhanced allergy-protection and microbial diversity in the farming environment.

11

Allergy and autoimmunity following solid organ transplantation - prevalence, natural history and risk factors

Marcus, $\mathrm{N}^{1}$; Amir, $\mathrm{A}^{2}$; Grunebaum, $\mathrm{E}^{1}$; Dipchand, $\mathrm{A}^{3}$;

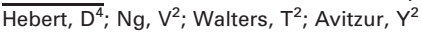
${ }^{1}$ Division of Allergy and Clinical Immunology, Department of Pediatrics, The Hospital for Sick Children, University of Toronto, Toronto, ON, Canada; ${ }^{2}$ Division of Gastroenterology, Hepatology and Nutrition, Department of Pediatrics, The Hospital for Sick Children, University of Toronto, Toronto, ON Canada; ${ }^{3}$ Division of Cardiology, Department of Pediatrics, The Hospital for Sick Children, University of Toronto, Toronto, ON, Canada; ${ }^{4}$ Division of Nepherology, Department of Pediatrics, The Hospital for Sick Children, University of Toronto, Toronto, ON, Canada

Background: Allergic and autoimmune diseases, have been sporadically reported in pediatric recipients of solid organ transplantation and commonly lead to significant morbidity and rarely, mortality. Data regarding their prevalence, natural course and risk factors is limited and has not been studied systematically. Therefore, we conducted a cross-sectional retrospective study to assess these parameters.

Methods: All children ( $<18$ years) who underwent liver, heart, kidney or intestinal transplantation at a pediatric tertiary medical center between 2000-2012, with a follow-up period of 6 months or more post transplant were included. Patients with a pre-transplant history of allergy or autoimmunity were excluded.

Results: 273 patients (111 liver recipients, 103 heart, 52 kidney, and seven multiple organs) with a median follow-up period of 3.6 years were included in the study. A total of $92(34 \%)$ patients developed allergy or autoimmune diseases (140 separate events) after transplantation with a high prevalence among liver $(41 \%)$ and heart transplant recipients $(40 \%)$ compared to kidney recipients $(4 \% ; P<0.001)$. Posttransplant allergies included eczema $(n=44)$, food allergy (22), eosinophilc gastrointestinal disease (11) and asthma (28). Autoimmunity occurred in $20(7.3 \%)$ patients, mainly autoimmune cytopenia $(n=10)$. In a multivariate analysis, female gender, young age at transplantation, family history of allergy, EBV infection and elevated eosinophil count more than 6 months post transplantation were associated with an increased risk for immune dysregulation. Two patients $(0.7 \%)$ died from autoimmune hemolytic anemia. In 50 out of the 140 events $(36 \%)$ the allergy or autoimmunity did not improve overtime.

Conclusions: Allergy and autoimmunity after solid organ transplantation are common in pediatric liver and heart recipients. Allergy and autoimmunity pose a significant health burden on transplant recipients and suggest a state of immune dysregulation post transplant.

12

The influence of family size and birth order on intracellular interferon (IFN)gamma production by cord blood mononuclear cells

Tereshchenko, $\mathrm{S}^{1}$; Smirnova, $\mathrm{S}^{1}$; Novitckii, I ${ }^{1}$; Tskhay, $\mathrm{V}^{2}$ 'Department of Child's Physical Health, Scientific Research Institute of Medical Problems of the North, Krasnoyarsk, Russian Federation; ${ }^{2}$ Krasnoyarsk State Medical University, Krasnoyarsk, Russian Federation

Background: Family size and birth order are inevitably correlated and both reduce atopy forming ('sibling effect'). Some studies suggest that the 'sibling effect' start in utero rather than during the postnatal period, but the immunological mechanisms of this connection are not known.

Aim: We supposed the influence of family size and birth order on IFN-gamma production by cord blood mononuclear cells (CBMC).

Method: Intracellular IFN-gamma expression as well as early activation marker CD69 (separate for total mononuclear cells population and CD4 + lymphocytes) was examined using flow cytometry after PHA stimulation of CBMC obtained from 89 
full-term newborns. Statistical analysis was performed using the Spearman's coefficient (r) and the Mann-Whitney U-test.

Results: Both factors, family size $(r=-0.21, \quad P=0.044)$ and birth order $(r=-0.25, P=0.018)$ were inversely correlated with absolute count of nonactivated CBMC, producing IFN-gamma (IFNgamma +/CD69- subtype). But only family size was positively correlated with activated
Th1 lymphocytes (CD4 + /IFN-gamma + (CD69 + subtype) $-r=0.22, P=0.038$. In neonates from mothers with family size $>2$ we have found the increasing levels of CD4 + /IFN-gamma,$\quad P=0.039$ and $\mathrm{CD} 4+/$ IFN-gamma $+/ \mathrm{CD} 69+\mathrm{CBMC}$, $P=0.035$.

Conclusion: Thus, bigger family size is associated with large amount of CBMC with IFN-gamma production. As family size has been hypothesized to be an indicator of more frequent exposure to infections, we suppose that infectious factor may act prenatal affecting Th1/Th2 balance through IFN-gamma production activation already on the moment of infant's birth. 


\section{Oral Abstract Session}

\section{OAS 3 - Novel diagnostic tools}

\section{3 \\ Identification and characterisation of Amb a 11, a new major allergen from the pollen of short ragweed (Ambrosia artemisiifolia)}

Nony, $\mathrm{E}^{1}$; Bouley, $\mathrm{J}^{1}$; Le Mignon, $\mathrm{M}^{1}$; Couret, $\mathrm{M}-\mathrm{N}^{1}$; Bussières, $L^{1}$; Groeme, $R^{1}$; Lautrette, $A^{1}$; Mariano, $S^{1}$; Baron-Bodo, $\mathrm{V}^{1}$; Bordas, $\mathrm{V}^{1}$; Chabre, $\mathrm{H}^{1}$; Koivu, $\mathrm{K}^{2}$; Batard, $\mathrm{T}^{1}$; Moingeon, $\mathrm{P}^{1}$

${ }^{1}$ Stallergenes, R\&D, Antony, France; ${ }^{2}$ Locusia Oy, Helsinky, Finland

Background: Allergy to pollen from short ragweed (Ambrosia artemisiifolia) is a severe and quickly expanding pollinosis across central Europe and Northern America. As of today, Amb a 1 is the only major allergen identified in ragweed pollen. Herein, we report on the identification of Amb a 11, as a new major allergen of diagnostic and therapeutic interest.

Method: In order to identify undescribed allergens in ragweed pollen, we performed gel electrophoresis followed by IgE-immunoblots using sera from 92 ragweed pollenallergic donors. Mass spectrometry (MS) in combination with molecular biology approaches, including PCR cloning and ragweed pollen transcriptome analysis, was used to identify IgE-binding proteins.

Results: IgE-immunoblotting indicated that over $54 \%$ of tested ragweed pollenallergic patients have IgE directed towards a so far undescribed $37-\mathrm{kDa}$ protein, thus established as a major allergen. Following gene cloning as well as extensive immunological and MS characterisation of both natural and recombinant forms expressed in E. coli or tobacco plants, this new allergen termed Amb a 11 (GenBank KF528831) was shown to consist of a 262amino acid-long protein related to papain. Molecular modelling revealed a strong structural homology with cysteine proteases known as allergens, such as the house dust mite-derived allergen Der p 1.

Conclusion: We identified a previously undescribed major allergen from short ragweed pollen. This allergen was officially recognised as Amb a 11 by the World Health Organization and International Union of Immunological Societies (WHO/ IUIS) Allergen Nomenclature Sub-committee (http://www.allergen.org/viewallergen. php?aid =784). Because of its involvement in the $\operatorname{IgE}$ sensitisation of most ragweed allergic patients, and given its homology with cysteine proteases known to represent clinically important allergens, we conclude that Amb a 11 is of great diagnostic and therapeutic value.

14

Component-resolved diagnosis: identification and purification of new low molecular weight lupine allergens

Jappe, $\mathrm{U}^{1,2}$; Hellmig, $\mathrm{S}^{1}$; Warneke, $\mathrm{D}^{1}$; Riffelmann, $\mathrm{FW}^{3}$; Lange, $\mathrm{L}^{4}$; Abraham, $\mathrm{S}^{5}$; Hompes, $\mathrm{S}^{6}$; Reese, $\mathrm{G}^{7}$; Becker, WM $^{1}$; Mari, $\mathrm{A}^{8}$; Petersen, $\mathrm{A}^{1}$

Division of Clinical and Molecular Allergology, Research Center Borstel, Borstel, Germany;

${ }^{2}$ Department of Dermatology, University of Luebeck, Luebeck, Germany; ${ }^{3}$ Department of Allergology, Fachkrankenhaus Kloster Grafschaft, Schmallenberg, Germany; ${ }^{4}$ Department of Pediatrics, St. MarienHospital, Bonn, Germany; ${ }^{5}$ Department of Dermatology, University of Dresden, Dresden, Germany; ${ }^{6}$ Department of Dermatology and Allergy, Charité Campus Mitte, Berlin, Germany; ${ }^{7}$ Allergopharma Joachim Ganzer KG, Reinbeck, Germany; ${ }^{8}$ Associated Centers for Molecular Allergology, Rome, Italy

Background: Lupine flour is a valuable food due to its favourable nutritional properties. Although the allergenic potential and severe reactions have been reported, its use is increasing. Cross-reactivity between lupine and other legumes with and without clinical relevance, particularly peanut, is well known. So far, only one lupine allergen was documented by the IUIS allergen nomenclature committee. Additional reports exist on conglutins being relevant. Since for peanut species differences in allergen content have been described, it had to be clarified whether the three different Lupinus species used for nutrition, differ and whether well known legume allergens associated with anaphylaxis exist in lupine seed as well. The aim of this study was to identify new lupine allergens with a focus on hydrophobic and low molecular weight (LMW) proteins.

Method: The sensitisation profile of lupine allergic patients was obtained by IgE detection via CAP-FEIA. Protein, DNA and EST data bases were queried for lupine proteins homologous to already known legume allergens. Sera from peanut allergic patients were used to investigate for crossreactivity. Acidic extracts from L. angustifolius and L. luteus were examined by SDS-PAGE and screened by immunoblot- ting for IgE-binding proteins. Furthermore, the extracts were separated by gel filtration and ion exchange chromatography. IgE-reactive LMW-components underwent $\mathrm{N}$-terminal sequencing for identification. Immunoblot analysis with lipid transfer protein (LTP)-reactive sera from peanut allergics was positive. Afterwards, sera of patients with lupine allergy were used to demonstrate species-specific $\operatorname{IgE}$ reactivity as well.

Results: The investigation of the extracts of different lupine species revealed differences regarding the concentration of certain IgE-binding proteins. Focussing on LMW proteins, a $10 \mathrm{kDa}$ component was confirmed via $\mathrm{N}$-terminal sequencing to be an LTP, which was detected by $\operatorname{IgE}$ in sera of patients with lupine and peanut allergy. Conclusion: Lupine seeds of different Lupinus species vary qualitatively and quantitatively in their allergen compositions. LTPs purified from the natural source of two different lupine species were IgE-reactive. From L. luteus, additional low molecular weight proteins could be isolated, without revealing $\operatorname{IgE}$ reactivity at present.

15

Agreement between skin tests and specific IgE detection by components through conventional technique and microarray against panallergens and cross reactive carbohydrate determinants

Martínez-Aranguren, $\mathrm{RM}^{1}{ }^{1}$; Goikoetxea, $\mathrm{MJ}^{1}$; Terrados, $\mathrm{S}^{2}$; García, $\mathrm{BE}^{3}$; Blanca-López, $\mathrm{N}^{4}$; Parra, $\mathrm{A}^{5}$; Sanz, $\mathrm{ML}^{1}$ ${ }^{1}$ Clínica Universidad de Navarra, Allergology and Clinical Immunology, Pamplona, Spain; ${ }^{2}$ Hospital Universitario Ramón y Cajal, Allergy Service, Madrid, Spain; ${ }^{3}$ Complejo Hospitalario de Navarra, Allergy Service, Pamplona, Spain; ${ }^{4}$ Hospital Infanta Leonor, Allergy Service, Madrid, Spain; ${ }^{5}$ Complexo Hospitalario Universitario A Coruña, Allergy Service, A Coruña, Spain

Sensitisation to panallergens and to cross reactivity carbohydrate determinants $(\mathrm{CCD})$ is frequent in pollen and/or vegetable allergic patients.

In a sample of 433 pollen and/or vegetable foods allergic patients we analysed the prevalence of sensitivity to lipid transfer proteins (LTP), PR10 proteins, profilins, polcalcins and CCD by skin tests to peach extract enriched with Pru p $3(n=206$; $47.5 \%)$, birch extract $(n=133 ; 30.7 \%)$, 
palm tree profilin $(n=115 ; 26.5 \%)$, palm tree polcalcin $(n=86 ; 19.8 \%)$ and horseradish peroxidase $(n=46 ; 10.6 \%)$ respectively. We also analysed the agreement strength by Kappa Index (K) among the results obtained with skin tests and two specific IgE quantification methods (ImmunoCAP and ISAC 112) for the panallergens Pru p 3, Bet v 1, Bet v 2 and Phl p 7, and for MUXF3 as CCD marker.

The LTP results obtained very good agreement between the three methods, skin prick test, ISAC and CAP ( $\mathrm{K}=0.69$; 0.71; 0.76). The agreement between skin test to birch and sIgE to Bet $\mathrm{V} 1$ was poor $(\mathrm{K}=0.15 ; \quad 0.13)$, whereas agreement between both $\mathrm{sIgE}$ determinations was very good $(\mathrm{K}=1.0)$. The results of skin tests to palm tree profilin and the results of $\operatorname{sIgE}$ to Bet $\mathrm{v} 2$ had good agreement $(\mathrm{K}=0.69$; 0.63 ) and very good agreement between both $\operatorname{sIgE}$ determinations $(\mathrm{K}=0.85)$. The agreement between skin tests to polcalcin and $\mathrm{sIgE}$ determinations to $\mathrm{Phl} \mathrm{p} 7$ was weak $(K=0.29 ; 0.31)$, and so was the agreement between skin test to peroxidase and $\operatorname{sIgE}$ to MUXF3 $(\mathrm{K}=0.39 ; 0.30)$, whereas the sIgE determinations to $\mathrm{Phl} \mathrm{p} 7$ had very good agreement $(K=0.85)$, and so did the SIgE determinations to MUXF3 $(\mathrm{K}=0.86)$.

We also analyzed the correlation between the quantitative values obtained with ImmunoCAP and ISAC 112 was good for Bet $\mathrm{v} 1\left(\mathrm{r}_{\mathrm{s}}=0.51\right)$ and Phl p 7 $\left(r_{\mathrm{s}}=0.65\right)$ and very good to Pru p 3 $\left(\mathrm{r}_{\mathrm{s}}=0.83\right)$, Bet $\mathrm{v} 2(\mathrm{rs}=0.88)$ and MUXF3 $\left(r_{\mathrm{s}}=0.78\right)$.

We can conclude that the agreement between the results obtained with both sIgE quantification methods is very good, whereas the agreement of these techniques with skin tests is good for LTP and profilin, and weak for polcalcin, CCD and PR10 proteins.

\section{6 \\ Health economic benefit of including component resolved diagnostics (ImmunoCAP ISAC) in in vitro diagnostics algorithm in a prospective trial with suspected food allergic school children in Finland \\ Hermansson, L-L ${ }^{1}$; Pensamo, $E^{2}$; Korhonen, $\mathrm{K}^{3}$; Rantanen, $\mathrm{S}^{3}$; Isoaho, $\mathrm{R}^{4}$; Savolainen, $\mathrm{J}^{2}$ \\ ${ }^{1}$ Thermo Fisher Scientific, IDD, Uppsala, Sweden; ${ }^{2}$ Department of Allergology, University of Turku, Turku, Finland; ${ }^{3}$ Department of School and Preschool Health Care, Härkätie Primary Care, Lieto, Finland; ${ }^{4}$ Department of General Practice, University of Turku, Turku, Finland}

Background: The aim of the study is in accordance to the Finnish Allergy Program 2008-2018, to investigate the possibility to have avoidance diets to foods decreased by $50 \%$. In this study the primary focus is in the algorithm where in vitro diagnostics is included with patient history in primary care for school children. The health economic benefit of using component resolved diagnostics with ISAC ImmunoCAP is additionally evaluated.

Methods: Analysis from database at Härkätie Primary Care Unit including 2317 school children in Finland. Of these 199 children $(8.6 \%)$ are on avoidance diet exclusive celiac disease. Database indicates that, there is a milk free diet for 37 pupils $(1.6 \%)$ and egg free diet for 32 children $(1.4 \%)$. Peanut and hazelnut allergy is indexed together as 39 children. Database included six soya allergic children. History of anaphylaxis or severe reactions was noted in seven children. The avoidance recommendation is given to the schools by the parents and has a direct budget impact in the society and substantial impact on patient and family's quality of life.

Results: The 199 children that had official food restriction notification for the school kitchens were contacted by written letter. Of the suspected allergic children $36(18 \%)$ were interviewed by physician. Of those, 24 children agreed to take part in the study and having an in vitro diagnostics performed $(67 \%)$. An unnecessary diet was concluded for 15 children, which is $63 \%$ of children included in the study. The unnecessary diets were accordingly: cow's milk 7 $(29 \%)$, peanut $5(21 \%)$, egg $3(13 \%)$, fish 1 $(4 \%)$ and fruits $1(4 \%)$. The peanut was reintroduced to the children after component resolved diagnostics with ImmunoCAP ISAC evaluation. The egg and milk diets were reintroduced to the children after specific IgE IVD. Prior to the introduction of food a secondary care specialist food provocation was performed to confirm the IVD results.

Conclusions: ImmunoCAP ISAC IVD was beneficial in $54 \%$ of the cases and in $21 \%$ of the cases it was critical for decision making. The aim of the study was well reached as avoidance diets to foods decreased by $63 \%$. From the study results, even with low patient recruitment, there is a large likelihood that introducing ImmunoCAP ISAC IVD to traditional diagnostic algorithm can be considered costeffective, with an average cost per avoided unnecessary diet for 480 EURs per child. To confirm the findings a larger study will be executed.

\section{The MeDALL-chip, a new diagnostic tool} for allergic disease

Skrindo, ${ }^{1,2}$; Lupinek, $C^{3}$; Valenta, $\mathrm{R}^{3}$; Melen, $\mathrm{E}^{4}$; Hovland, $\mathrm{V}^{1,5}$; Carlsen, K-H ${ }^{1,5,6}$; Mowinckel, $\mathrm{P}^{5}$; Bousquet, $\mathrm{J}^{7,8}$; Anto, $\mathrm{J}^{9,10,11}$; Lødrup Carlsen, $\mathrm{KC}^{1,5}$

${ }^{1}$ Faculty of Medicine, University of Oslo, Oslo, Norway; ${ }^{2}$ Department of Otorhinolaryngology, Akershus University Hospital, Lørenskog, Norway; ${ }^{3}$ Division of Immunopathology, Infectiology, and Immunology, Medical University of Vienna, Vienna, Austria;

${ }^{4}$ Karolinska Institutet, Institute of Environmental Medicine, Stockholm, Sweden; ${ }^{5}$ Department of Pediatrics, Oslo University Hospital, Oslo, Norway; ${ }^{6}$ Norwegian School of Sport Sciences, Oslo, Norway; ${ }^{7}$ Department of Respiratory Diseases, University Hospital, Hôpital Arnaud de Villeneuve, Montpellier, France; ${ }^{8}$ Inserm, CESP Centre for Research in Epidemiology and Population Health, U 1018 , Respiratory and Environmental Epidemiology Team, Villejuif, France; ${ }^{9}$ Centre for Research in Environmental Epidemiology (CREAL), Barcelona, Spain; ${ }^{10}$ Municipal Institute of Medical Research (IMIM-Hospital del Mar), Barcelona, Spain; ${ }^{11}$ CIBER Epidemiologia y Salud Publica (CIBERESP), Barcelona, Spain

Background: To meet the challenge of increased allergic diseases, the EU funded MeDALL (Mechanisms of the Development of Allergy) project aims to generate novel knowledge on the mechanisms of initiation of allergy and to propose early diagnosis, prevention and targets for therapy. The role of specific immunoglobulin $\mathrm{E}$ (s-IgE) in allergic disease is not always clear. Multiple s-IgE analyses including those to recombinant allergens may provide new ways of assessing this relationship, thus a micro-array MeDALL-chip with 176 allergen components was developed. The aim of the present study was to investigate the precision of the MeDALLchip in asthma and allergic rhinitis in children.

Method: 265 children from the 'Environment and Childhood Asthma Study' were investigated at 10 and 16 years of age with interview, skin prick test (SPT), serologic tests of s-IgE by CAP and component analyses with the MeDALL-chip, including component analyses.

Results: Allergic sensitisation was surprisingly high measured by the MeDALL-chip. When using the inhalant allergens only, the MeDALL-chip was comparable to CAP/ SPT in relation to clinical illness, marked MeDALL-chip Inhalation, CAP Inhalation and SPT Inhalation (table 1).

Sensitivity was similar for CAP Inhalation and MeDALL-chip Inhalation, whereas specificity appeared better for CAP Inhalation. The negative predictive value (NPV) was high for both tests, whereas the positive predictive value (PPV) was generally low (table 2).

Conclusion: In these paired samples six years between investigations, allergic sensitisation increased by all three tests, were 
Table

\begin{tabular}{|c|c|c|c|c|c|c|c|c|}
\hline Table 1 (\%) & 10 years & 16 years & & 10 years & 16 years & & 10 years & 16 years \\
\hline $\begin{array}{l}\text { Any MeDALL } \\
\text { Chip }\end{array}$ & 60.0 & 75.8 & $\begin{array}{l}\text { Rhinitis and Any MeDALL } \\
\text { Chip Inhal }\end{array}$ & 12.5 & 26.0 & $\begin{array}{l}\text { Asthma and Any MeDALL } \\
\text { Chip Inhal }\end{array}$ & 4.5 & 8.3 \\
\hline Any CAP & 32.8 & 45.8 & Rhinitis and Any CAP Inhal & 12.5 & 25.4 & Asthma and Any CAP Inhal & 4.5 & 8.7 \\
\hline Any SPT & 25.5 & 48.6 & Rhinitis and Any SPT Inhal & 11.5 & 24.1 & Asthma and Any SPT Inhal & 3.4 & 7.3 \\
\hline Table 2 (\%) & Age & Disease & Sensitivity & Specificity & PPV & NPV & & \\
\hline \multirow{2}{*}{$\begin{array}{l}\text { MeDALL-chip } \\
\text { Inhal }\end{array}$} & 10 years & Rhinitis & 80.5 & 71.0 & 33.7 & 95.2 & & \\
\hline & 16 years & Rhinitis & 83.1 & 65.9 & 52.7 & 89.6 & & \\
\hline \multirow[t]{2}{*}{ CAP Inhalation } & 10 years & Rhinitis & 80.5 & 79.0 & 41.3 & 95.7 & & \\
\hline & 16 years & Rhinits & 81.7 & 72.0 & 56.8 & 89.7 & & \\
\hline
\end{tabular}

similar by SPT and CAP, but clearly higher with the MeDALL-chip. As for illness the MeDALL-chip was comparable to CAP for sensitivity and NPV. The MeDALL-chip may be useful in research in cohorts with small amounts of serum. The importance of $\operatorname{IgE}$ sensitisation in asymptomatic children as measured by the MeDALL-chip needs to be further investigated.

18

Evaluation of a new microarray based system for allergy testing

Palomba, $A^{1}$; Maccari, $M^{2}$; Baldracchini, $F^{2}$; Mazzoleni, $\mathrm{G}^{2}$; Crisanti, $\mathrm{A}^{2}$; Marcucci, $\overline{\mathrm{F}}^{1}$

${ }^{1}$ University of Perugia, Perugia, Italy; ${ }^{2}$ Microtest

Diagnostics, London, United Kingdom

Background: The Microtest Allergy Test (i.e. Microtest Biochips and Reagents) is a new concept in automated in vitro diagnostic (IVD) immunoassays for the determination of specific IgE in serum or plasma. The test was designed to work in conjunction with the Microtest instrument. The Microtest system is a walkaway technology designed for use in both clinical and physician office laboratories. Allergens immobilised on a biochip (microarray) react with specific IgE in the patient sample. The allergen- $\operatorname{IgE}$ immunocomplex is detected using an enzymatic amplification system. The fluorescense signal is then collected and analysed in a semi-quantitative format. Up to five patients samples can be processed in about four hours with less than 5 min hands-on time per run.

Method: In a recent study, 108 patients samples were run in parallel using the Microtest system and a commercially available IVD allergy immunoassay. Samples were tested in parallel for specific IgE against 15 allergens (e.g. natural extracts and components) on both platforms generating a total of 3240 test results, 1620 from each method.

Results: Qualitative and semi-quantitative analysis resulted in good agreement between the two platforms, $76 \%$ and $86 \%$ respectively. The two systems showed comparable results of positive and negative predictive values based on clinical history and SPT with the Microtest Allergy system producing lower number of false positive determinations. The Microtest Allergy test also showed a lower level of cross reactivity against both related and non-related allergens.

Conclusion: Overall the study showed that the performance of the Microtest system was comparable to the current allergy test systems, showing good clinical performance and diagnostic accuracy. 


\section{Oral Abstract Session}

\section{OAS 4 - Asthma genetics}

19

Association of season of birth with DNA methylation and allergic outcomes

Lockett, $\mathrm{GA}^{1}$; Soto-Ramírez, $\mathrm{N}^{2}$; Ray, $\mathrm{M}^{2}$; Zhang, $\mathrm{H}^{2}$; Rezwan, $\mathrm{Fl}^{1}$; Ewart, $\mathrm{SE}^{3}$; Arshad, $\mathrm{H}^{1,4}$; Karmaus, $\mathrm{W}^{2}$; Holloway, JW ${ }^{1}$

${ }^{1}$ Faculty of Medicine, University of Southampton, Southampton, United Kingdom; ${ }^{2}$ School of Public Health, University of Memphis, Memphis, TN, United States; ${ }^{3}$ Department of Large Animal Clinical Sciences, Michigan State University, East Lansing, MI, United States; ${ }^{4}$ The David Hide Asthma \& Allergy Research Centre, Newport, United Kingdom

Background: Season of birth can influence early-life development of allergy: children born in autumn or winter are more likely to develop allergic disease or atopy. In mammals maternal photoperiodic exposure can alter foetal development, and in the hamster this depends on reversible DNA methylation. Thus altered DNA methylation represents an alternative mechanism for the association between season of birth and risk of allergic disease. To test this, we examined the association of season of birth with DNA methylation.

Method: In a subset of 18 year old men and women from the Isle of Wight 1989 birth cohort ( $n=367$ ), genome-wide methylation of peripheral blood leukocytes was assessed at $>484000 \mathrm{CpG}$ sites using the Illumina $450 \mathrm{~K}$ array. A training-testing strategy was used to assess the association of methylation levels with season of birth.

Results: Summer-borns had increased risk of rhinitis at age 18 , relative to autumnborns $(\mathrm{RR}=1.76$; 95\% CI 1.14, 2.73). After controlling for maternal socioeconomic status, we detected 1557 CpGs whose methylation levels were associated with season of birth. Given the potential dependence between season of blood sample collection and season of birth, we separately identified $\mathrm{CpGs}$ associated with season of blood collection, and detected a set of 297 CpGs whose DNA methylation was influenced by season of both birth and blood collection. Finally, we examined the biological functions enriched within $\mathrm{CpGs}$ influenced by season of birth alone, and linked methylation to asthma, eczema and rhinitis status at age 18 .

Conclusion: Season of birth was associated with both allergic disease and DNA methylation, and some season-associated methylation sites also relate to allergic phenotypes.
These results suggest that season of birth may influence risk of allergic disease via DNA methylation. Our findings also highlight the importance of controlling for season of birth in large-scale epigenome-wide association studies for allergic disease.

\section{0}

Pre- and postnatal genetic influence on lung function development: the COPSAC $_{2000}$ prospective birth cohort

Kreiner-Møller, E; Bisgaard, H; Bønnelykke, K Copenhagen Prospective Studies on Asthma in Childhood, Health Sciences, University of Copenhagen, Danish Pediatric Asthma Center, Copenhagen

University Hospital, Copenhagen, Gentofte, Denmark

Background: A number of susceptibility genes for reduced lung function in adulthood have been identified in genome-wide association studies. We aimed to study the association of these candidate genetic variants with neonatal lung function and lung function development till age 7 years.

Method: Lung function measurement by spirometry (FEV0.5, FEF50) using the raised volume thoracoabdominal compression technique was assessed in 411 high-risk newborns from the Copenhagen Prospective Study on Asthma in Childhood 2000 (COP$\mathrm{SAC}_{2000}$ ) cohort. Bronchial responsiveness to metacholine challenge was measured assessing the provocative dose resulting in $15 \%$ drop in lung function (PD15). Lung function (FEV1, FEF50) and responsiveness (PD20, 20\% drop in lung function) measures were repeated at age 7 years. Genetic risk scores were calculated based on reported single nucleotide polymorphisms for adult lung function (FEV1/FVC, FEV1) as the number of risk alleles weighted on known effect size and associated to lung function measures as Z-scores at birth, age 7 and to the development to age 7 (FEV0.5/1, FEF50 and PD15/20).

Results: The genetic risk scores were not associated with lung function measures at age 1 month, but the FEV1/FVC genetic risk score was associated with FEF50 by age $7($ Beta $=-1.09, P=0.01)$ and similarly to the growth in FEF50 from birth to age 7 $($ Beta $=-0.97 ; P=0.02)$. This score was also associated with bronchial responsiveness (PD20) at age 7 (Beta $=-1.03$, $P=0.02)$ and change in responsiveness from birth to age $7 \quad($ Beta $=-0.90$, $P=0.05)$.

Conclusion: Lung function genetic variants identified in adults were not associated with neonatal lung function or bronchial responsiveness but with the development of these lung function measures during early childhood. This suggests a window of opportunity for interventions targeting these genetic mechanisms.

\section{1}

Folate status as a modifier of epigenetic profile in human neonatal $\mathrm{CD}^{+} \mathrm{T}$ cells

Harb, $\mathrm{H}^{1}$; Amarasekera, $\mathrm{M}^{2}$; Tulic, $\mathrm{MK}^{2,3}$; Pfefferle, $\mathrm{PI}^{1}$; Martino, $\mathrm{D}^{4}$; Renz, $\mathrm{H}^{1}$; Kesper, $\mathrm{DA}^{1}$; Prescott, $\mathrm{SL}^{2}$ ${ }^{1}$ Institute of Laboratory Medicine and

Pathobiochemistry, Molecular Diagnostics, Philipps Universität Marburg, Marburg, Germany; ${ }^{2}$ School of Paediatrics and Child Health, University of Western Australia, Perth, WA, Australia; ${ }^{3}$ Tolerance Immunitaire, Université de Nice Sophia-Antipolis, Hôpital de I'Archet, Nice, France; ${ }^{4}$ Murdoch Childrens Research Institute, Melbourne, Vic., Australia

Background: Normal immune development in early life is important for the protection against inflammatory diseases such as allergies. Changes in the dynamics of gene-environment interactions in early life are implicated in the rising of disease predisposition. Exposure to different environmental factors during pregnancy can play a very important role in the development of immune response. In the present study we sought to examine the effects of high in utero exposure to folate on neonatal epigenetic profiles of genes associated with allergic inflammation.

Method: 23 neonates were selected from a large prospective cohort including the two extremes of maternal folate levels. Different epigenetic modifications, including DNA methylation and histone acetylation were analyzed at allergy-associated genes. Furthermore, cytokine levels were measured following both polyclonal and allergen specific stimulation of cord blood mononuclear cells. Results: There was a bias toward a Th2 phenotype by looking at the whole cohort epigenetic profile. Histone acetylation on both $\mathrm{H} 3$ and $\mathrm{H} 4$ histones at the GATA3 locus and $\mathrm{H} 4$ acetylation at the IL9 locus was increased in children born to mothers with high folate levels together with decreased acetylation at the IFNG locus in the same group. Cytokine levels were not 
significantly differed between the two groups. Nevertheless, cytokine ratios of Th2/Th1 showed an increase in the high folate group.

Conclusion: Maternal folate as a known methyl donor is likely influencing the development of the fetal immune system by modifying epigenetic marks like histone acetylation. High folate levels render allergy-associated genes more permissive for transcription and might thus affect disease development later in life.

\section{2 \\ Genetic associations of the respone to single-high dose inhaled corticosteroid treatment in children with asthma exacerbation \\ Keskin, $\mathrm{O}^{1}$; Uluca, $\mathrm{U}^{2}$; Birben, $\mathrm{E}^{3}$; Coskun, $\mathrm{Y}^{2}$; Ozkars, $\mathrm{MY}^{1} ;$ Keskin, $\mathrm{M}^{2}$; Kucukosmanoglu, $\mathrm{E}^{1} ;$ Kalayci, $\mathrm{O}^{3}$ ${ }^{1}$ Pediatric Allergy and Immunology, Gaziantep University, Gaziantep, Turkey; ${ }^{2}$ Pediatrics, Gaziantep University, Gaziantep, Turkey; ${ }^{3}$ Pediatric Allergy and Immunology, Hacettepe University, Ankara, Turkey}

Background: Even though some studies have investigated the genetic influences on corticosteroid response in chronic treatment of asthma, genetic associations of the response to single-high dose inhaled corticosteroid efficacy in acute asthma treatment is unknown.

Objective: To evaluate the role of genetic variants described a priori in the therapeutic response to single-high dose inhaled corticosteroids in children with moderate to severe asthma exacerbation.

Method: 82 children (56 boys/26 girls, mean age $9.6 \pm 3.2$ years) with moderatesevere asthma exacerbation were genotyped for the following SNPs: T-box 21 (TBX21) rs2240017 H33Q C $>$ G; glucocorticosteroid receptor (NR3C1) gene rs41423247 C>G, corticotrophin releasing hormone receptor 1 (CRHR1) gene rs1876828 $\mathrm{A}>\mathrm{G}$, rs242939 $\mathrm{A}>\mathrm{G}$ and rs242941 $\mathrm{G}>\mathrm{T}$. Children were treated with a single-high dose $(4000 \mu \mathrm{g})$ fluticasone propionate (FP) given by a nebulizer. Primary outcome measure was the improvement in FEV1 at $4 \mathrm{~h}$ of treatment. Results: Mean FEV1 was $71.7 \pm 14.2$ at presentation. Overall, FP treatment resulted in a significant improvement in asthma score $(P<0.0001)$ and FEV1 $(P<0.0001)$. Children with the CC genotype at NR3C1 rs41423247 $(n=26)$ had higher FEV1 improvement [24.2\% (IQR 11.5-36.3),) compared to those with $\mathrm{CG}+\mathrm{GG} \quad(n=19, \quad$ FEV $1 \% \quad$ improvement:7.9\% (6.1-24.6) $(P=0.006)$. Of the SNPs in the CRHR1, the genotype at rs242939 was associated with FEV1 improvement at $4 \mathrm{~h} ; 33.3 \%(31.6-41.7)$ in AG genotype $(n=5)$ vs $12.6 \%(7.1-26.2)$ in AA $(n=39)(P=0.016)$. SNPs at the TBX21 was not associated with FEV1 response at $4 \mathrm{~h}$.
Conclusion: SNPs at NR3C1 and CRHR1 may be associated with the response to single-high dose FP treatment in children with moderate to severe asthma exacerbation.

\section{3}

Genetic variation in NFKB1 is associated with allergic diseases and lung function in children

Soto-Ramirez, $N^{1} ;$ Karmaus, $W^{1} ; Z y i a b, A^{2} ;$ Zhang, $H^{1}$; Lockett, $\mathrm{G}^{3}$; Holloway, $\mathrm{J}^{3,4}$; Patil, $\mathrm{V}^{5,6}$; Arshad, $\mathrm{SH}^{5,6}$; Ewart, $\mathrm{S}^{7}$

${ }^{1}$ Epidemiology, Biostatistics \& Environmental Health University of Memphis, Memphis, TN, United States: ${ }^{2}$ Epidemiology and Biostatistics, University of South Carolina, Columbia, SC, United States; ${ }^{3}$ Human Development and Health, University of Southampton, Southampton, United Kingdom; ${ }^{4}$ Clinical and Experimental Sciences, University of Southampton, Southampton, United Kingdom; ${ }^{5}$ University of Southampton, Southampton, United Kingdom; ${ }^{6}$ David Hide Asthma and Allergy Research Centre, Isle of Wight, United Kingdom; ${ }^{7}$ Department of Large Animal Clinical Sciences, Michigan State University, East Lansing, MI, United States

Background: Allergic diseases are the most frequent chronic illnesses in children. Single nucleotide polymorphisms (SNPs) in the NFKB1 gene have been linked to asthma and respiratory diseases. No study has explored whether NFKB1 genotype is associated with immunoglobulin (Ig) E, eczema, allergic sensitisation (atopy), and lung function in adolescents. We aim to test whether NFKB1 SNPs are associated with asthma, eczema, atopy, serum IgE levels and lung function.

Method: In the 1989 Isle of Wight birth cohort $(n=1456)$ information on the prevalence of asthma and eczema was obtained at $1,2,4,10$, and 18 years of age. Skin prick tests (atopy) were carried out at ages 4, 10, and 18 years, total $\mathrm{IgE}$ and lung function measurements at 10 and 18 years. Seven tagging polymorphisms in the $N F K B 1$ gene were genotyped and, based on linkage disequilibrium, three SNPs were selected for further analyses. Log-linear models were employed to evaluate the relationship of SNPs with asthma, eczema, atopy, and serum $\operatorname{IgE}$ (dichotomized into $<200$ and $\geq 200 \mathrm{kU} / \mathrm{l}$ ), and linear regressions were used for the association of SNPs with lung function measurements adjusted for confounders.

Results: We detected significant associations with two SNPs within NFKB1: rs230498 was associated with elevated total serum $\mathrm{IgE}$ at age 10 (risk ratio (RR): 1.40; $P=0.02)$; and rs4648090 was linked to asthma risk at age 2 years (RR: 1.38, $P=0.02)$. Regarding lung function, the same two NFKB1 SNPs were associated with decreased forced vital capacity (FVC) and forced expiratory volume in one-second (FEV1) at 10 years of age. No associations were observed for eczema, atopy, and FEV1/FVC ratio.
Conclusion: This is the first study to demonstrate that SNPs within NFKB1 (rs230498 and rs4648090; both within introns) are associated with serum IgE, FVC and FEV1 in children.

\section{4 \\ Multiple loci associated with asthma in the Korean population}

Yoon, $\mathrm{D}^{1} ; \mathrm{Kim}, \mathrm{E}-\mathrm{J}^{1} ; \mathrm{Kim}, \mathrm{YJ}^{2}$; Chang, W-S ${ }^{1} ;$ Cho, S- $\mathrm{H}^{3}$; $\overline{\mathrm{Cho}, \mathrm{N}-\mathrm{H}^{4}}$; Kim, B-J $\mathrm{J}^{2}$; Han, B-G ${ }^{2}$; Park, C-S $\mathrm{S}^{5}$, Lee, J-S ${ }^{1}$ National Institute of Health, Center for Immunology and Pathology, Osong, Korea; ${ }^{2}$ National Institute of Health, Center for Genome Science, Osong, Korea; ${ }^{3}$ Department of Internal Medicine and Institute of Allergy, Seoul National University College of Medicine, Seoul, Korea; ${ }^{4}$ Department in Preventive Medicine, Ajou University School of Medicine, Suwon, Korea; ${ }^{5}$ Division of Allergy and Respiratory Medicine, Soonchunhyang University Bucheon Hospital, Bucheon, Korea; ${ }^{6}$ National Institute of Health, Osong, Korea

Background: Despite the previous efforts in finding asthma associated loci, the previously reported genetic loci explain only a small proportion of the heritability. Further analysis on unrevealed genetic component is required to solve the missing heritability problem. In this context, imputation based association analysis had gathered much attention to perform in silico fine mapping. In this study, we performed imputation based association study to identify multiple susceptibility loci for asthma using 1000 genomes project data.

Methods: The case-control samples consisted of 884 asthma (Illumina Human $660 \mathrm{~W}$ ) and 1143 controls (Affymetrix SNP array 5.0). The controls were selected from the Korean general population. The cases were recruited from the Asthma Genome Research Center, diagnosed by physician. Due to limited number of SNPs in overlap between platforms, we performed imputation on cases and controls using IMPUTE v2.3 software and 1000 genomes phase I interim as a reference panel. Statistical analysis was performed using PLINK and R statistics software. A logistic regression analysis was conducted adjusting for age, sex.

Results: We identified 12 novel asthma associated regions including the loci at 1p21, 2q14, 3p21, 6p22, 9q21, 11p15, $11 \mathrm{q} 14,12 \mathrm{q} 12,13 \mathrm{q} 13,18 \mathrm{q} 21,20 \mathrm{q} 13$, and 20 p12 $\left(P\right.$-value $\left.<1 \times 10^{-5}\right)$. Also, we replicated eight previously reported asthma susceptibility loci such as 4q31, HLADQA1 on chromosome 6, and SMAD3 on chromosome 15 ( $P$-value $<0.05)$.

Conclusion: In summary, we identified 12 new loci susceptible to asthma and replicated eight previously reported loci. To validate the genetic effect of discovered loci, the replication analysis in an independent cohort is required. 


\section{Oral Abstract Session}

\section{OAS 5 - Consequences of mast cell activation}

\section{5 \\ Tryptase measurements in acute anaphylaxis: specific cut-off points are needed depending on the age of the patients \\ Arroabarren, E; García, BE; Olaguibel, JM; Lizaso, MT; Echechipía, S; Tabar, Al \\ Complejo Hospitalario de Navarra, Servicio de Alergia, Pamplona, Spain}

Background: Many biological variables vary depending on the age of the patients. We seeked for differences in acute tryptase measurements in anaphylaxis according to the age of the patients.

Method: Retrospective review of 1505 tryptase determinations obtained in patients attending the Emergency Department of the Navarre Health System hospitals (2009-2012) for suspected allergy, including those meeting anaphylaxis criteria. Three age groups were considered: (A group) less than 15 years old, (B group) aged between 16 and 64, (C group) aged more than 65 . Rates of positive tryptase determinations $(>11.4 \mathrm{mcg} / \mathrm{l}$, as proposed by manufacturer) (Chi Square test), median of tryptase values during the episode and median duration of symptoms at sample obtention (Kruskall-Wallis test) were analysed. Optimal cut-off points for each group were calculated by a Receiver Operative Curve (ROC).

Results: We identified 376 anaphylaxis episodes (A group: 55 patients, B group: 270 patients and $\mathrm{C}$ group: 51 patients). Rates of positive tryptases: A: $32 \%$, B: $59.4 \%$, and $\mathrm{C}$ group: $78.8 \%(P<0.001)$. The median of tryptase determinations were: A group: $7.98 \mathrm{mcg} / 1$ (1-39.7 mcg/1), B: 11.25 $\mathrm{mcg} / \mathrm{l}(1.57-98.6 \mathrm{mcg} / \mathrm{l}), \mathrm{C}: 17 \mathrm{mcg} / \mathrm{l}$ (4.78$94.3 \mathrm{mcg} / \mathrm{l})(P<0.001)$. Median duration of symptoms: A: $1 \mathrm{~h}(0.25-24 \mathrm{~h}), \mathrm{B}: 1.5 \mathrm{~h}$ $(0.33-17 \mathrm{~h}), \quad \mathrm{C}$ group: $2 \mathrm{~h} \quad(0.1-6 \mathrm{~h})$ $(P>0.05)$. The optimal cut-off points established by ROC curve were: A: 7.19 $\mathrm{mcg} / \mathrm{l} ; \mathrm{B}: 7.56 \mathrm{mcg} / \mathrm{l}$, and $\mathrm{C}: 13.3 \mathrm{mcg} / \mathrm{l}$. (Areas under the curve: $0.735,0.77$ and 0.815 , respectively).

Conclusion: The rate and median of tryptase during anaphylaxis increase with the age of the patients. Specific cut-off points in acute tryptase determinations are needed to accurately assess patients of different ages.
26

Old-age. More than a risk factor in anaphylaxis

Vela, C; Arroabarren, E; Garcia, BE; Rodriguez, A; Garrido, S; Alvarez-Puebla, MJ

Complejo Hospitalario de Navarra, Servicio de Alergia, Pamplona, Spain

Background: Old age is a risk factor (RF) for severe anaphylaxis. Information regarding specific features in these patients remains incomplete.

Method: Retrospective review of 1349 total tryptase determinations obtained in adults attending the Emergency Department of the Navarre Health System hospitals (2009-2012) due to suspected allergy, including those meeting anaphylaxis criteria. We seeked for differences between patients aged less than 65 (A) and more than 65 years old (OA) in: symptoms, anaphylaxis severity, eliciting agent (EA), RF and tryptase positive rates $(>11.4 \mathrm{mcg} / 1$ proposed by manufacturer) (Chi Square test). Results: We identified 311 anaphylactic episodes (A group: 260 and OA group: 51 cases).

Symptom distribution (A group): skin: $98.8 \%$, respiratory: $76.2 \%$, digestive: $39.6 \%$, cardiovascular: $38.1 \%$ and neurological $28.1 \%$. Symptom distribution (OA group): skin $(100 \%)$, respiratory $(63.5 \%)$, cardiovascular $(40.4 \%)$, digestive $(38.5 \%)$ and neurological symptoms $(26.9 \%)$ $(P>0.05)$.

Assessment of severity (A group): mild $(24.8 \%)$, moderate $(38.3 \%)$, severe $(37 \%)$ cases. Severity in OA group: mild $(11.5 \%)$, moderate $(32.7 \%)$, severe episodes $(55.8 \%)$ $(P=0.007)$.

$\mathrm{RF}$ in A group: Non-existent: $61.5 \%$ patients, previous anaphylaxis: $14.7 \%$ cases, cardiovascular disease $11.8 \%$, asthma $11.2 \%$, mastocitosis: $0.8 \%$. OA group risk factors: Non-existent: $31.4 \%$, cardiovascular disease: $48 \%$, asthma: $7.6 \%$ and $8 \%$ previous anaphylaxis $(P<0.001)$.

EA in A group were: drugs $43.8 \%$, foods $37.3 \%$, Anisakis: $5.3 \%$, Hymenoptera: $3.8 \%$, and non-identified in $9.8 \%$. EA in OA group: drugs: $55.8 \%$, foods: $17.3 \%$, Anisakis $11.5 \%$, Hymenoptera: $5.8 \%$, 9.5\% non-identified $(P=0.056)$.

Tryptase determinations were positive in $49.6 \%$ of $\mathrm{A}$ and in $78.8 \%$ of OA patients $(P=0.001)$.
Conclusion: Anaphylaxis at older age has not only different clinical features (severity and co-existing RF) but analytical ones (more frequent tryptase rises).

\section{7}

KIT D816V mutation analysis in blood as a screening tool for mastocytosis

Broesby-Olsen, $\mathrm{S}^{1}$; Bindslev-Jensen, $\mathrm{C}^{1}$; Vestergaard,

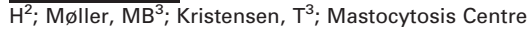
Odense University Hospital (MastOUH) ${ }^{1}$ Department of Dermatology and Allergy Centre, Odense Research Centre for Anaphylaxis (ORCA), Odense University Hospital, Odense, Denmark; ${ }^{2}$ Department of Hematology, Odense University Hospital, Odense, Denmark; ${ }^{3}$ Department of Pathology, Odense University Hospital, Odense, Denmark

Background: Mastocytosis is considered rare, however probably underdiagnosed. Current clinical tools for recognizing mastocytosis comprise constellation of clinical symptoms, presence of mastocytosis skin lesions and baseline s-tryptase. It is well known that symptoms may be very heterogeneous, that skin lesions may be absent or inconspicuous and that tryptase is not always elevated in mastocytosis. Development of highly sensitive methods for KIT D816V mutation detection suggests that analysis of peripheral blood (PB) may represent a promising screening test for mastocytosis. However, a systematic assessment of the sensitivity and specificity of this approach is needed in order to establish its value in clinical use.

Methods: In a retrospective register study we evaluated sensitive KIT D816V mutation analysis of PB as a diagnostic test in an entire hospital based case-series of adults with mastocytosis.

Results: The mutation was detected in PB in $91 / 97$ (94\%) patients - including 78/83 (94\%) systemic mastocytosis (SM) and $3 / 4$ $(75 \%)$ cutaneous mastocytosis (CM) patients. The test was $100 \%$ specific as determined by analysis of 20 healthy blood donors and 165 clinically relevant control patients who all tested negative. Importantly, the control group included 24 patients in whom a full bone marrow (BM) investigation evaluating WHO SM criteria, including sensitive flow cytometry of BM aspirate had been performed. Mutation analysis of PB was significantly more sensi- 
tive than tryptase $>20 \mathrm{ng} / \mathrm{ml}$ that were only present in 50/77 (65\%) of SM-patients and none of CM-patients. Of 27 SM-patients with low tryptase, $26(96 \%)$ tested mutation positive.

Conclusion: In this study we demonstrate for the first time that by using a highly sensitive method it is possible to detect the KIT D $816 \mathrm{~V}$ mutation in $\mathrm{PB}$ in nearly all adult mastocytosis patients. The test is readily available and the present results provide evidence to support the inclusion of this approach in diagnostic algorithms and as a screening tool for mastocytosis.

\section{8 \\ The replication of whole genome expression profile in patients with mastocytosis using $\mathbf{1 6}$ genes RT-PCR results \\ Niedoszytko, $\mathrm{M}^{1}$; Gorska, $\mathrm{A}^{1}$; Skrzypski, $\mathrm{M}^{2}$; Maciejewska, $A^{3}$; Chelminska, $M^{1}$; Pawlowski, $R^{3}$; Jassem, $E^{1}$ \\ ${ }^{1}$ Allergology, Medical University of Gdansk, Gdansk, Poland; ${ }^{2}$ Oncology, Medical University of Gdansk, Gdansk, Poland; ${ }^{3}$ Forensic Medicine, Medical University of Gdansk, Gdansk, Poland}

Introduction: The epidemiological data indicate that the incidence of anaphylaxis is increasing worldwide. Mastocytosis may be a risk factor for mastocytosis in some patients. The serum concentration of tryptase may be used as a marker of mastocytosis, however in some patients the disease may be diagnosed in spite of low tryptase level. Our previous results has shown the difference in expression of genes in peripheral blood of mastocytosis patients. The current study aimed to replicate these results using RT-PCR method in Polish population of mastocytosis patients.

Methods: The group of 59 mastocytosis patients (including $24 \mathrm{CM}, 33$ ISM and two SSM subjects) and 20 controls was studied. Based on the results of previous studies a set of 16 genes was selected in the study including (18S-Hs99999901_s1, ABI3BP-Hs00227206_m1,ANKRD6-

Hs00208902_m1,B3GAT1 Hs00218629_m1, CDC42BPA-Hs00177522_m1, DV̄L1Hs00182896_m1, G0S2-Hs00274783_s1, HLA-DRB4-Hs03027795 uH, ITGĀVHs00233808_m1, ITGB1-Hs00559595_m1, JUP-Hs00158408_m1, MAP2K3Hs00177127_m1, MLL3-Hs01005521_m1, TNFRSF4-Ḧs00533968 m1, TRĀF4Hs00188755_m1, ZMAT3-Hs00536976_m1.

RT PCR method (Applied Biosystems, Foster, CA, United States) was used to analyze the difference in expression of selected genes in peripheral blood.

Results: The significant difference in expression of B3GAT1 $(P=0.003)$ and ITGB1 $(P=0.01)$ were found between mastocytosis and controls. The difference in expression of B3GAT1was found in SSM $(P=0.008)$ ISM $(P=0.012)$ and CM $(P=0.016)$ patients. The difference in ITGB1 gene expression was present only in systemic mastocytosis patients. B3GAT1 is a member of the glucuronyltransferase gene family. This gene product functions as the key enzyme in a glucuronyl transfer reaction during the biosynthesis of the carbohydrate epitope HNK-1, known also as CD57. It acts to add a glucuronic acid (GlcA) to the terminal $\mathrm{N}$-acetyllactosamine (Lac) disaccharide to form the HNK-1 epitope precursor. ITGB1 product is involved in cancerogenesis i.e. in occurrence and metastasis of the lung cancer.

Conclusion: The current study show that the expression of two out of 16 studied genes was different in mastocytosis patients. Further studies are necessary to confirm these results as a step to construct a clinical method.

\section{9}

Unaltered mast cell reactivity in patients with mast cell activation disorders

Gulen, $T^{1,2,3}$; Möller Westerberg, $C^{2}$; Kolmert, $\mathrm{J}^{2}$; Larsson, $J^{3}$; Dahlén, S-E $E^{3}$; Dahlén, $\mathrm{B}^{2,3}$; Nilsson, $\mathrm{G}^{2,3}$ ${ }^{1}$ Department of Respiratory Medicine and Allergy, Karolinska University Hospital Huddinge, Stockholm, Sweden; ${ }^{2}$ Karolinska University Hospital and Karolinska Institutet, Mastocytosis Center Karolinska, Stockholm, Sweden; ${ }^{3}$ Karolinska Institutet, Centre for Allergy Research, Stockholm, Sweden

Background: Mast cell activation disorders (MCAD) comprise a heterogeneous spectrum of disorders characterised by unregulated increased activation of mast cells (MC) leading to pathologically enhanced release of mediators. Patients presented with recurrent signs of MC mediatorrelated symptoms differ in the severity. In this study, we sought to determine baseline MC reactivity of cutaneous and bronchial MCs in subjects with different MCAD phenotypes and compare them with normal control subjects.

Method: A total of 31 consecutive patients were enrolled to this study including 22 patients with suspected MCAD, and 9 healthy controls. All subjects underwent an allergy work-up and we measured skin sensitivity to histamine and MC degranulator morphine via skin prick test. In addition, we determined serum total IgE levels, and baseline serum tryptase (sBT) and urinary metabolites of histamine and prostaglandin $\mathrm{D}_{2}$. We also performed mannitol airway challange and measured exhaled nitric oxide (FENO) levels. Moreover, all 22 MCAD-prone subjects underwent a bone marrow investigation. We evaluated clonal markers of bone marrow MCs by morphology, flow cytometry and detection of KIT D816V mutation.

Results: Using current WHO diagnosticcriteria, 15 MCAD patients were diagnosed with systemic mastocytosis (SM); four with monoclonal mast cell activation disorder and remaining three had diagnosis of nonclonal idiopathic anaphylaxis. The skin reactivity to histamine or morphine was not significantly different between the groups. There were no differences in total serum IgE levels either. In addition, exhaled nitric oxide levels and airway reactivity to mannitol were indifferent between the groups. Notably, levels of all three MC mediators, tryptase and metabolities of histamine and $\mathrm{PGD}_{2}$, were significantly higher in SM patients compared to other subjects with MCAD and normal controls.

Conclusion: In this study, we detected significantly increased levels of baseline MCmediators indicating sign of hyperreactivity in MC of SM patients. Remarkably, we did not observe any increased reactivity in the skin MC measured by sensitivity to histamine or morphine, nor in the airway $\mathrm{MC}$ of MCAD patients assessed by mannitol chalange. Alterations in the MC-reactivity in MCAD patients seem therefore to be complex and underlying mechanisms remain to be elusive.

\section{0}

Level of serum IgE and serum tryptase in mastocytosis: a retrospective analysis in Greek population

Gkavogiannakis, N; Potika, M; Makris, M; Chatzipetrou, A Allergy Unit 'D. Kalogeromitros', 2nd Department of Dermatology and Venereology, National University of Athens Medical School, University General Hospital 'Attikon', Mastocytosis Outpatient Clinic, Athens, Greece

Background: The role of IgE in mastocytosis remains obscure. We hypothesize that due to the increased number of mast cells IgE must be captured on the FcReI thus, reducing the concentration of serum circulating IgE.

To this end, we examined the serum concentration of IgE in correlation with serum tryptase and disease pattern in a wellcharacterised sample of mastocytosis individuals.

Method: Sixty-two pts (36 females; mean age $38 \pm 16$ years) attending the Mastocytosis Outpatient Clinic were retrospectively analyzed. Mastocytosis was assessed according to WHO criteria; $30.6 \%$ had skin mastocytosis, $37.1 \%$ had systemic mastocytosis, $1.6 \%$ had isolated mastocytoma while $30.6 \%$ had increased serum tryptase and suggestive clinical manifestations but had not undergone bone marrow 
biopsy. Among all of them, 12/62 participants had positive the mutation of c-kit D816V in mast cells while half of them 6/ 12 had the mutation in all blood cell series (a subgroup of ISM with potentially more severe evolution). Statistical analysis was performed using SPSS 17.0 software.

Results: In individuals suffering from skin mastocytosis serum $\mathrm{IgE}$ was $99 \pm 100 \mathrm{IU} /$ $\mathrm{ml}$ and serum tryptase $11 \pm 12 \mu \mathrm{g} / \mathrm{ml}$ respectively. Systemic mastocytosis patients had serum $\operatorname{IgE} 38 \pm 48 \mathrm{IU} / \mathrm{ml}$ and serum tryptase $84 \pm 91 \mu \mathrm{g} / \mathrm{ml}$. Statistical analyses has shown a significant difference in one parameter (serum tryptase skin vs systemic mastocytosis $P=0.003$, serum IgE skin vs systemic mastocytosis $P=0.089$ ). Moreover, the subgroup of all blood cell series mutation carriers had mean of serum $\operatorname{IgE}$ $38 \pm 56 \mathrm{IU} / \mathrm{ml}(50 \%$ below $16 \mathrm{IU} / \mathrm{ml})$ and mean of serum tryptase $105 \pm 70 \mu \mathrm{g} / \mathrm{ml}$ (83.3\% above $70.1 \mu \mathrm{g} / \mathrm{ml})$.

Conclusion: In our population patients suffering from mastocytosis have relatively low values of $\operatorname{IgE}$ while this seems more prominent for systemic mastocytosis compared with sole skin disease. Further studies are needed to confirm this interesting hypothesis. 


\section{Oral Abstract Session}

\section{OAS 6 - Pollen: new foes}

\begin{abstract}
31
Family-based analysis of atopic sensitisation in immigrant families with small children in Malmö, Sweden

Richter, JC ${ }^{1}$; Jakobsson, $\mathrm{K}^{2}$; Taj, TM

Division of Occupational and Environmental Medicine, Faculty of Medicine, Skåne University Hospital, Lund University, Lund, Sweden; ${ }^{2}$ Division of Occupational and Environmental Medicine, Lund University, Skåne

University Hospital, Lund, Sweden
\end{abstract}

Background: It is well known that atopic sensitisation and allergic diseases in immigrant populations are influenced by a multitude of factors related to both the host society and to adopted lifestyles. However, studies with family-based investigations approaches are scarce.

Methods: The data was collected as part of an ongoing study into the health of children living in a disadvantaged immigrant neighbourhood in Malmö/Sweden, where a large number of structurally unsound apartments existed. Data were collected on apartment characteristics, lifestyles, and behaviours as well as demographic information on all family members in the households. The sample was enriched with regard to respiratory diseases by identifying families of children who had had pertinent contacts with the primary health care system. Skin prick tests (SPT) with a Swedish standard panel of ten aeroallergens, and additionally cockroach allergens, were performed in children and parents.

Results: Overall the study included 668 participants, 358 of which were children between 0 and 13 years. In total 424 SPT were available. Single or multiple sensitisations to common aeroallergens was observed in $20 \%$ of the tests, in decreasing order to pollen, mites and furry pets. Test from children and at least one parent was available for 73 families. Family-based data analyses are under way.

Conclusion: Atopic sensitisation to allergens from the host country was frequent not only in children of immigrant families, but also in adults, who arrived in the host society during adulthood. Clinicians involved in the care of these populations should have a low threshold for targeted investigations when warranted by the clinical presentation.
32

Health impact of pollen exposure

Thibaudon, $\mathrm{M}^{1}$; Oliver, $\mathrm{G}^{1}$; Berger, $\mathrm{U}^{2}$; Bastl, $\mathrm{K}^{2}$ 'RNSA, Brussieu, France; ${ }^{2}$ Medical University of Vienna, Vienna, Austria

Background: In France, a fraction of 10 to $20 \%$ suffers from pollen allergies. The French network of air monitoring (RNSA) analyzes the biological content of the air and is responsible for informing the French population about allergy risks. In this study, the aim is to establish a relation between the pollen concentration and the symptom severity and to determine the threshold of sensibility of individual users. Method: For this purpose information on the user's location (biogeographical regions), meteorological parameters and phenological data has been taken into account. Pollen types chosen for this study belong to the most allergenic taxa like ragweed, birch, cypress and grasses. Pollen exposure measurements are based on data from Hirst-type spore traps and correspond to the daily pollen concentration in a region. Health impact was assessed based on the entries of Patient's Hayfever Diary users, who fill in their overall feeling, organ specific symptoms and medication use on a web-based platform. All data was included to calculate a symptom score.

Results: The results show that there is a dose-response relationship between the pollen exposure and the symptom severity for all the pollen types included in this study. Thresholds for onset of symptoms vary between different allergenic pollen types. Moreover, thresholds vary annually even for the same taxon. Biogeographical variation in symptom severity was also observed.

It is suggested here that the assessed variation could by caused by climatic factors and the presence of atmospheric pollutants that could influence both the production and dispersal of pollen and the sensitivity of pollen allergy sufferers.

Conclusion: Concluding, this study gives insight into the relation between health condition and pollen exposure of pollen allergy sufferers. Participation of many institutions and decision makers among them the RNSA, health authorities, medical institutions and botanical and ecological research communities is needed to cover a major topic like pollen allergy.

33

Aerobiological survey of ragweed (Ambrosia artemisiifolia) expansion in the Geneva area: a tool for the control of the plant

Gumowski, $\mathrm{PI}^{1}$; Graber, $\mathrm{M}-\mathrm{J}^{2}$; Van Eck, $\mathrm{F}^{1}$; Echenard, $\mathrm{M}^{1}$; Clot, $\mathrm{B}^{2}$

IINRAAIC Hôpital de la Tour, Immunology Allergology, Meyrin, Switzerland; ${ }^{2}$ MeteoSwiss, Payerne, Switzerland

Background: Prolonged and repeated periods of exposure are major factors of ragweed pollen sensitivity and reactivity. Fighting against the expansion of ragweed since its appearance in the Geneva area in 1998 has been a major concern of sanitary, agronomical and environmental authorities; priority goals were to stop the expansion of ragweed, try to eradicate the plants and prevent its propagation further inside Switzerland. Intensive plant treatments from 2000 to 2008 have allowed the control but not a complete eradication of ragweed colonisation in the Geneva area. Regular field inspections and an aerobiological survey have since been maintained as control of a possible resurgence of ragweed.

Methods: Daily average pollen concentrations [RG]were measured with two Burkard pollen and spores traps. Locations: [HUG] University Hospital Geneva (altitude of $380 \mathrm{~m}$ and about $40 \mathrm{~m}$ above ground level), [HDT] Hôpital de la Tour in Meyrin (altitude of $435 \mathrm{~m}, 20 \mathrm{~m}$ above ground level).

Results: Between 2009 and 2011 collected datas from HUG and HDT showed good convergence: reflecting mostly RG originating from the south east of France. In 2012, HDT detected RG 7 more days than HUG, outside the period of distant RG flights. In 2013, HDT detected RG for a total of 35 days, of which 22 consecutive days, while HUG recorded only 20 days with RG. $2 \mathrm{~h}$ analysis confirmed local emission sources. On site investigations demonstrated extensive colonisations on various sites recently used by constructions enterprises around Meyrin. 
Conclusions: Those observations stress again the importance of maintaining regular surveys of ambrosia dispersion once an area has been colonised, but also the role of human activities in propagating the plant. The incidence on new allergic sensitisation in the Meyrin area following this long exposition period will have to be assessed. Aerobiological survey will also enable to evaluate the efficacy of the treatment undertaken on the sites colonised by ragweed.

34

Pollen concentrations and emergency department visits for asthma and wheeze in Lisbon

Belo, $\mathrm{J}^{1}$; Carreiro-Martins, $\mathrm{P}^{1,2,3}$; Papoila, $\mathrm{AL}^{4,5}$

Geraldes, $\mathrm{C}^{6}$; Paiva, $\mathrm{M}^{1}$; Caeiro, $\mathrm{E}^{7}$; Ferro, $\mathrm{R}^{7}$; Coelho, $\mathrm{M}^{8}$; Leiria-Pinto, $\mathrm{P}^{1}$

${ }^{1}$ Allergy Department, Hospital de Dona Estefânia, Centro Hospitalar de Lisboa Central, EPE, Lisbon, Portugal; ${ }^{2}$ CEDOC, Faculdade de Ciências Médicas da Universidade Nova de Lisboa, Lisbon, Portugal; ${ }^{3}$ Centro Hospitalar de Lisboa Central, EPE, Hospital de Dona Estefânia, Gabinete de Apoio Estatístico e

Epidemiológico, Lisbon, Portugal: ${ }^{4}$ Faculdade de Ciências Médicas da Universidade Nova de Lisboa, Informatics and Biostatistics, CEAUL, Lisbon, Portugal; ${ }^{5}$ Centro Hospitalar de Lisboa Central, EPE, Gabinete de Apoio Estatístico e Epidemiológico, Lisbon, Portugal;

${ }^{6}$ Faculdade de Ciências Médicas da Universidade Nova de Lisboa, Informatics and Biostatistics, Lisbon, Portugal; ${ }^{7}$ Sociedade Portuguesa de Alergologia e Imunologia Clinica, Évora, Portugal; ${ }^{8}$ Pediatric Department, Hospital de Dona Estefânia, Centro Hospitalar de Lisboa Central, EPE, Lisbon, Portugal

Background: Previous studies reported associations between aeroallergen exposure and asthma exacerbations. However, the specific pollens implicated have not been consistent across studies, which may be partially attributable to geographic differences. The aim of our study was to investigate the short-term effects of airborne pollen counts on emergency department (ED) visits for asthma and wheeze in Lisbon area, from March to September, in the years 2002, 2004, 2006 and 2008, for children aged 6-17 years.

Methods: Counts were available for common pollens (Betulaceae, Cupressaceae, Parietaria, Pinus, Poaceae, Quercus, Olea, Chenopodium, Plantago and Compositae). Daily data on ED visits for asthma attacks and wheeze were obtained from Hospital Dona Estefânia (the main Pediatric Hospital in Lisbon) by a systematic random sampling. Short-term effects of pollen counts were assessed using distributed nonlinear lag models in order to study the relationship between pollen counts and respiratory events at different times after the exposure.

Results: Daily data from 856 days were analyzed. After adjustment for metereolog- ical variables and day of the week, a shortterm association was observed between Parietaria, Pinus and Cupressaceae pollen counts and ED visits for asthma and wheeze. These associations tended to be of the highest magnitude for Parietaria $(\mathrm{RR}=$ $1.06 ; 1.01-1.11 ; \quad P=0.015)$ and Pinus $(\mathrm{RR}=1.08 ; 1.01-1.16 ; P=0.022)$ on the third following day and for Cupressaceae $(\mathrm{RR}=1.03 ; 1.01-1.05 ; P=0.001)$ on the first following day (RR calculated for 10 grains $/ \mathrm{m}^{3}$ increments).

Conclusion: Parietaria, Pinus and Cupressaceae pollens presented a time lag effect on ED visits for asthma and wheeze in Lisbon. These findings need to be confirmed by future studies that consider also respiratory infections.

\section{5}

\section{Isolated increase in pollen sensitisation} over 20 years among Swedish adults

Bjerg, $A^{1}$; Eriksson, $J^{1}$; Ekerljung, $L^{1}$; Wennergren, $G^{2}$; Lötvall, $\mathrm{J}^{1}$; Lundbäck, $\mathrm{B}^{1}$

${ }^{1}$ Department of Internal Medicine and Clinical Nutrition, Krefting Research Centre, Sahlgrenska Academy,

University of Gothenburg, Göteborg, Sweden;

${ }^{2}$ Department of Paediatrics, University of Gothenburg, Gothenburg, Sweden

Background: Only few studies have assessed time trends in aeroallergen sensitisation in the general adult population. We studied allergic sensitisation in a general population of adults, and trends in sensitisation over two decades.

Method: Out of 18072 participants in the West Sweden Asthma Study, a random sample of 1472 aged 16-60 years were invited to skin prick testing (SPT) against 11 common aeroallergens during 2009-12. Complete SPTs were performed in 788 subjects $(57.3 \%$ women). The 303 subjects aged 20-46 and living in Gothenburg were compared to a similar sample from ECRHS in $1991-2(n=589)$.

Results: Sensitisation was seen in $42.0 \%$, and $13.7 \%$ were sensitised to four or more allergens. The dominating allergens were timothy $(24.7 \%)$, birch $(21.2 \%)$, cat $(17.9 \%)$ and $\operatorname{dog}(14.9 \%)$. Any mite sensitisation was seen in $12.6 \%$ while only $2.0 \%$ were sensitised to molds. In Gothenburg sensitisation to pollen had increased significantly since 1991-2: timothy $17.1 \%-$ $27.7 \%(P<0.001)$ and birch $15.6 \%-21.8 \%$ $(P=0.021)$, while the prevalence of all other studied allergens were unchanged. Male sex and a family history of rhinitis were associated with sensitisation, adjusted OR 1.6-2.5 and OR 1.8-2.8 depending on the studied allergen group. The risk was lower in subjects who had grown up with horses/cows, adjusted OR 0.5 (0.3-0.995) or cats/dogs, OR 0.7 (05-0.98), and in current smokers, OR $0.5(0.3-0.8)$. The risk of sensitisation with concomitant wheeze or rhinoconjunctivitis was also significantly decreased in subjects who had grown up with animals.

Conclusion: In this general sample of Swedish adults sensitisation to aeroallergens was common. The isolated increase in sensitisation to pollens points toward allergen-specific causes such as air pollution, which is known to specifically increase the allergenicity of pollens. Factors related to farm exposure, especially keeping of cows/ horses, but also keeping of furred pets, decreased the risk of sensitisation and allergic symptoms.

36

New developments for pollen allergy sufferers: personal pollen information and the symptom load index

Berger, UE ${ }^{1}$; Kmenta, $\mathrm{M}^{1}$; Jaeger, $\mathrm{S}^{1}$; Bergmann, $\mathrm{K}-\mathrm{C}^{2}$; Thibaudon, $\mathrm{M}^{3}$; Bastl, $\mathrm{K}^{1}$

${ }^{1}$ Department of Oto-Rhino-Laryngology, Medical University Vienna, Vienna, Austria; ${ }^{2}$ Charité Berlin Allergy-Centre-Charité, Klinik für Dermatologie, Venerologie und Allergologie, Berlin, Germany; ${ }^{3}$ RNSA, Brussieu, France

Background: Pollen information is a key factor to allergen avoidance and essential for an increasing part of the European population affected by pollen allergies. Hitherto, this information was not personalised, although there is a strong need for individual information because allergen content, pollen loads, and pollen allergy symptoms vary per region and year. Allergic reactions seem to vary also intra-individually.

Method: Only personal symptoms are needed in combination with information used before such as pollen data, meteorological data, aerobiological data and forecast model data to offer pollen allergy sufferers personalised pollen information. Users are classified based on their status from the last five days and receive a modified forecast for the next three days.

Results: Calculations are made each day, so that changes in development of symptoms can be rapidly tracked. An additional tool is the symptom load index - a measurement for the severity of a pollen season. This index gives a number for a single person, a defined population of a specific area during a defined time interval (day, season or year). The symptom load index allows an evaluation of e.g. pollen seasons in different years apart from pollen data.

Conclusion: This new measurement bears the potential for further improvements of pollen information. 


\section{Oral Abstract Session}

\section{OAS 7 - New insights in immunological mechanisms in food allergy}

37

Apical exposure to dietary non-digestible oligosaccharides and bacterial CpG DNA suppresses Th2 type chemokine release by activated intestinal epithelial cells

Overbeek, $\mathrm{SA}^{1}$; de Jager, $\mathrm{W}^{2}$; Garssen, $\mathrm{J}^{1,3}$; Willemsen, LEM $^{1}$

${ }^{1}$ Division of Pharmacology, Utrecht Institute for Pharmaceutical Sciences, Utrecht University, Utrecht, The Netherlands; ${ }^{2}$ University Medical Centre Utrecht, Pediatric Immunology, Laboratory for Translational Immunology, Utrecht, The Netherlands; ${ }^{3}$ Nutricia

Research, Immunology, Utrecht, The Netherlands

Background: Intestinal epithelial cells (IEC) instruct dendritic cells (DC). We previously showed dietary short chain galactoand long chain fructo-oligosaccharides (scGOS/lcFOS) combined with TLR9 ligand $\mathrm{CpG}$ DNA to affect epithelial function. Epithelial derived IL- $1 \alpha$ contributes to allergic sensitisation to inhaled house dust mite via induction of IL-33 secretion by IEC (Willart et al. 2012). Beyond the secretion of cytokines IEC produce a broad range of chemokines capable of instructing DC. The effect of IL- $1 \alpha$ on Th2 polarizing chemokine release by activated IEC and the modulatory effect of scGOS/ lcFOS and $\mathrm{CpG}$ was studied.

Methods: HT-29 cells (human colon adenocarcinoma cell line; IEC) were cultured on transwell filters. After reaching confluency, the cells were pre-incubated basolaterally with IL- $1 \alpha \pm \operatorname{IFN} \gamma$ and TNF $\alpha$ and apically with scGOS/lcFOS $\pm \mathrm{CpG}$ for $6 \mathrm{~h}$. Subsequently, the cells were washed and medium was added basolaterally, whereas the cells were apically exposed to the dietary components for $24 \mathrm{~h}$. Production of Th2 polarizing chemokines CCL2, CCL5, CCL20 and CCL22 and galectin-9 and TGF- $\beta$ was measured.

Results: CCL20 was significantly increased after IL- $1 \alpha$ activation, which enhanced further in the presence of IFN $\gamma+\mathrm{TNF} \alpha$. $\mathrm{IFN} \gamma+\mathrm{TNF} \alpha$ activation significantly induced the release of CCL2, CCL5 and CCL22 by the HT-29 cells, which was more prominent in the presence of IL- $1 \alpha$. Moreover, IFN $\gamma+\mathrm{TNF} \alpha \pm$ IL- $1 \alpha$ activation significantly led to an increase of galectin- 9 and TGF- $\beta$ release. Intervention with the dietary components scGOS/lcFOS $\pm \mathrm{CpG}$ reduced the release of CCL2,
CCL5, CCL20 and CCL22, while the levels of galectin- 9 and TGF- $\beta$ remained high. Conclusion: IL- $1 \alpha$ boosts the release of $\mathrm{Th} 2$ polarizing chemokines by IFN $\gamma$ and TNF $\alpha$ activated IEC and vice versa. Dietary scGOS/lcFOS and $\mathrm{CpG}$ suppresses this response, skewing away from the allergic phenotype.

This study was performed within the framework of Dutch Top Institute Pharma (project \# T1.501).

38

Transport of Pru p 3 across gastrointestinal epithelium - an essential step towards the induction of food allergy?

Gómez-Casado, $\mathrm{C}^{1}$; Tordesillas, $\mathrm{L}^{1}$; Garrido-Arandia, $\mathrm{M}^{1}$; Murua-García, $A^{1}$; Palacín, $A^{1}$; Varela, $J^{2}$; Konieczna, $P^{3}$; Cuesta-Herranz, J4 ; Akdis, $\mathrm{CA}^{3}$; O'Mahony, $\mathrm{L}^{3}$; Díaz-Perales, $A^{1}$

${ }^{1}$ CBGP/Technical University of Madrid, Pozuelo de Alarcón, Spain; ${ }^{2}$ Service of Protein Chemistry, Centre for Biological Research, CSIC, Madrid, Spain; ${ }^{3}$ Swiss Institute of Allergy and Asthma Research (SIAF), University of Zurich, Davos, Switzerland; ${ }^{4}$ Allergy Service, Jimenez Diaz Foundation, Madrid, Spain

Background: Since intestinal absorption of food proteins can trigger an allergic reaction, we aimed to characterise the impact of Pru p 3, peach major allergen, on mucosal epithelial immune response. For that, the effect of Pru p 3 on intestinal epithelial cell permeability and its ability to cross the epithelial cell monolayer were evaluated.

Method: Enterocytic monolayers were established by culturing Caco-2 cells on permeable supports that separate the apical and basolateral compartments. Pru p 3 was added to the apical chamber, and the protein transported to the basolateral chamber was quantified by ELISA with specific antibodies. Transepithelial electrical resistance (TEER) of the cell culture was measured along the experiment to check monolayer integrity. In addition, cytokines produced by epithelial cells in response to the allergen were quantified.

Results: Pru p 3 was able to cross the monolayer without disturbing the integrity of the tight junctions. The peach allergen that crossed the cell monolayer was detected and quantified in the cell fraction and in the basal medium compartment by immunodetection with specific antibodies. This transport was significantly higher than that of a non-allergenic peach LTP, LTP1, and occurred via a receptor-mediated pathway. The incubation of Caco-2 cells with Pru p 3 triggered the expression of epithelial-specific cytokines, TSLP, IL33 and IL25.

Conclusion: These results suggest that Pru p 3 was able to cross the cell monolayer by the transcellular route and induce the production of Th2 cytokines. The results of the present study represent a step towards clarifying the importance of Pru p 3 as a sensitiser.

39

The relevance of intracellular allergen degradation inside antigen presenting cells for food allergy

Smit, JJ; van Roest, M; Pieters, RHH Immunotoxicology, Institute for Risk Assessment Sciences, Utrecht University, Utrecht, The Netherlands

Background: Digestive degradation of proteins has been shown to determine allergenicity. Most allergens are usually stable to gastrointestinal digestion, or proteins become allergenic when digestion is inhibited. However, it is unclear how allergens are degraded inside pivotal immune cells such as dendritic cells (DC), and how this affects the subsequent immune response to these allergens.

Methods: In our studies, we determined whether (i) we are able to measure uptake and degradation of allergens inside DC and (ii) differences in above mention factors exist between allergens, including the peanut allergens Ara h1, 2, 3 and 6 .

Results: Using bone marrow-derived DC and fluorescent-labeled proteins, we observed that cellular uptake of Ara h1 was much higher than Ara h2, 3 and 6 . Moreover, using proteins coupled to beads we observed that intracellular protein degradation was higher for Ara h1 and Ara h3 than for Ara h2 and 6. Finally, when Ara $\mathrm{h} 1,2,3$ or 6 specific CD $4+\mathrm{T}$ cells were added to matching allergen-pulsed 
DC, we observed that while Ara h1, 3 and to lesser extend Ara h6 elicited strong Th2 type responses, Ara h2 did not elicit any $\mathrm{T}$ cell responses. This suggest that a lower level of allergen degradation in DC leads to lower or defective $\mathrm{T}$ cell responses. Interestingly, further studies showed that extracellular danger signals, including endotoxin and cholera toxin, inhibited the uptake and degradation of allergens.

Conclusion: We showed that allergenicity of (peanut) proteins may be determined at the level of allergen breakdown inside the antigen presenting cell. These findings may be relevant to the risk of existing allergens but also to the risk of novel or modified proteins. This also illustrates the usefulness of in vitro, cell based assays to examine initial responses to potential allergens at the beginning of the allergic cascade.

\section{0}

Intracellular IL-1 $\alpha$ modulates peanut-induced anaphylaxis in a model of skin sensitisation

Jimenez-Saiz, R${ }^{1}$; Mandur, $\mathrm{TS}^{1}$; Kong, $\mathrm{J}^{1}$; Flader, $\mathrm{K}^{1}$; Gordon, $\mathrm{ME}^{1}$; Walker, $\mathrm{T}^{1}$; Goncharova, $\mathrm{S}^{1}$; Chu, $\mathrm{DK}^{1}$; Stämpfli, $M^{1}$; Richards, $C^{1}$; Waserman, $S^{1}$; Humbles, $\mathrm{AA}^{2}$; Kolbeck, $\mathrm{R}^{2}$; Jordana, $\mathrm{M}^{1}$

${ }^{1}$ McMaster Immunology Research Centre (MIRC), McMaster University, Hamilton, ON, Canada;

${ }^{2}$ Medlmmune LLC, Gaithersburg, MD, United States

Background: Increasing evidence indicates that the skin can be an initiating site for peanut (PN) allergy. Our laboratory has established a model of epicutaneous sensitisation and anaphylaxis to PN aimed at dissecting the cellular and molecular basis regulating anaphylaxis in order to uncover novel therapeutic targets.

Method: The model relies on the removal of the stratum corneum through tape stripping prior to applying PN directly onto the skin for ten consecutive days. Two weeks later, mice are challenged with the intraperitoneal administration of $\mathrm{PN}$. C57BL $/ 6$ and mice deficient in IL-1 $\alpha$, IL-1R1, IgE and mast cells (MC) were used. Clodronate-containing liposomes and anti-CD16/ 32 were used for macrophage $(\mathrm{M} \phi)$ depletion and IgG1-signalling blockade respectively. Peritoneal $\mathrm{M} \phi$ were stimulated in vitro with either calcium ionophore (positive control) or immune complexes (ICs) and cell lysates were analyzed for cytoplasmic phospholipase A2 ( $\left.\mathrm{CPLA}_{2}\right)$ and phosphorylated $\mathrm{cPLA}_{2}$.

Results: PN-specific $\mathrm{IgG}_{1}$ levels were elevated in skin-sensitised mice whereas $\mathrm{PN}$ specific IgE levels were almost undetectable. Wild type (WT), IgE-deficient and MC KO mice underwent severe anaphylaxis. In stark contrast, blockade of $\mathrm{IgG}_{1}$ signalling or $\mathrm{M} \phi$ depletion in WT mice dramatically reduced the anaphylactic reaction. While IL- $1 \alpha \mathrm{KO}$ mice could be sensitised, they did not exhibit anaphylaxis. IL1R1 KO mice and WT mice treated with anti-IL- $1 \alpha$ underwent full anaphylaxis suggesting an intracellular role for IL-1 $\alpha$. Lastly, cPLA2 phosphorylation was observed in peritoneal $\mathrm{M} \phi$ from WT mice exposed to either calcium ionophore or ICs but not in $\mathrm{M} \phi$ from IL-1 $\alpha \mathrm{KO}$ mice.

Conclusion: In this model of epicutaneous sensitisation, anaphylaxis is mainly driven by an $\mathrm{IgG}_{1}-\mathrm{M} \phi$ pathway. Our data show that IL- $1 \alpha$ plays a critical role in the anaphylactic reaction, and that the effect of IL- $1 \alpha$ is intracellular likely through regulating $\mathrm{CPLA}_{2}$ phosphorylation, and potentially PAF secretion, in $\mathrm{M} \phi$.

\section{1}

Epicutaneous immunotherapy protects mice from food-induced anaphylaxis independent of sensitisation route

Tordesillas, $\mathrm{L}^{1}$; Mondoulet, $\mathrm{L}^{2}$; Benhamou, $\mathrm{PH}^{2}$; Sampson, $\mathrm{HA}^{1}$; Berin, $\mathrm{MC}^{1}$

${ }^{1}$ Icahn School of Medicine at Mount Sinai, Jaffe Food Allergy Institute, New York, NY, United States; ${ }^{2}$ DBV

Technologies, Bagneux, France

Background: Epicutaneous and oral immunotherapy (EPIT and OIT) are being investigated for treatment of food allergy. Outcome of EPIT or OIT may depend on the initial route of sensitisation. We compared the efficacy of EPIT and OIT in prevention of food-induced anaphylaxis in orally or epicutaneously sensitised mice.

Method: $\mathrm{C} 3 \mathrm{H} / \mathrm{HeJ}$ mice $(7-10$ mice per group) were sensitised by feeding ovalbumin (OVA) plus cholera toxin, or by epicutaneous OVA without adjuvant. EPIT was performed using OVA - Viaskin ${ }^{\circledR}$ for 8 weeks, and OIT was administered in drinking water for 8 weeks. Mice were orally challenged with OVA to induce anaphylaxis, measured by change in body temperature. OVA-specific immunoglobulins and basophil activation were measured in blood. Treg induction was assessed in the mesenteric lymph node.

Results: Mice sensitised to OVA by either oral or epicutaneous route responded to oral OVA challenge with anaphylaxis measured by significant drop in body temperature $\left[32.4^{\circ} \mathrm{C}\right.$ (oral), 34.2 (skin) vs $38.0^{\circ} \mathrm{C}$ (naive)]. Mice sensitised by either route were resistant to oral challenge after treatment with EPIT. $0 / 7$ orally sensitised EPIT-treated mice developed anaphylaxis (mean temperature $37.6^{\circ} \mathrm{C}$ ). $0 / 9$ skin sensitised EPIT-treated mice developed anaphylaxis $\left(37.9^{\circ} \mathrm{C}\right)$. In contrast, response to OIT was influenced by sensitisation route. Five of nine orally sensitised OIT-treated mice had anaphylaxis $\left(34.8^{\circ} \mathrm{C}\right)$, compared to $0 / 9$ skin sensitised OIT-treated mice $\left(37.7^{\circ} \mathrm{C}\right)$. Unexpectedly, OVA-IgE, OVA-IgG1, OVA-IgG2a, and basophil activation tests did not correlate with clinical reactivity. Epicutaneous antigen exposure induced a population of LAP-positive Foxp3-negative CD4 + T cells similar to Th3 cells in the mesenteric lymph nodes of orally and skinsensitised mice.

Conclusion: EPIT is as effective or more effective than OIT in protection from food-induced anaphylaxis in mice, depending on initial site of allergic priming. The contribution of gastrointestinal Treg populations to clinical protection is under investigation.

\section{2 Allergy to lipid transfer protein: genetic basis-preliminary results \\ Mezzacappa, $\mathrm{S}^{1}$; Nucera, E1; Ria, $\mathrm{F}^{1}$; Pecora, $\mathrm{V}^{2}$; Rizzi, $A^{1}$; Aruanno, $A^{1}$; Pascolini, $L^{1}$; Colagiovanni, $A^{1}$; Ricci, $\mathrm{AG}^{1}$; Di Rienzo, $\mathrm{A}^{1}$; Centrone, $\mathrm{M}^{1}$; Sikora, $\mathrm{A}^{1}$; Ferraironi, $M^{1}$; Valentini, $M^{1}$; Di Sante, $G^{1}$; Buonomo, $A^{1}$; Schiavi- no, $D^{1}$ \\ ${ }^{1}$ Università Cattolica del Sacro Cuore, Rome, Italy; ${ }^{2}$ Università Cattolica del Sacro Cuore, Allergy, Rome, Italy}

Background: Lipid transfer proteins (LTP), profilins and PR-10 are the most important panallergens in South-Centre Italy.LTP are stable molecules, predominantly present in the fruit peel, which can induce systemic symptoms after ingestion of plant-derived foods. The aim of our study was to assess the genetic basis related to panallergens sensitisation. We wanted to evaluate the possible correlation between HLA-DRB1 haplotypes and food allergy.

Method: We enrolled 47 subjects with the history of adverse reactions after the ingestion of plant-derived foods and allergological evaluation (skin prick tests and quantification of specific $\operatorname{IgE}$ ) positive for some food and at least one panallergen.On the basis of the sensitisation to LTP the patients were divided into two groups: $\mathrm{LTP}+=36 ; \mathrm{LTP}_{-}=11$. We extracted the genomic DNA from each patient and detected the HLA-DRB1 haplotypes using the INNO-LIPA HLA-DRB1 kit.

Results: We compared our results with the literature data about the frequency of HLA-DRB1 haplotypes in South-Centre Italy.We observed a marked prevalence of HLA-DRB1 homozygosity in all examined patients, both positive and negative for LTP $(23 \%)$, compared to general population $(3 \%)$.In addition, the HLA-DRB1 13 was twice as frequent in the LTP + group as it was in the general population whereas it was completely absent in the LTPgroup. On the other hand, alleles 4, 7 and 
15 occurred to be twice more often in LTP-group than in the general population and the LTP + group.

Conclusion: This study shows a high rate of sensitisation to LTP in the South-Centre
Italian population according to the Literature about food allergy in this geographical area, therefore panallergens should be considered a clinically relevant food allergens. Altough further investigations are neces- sary we hypothesize that the alleles $4,7,15$ have a protective role against the allergy to LTP. On the other hand the presence of the haplotype HLA-DR1 13 could be a risk factor for LTP allergy. 


\section{Oral Abstract Session}

\section{OAS 8 - Insights into allergic diseases in primary care}

\begin{abstract}
43
Intranasal corticosteroid treatment failure in allergic rhinitis: assessment of unmet need by measuring shift to multiple therapies

Price, $D^{1,2}$; Scadding, $\mathrm{G}^{3}$; Bachert, $\mathrm{C}^{4}$; Saleh, $\mathrm{H}^{5}$; Nasser, $\mathrm{S}^{6}$; Carter, $\mathrm{V}^{7}$; Von Ziegenweidt, $\mathrm{J}^{2}$; Ryan, $\mathrm{D}^{8}$

${ }^{1}$ University of Aberdeen, Aberdeen, United Kingdom;

${ }^{2}$ Research in Real Life (RiRL) Ltd., Cambridge, United Kingdom; ${ }^{3}$ Royal National Throat Nose and Ear Hospital, London, United Kingdom; ${ }^{4}$ University of Ghent, Ghent, Belgium; ${ }^{5}$ Charing Cross \& Royal Brompton Hospitals, London, United Kingdom; ${ }^{6}$ Addenbrooke's Hospital, Cambridge, United Kingdom; ${ }^{7}$ RiRL, Cambridge, United Kingdom; ${ }^{8}$ University of Edinburgh, Edinburgh, United Kingdom
\end{abstract}

Background: Intranasal corticosteroids (INS) are the most effective allergic rhinitis (AR) treatment. ${ }^{1}$ However, they provide insufficient symptom relief for some patients. An INS efficacy threshold of $60 \%$ reduction in overall nasal symptom score has been identified, above which INS fail to differentiate from placebo. ${ }^{2}$ The burden of this pharmacologic insufficiency has not been fully elucidated. In this study, we numerically describe INS treatment failure in AR patients in real life, and show those patients with insufficient symptom control are currently managed.

Methods: This was a retrospective database study using the Optimum Patient Care Research Database which holds patient data from 354 practices across the United Kingdom. Patients included in the analysis had a recorded AR diagnosis and $\geq 1$ AR therapy prescription during 1st March 2010 to 31st August 2010. Here we focus on those AR patients for whom an INS was the first prescription.

Results: In all, 2197 AR patients were included. INS proved insufficient for $36.1 \%$ of these patients; $32.4 \% \quad(n=712)$ required an add-on therapy and $3.7 \%$ $(n=82)$ changed INS AND received additional therapy. Of those 712 patients who received an add-on to their INS, 75.7\% received one, $22.2 \%$ received two and $2.1 \%$ received 3 add-ons. The most common single medications added on to existing INS therapy were antihistamines $(\mathrm{AH}$; $77.6 \%$ of patients), eye drops $(9.5 \%)$, systemic corticosteroids $(9.3 \%)$ and leukotriene receptor antagonists $(2.8 \%)$.

Conclusion: INS provide insufficient symptom control in about one-third of AR patients who visit their doctor. Combined use of INS and oral $\mathrm{AH}$, the most common multiple therapy regimen prescribed by doctors, is not recommended by ARIA due to lack of clinical data or proven nonsuperiority over INS monotherapy. ${ }^{1}$ There is a need for a faster, more effective AR therapy with proven superiority over current gold standard therapy in direct comparison head to head studies.

References: 1. Bousquet J, et al. Allergy 2008;63(suppl 86):8-160.

2. Meltzer $\mathrm{E}$ et al. Int Arch Allergy Immunol 2013;161:369-77.

\section{4 \\ The factors for persistent atopic dermatitis for the first 3 years of age and after in children with food allergy}

Cavkaytar, O; Arik Yilmaz, E; Buyuktiryaki, B; Uysal Soyer, O; Sackesen, C

Department of Pediatric Allergy, Hacettepe University Medical Faculty, Ankara, Turkey

Background: Atopic dermatitis (AD) usually occurs in the first year of life and resolves in a short span of time but it may take years to resolve in some of the children.

The aim of this study is to find out the influence of food allergy (FA) phenotypes on prognosis of $\mathrm{AD}$ during the first 3 years of life.

Method: Children with FA followed-up at least three years were divided into three groups according to existence and progression of $\mathrm{AD}$; (i) Never $A D$, (ii) transient $A D$ (AD exists before 3 years but doesn't continue after $3 \mathrm{yrs}$ ) and (iii) persistent $A D$ (AD exists before 3 years and continues after 3 years).

Groups were compared in relation to phenotypes of FA, multiple FA, sensitisation to aeroallergens, levels of eosinophil percentage and total IgE.

Results: Egg allergy was more frequent in transient $(81 \%)$ and persistant AD (89\%) groups compared to never AD group (38\%), $P<0.0001$. Isolated cows' milk allergy (CMA) was less frequent in persistent AD group $(7.1 \%)$ compared to transient and $(10.9 \%)$ and never $\mathrm{AD}$ groups (\%25), $P=0.004$. The highest eosinophil percentage belonged to persistent $\mathrm{AD}$ group [median (interquartile range); 6\%
(4-8.2)], however it was 5.8\% (3.1-10) and $4.1 \%(2.3-6.3)$ in transient AD and never AD groups respectively $(P=0.001)$. The sensitisation rate to D.farinae or D.pteronyssinus was higher in persistent $\mathrm{AD}$ group $(18 \%)$ compared to transient $\mathrm{AD}$ group $(3 \%)$. The multivariate logistic regression analysis denoted that IgE-mediated egg allergy was a significant risk factor for persistent $\mathrm{AD}$ (OR:4.9, 95\% CI:1.5-16.9, $P=0.01)$.

Conclusion: Egg allergy is more frequent in food allergic children with $\mathrm{AD}$ and moreover it enhances the risk for the persistence of AD. However this finding is not observed in children with allergies to other foods especially with milk. Egg allergic children show a different pattern of prognosis in relation to AD.

\section{5}

Evaluation of allergic rhinitis and asthma control in a Portuguese Community Pharmacy setting

Lourenco, $\mathrm{O}^{1}$; Calado, $\mathrm{S}^{1}$; Sá-Sousa, $\mathrm{A}^{2}$; Fonseca, JA $\mathrm{A}^{2,3,4}$ CICS - UBI Health Sciences Research Centre, Covilha, Portugal; ${ }^{2}$ Biostatistics and Medical Informatics Department, University of Porto, Porto, Portugal; ${ }^{3}$ Allergy Division, Hospital S. Joao EPE, Porto, Portugal; ${ }^{4}$ CINTESIS - Center for Research in Health

Technologies and Information Systems, Porto, Portugal

Background: Allergic Rhinitis and Asthma (ARA) are frequent respiratory diseases that often coexist, causing a high social and economic impact. It is important to maintain ARA disease control to reduce the disease burden. The purpose of this study was to assess control in patients with one or both pathologies through the application of validated questionnaires at community pharmacies.

Method: A cross-sectional study was conducted in the pharmacies of the Portuguese county of Covilhã (located in the central region of Portugal with about 53000 inhabitants). Subjects between 18 and 70 years old, who presented a prescription for an asthma and/or allergic rhinitis medication, were invited to participate in the study by responding to a questionnaire that included the Control of Allergic Rhinitis and Asthma Test (CARAT). The CARAT is a validated tool to simultaneously assess the control of allergic 
rhinitis and asthma with scores that range from 0 to 30 points, 0 meaning the worst and 30 the best possible control of disease. Results: Of the 224 participants, $58 \%$ were female and the median age was 48.5 years. The median CARAT score was 19 $(\mu=17.8 \pm 6.4)$ and $87 \%$ of participants had a score $<25$, indicating non-controlled disease. Female participants, the elderly and those with less school education, responded with scores that demonstrated significantly less disease control.

Conclusion: Using a simple self-assessment questionnaire, such as the CARAT, pharmacists can help identify patients with uncontrolled ARA disease, which is an important first step to change patients' knowledge about their disease with an ultimate goal of improving ARA outcomes.

46

Pharmacists can help to detect undiagnosed moderate-severe respiratory allergy. An EFA pilot study in Vienna, Austria

Liebl, $\mathrm{M}^{1}$; Hafner, $\mathrm{V}^{2}$; Spranger, $\mathrm{O}^{3}$; Fink-Wagner, $\mathrm{AH}^{4}$; Valovirta, $\mathrm{E}^{4}$

${ }^{1}$ Pharmaceutical Group of the European Union, Brussels, Belgium; ${ }^{2}$ Landesgeschäftsstelle Wien, Österreichische Apothekerkammer, Wien, Austria; ${ }^{3}$ Österreichische Lungenunion, Wien, Austria;

${ }^{4}$ European Federation of Allergy and Airways Diseases Patients? Associations (EFA), Brussels, Belgium

Background: Many patients with respiratory allergy do not recognise their condition and fail to consult a physician. Others tend to use proven or unproven means to relieve their symptoms (ARIA Guidelines for Pharmacists). As trusted health care professionals in the community, pharmacists can play a major role in early identification of the condition and guide patients to a correct diagnosis.

Aims: The EFA Pilot Project with Austrian Pharmacists, in collaboration with the Pharmaceutical Group of the European Union and the Austrian Lungenunion, aimed at evaluating the role of pharmacists in the early identification of customers with respiratory allergy by using the validated Allergy Screening Test ASF Questionnaire (Fischer, Allergologie, JG 29, 2006).

Methods: Between April and June 2013 (high allergy season in Austria), 315 pharmacies in Vienna were invited to propose the questionnaire to customers with symptoms of respiratory allergy or asking for OTC treatment for allergic symptoms. Each pharmacy received an 'Allergy Risk Check' box containing: allergy screening questionnaires; instructions how to use it and information on the campaign; the ARIA Guidelines for Pharmacists in German; The EFA Book on Respiratory
Allergies in German; pollen calendars; addresses of allergy centres and doctors providing allergy tests in the Vienna region.

Results: 2297 questionnaires were collected. $76 \%$ of participants were at a moderate-severe risk of allergy. But only $35 \%$ of the total had been tested for allergy; $57 \%$ of the never tested participants 'clearly felt unwell'. Pharmacists advised 1130 participants $(49.2 \%$ of the total) to consult a physician.

Conclusions: Pharmacists are often the first health care professionals seeing a person at risk of respiratory allergy. They can motivate at-risk subjects to consult a physician to receive an early and correct diagnosis. Although larger surveys are needed to verify these results, the Austrian 'Allergy Risk Check' programme can serve as a best practice model for other European countries.

\section{7 \\ Familiarity with the clinical picture and treatment methods of hymenoptera venom allergy (HVA) by Polish general practitioners (GPs)}

Hoscilo, $\mathrm{P}^{1}$; Staron, $\mathrm{G}^{1}$; Hans-Wytrychowska, $\mathrm{A}^{2}$; Barg, $\mathrm{W}^{3}$; Nittner-Marszalska, $\mathrm{M}^{4}$

${ }^{1}$ Department and Clinic of Internal Diseases, Geriatry and Allergology, Wroclaw Medical University, Students' Scientific Association, Wroclaw, Poland; ${ }^{2}$ Department of Family Medicine, Wroclaw Medical University, Wroclaw, Poland; ${ }^{3}$ Department of Physiology, Wroclaw Medical University, Wroclaw, Poland; ${ }^{4}$ Department and Clinic of Internal Diseases, Geriatry and Allergology, Wroclaw Medical University, Wroclaw, Poland

Background: The role of GPs in Europe, including Poland, has significantly increased in the past few decades. A welltrained GP is able to diagnose and treat the majority of typical allergic conditions. However, in more severe and problematic cases such as HVA, it is GPs who refer patients to specialists and therefore have to be familiar with the clinical picture and treatment methods of HVA. The study is aimed to estimate the knowledge of HVA among GPs in Poland.

Method: GPs attending Top Medical Trends 2013 in Poznan (Poland) were requested to fill in a questionnaire composed of five items assessing doctors' knowledge of the clinical aspects of HVA, as well as some selected aspects of HVA treatment. The questionnaire was constructed according to the guidelines and recommendations of the European Academy of Allergy and Clinical Immunology (EAACI).

Results: 308 questionnaires were distributed, 275 were returned and approved for further analysis (the audit response rate was $89.29 \%$ ). All respondents were GPs specialising in family medicine (43.64\%) and internal medicine (42.18\%). $99.6 \%$ of the respondents declared sufficient knowledge of HVA symptoms. However, only in $2.91 \%$ of the returned questionnaires the answers concerning the issue were all correct. The most common mistakes were: to assume erythema and pain at the site of insect stinging as characteristic symptoms for $\mathrm{LL}$ reaction $(91.64 \%)$ and to assume that increase of blood pressure $(63.64 \%)$ and lymphadenopathy $(58.91 \%)$ are symptoms of insect allergy. Most of the respondents knew that venom immunotherapy (VIT) is the method of treatment for patients with HVA. Unfortunately, only $7.27 \%$ answered correctly to the question about indications for VIT and only $46.97 \%$ knew that VIT is very effective.

Conclusion: The questionnaire revealed two major 'sins' of Polish GPs with regard to their familiarity with HVA problematics: likely over-diagnosis of HVA on the one hand and underestimation of the effectiveness of VIT on the other. Both can have negative effects for HVA patients.

\section{8 \\ Determination of the minimal clinically important difference in symptom score in seasonal allergic rhinitis: a prospective study in primary care}

Devillier, $\mathrm{P}^{1}$; Le Neuder, $\mathrm{G}^{2}$; Dreyfus, J- $\mathrm{F}^{3}$; Bousquet,

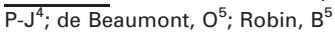

${ }^{1}$ Foch Hospital, UPRES EA 220 \& Clinical Research Department, University of Versailles Saint-Quentin, Suresnes, France; ${ }^{2}$ Universal Medica, Saint-Cloud, France; ${ }^{3}$ Biostatistic Unit, Clincal Research

Deapartment, Foch Hospital, Suresnes, France; ${ }^{4}$ Hopital Arnaud de Villeneuve, University Hospital of Montpellier, Montpellier, France; ${ }^{5}$ Stallergenes S.A., Antony, France

Background: The minimal important difference (MID) represents the smallest improvement considered meaningful by a patient. In allergic rhinitis (AR), the MID has been established for the Rhinoconjunctivitis Quality-of-Life Questionnaire (RQLQ) whereas the main criterion of treatment efficacy is the rhinoconjunctivitis total (nasal +ocular) symptom score (RTSS). The main objective of this study was to determine the MID of the RTSS for individuals over time.

Methods: This multicenter prospective study was conducted in grass pollen allergic patients consulting an allergy specialist. RTSS was assessed at the consultation day and then daily and weekly for a 15-point global rating of change scale (GRCS) and the RQLQ during four weeks. MID of the RTSS was determined by the anchor-based (using +2 as the minimal improvement in the GRCS and 0.5 point as the minimal improvement in the RQLQ) and the 
distribution-based (using the standard deviation (0.33 SD) of the RTSS) methods. Estimates of the MID from these two methods have been used to define the MID.

Results: The primary analysis was performed on the data obtained in the first fortnight of the study for 806 patients (253 children, 250 adolescents, and 303 adults) with persistent moderate/severe rhinitis $(84 \%)$ according to ARIA classification.
During the 1st week of the study, the mean \pm SD average RTSSs for children, adolescents and adults were $6.5 \pm 3.3,6.8 \pm 3.4$, and $7.0 \pm 3.4$, respectively. The MID in the RTSS based on the RQLQ was determined by regression analysis to be $1.12 \pm 0.14, \quad 1.20 \pm 0.13$ and $0.89 \pm 0.12$ in children, adolescents and adults, respectively. Taking +2 as the minimal improvement in the GRCS score, the MID in the RTSS (mean $\pm \mathrm{SD}$ ) was $1.24 \pm 0.17$,
$1.33 \pm 0.10$ and $1.13 \pm 0.14$ in children, adolescents and adults, respectively. Using the distribution-based method (0.33 SD), the MID of the RTSS was between 1.09 and 1.13 .

Conclusion: For patients with grass polleninduced AR being treated in primary care, the MID in the RTSS is approximately 1.2 in patients with moderate-to-severe grass pollen AR. 


\section{Oral Abstract Session}

\section{OAS 9 - Innovations in hymenoptera venom allergy}

49

Analysis of $\lg E$ and $\lg G 4$ profiles to a panel of CCD free bee venom allergens in bee venom allergic patients treated with venom immunotherapy

Frick, $\mathrm{M}^{1}$; Bantleon, $\mathrm{F}^{2}$; Rafei-Shamsabadi, $\mathrm{D}^{1}$; Müller, $\overline{\mathrm{S}^{1} \text {; Blank, }} \mathrm{S}^{3}$; Huss-Marp, $\mathrm{J}^{1,4}$; Rueff, $\mathrm{F}^{5}$; Helbling, $\mathrm{A}^{6}$; Spillner, $E^{2}$; Jakob, $T^{1}$

${ }^{1}$ Department of Dermatology, University Medical Center Freiburg, Allergy Research Group, Freiburg, Germany; ${ }^{2}$ Department of Engineering, Aarhus University, Aarhus, Denmark; ${ }^{3} \mathrm{TU}$ and Helmholtz Center Munich, Center of Allergy and Environment (ZAUM), Munich, Germany; ${ }^{4}$ Thermo Fisher Scientific, Freiburg,

Germany; ${ }^{5}$ Department of Dermatology and Allergology, Ludwig-Maximilians-University Munich, Munich, Germany; ${ }^{6}$ Division of Allergy, SpitalNetz Bern Ziegler, Bern, Switzerland

Background: Detection of IgE to recombinant (r) Hymenoptera venom allergens has been suggested to improve the diagnostic precision in Hymenoptera venom allergy. Using a panel of CCD free bee venom allergens (rApi m 1, rApi m 2, rApi m 3, nApi $\mathrm{m} \mathrm{4,} \mathrm{rApi} \mathrm{m} 5$ and rApi $\mathrm{m} \mathrm{10)}$ we recently demonstrated improved diagnostic sensitivity as compared to use of rApi $\mathrm{m} 1$ alone, identified Api m 3, Api m 5 and Api $\mathrm{m} 10$ as additional major allergens and revealed distinct sensitisation profiles some of which are dominated by $\operatorname{IgE}$ to allergens that have been reported to be absent or underrepresented in therapeutic HBV preparations.Here we now report the IgE and IgG4 profiles of bee venom allergic patients before and under treatment with venom immunotherapy (VIT).

Methods: Diagnosis of bee venom allergy $(n=56)$ was based on history, skin test and $\operatorname{sIgE}$ to bee venom extract (i1). $\operatorname{IgE}$ reactivity was analysed to the entire panel of $\mathrm{HBV}$ allergens, IgG4 reactivity was analysed to rApi m 1 (i208), rApi m 3, nApi $\mathrm{m} \mathrm{4}$, and rApi $\mathrm{m} 10$ using ImmunoCAP research prototypes (Thermo Fisher Scientific, Uppsala, Sweden).

Results: The IgE sensitisation frequency to rApi m 1,rApi m 2, rApi m 3, nApi m 4, rApi $\mathrm{m} 5$ and rApi m 10 was $80.3 \%$, $64.3 \%, 46.4 \%, 32.1 \%, 67.9 \%$ and $77.4 \%$ respectively and confirmed previously reported frequencies. The analysis before and after at least 6 months of VIT demonstrated a more than 2 fold increase in $\operatorname{IgE}$ reactivity to rApi $\mathrm{m} \mathrm{1,} \mathrm{rApi} \mathrm{m} 2$, rApi $\mathrm{m}$ 3, nApi $\mathrm{m} 4$ and rApi $\mathrm{m} 10$ in $17.9 \%$, $21.4 \%, 7.1 \%, 14.3 \%$, and $8.9 \%$ respec- tively. VIT induced IgE levels above the cutoff $(>0.35 \mathrm{kU} / \mathrm{l})$ in previously negative patients in 4/11 for Api m 1, 5/20 for Api m 2, 2/30 for Api m 3, 5/38 for Api m 4 and $1 / 16$ for Api m 10. Analysis of sIgG4 demonstrated that VIT induced a pronounced IgG4 response to HBV $(9.4 \pm 15.2$ fold $)$, Api $m 1(22.8 \pm 59.2$ fold) and Api m 4 (11.9 \pm 26.7 fold), while only moderate induction of $\mathrm{IgG} 4$ to Api m 3 (1.6 \pm 1.4 fold $)$ and Api $m 10(2.6 \pm 3.6$ fold) was observed.

Conclusion: Our data confirms the presence of a broad spectrum of different sensitisation profiles. It demonstrates a certain degree of new sensitisations to single allergens during VIT, a robust induction of allergen specific IgG4 responses to high abundance allergens and a relative lack to allergens that have been reported to be absent or underrepresented in therapeutic HBV preparations. Future analysis will have to address the relevance of these parameters for the outcome of VIT.

\section{0}

Recombinant Polistes dominula protease allergen Pol d 4 as candidate for dissection of sensitisation to vespid venoms

Wolf, $\mathrm{S}^{1}$; Blank, $\mathrm{S}^{2}$; Bantleon, $\mathrm{F}^{1}$; Jakob, $\mathrm{T}^{3}$; Spillner, $\mathrm{E}^{1}$ Department of Engineering, Aarhus University, Aarhus, Denmark; ${ }^{2}$ Technical University and Helmholtz Center Munich, Center of Allergy and Environment (ZAUM), Munich, Germany; ${ }^{3}$ Department of Dermatology, Medical Center - University of Freiburg, Allergy Reserch Group, Freiburg, Germany

Background: Sensitisation to cross-reactive allergens poses significant problems in Hymenoptera venom allergy. Molecular and structural similarity of vespid venom allergens from Vespula and the increasingly spreading Polistes species makes reliable identification of culprit venom and therapeutic decision highly difficult. Species-specific and CCD-free allergens could provide opportunities to dissect sensitisation of venom allergic patients.

Objective: Recombinant production of the heavily glycosylated putative protease allergen Pol d 4, analysis of cross-reactivity with other venom proteases and analysis of sensitisation of venom allergic patients.

Method: Recombinant Pol d 4 was cloned and expressed via baculovirus-mediated infection of insect cells. The purified protease was assessed for the presence of CCDs and specific IgE reactivity of venom-sensitised patients as compared to Pol d 5, Ves $\mathrm{v} 1$ and Ves v 5. Diagnosis of venom allergy was based on history, skin test and sIgE to venom. Moreover, we built homology models of Pol d 4 and other putative venom proteases.

Results: Recombinant Pol d 4 could be produced in high yields as secreted protein in Sf9 insect cells. The purified, highly glycosylated protein exhibited no detectable CCD reactivity. Molecular modeling of Pol d 4 revealed a typical fold of serine proteases and emphasized the reduced molecular similarity with other putative venom proteases. Using the recombinant Pol d 4 sIgE reactivities of venom allergic patients were assessed.

Conclusion: Recombinant and CCD-free Pol d 4 might provide a valuable tool for differentiating Polistes and Vespula venom sensitisation.

51

Important role of hyaluronidase in case of protein based cross-reactivity in Hymenoptera venom allergy

Celesnik Smodis, $\mathrm{N}^{1}$; Mittermann, $\mathrm{I}^{2}$; Silar, $\mathrm{M}^{1}$; Kopac, $\mathrm{P}^{1} ;$ Kosnik, $\mathrm{M}^{1}$; Valenta, $\mathrm{R}^{2}$; Korosec, $\mathrm{P}^{1}$ ${ }^{1}$ University Clinic of Respiratory and Allergic Diseases Golnik, Golnik, Slovenia; ${ }^{2}$ Department of Pathophysiology/Division of Immunopathology, Christian Doppler Laboratory for Allergy Research, Center for Physiology and Pathophysiology, Medical University of Vienna, Vienna, Austria

Background: The role of hyaluronidase in Vespula allergy has been recently reassessed and recognised as being modest. Moreover the majority of its reactivity has been assigned to CCDs and not to the protein itself. On the other hand evaluation of differently fucosylated honeybee hyaluronidases revealed allergenic relevance beyond its carbohydrate epitopes.

Method: Fourteen Hymenoptera venom allergic patients with an unknown culprit insect and double-positive sIgE and BAT to Vespula and honeybee whole venom extracts were included in the study. We evaluated IgE reactivity to honeybee hyaluronidases - rApi m 2, expressed in E. coli, Pichia and insect cells (HighFive - glycosy- 
lated and Sf3 - without alpha-1,3-core fucosylation) and to Sf3-expressed Vespula hyaluronidase $-\mathrm{rVes} v 2$. Furthermore in all patients Sf3-expressed rApi m 2 was evaluated with CD63 basophil response for allergenic activity. Protein-based reactivity was also tested with $\operatorname{IgE}$ inhibition.

Results: IgE reactivity to rApi $m 2$ expressed in Sf3, Pichia, E. coli and HighFive was demonstrated in $10,9,8$ and 12 patients, respectively and to $\mathrm{Sf} 3$-expressed rVes $v 2$ in five patients. IgE reactivities of Sf3 and Pichia-expressed rApi m 2 showed an excellent agreement with basophil response ( $\kappa .84$ and .82 , respectively) and E. coli-expressed a moderate agreement $(\kappa$ .57), whereas highly glycosylated HighFive showed only a slight agreement ( $\kappa$.10). Basophil response with Sf3-expressed rApi m 2 showed a very high allergenic activity in all nine positive patients, with median $\mathrm{EC} 15$ of $0.2 \mathrm{ng} / \mathrm{ml}$. Protein-based reactivity to rApi $\mathrm{m} 2$ or rVes $\mathrm{v} 2$ was furthermore confirmed in five out of seven patients with inhibition tests.

Conclusion: Protein-based sensitisation to hyaluronidase seems to be a frequent cause for double positivity to honeybee and Vespula venom obtained with whole venom extracts. Furthermore we observed high allergenic activity of rApi $\mathrm{m} 2$, which indicates clinical importance of this sensitisation.

52

Oxidative stress markers in patients with hymenoptera venom allergy

Patella, $\mathrm{V}^{1}$; Incorvaia, $\mathrm{C}^{2}$; Minciullo, $\mathrm{PL}^{3}$; Oricchio, $\mathrm{C}^{4}$; Saitta, $\mathrm{S}^{3}$; Florio, $\mathrm{G}^{1}$; Saija, $\mathrm{A}^{5}$; Gangemi, $\mathrm{S}^{3,6}$ ${ }^{1}$ Division of Allergy and Clinical Immunology, Internal Medicine, ASL SALERNO, Battipaglia, Salerno, Italy; ${ }^{2}$ Allergy/Pulmonary Rehabilitation, ICP Hospital, Milan, Italy; ${ }^{3}$ School and Division of Allergy and Clinical Immunology, Department of Clinical and Experimental Medicine, University of Messina, Messina, Italy; ${ }^{4}$ Unit of Transfusion Medicine and Immunohematology, ASL SALERNO, Hospital of Polla, Salerno, Italy; ${ }^{5}$ FarmacoBiologico Department, School of Pharmacy, University of Messina, Messina, Italy; ${ }^{6}$ IBIM (Istituto di

Biomedicina e Immunologia Molecolare), CNR (Consiglio Nazionale delle Ricerche), Palermo, Italy

Background: Oxidative stress occurs in many allergic and immunologic disorders as a result of the imbalance between the endogenous production of free reactive oxygen species (ROS) and/or the reduction of antioxidant defence mechanisms. Advanced glycation end products (AGEs) and advanced oxidation protein products (AOPPs) are compounds formed by transformation of macromolecules, including proteins, which can be used as densitometric markers of oxidative stress and inflammation.

Objective: To examine the serum levels of AGE, AOPP and nitrosylated proteins
(NP) in patients with hymenoptera venom allergy before and after ultrarush venom immunotherapy (VIT).

Methods: The study included 30 patients undergoing VIT by aqueous preparation of Vespula Species (26 pts) or Apis Mellifera (4 pts) from Stallergenes (Antony, France). Blood samples were collected to measure at baseline (T1), at the end of the incremental phase of the VIT protocol (T2) and after 15 days (T3). A group of healthy subjects served as control.

Results: AOPP levels at T1 were significantly higher compared with controls $(P=0.001)$, while levels of NP at T1 were significantly lower than in controls $(P<0.0001)$. No significant differences in levels of AGE were found.

Conclusions: These findings suggest that, despite hymenoptera venom allergy is clinically expressed by isolated episodes of reactions to stings and is not included in chronic inflammatory disorders, an oxidative stress occurs in patients with such hypersensitivity, as indicated by higher levels of serum AOPPs and lower levels of NP compared with controls. No significant changes were detected at short-term interval following venom immunotherapy, but an effect over time cannot be excluded.

\section{3}

Asymptomatically sensitised subjects show stronger slgE/slgG4 responses after sting challenges compared to subjects with clinically relevant sensitisation after field stings

Bokanovic, D; Schwarz, I; Schuster, C; Laipold, K; Aberer, W; Sturm, GJ

Department of Dermatology and Venereology, Medical University of Graz, Graz, Austria

Introduction: To date no serological markers have been found being able to predict the occurrence and severity of future sting reactions. In a previous study we were able to show that only $4.6 \%$ of asymptomatically sensitised subjects developed systemic reactions in sting challenges, which is slightly more than the prevalence of $3.3 \%$ of hymenoptera venom allergy in our area. Our aim was to evaluate potential differences of sIgE and sIgG4 levels after stings in asymptomatically sensitised subjects and hymenoptera venom-allergic patients with and without immunotherapy.

Methods: 186 subjects were included. Of those, 77 were asymptomatically sensitised, 59 were included immediately after a systemic sting reaction, and 50 patients had received venom immunotherapy (VIT). Levels of total IgE, sIgE and sIgG4 to bee and wasp venom were detected before the sting challenge in asymptomatic patients and patients under VIT; in acute patients immediately after the field sting. After 4 weeks, sIgE and sIgG4 levels were reassessed.

Results: Patients under VIT with bee or wasp venom showed no significant increase in $\mathrm{sIgE}$ and sIgG4 after sting challenges with the respective insect. However, in subjects asymptomatically sensitised to bee venom, there was a 4.3 -fold increase in SIgE $(P<0.001)$ and a 2.4 -fold increase in sIgG4 $(P<0.001)$. In asymptomatic subjects sensitised to wasp venom, increases were 2.0 -fold $(P<0.001)$ and 1.8 -fold $(P<0.001)$ respectively.

After systemic sting reactions to wasp stings increases of $\operatorname{sIgE}$ and $\mathrm{sIgG} 4$ to wasp venom were 2.2 -fold in $\operatorname{sigE}(P=0.023)$ and 4.4-fold in sIgG4 $(P<0.001)$. No significant increase of sIgE and sIgG4 could be detected after sting reactions to bee stings.

Interestingly, there was a significant difference between untreated allergic and asymptomatic subjects in the baseline ratio of $\mathrm{sIgE} / \mathrm{sIgG} 4$ to bee venom $(P=0.034)$. The differing baseline ratio of $\mathrm{sIgE} / \mathrm{sIgG} 4$ to wasp venom between those two groups showed a statistical trend $(P=0.066)$.

Conclusion: Patients with ongoing immunotherapy showed no increase in sIgE and sIgG4 after sting challenges. In contrast, asymptomatically sensitised subjects had a considerable increase in sIgE and sIgG4 after the tolerated sting challenge. In venom-allergic patients, sIgE and sIgG4 significantly increased only after systemic reactions to wasp stings but not to bee stings.

\section{4 \\ Factors associated with side effects during the build-up phase of honeybee venom immunotherapy}

Korosec, P; Ziberna, K; Silar, M; Dezman, M; Smodis

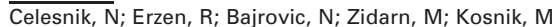
University Clinic of Respiratory and Allergic Diseases Golnik, Golnik, Slovenia

Background: Adverse systemic reactions (SRs) are more common during the honeybee then the wasp venom immunotherapy (VIT). Our aim was to evaluate risk factors for SRs during the build-up phase of honeybee venom immunotherapy.

Method: In this prospective single-center observational study we included 93 patients with established honeybee venom allergy who underwent ultra-rush honeybee VIT. The SRs and their severity were classified according to the WAO grading system for SCIT SRs and then compered to different immunological parameters (sIgE, tIgE, basophil CD63 response, baseline 
tryptase, skin prick tests), patients- specific factors (age, sex, cardiovascular conditions and medications, other allergic diseases) and details of the honeybee field stinginduced anaphylaxis (severity, time interval to onset of symptoms, absence of cutaneous symptoms).

Results: Twenty-three patients $(24.5 \%)$ experienced mild SRs and 13 patients severe SRs. Three significant risk factors for SRs were identified: basophil CD63 response, time interval to onset of symptoms after the sting and sIgE to rApi $\mathrm{m} 1$. The most significant and highly related with the severe grades of SRs was an increased basophil allergen CD63 response evaluated as allergen dose-response metrics of EC15, EC50, CD-sens and area under the curve, or the response to single $0.01 \mu \mathrm{g} / \mathrm{ml}$ of venom concentration. The time interval of less than 5 min from filed sting to onset of symptoms was also related with severe SRs. Specific IgEs to rApi $\mathrm{m} 1$ were lower in patients with SRs. We could not provide the evidence for other study factors.

Conclusion: Increased basophil allergen CD63 response and short latency to filed sting reaction were the indicators of severe adverse SRs during the build-up phase of honeybee VIT. Possibly role was also showed for low sIgE to rApi m1. 


\section{Oral Abstract Session}

\section{OAS 10 - Etiopathogenic mechanisms in chronic rhinosinusitis}

55

TMEM16A-mediated mucin production in IL-13-induced nasal epithelial cells from chronic rhinosinusitis patients

Zhang, $Y^{1}$; Wang, $\mathrm{X}^{1,2}$; Wang, $\mathrm{H}^{2}$; Jiao, J' $\mathrm{J}^{2}$ Li, $\mathrm{Y}^{2} ;$ Fan, $\bar{E}^{2} ;$ Zhang, $\mathrm{L}^{1,2} ;$ Bachert, $\mathrm{C}^{3}$

'Beijing Tongren Hospital, Capital Medical University, Beijing, China; ${ }^{2}$ Key Laboratory of Otolaryngology, Head and Neck Surgery, Ministry of Education, Beijing Institute of Otolaryngology, Beijing, China; ${ }^{3}$ Department of Oto-Rhino-Laryngology, Upper Airways Research

Laboratory, Ghent University Hospital, Ghent, Belgium

Background: Chronic rhinosinusitis with nasal polyps (CRSwNP), a Th2 cytokinemediated disease, often involves mucus oversecretion. Recent evidence suggests that TMEM16A, a membrane protein linked with the activity of $\mathrm{Ca}^{2+}$-activated $\mathrm{Cl}^{-}$channels (CaCCs), can regulate mucus secretion from airway epithelium by transepithelial electrolyte transport and hydration. However, the role of TMEM16A in mucin production/secretion in the airway epithelium is not clear.

Method: To determine TMEM16A mediated mucin production in human nasal polyp epithelial cells (HNPECs) induced by IL-13, human sinonasal mucosa tissues and dissociated sinonasal epithelium from control subjects and patients with CRSwNP were assessed for expression of TMEM16A and MUC5AC by immunohistochemistry and western blot analysis. A model of the Th2 inflammatory environment was created by exposure of primary air-liquid interface (ALI) cultured HNPECs to interleukin-13 (IL-13) for 14 days, with subsequent assessment of TMEM16A and MUC5AC expression.

Results: Expression of TMEM16A and MUC5AC was increased in human nasal polyp tissues and dissociated nasal polyp epithelium. TMEM16A was detected specifically in MUC5AC-positive cells but not in ciliated cells in IL-13 treated HNPECs. IL-13 treatment increased the percentage of TMEM16A-positive cells, the percentage of cells co-expressing TMEM16A and MUC5AC, and the expression of TMEM16A and MUC5AC proteins.T16 $\mathrm{A}_{\mathrm{inh}}-01$, a TMEM16A inhibitor, attenuated the IL-13-induced effects.

Conclusion: Expression of TMEM16A and MUC5AC is increased in CRSwNP, which might be a direct effect of Th2 cytokines present in the sinonasal mucosa in
CRSwNP. Down-regulation of TMEM16A and MUC5AC expression of HNPECs by $\mathrm{T}_{16 \mathrm{~A}_{\mathrm{inh}}-01}$ indicates that TMEM16A might be an important role in mucin production.

56

Pattern of expression of epithelial proteins and physiological recording of three dimensional highly morphologic cultured nasal mucosa

Kirsche, H; Fraenzer, J-T; Cramer, J-M; Rudack, C ENT, University Hospital Muenster, Muenster, Germany

Background: In order to characterise immunohistochemically epithelial proteins of structurally intact nasal tissue samples of three different groups of patients as a basis for comparative studies after transfection (e. g. with $S$. aureus) in a three-dimensional cell culture, transepithelial resistance (TEER) was additionally determined.

Method: Investigated were 15 tissue samples from patients with and without nasal polyps (CRSwNP/CRSsNP) and 14 nasal turbinates of patients without signs of inflammation (controls) in tissue microarrays (TMA). Immunohistochemically graduated (no/weak vs medium/strong staining) quantitative analysis of E-cadherin, Claudin 1, 4 and 7, Occludin 1, Zonula occludens 1 (ZO-1), Desmoglein 2, Junctional adhesion molecule A (JAM-A) and Peroxisome proliferator-activated receptor alpha (PPAR-alpha) were performed. From these samples, three-dimensional cell cultures were performed in parallel and analysed via qRT-PCR and determination of TEER.

Results: Weak expression of Occludin 1 and a pronounced expression of $\mathrm{ZO}-1$ in basal cells and Claudin 7 could be shown immunohistochemically across all three groups. No considerably differences were found for E-Cadherin, Claudin 1, 4 and 7, Occludin 1 and ZO-1 between the three groups. Desmoglein 2 showed in CRSsNP a weak expression compared to controls and CRSwNP. First results of cell culture showed differences of TEER between the three groups. Furthermore, data of qRTPCR of differentially regulated genes will be presented.
Conclusion: The results show various occurrence of structural proteins in nasal mucosa, which differ between controls, CRSsNP and CRSwNP immunohistochemically and concerning the TEER. A threedimensional cell culture could be established, which allows dynamic investigations of protein expression.

57

Programmed cell death 1 (PD-1) overexpression in chronic rhinosinusitis with nasal Polyps correlates with radiologic severity of disease

Kortekaas Krohn, $I^{1}$; Bobic, $S^{1}$; Steelant, $B^{1}$; Van Gerven, $\mathrm{L}^{1}$; Liston, $\mathrm{A}^{2}$; Ceuppens, $\mathrm{J}^{1}$; Hellings, $\mathrm{P}^{1,3}$ ${ }^{1}$ Microbiology and Immunology, Lab for Clinical Immunology, KU Leuven, Leuven, Belgium; ${ }^{2}$ Laboratory of Genetics of Autoimmunity, KU Leuven, Leuven, Belgium; ${ }^{3}$ Otorhinolaryngology, UZ Leuven, Leuven, Belgium

Background: The immunologic role of Programmed cell Death 1 (PD-1) expression and the ligands PD-L1 and PD-L2 in chronic rhinosinusitis with nasal polyps (CRSwNP) is currently not well defined. PD-1 is a negative regulator of $\mathrm{T}$ cell responses by inhibiting $\mathrm{T}$ cell proliferation and cytokine production. The PD-1/PD-L1 or L2 interaction may play an important pathophysiologic role in CRSwNP, where low TGF- $\beta$ concentrations have been considered as a sign of defective regulatory $\mathrm{T}$ cell activity.

Method: Expression levels of PD-1, PDL1, PD-L2 and TGF- $\beta$ were measured by real time PCR on homogenates of CRSwNP $(n=21)$ and control inferior turbinate tissue $(n=21)$. Disease severity was scored radiologically by using the LundMackay scores of maxillo-facial CT scans. The expression levels of PD-1 and ligands were compared with the total CT scan scores and to the mRNA expression levels of TGF- $\beta$.

Results: In CRSwNP, increased mRNA expression of PD-1 (38.98 vs 1.32, $P=0.0005)$ and reduced PD-L1 (0.54 vs $1.06, P=0.020)$ and PD-L2 (0.36 vs 0.93 , $P=0.003)$ mRNA levels were found compared to normal nasal tissue. The ratios of PD-1/PD-L1 and PD-1/PD-L2 in CRSwNP were significantly higher compared to controls $(P=0.0007$ and 0.0006 
respectively). In CRSwNP, the relative mRNA expression of TGF- $\beta$ was decreased compared to normal nasal tissue (6.49 vs $8.50, P=0.042$ ).

The relative mRNA expression of PDL1 correlated with the TGF- $\beta$ expression in nasal polyp tissue $(r=0.53, P=0.023)$. The relative mRNA expression of PD-1 correlated with the total CT scan score $(r=0.50, P=0.021)$, and a negative correlation of PD-L1 with the total CT scan score was found ( $r=-0.47, P=0.032$ ).

Conclusion: We here show the involvement of PD-1 and its ligands in CRSwNP. The correlation of PD-1 expression with radiologic assessment of the degree of sinus opacity suggests an important role in the pathophysiology of CRSwNP.

\section{8 \\ Expression profiles of matrix metalloproteinases and their inhibitors in nasal polyps}

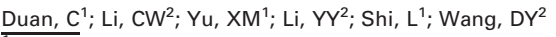
Oilu Hospital, Otolaryngology, Shandong University, Jinan, China; ${ }^{2}$ Otolaryngology, National University of Singapore (NUS), National University Health System (NUHS), Singapore City, Singapore

Background: Increase of inflammatory cell infiltration is significant in nasal polyps (NPs). Matrix metalloproteinases (MMPs) are considered to play an important role in leukocyte migration during the airway inflammation. Our previous microarray study has identified the up-regulation of MMP1, MMP9, MMP10, and MMP12, but down-regulation of the tissue inhibitor of metalloproteinase (TIMP) 1 and 3 in NPs. We sought to further confirm the expression levels of MMPs and TIMPs and study the relationship between the inflammatory cells and MMPs/TIMPs in NPs.

Method: NP tissues from 51 Chinese patients with bilateral polyps were obtained during the surgery. Inferior turbinate (IT) tissues from 19 patients with septal deviation were served as controls. mRNA and protein levels of MMPs/TIMPs were assessed by quantitative PCR and immunohistochemistry.

Results: Eosinophilia ( $\geq 10 \%$ of total leukocytes) (72\%, 23/32 samples), neutrophilia $(41 \%, 13 / 32$ samples), and increase of macrophages $(38 \%, 12 / 32$ samples)were found in NP tissues. mRNA expression of MMP1 (6.7-fold), MMP9 (4.1-fold), MMP10 (10.8-fold) and MMP12 (3.5-fold) was significantly up-regulated while TIMP1 (1.5-fold) and TIMP3 (6.0-fold) was significantly down-regulated in NPs $(n=51)$ as compared to controls $(n=19)$. The immunostaining levels of all four MMPs and two TIMPs were higher in NPs than in controls. The number of MMP12 positive cells was higher in the eosinophilic NPs, MMP9 positive cells were increased in neutrophilic NPs, while MMP10 positive cells were higher in the samples with increase of macrophages. Furthermore, up-regulation of TIMP1 or TIMP3 positive cells was mostly found in eosinophilic NPs.

Conclusion: These findings indicate that the increase of MMP9, MMP10, and MMP12 in NPs may contribute to the infiltration of neutrophils, macrophages, and eosinophils, respectively. Up-regulation of MMP inhibitors in eosinophilic NPs may suggest a regulatory responses occurring at a site of persistent mucosal inflammation.

\section{9}

Microarray gene analysis of nasal epithelial cells in patients with chronic rhinosinusitis with nasal polyposis

Cornet, $\mathrm{M}^{1}$; Kostamo, $\mathrm{K}^{2}$; Rinia, $\mathrm{AB}^{1}$; van Egmond, $\mathrm{D}^{1}$; Zwinderman, $\mathrm{AH}^{1}$; Fokkens, $\mathrm{WJ}^{1}$; van Drunen, $\mathrm{CM}^{1}$ ${ }^{1}$ AMC, Amsterdam, The Netherlands; ${ }^{2}$ University of Helsinki, Helsinki, Finland

Background: Chronic rhinosinusitis with nasal polyps (CRSwNP) is a multifactorial chronic disease of unknown aetiology. Although, the link between nasal polyposis and asthma suggests a systemic aspect of the disease, polyps form only around the osteomeatal complex. Here we investigate whether epithelial cells from polyposis patients differ from healthy controls and the potential impact of co-morbidities and location within the nose.

Method: We obtained nasal epithelial cells from concha media and sphenoid sinus of ten healthy individuals and concha media and polyps of 29 CRSwNP patients) using a magnetic beat assisted isolation procedure. The group of CRSwNP patients had ten patients without comorbidities, four with only allergy, nine with only asthma, and six with both allergic and asthma. A micro-array was used to determine differences in the expression profile as a function of the disease, the location and the comorbidities.

Results: We find 1022 significant differentially regulated genes with 603 genes expressed higher and 419 genes expressed lower in polyps vs healthy concha. The affected genes belong to expected gene ontology groups 'immune responses' and 'negative regulation of apoptosis'. Interestingly, also multiple biological processes involving 'amino acid biosynthesis' seem affected, which could be related to increase observed in genes associated with 'endoplasmatic reticulum stress' and 'response to unfolded proteins'. In addition we detected a deregulation of multiple transcription factors and miRNAs. Moreover, we find a strong impact of comorbidities on the epithelial expression profile, but interestingly mostly for asthma and less for allergic status.

Conclusion: There are major differences between healthy nasal epithelial cells and the epithelial cells from nasal polyps. A detailed analysis of these differences will contribute to a better understanding of pathological process associated with nasal polyposis. 


\section{Oral Abstract Session}

\section{OAS 11 - Immunogenetics and immunogenomics}

\begin{abstract}
60
A genome-wide association study (GWAS) in patients with urticaria/ angioedema induced by hypersensitivity to multiple non-steroidal anti-inflammatory drugs (NIUA)

Cornejo-García, JA ${ }^{1}$; Lee, $\mathrm{MT}^{2}$; Blanca-López, $\mathrm{NA}^{3}$; Doña, I $^{4}$ Martín-Iglesias, MA ${ }^{5}$; Godineau, $V^{1}$; Fernández, $\mathrm{J}^{6}$; Laguna, $\mathrm{JJ}^{7}$; Ayuso, $\mathrm{P}^{1}$; Plaza-Serón, $\mathrm{MC}^{1}$; Mayorga, $\mathrm{C}^{1}$; Canto, $\mathrm{GA}^{3}$; Torres, $\mathrm{MJ}^{4}$; Blanca, $\mathrm{M}^{4}$

${ }^{1}$ Research Laboratory, IBIMA, Regional University Hospital of Malaga, UMA, Málaga, Spain: ${ }^{2}$ Institute of Biomedical Sciences, Academia Sinica, Taipei, Taiwan ${ }^{3}$ Allergy Service, Infanta Leonor Hospital, Madrid, Spain; ${ }^{4}$ Allergy Unit, IBIMA, Regional University Hospital of Malaga, UMA, Málaga, Spain; ${ }^{5}$ Allergy Service, Santa Barbara Hospital, Puerto Llano, Ciudad Real, Spain; ${ }^{6}$ Allergy Service, Alicante Hospital,

Alicante, Spain; ${ }^{7}$ Allergy \& Asthma Center Service, Cruz Roja Hospital, Madrid, Spain
\end{abstract}

Background: Urticaria/angioedema-induced by multiple non-steroidal anti-inflammatory drugs (NSAIDs) (NIUA) is the most frequent clinical entity caused by hypersensitivity reactions to drugs (HRs). Most studies analyzing the genetic basis of NIUA and related phenotypes have followed the biological plausibility criteria, focusing on polymorphisms in genes belonging to the arachidonic acid metabolic pathway. In order to identify new genetic variants involved in NIUA we carried out a GWAS in a Spanish population. Method: Patients and controls were recruited in clinical centres integrated in the Spanish Network for Allergic Diseases. All patients experienced more than two episodes with at least two different unrelated NSAIDs, in the absence of any other concomitant chronic disease. Genome-wide scans were conducted using Axiom Genome-Wide CEU Array chips, for 308 NIUA patients and 144 NSAIDs tolerant age and sex-matched controls.

Results: A total of 32 SNPs showed suggestive significant associations $\left(10^{-8}<P<10^{-5}\right)$ with NIUA. The lowest $P$ values corresponded to polymorphisms located on chromosome 17: rs7225428 in GOSR2 $\quad\left(P=1.11 \times 10^{-6}\right), \quad$ rs197111 $\left(P=7.68 \times 10^{-6}\right)$, and rs1071682 in $E F$ TUD2 $\left(P=1.77 \times 10^{-5}\right)$.

Conclusion: The identification of new variants potentially associated with NIUA provides us with new clues for understanding the underlying mechanisms of this pathology, the most important clinical entity induced by HRs.
61

Next generation sequencing of the $\lg E$ repertoire reveals high identity with other memory compartments

Patil, $\mathrm{S}^{1}$; Tadigotla, $\mathrm{VR}^{2}$; Shreffler, $\mathrm{W}^{1}$

Allergy and Immunology, Massachusetts General Hospital, Harvard Medical School, Boston, MA, United States: ${ }^{2}$ Courtagen, Woburn, MA, United States

Background: Mucosal immunoglobulins, $\mathrm{IgE}$ and $\mathrm{IgA}$, have an important role in allergic diseases and better understanding the development and renewal of human memory IgE and IgA will likely have therapeutic implications. In this study, we sought to test the hypothesis advanced by others that $\operatorname{IgE}$ is uniquely produced via continuous isotype switching from non-IgE memory populations by comparing the IgE and $\operatorname{IgA}$ repertoire by deep sequencing.

Method: Circulating memory B (CD19+ CD27+) cells were immunomagnetically isolated from the peripheral mononuclear cells of a healthy donor. Isolated RNA was PCR amplified using a mixture of constant and $\mathrm{V}$ region primers. Purified DNA was sequenced using Illumina MiSeq $2 \times 250$, including multiple sample controls. Overlapping reads were merged after adapter trimming using SeqPrep. IMGT HighV-Quest was used to generate VDJ mappings on merged reads. Statistical analysis was conducted using R. Related clones were identified as those transcripts sharing the same V, J, and CDR3 amino acid sequence.

Results: A total of 150176 individual BCR transcripts were identified. Of the 18099 IgA transcripts, 1669 related clones were identified. Of the $544 \mathrm{IgE}$ transcripts, 410 related clones were identified. Clonal diversity within the $\operatorname{IgE}$ sequences was significantly greater than the IgA diversity $(P<0.001)$.

Of the $379 \operatorname{Ig}$ A related clones that were shared between both $\operatorname{IgM}$ and $\operatorname{IgG}$, only $2.4 \%$ of the related transcripts shared $100 \%$ identity to either IgM or IgG transcripts. In contrast, of the $97 \mathrm{IgE}$ related clones that were shared between both IgM and $\mathrm{IgG}$ transcripts, $90.2 \%$ transcripts were identical with either IgM or IgG transcripts $(P<0.001)$. Of the 113 IgE transcripts that were clonally related to either $\operatorname{IgM}(10)$ or $\operatorname{IgG}$ (103), the IgG-related IgE transcripts had significantly longer CDR3 length (17.2 vs $14.2, P=0.02)$ and a higher number of non-silent mutations (7.65 vs 3.00, $\quad P<0.001)$, suggesting increased somatic hypermutation of those IgGrelated sequences.

Conclusion: Human IgE memory cells are rare, demonstrate greater repertoire diversity but substantially closer sequence homology to the $\operatorname{IgG}$ repertoire than do memory IgA cells. Distinctly different from $\operatorname{IgA}$, these findings support the paradigm that human $\operatorname{IgE}$ memory cells are short lived and predominantly derived from switching of long-lived IgG memory cells.

\section{2}

Sequence characterisation of peanut allergen-specific human $B$ cell repertoires

Hoh, RA ${ }^{1}$; Joshi, $\mathrm{SA}^{1}$; Looney, $\mathrm{TJ}^{1}$; Roskin, $\mathrm{KM}^{1}$; $\overline{\text { Glanville, }} \mathrm{J}^{1}$; King, JK ${ }^{1}$; Wang, $\mathrm{C}^{1}$; Pham, TD ${ }^{1}$; Liu, $\mathrm{Y}^{1}$ Jackson, $\mathrm{KK}^{1}$; Lee, J-Y'; Dixit, VP'; Rivetta, $\mathrm{CV}^{1}$; Lyu, $\mathrm{S}^{-C^{2}}$; Nadeau, $\mathrm{KC}^{2}$; Boyd, $\mathrm{SD}^{1}$

${ }^{1}$ Pathology, Stanford University School of Medicine, Stanford, CA, United States; ${ }^{2}$ Pediatrics, Stanford University School of Medicine, Stanford, CA, United States

Background: The B cells of peanut allergic patients produce allergen-specific IgE antibodies, but the molecular features of these antibodies are largely unknown, and the changes induced in allergen-specific B cell clones by oral immunotherapy (OIT) are unclear.

Method: We have developed a novel flow cytometry protocol for sorting single peanut allergen-specific B cells from cryopreserved PBMC samples using fluorescently labeled peanut allergens Ara h 1 and Ara h 2.

Results: Analysis of peanut-specific B cells from 30 allergic patient samples taken at baseline and during OIT, and from three healthy controls, reveals an increase in the median frequency of allergen-specific memory B cells observed from baseline to time points taken during OIT. Monoclonal antibodies generated from paired immunoglobulin heavy and light chains from FACS-sorted peanut allergen-specific single $\mathrm{B}$ cells show specific binding to Ara $\mathrm{h} 1$ or Ara $\mathrm{h} 2$ in ELISA and Western blotting assays, and epitope mapping demonstrates binding to known major epitope regions in these proteins. Of 68 well-characterised 
peanut-specific antibodies from seven patients with peanut allergy, approximately $30 \%$ were expressed as various $\mathrm{IgG}$ isotypes, and the remaining peanut-specific antibodies were of $\operatorname{IgA}$ and $\operatorname{IgM}$ isotypes. Peanut-specific IgG4 sequences were observed only in subjects undergoing OIT. Peanut-specific IgM antibodies were highly mutated relative to overall IgM repertoires. Conclusion: We have isolated peanut-specific peripheral blood $\mathrm{B}$ cells expressing antibodies that bind allergen proteins Ara h 1 and Ara h 2, and determined their sequence characteristics, isotype expression, and clonal lineage features. We are comparing these data with high-throughput DNA sequencing (HTS) datasets of overall B cell repertoires in the same patients, to extend our understanding of the clonal lineages of allergen-specific B cells and their alterations in response to OIT.

\section{3}

Prenatal factors affecting FOXP3 DNA methylation in umbilical cord blood Tregs and mononuclear cells

North, $\mathrm{ML}^{1,2}$; Omana, $\mathrm{V}^{3}$; Macisaac, ${ }^{4}$; Mah, $\mathrm{S}^{4}$; Day, $\mathrm{A}^{5} ;$ Brook, $\mathrm{J}^{6} ;$ Kobor, $\mathrm{M}^{4,7}$; Ellis, $\mathrm{AK}^{1,2}$

${ }^{1}$ Departments of Medicine and Biomedical \& Molecular Science, Queen's University, Kingston, ON, Canada;

${ }^{2}$ Allergy Research Unit, Kingston General Hospital, Kingston, ON, Canada; ${ }^{3}$ Biomedical and Molecular Sciences, Queen's University, Kingston, ON, Canada; ${ }^{4}$ Centre for Molecular Medicine \& Therapeutics, Child \& Family Research Institute, Vancouver, BC, Canada; ${ }^{5}$ Kingston General Hospital, Clinical Research Centre, Kingston, ON, Canada; ${ }^{6}$ Environment Canada, Toronto, ON, Canada; ${ }^{7}$ University of British Columbia,

Vancouver, BC, Canada

Background: Regulatory $\mathrm{T}$ Cells (Tregs) are important immunomodulatory cells thought to influence the development of allergy and asthma. The Forkhead Box Protein 3 (FOXP3) transcription factor is essential for Treg function and is regulated by DNA methylation in the proximal promoter region. Methylation in the Treg-specific demethylated region (TSDR) is indicative of the frequency of Tregs in mononuclear cells (MNCs). We hypothesized that maternal allergy, prenatal smoking and mode of delivery would have effects on FOXP3 DNA methylation in umbilical cord blood.

Method: Cord blood samples were collected at the time of delivery from 86 women who gave informed consent. Allergy was defined as a positive self-report of allergy verified by a clinically relevant positive skin prick test. Smoking was defined as exposure to tobacco smoke more than 3 days per week for more than half the pregnancy. CD4 + CD127lowCD49d- cells were isolated using magnetic sorting techniques, validated by flow cytometry, and DNA was bisulfite converted. DNA methylation at the FOXP3 proximal promoter and TSDR were assessed by pyrosequencing in the same participants in both mononuclear cells (MNCs) and Tregs. Multivariate analysis of variance was employed to explore the effects of prenatal factors on DNA methylation. The covariates of sex, gestational age, mode of delivery, maternal smoking and maternal allergy were tested for independently, while controlling for all other covariates.

Results: 28 women were confirmed allergic, 27 smoked during pregnancy, and 43 delivered by cesarean. As FOXP3 is on the $\mathrm{X}$ chromosome, sex of the child significantly affected DNA methylation $(P<0.0001)$. In Tregs, FOXP3 promoter methylation was significantly affected by maternal smoking $(P=0.039)$, and maternal allergy $(P=0.007)$. DNA methylation at the TSDR in cord blood MNCs was significantly affected by mode of delivery $(P=0.026)$.

Conclusion: In this birth cohort, we demonstrate an effect of mode of delivery on overall Treg numbers. Maternal allergy and prenatal smoking affected DNA methylation of the FOXP3 promoter within isolated Treg cells.

\section{4 \\ The search for candidate genetic variants regulating the $\mathrm{IgE}$ response to parasites and allergens}

Acevedo, $\mathrm{N}^{1,2}$; Bornacelly, $\mathrm{A}^{1}$; Mercado, $\mathrm{D}^{1}$; Unneberg,

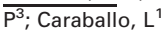

${ }^{1}$ Institute for Immunological Research, Cartagena, Colombia; ${ }^{2}$ Translational Immunology Unit, Department of Medicine Solna, Karolinska Institutet, Stockholm, Sweden; ${ }^{3}$ Science for Life Laboratory, Stockholm, Sweden

Introduction: The burden of common and rare genetic variants may regulate traits such as total and specific IgE levels. In addition to exome variants those in promoters, untranslated regions and introns might predispose to high/low IgE response phenotypes. Our previous findings suggest that specific antibody levels to the nematode Ascaris lumbricoides are under genetic control by chromosome 13. The aim of this study was to explore other regions and select candidate markers for population genotyping.

Methods: Coding and non-coding regions of 14 genes including CHI3L1, CHIA, IL10, FCER1A, RAD50/IL13, IL4, IL5, TSLP, IL33, STAT6 and the Ascaris-infection susceptibility locus 13q33 (LIG4, $A B H D 13, \quad T N F S F 13 B, \quad I R S 2)$ were sequenced using targeted enrichment (SureSelect, Agilent) and massive parallel sequencing (Illumina HiSeq2000). We included 48 subjects (24 asthmatics/24 controls) from Cartagena, Colombia at the extreme of the distribution $(<25$ th and $>75$ th percentiles) of total and specific $\operatorname{IgE} /$ IgG levels to Ascaris; variant discovery and genotyping were done using GATK. Burden analyses comparing extreme groups were done using Ingenuity Variant Analysis.

Results: Twenty one variants observed in high IgE responders to Ascaris (at least four subjects) were not found in individuals with $\operatorname{IgE}$ levels below the 25 th percentile. These variants were distributed in STAT6, CHIA, CHI3L1, IL13, LIG4 and $A B H D 13$. Subjects with high specific IgE levels to Ascaris had a significant enrichment of six variants in the promoter and $5^{\prime} \mathrm{UTR}$ of STAT6 (burden $P$ value $=0.009$ ) and seven coding variants in CHIA $(P=0.04)$. Enrichment of STAT6 variants was also found in subjects with high total IgE levels $(P=0.04)$ and high $\operatorname{IgG}$ levels to Ascaris $(P=0.005)$. Two IgE-related STAT6 variants were also overrepresented in asthmatics but the correlation between $\operatorname{IgE}$ and asthma enriched variants was low. Asthmatic patients had a significant enrichment of variants in the TSLP gene $(P=0.02)$.

Conclusion: Using deep sequencing we have discovered genetic variants overrepresented in high IgE producers. The burden of non-coding variants in STAT6 might influence the strength of $\operatorname{IgE}$ response. Coding variants with predicted deleterious effects on chitinase genes were associated to high $\operatorname{IgE}$ responses. The relationship between enriched variants and the $\operatorname{IgE} / \operatorname{IgG}$ responses to Ascaris and environmental allergens will be evaluated in two independent case-control datasets.

\section{5 \\ Innate IgG molecules and innate B cells, identified serologically by immunoglobulin constant heavy $\mathbf{G}$ chain $(/ G H G)(F c g)(G M)$ genes, are involved in IgE sensitisation and allergy phenotypes}

Oxelius, V-A ${ }^{1,2}$

Department of Pediatrics and Institute of Laboratory Medicine, Lund University, Lund, Sweden; ${ }^{2}$ Department of Clinical Immunology, Lund University, Lund, Sweden

The distinguished alternative GM allotypes localised in immunoglobulin constant heavy $\mathrm{G}$ chains, IGHG (Fcg) (GM) genes on chromosome 14q32.3 define two unique variants of respectively $\operatorname{IgG} 3, \operatorname{IgG} 1$ and IgG2 subclass, with different structures and functions. Fixed combinations of g3, g1 and g2 allotypes constitute the haplotypes, 
which are indirect markers of four B cells. The $I G H G$ allele (allotypes), expressed in homozygous (restricted IgG molecules) or heterozygous forms, are assessed by new serological methods. The GWAS and HapMap do not include $I G H G$ genes. These common Mendelian IGHG genes, respond differently to allergens, bacterial and viral infections, vaccination and passive immunotherapy. $I G H G$ genes have impact on diseases as allergy, immunodefi- ciency, autoimmunity and malignancy. Association/linkage of different $I G H G$ genes give information about risk/protection, good or bad prognosis, for improvement of clinical care. Increased $\operatorname{IgE}$ or $\operatorname{IgE}$ sensitisation are mainly found in the $I G H G^{*} b f n$ haplotype (innate $\mathrm{B}^{* \mathrm{bfn}}$ or $\mathrm{B} 1$ cell) with increased innate $\operatorname{IgG} 2 * \mathrm{n}$ levels. The $I G H G$ gene map of healthy Caucasians is given.
References: V.-A. Oxelius, J.P. Pandey, Human immunoglobulin constant heavy $G$ chain $(I G H G)(\mathrm{Fc} g)(\mathrm{GM})$ genes, defining innate variants of $\mathrm{IgG}$ molecules and $\mathrm{B}$ cells, have impact on disease and therapy, Clinical Immunology (2013) 149, 475-486.

V.-A. Oxelius, From genotypes of immunoglobulin constant heavy $G$ chains $(I G H G)(\mathrm{Fc}$ g) (GM) genes to phenotypes in childhood asthma, Int. Arch.Allergy Immunol. (2012) 159 94-102. 


\section{Oral Abstract Session}

\section{OAS 12 - Novel vaccines for AIT}

\section{6 \\ Immunogenicity evaluation of subcutaneous administration of peptide hydrolysate from Lolium perenne (gpASIT+TM) in combination with bacterial HSP70 (DnaK) in patients with seasonal allergic rhinitis}

Shamji, $\mathrm{MH}^{1}$; Ceuppens, $\mathrm{J}^{2}$; Hellings, $\mathrm{P}^{3}$; Durham $\mathrm{SR}^{1}$ Duchateau, $\mathrm{J}^{4}$; Legon, $\mathrm{T}^{4}$; Pirotton, $\mathrm{S}^{4}$

${ }^{1}$ Imperial College London-NHLI, Royal Brompton Hospital, Allergy and Clinical Immunology, London, United Kingdom; ${ }^{2}$ Department of Allergy and Clinical Immunology, Biotech Tools sa, UZ Leuven, Leuven, Belgium; ${ }^{3}$ ENT Department, Biotech Tools sa, UZ Leuven, Leuven, Belgium; ${ }^{4}$ Biotech Tools sa, Brussels, Belgium

Background: We have developed a peptide hydrolysate from Lolium perenne (gpASIT+TM) for the treatment of grass polleninduced allergy that can be administered either orally or subcutaneously. Bacterial HSP70 (DnaK) can reduce inflammation and can be used as an adjuvant. The aim of the study was to assess immunogenicity of gpASIT $+\mathrm{TM}$ in the presence or absence of DnaK.

Method: In a phase IIa, double-blind, randomised, single-centre trial study, participants were treated with gpASIT+TM/ DnaK $(n=12)$ or gpASIT alone $(n=12)$. Subjects received five subcutaneous injections of increasing doses of gpASIT $+\mathrm{TM}$ or a combination of gpASIT+TM and an equal amount of DnaK over 4 weeks. Grass pollen-specific serum IgG4 and blocking antibody responses were evaluated by ImmunoCAP and IgE-FAB assay, respectively.

Results: Although symptoms and rescue medication scores were not reduced in the gpASIT/DnaK group compared to the gpASIT alone group, grass pollen-specific IgG4 levels increased following treatment (week 7) in both groups (gpASIT group: $P<0.001 ; \quad$ gpASIT/DnaK group: $P=0.003)$. Grass pollen-specific IgE levels did not increase following treatment in either group, although the levels transiently increased during the pollen season (gpASIT group: $P=0.011 ; \quad$ gpASIT/DnaK group: $P=0.014$. Grass pollen-specific IgG4 levels were increased at week 7 and were decreased during the pollen season. Both treatments induced $\mathrm{IgG}$-associated blocking antibodies (week 7; $P<0.001$ in both groups). Contrary to the effect on
IgG4, the effect on blocking antibodies persisted about 1 year after discontinuation of treatment (week 52, gpASIT group: $P<0.001 ; \quad$ gpASIT/DnaK group: $P=0.007)$. Neither treatment elicited specific IgA levels.

Conclusion: For the first time, we report that subcutaneous administration of peptide hydrolysate from Lolium Perenne is associated with long-term induction functional $\operatorname{IgG}$ antibodies responses. Large studies are required to evaluate the clinical effects of gpASIT with/without DnaK.

67

AllerTтм: safety and efficacy of a birch pollen allergy vaccine based on contiguous overlapping peptides in a phase Illb study

Jutel, $\mathrm{M}^{1,2}$; De Blay, $\mathrm{F}^{3}$; Jacobsen, $\mathrm{L}^{4}$; Spertini, $\mathrm{F}^{5}$. $\overline{\text { Worm, }} \mathrm{M}^{6}$; Majorek-Olechowska, $\mathrm{B}^{7}$; Della Corte, $\mathrm{G}^{8}$; Reymond, $\mathrm{C}^{8}$

Department of Clinical Immunology, Wroclaw Medica University, Wroclaw, Poland; ${ }^{2}$ ALL-MED' Medical Research Institute, Wroclaw, Poland; ${ }^{3} \mathrm{CHRU}$

Strasbourg, Strasbourg, France; ${ }^{4}$ ALC Copenhagen, Copenhagen, Denmark; ${ }^{5}$ Division of Immunology and Allergy, CHUV, Lausanne, Switzerland; ${ }^{6}$ Charite Universitätsmedizin Berlin, Berlin, Germany; ${ }^{7}$ AlergoMed Specjalistyczna Przychodnia Lekarska, Tarnow, Poland; ${ }^{8}$ Anergis SA, Epalinges, Switzerland

Background: Reduction in duration of treatment and number of injections, as well as standardisation of extracts and safety, represent a medical need in subcutaneous immunotherapy (SCIT). AllerTтм (Anergis SA, Switzerland), a mixture of three contiguous overlapping peptides (COPs) derived from the major birch pollen allergen, Bet v1, and administered according to an ultra-fast regimen, has been evaluated for efficacy and safety in a placebo-controlled, double-blind, randomized, multicenter, European phase IIb trial in patients with moderate to severe birch pollen allergic rhinoconjunctivitis.

Method: 239 patients, included into three arms (placebo, $50 \mu \mathrm{g}$ and $100 \mu \mathrm{g}$ COPs in Aluminum hydroxide), received five preseasonal subcutaneous injections within 2 months. Efficacy was evaluated during the subsequent 2013 birch pollen season with a combined Rhinoconjunctivitis Symptom and Medication Score (RSMS) as primary endpoint and Quality of Life (mini-RQLQ) as secondary endpoint.

Results: RSMS (median of differences) was reduced by $30 \%$ with AllerT $50 \mu \mathrm{g}$ (Wilcoxon: $P=0.024$ ) compared to placebo and by $19 \%$ with AllerT $100 \mu \mathrm{g}$ $(P=0.190)$.

AllerT improved Mini-RQLQ also by $30 \%$ compared to placebo at $50 \mu \mathrm{g}$ dose (Wilcoxon: $P=0.048$ ) and by $26 \%$ at $100 \mu \mathrm{g}$ dose $(P=0.069)$. AllerT was safe and well tolerated. Most AEs were mild to moderate, the most frequent being local reactions at the injection site. Only four (two in each actively treated group) grade 3 systemic reactions (WAO grading) were recorded out of a total of 769 injections of active product, all occurring more than 30 min after injection.

Conclusion: Ultra-fast immunotherapy with AllerTтм at $50 \mu \mathrm{g}$ dose significantly improves symptoms and medication score and Quality of Life in birch pollen rhinoconjunctivitis with a favorable safety profile compared to conventional SCIT.

\section{8}

Four doses of Der $p$ derived synthetic peptide immuno-regulatory epitopes over 3 months results in a persistent treatment effect at 1 year on symptoms of House Dust Mite allergy

Hafner, RP ${ }^{1}$; Couroux, $\mathrm{P}^{2}$; Armstrong, $\mathrm{K}^{3}$; Salapatek, $\overline{\mathrm{AM}^{4} ; \text { Patel, }} \mathrm{D}^{2}$; Larche, $\mathrm{M}^{5}$

${ }^{1}$ Circassia Ltd, Oxford, United Kingdom; ${ }^{2}$ Topstone Research, Mississauga, ON, Canada; ${ }^{3}$ Adiga Life

Sciences, Hamilton, ON, Canada; ${ }^{4}$ Inflamax Research, Mississauga, ON, Canada; ${ }^{5}$ Department of Medicine, McMaster University, Hamilton, ON, Canada

Background: Treatment with seven Synthetic Peptide Immuno-Regulatory Epitopes (SPIREs) derived from Fel d1 improved symptoms of cat allergy one year after four injections 4 weeks (wk) apart (Patel JACI 2013). In this study we evaluated the effect of treatment with a mixture of seven synthetic $\mathrm{T}$ cell epitopes derived from Dermatophagoides pteronyssinus (Der p) on symptoms of House Dust Mite (HDM) allergy.

Methods: Subjects with a history of allergic rhinoconjunctivitis to HDM attended a baseline challenge of three consecutive days of 4-h allergen exposures (Der p 20- 
$120 \mathrm{ng} / \mathrm{m}^{3}$ ) in an Environmental Exposure Chamber (EEC). Subjects scored 4 nasal and four non-nasal symptoms on a scale of 0-3 every $30 \mathrm{~min}$ and these were summed to give a Total Rhinoconjunctivitis Symptom Score (TRSS) (0-24). Subjects reattended the EEC at $19 \mathrm{wk}, 36 \mathrm{wk}$ and $49-50$ wk. In phase 1 subjects were randomised to $11 \times 3 \mathrm{nmol}, \quad 11 \times 6 \mathrm{nmol}$, $11 \times 12 \mathrm{nmol}$ HDM SPIRE or placebo $(N=20$ per group). Following a preplanned interim analysis after the $19 \mathrm{wk}$ EEC visit, subject numbers were expanded in phase 2 to compare $4 \times 12 \mathrm{nmol}$ vs $11 \times 12 \mathrm{nmol}$ vs placebo $(N=44$ per group). After the 49-50 wk EEC subjects completed a global assessment of change.

Results: The interim analysis at $19 \mathrm{wk}$ showed a mean change in TRSS of -3.97 with $12 \mathrm{nmol}$ HDM SPIRE vs -1.83 on placebo. At the 49-50 week EEC visit $4 \times 12$ nmol HDM SPIRE showed a mean change in TRSS of -5.82 vs -3.04 on placebo $(P<0.05)$. The global assessment of change correlated with improvement in TRSS, with $68 \%$ of subjects receiving $4 \times 12$ nmol HDM-SPIRE scoring 'Any Better' vs $44 \%$ on placebo $(P=0.05)$. Treatment with $11 \times 12 \mathrm{nmol}$ did not result in incremental improvement even in more symptomatic subjects.

Conclusions: A short course of HDMSPIRE administered as four injections of a $12 \mathrm{nmol}$ dose over $12 \mathrm{wk}$ showed a substantial improvement 50 wk after the start of treatment in subjects' HDM allergy symptoms in an EEC model using both symptom scores and global assessment of change.

\section{9}

A short course of synthetic peptide immuno-regulatory epitopes derived from grass allergens leads to a reduction in grass allergy symptoms

Ellis, $\mathrm{AK}^{1,2}$; Frankish, $\mathrm{CW}^{1,3}$; Armstrong, $\mathrm{K}^{4}$; Larche, $\mathrm{M}^{5}$; O'Hehir, RE ${ }^{6}$; Steacey, LM ${ }^{1}$; Hafner, RP

${ }^{1}$ Allergy Research Unit, Kingston General Hospital, Kingston, ON, Canada; ${ }^{2}$ Department of Medicine, Queen's University, Kingston, ON, Canada; ${ }^{3}$ Kanata Allergy Services, Ottawa, ON, Canada; ${ }^{4}$ Adiga Life Sciences, Hamilton, ON, Canada; ${ }^{5}$ Department of Medicine, McMaster University, Hamilton, ON, Canada; ${ }^{6}$ Department of Allergy, Immunology \& Respiratory Medicine, The Alfred Hospital \& Monash University Melbourne, Vic., Australia; ${ }^{7}$ Circassia Ltd, Oxford, United Kingdom

Background: Synthetic Peptide ImmunoRegulatory Epitopes (SPIREs) derived from Fel dl and Amb al improve symptoms of cat allergy (Patel et al. JACI 2013) and ragweed allergy (Hafner et al. JACI 2011). In this study we evaluated whether treatment with a mixture of seven $\mathrm{T}$ cell epitopes derived from Rye, Bermuda and
Timothy Grass allergens (Grass-SPIRE) can reduce grass allergy symptoms in an Environmental Exposure Unit (EEU).

Methods: Subjects attended the EEU approximately 4-5 months before the start of the grass pollen season for four consecutive days of $3 \mathrm{~h}$ exposures to rye grass pollen (target $3500 \pm 500$ grains $/ \mathrm{m}^{3}$ ). Subjects scored 4 nasal and four non-nasal symptoms on a scale of $0-3$ every $30 \mathrm{~min}$ and these were summed to give a Total Rhinoconjunctivitis Symptom Score (TRSS) (024). 282 subjects were randomised to placebo or one of three Grass-SPIRE regimens. After the peak of the grass pollen season, subjects re-attended the EEU approx. 25 weeks (wk) after randomisation. At the end of the final EEU visit subjects completed a seven point global assessment of symptomatology changes compared to the baseline EEU visit.

Results: $8 \times 6 \mathrm{nmol}$ Grass-SPIRE $2 \mathrm{wk}$ apart showed a mean change in TRSS after the grass pollen season of -5.4 vs -3.8 on placebo $(P<0.05)$ in the protocol specified primary endpoint. $8 \times 6 \mathrm{nmol}$ Grass SPIRE showed a mean change in Total Nasal Symptom Score of -2.3 vs -1.6 on placebo $(P<0.05) .8 \times 6 \mathrm{nmol}$ Grass-SPIRE showed a mean change in Total Non-Nasal Symptom Score of -3.1 vs -2.2 on placebo $(P=0.08) .44 \%$ of subjects treated with the $8 \times 6$ nmol regimen considered themselves very much better or much better compared to the baseline EEU visit vs $22 \%$ of subjects on placebo $(P<0.01)$.

Conclusions: Treatment with $8 \times 6 \mathrm{nmol}$ Grass-SPIRE 2 wk apart improved symptoms of grass allergy in an EEU model. The change in symptoms included changes in both nasal and non-nasal symptoms. The improvement in symptom scores correlated with an improvement in the global assessment of change.

\section{0 \\ Intralymphatic immunotherapy induces allergen specific plasma cells; an open pilot study \\ Nezam, $\mathrm{H}^{1}$; Schmid, $\mathrm{JM}^{1,2}$; Madsen, $\mathrm{HHT}^{2,3}$; Schmitz

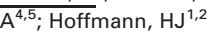 \\ ${ }^{1}$ Department of Respiratory Diseases, Aarhus University Hospital, Aarhus, Denmark; ${ }^{2}$ Department of Clinical Medicine, Aarhus University, Aarhus, Denmark; ${ }^{3}$ Department of Radiology, Aarhus University Hospital, Aarhus, Denmark; ${ }^{4}$ Department of Hematology, Aalborg University Hospital, Aalborg, Denmark; ${ }^{5}$ Aalborg University Hospital, Aalborg Hospital Science and Innovation Center, Aalborg, Denmark}

Background: Allergen specific immunotherapy is currently the only treatment that modifies the underlying mechanism of allergic diseases and limits type-I allergic reactions. We evaluated the effect of
Intralymphatic immunotherapy (ILIT) in an open pilot study on patients with allergic rhinoconjunctivitis due to grass pollen. We counted allergen-specific plasma cells 1 week after injection and measured basophil sensitivity (BAT EC50) as biomarkers predicting clinical effect.

Method: Seven participants with a history of symptomatic allergic rhinoconjunctivitis due to grass pollen allergy, whose symptoms had been monitored for 3 years, were treated with three ultrasound guided allergen injections in an inguinal lymph node at $28 \pm 3$ days' intervals before the grass pollen season. Peripheral blood plasma cells were measured as Trucount-standardised $\mathrm{CD} 45^{+} \mathrm{CD} 19^{+} \mathrm{CD} 20^{-} \mathrm{CD} 27^{+} \mathrm{CD} 38^{+} \mathrm{IgE}^{+}$ ${ }^{-}$Grass $^{+}$cells $/ \mathrm{ml}$ before treatment, and 7 days after each injection visit. Basophil sensitivity was measured as EC50 for CD63 on CD193+ basophils at each injection visit, and after the ensuing grass pollen season. Clinical outcome was assessed as symptom medication score (SMS) with standardised rescue medication and rhinoconjunctivis quality of life questionnaires (RQLQ) during the grass pollen seasons.

Results: Side effects were recorded after three of 21 injections: one participant each registered local swelling at the injection site, local itching and a grade I reduction in peak flow. Plasma cell frequency increased 7 days after the second and third injection visits ( $P=0.016$ each). There was no effect on basophil sensitivity. The combined SMS decreased significantly after ILIT $(P=0.0313)$. The maximum increase in allergen specific plasma cell concentratin correlated with reduction in symptom medication score $(r=0.96, n=5)$.

Conclusion: ILIT appears to be well tolerated. The maximal number of allergen specific plasma cells after 1 week after ILIT predicts protection to symptoms in the ensuing season. 


\section{Oral Abstract Session}

\section{OAS 13 - From mechanisms to diagnosis of rhinitis}

\section{1 \\ Diagnostic evaluation of hypersensitivity reactions to betalactam antibiotics in a large population of children}

Torres, $\mathrm{MJ}^{1}$; Zambonino, $\mathrm{MA}^{1}$; Corzo, $\mathrm{JL}^{2}$; Muñoz, $\mathrm{C}^{2}$; $\overline{\text { Requena, }}^{2}$; Ariza, $A^{3}$; Mayorga, $C^{3} ;$ Urda, $A^{2}$; Blanca, $\mathrm{M}^{1}$

${ }^{1}$ Allergy Unit, IBIMA, Regional University Hospital of Malaga, UMA, Malaga, Spain; ${ }^{2}$ Carlos Haya Hospital, Paediatric Service, Malaga, Spain; ${ }^{3}$ IBIMA, Regional University Hospital of Malaga, UMA, Research

Laboratory, Malaga, Spain

Background: Hypersensitivity reactions to betalactams (BLs) are often reported in children, with amoxicillin and, to a lesser extent, cephalosporins being the drugs most frequently involved. Although many of these children are initially considered to be allergic, a careful evaluation only confirms a low percentage. The aim of the study was to analyse the clinical data, sensitisation profile and diagnostic methods used in a large group of children with a clinical history of hypersensitivity reactions to BLs.

Method: Children aged 1-14 years with symptoms suggestive of hypersensitivity to BLs from January 2006 to December 2012. Diagnosis was confirmed by means of clinical history, specific IgE determination, skin testing and, if necessary, a drug provocation test (DPT).

Results: Out of 783 patients studied, only $62(7.9 \%)$ were confirmed as being allergic, $9(14.5 \%)$ with immediate and $53(85.5 \%)$ with non-immediate reactions. In those children with immediate reactions, 2 $(22.2 \%)$ were diagnosed by in vitro test, 2 $(22.2 \%)$ by skin testing and $5(55.6 \%)$ by DPT; in those with non-immediate reactions, $2(3.8 \%)$ were diagnosed by skin testing and $51(96.2 \%)$ by DPT. DPT was positive to the culprit drug (29 AX-CLV, $26 \mathrm{AX}$, one cefixime and one cefaclor) in all cases, and the most common symptoms were exanthema (43 cases), urticaria in 12, urticaria-angioedema in one, and erythema in one case.

Conclusion: Over $90 \%$ of the children evaluated were finally confirmed as tolerant to BLs. Most reactions were of the nonimmediate type, and DPT was an essential tool for diagnosis.
72

Reactions to iodinated contrast media in an allergy unit

Jara Gutiérrez, P; Rondon Cepeda, H; Valverde Monge, M; Fernandez Nieto, M; Sastre Dominguez, J Allergy, Fundacion Jimenez Diaz, Madrid, Spain

Background: The interventional and diagnostic procedures using iodinated radio contrast media (RCM) are being performed with increasing frequency over the last years. Although the rate of side-effects from iodinated-RCM is low, there are an increasing number of patients who experience reactions after the administration of a RCM.

Hypersensitivity reactions to iodinated radio contrast media (RCM) are either immediate-type (IT) or delayed reactions (DT).

Method: A retrospective analysis of fiftythree patients with a history of reaction to RCM during 2012 (January-December) were performed.

Skin prick tests and intradermal reaction (IR) with RCM (Iodixanol, Iohexol, Iopamidol and/or Iomeprol) with dilutions at $1 / 10,1 / 100$ were carried out. Single-blind placebo-controlled intravenous drug challenge test (DCT) was carried out in those cases with a negative skin tests.

Results: 37 female and 16 male, with a mean age of $56.27( \pm 16.02)$ reported the following reactions: cutaneous reactions (33), angioedema (8) systemic reactions (7) or inespecific reactions (5). These reactions were identified as IT in $24(45 \%)$ and DT in $29(55 \%)$ patients.

In 24 subjects with a history of IT, 1 $(4.1 \%)$ was skin-test (IR) positive to Iodixanol and Iohexol but negative to iopamidol with good tolerance by DCT to iopamidol, DCT was positive in two cases $(8.3 \%)$ to Iodixanol in spite of negative skin tests.

In 29 subjects with a history of DT, 2 $(6.8 \%)$ were skin-test (IR) positive: 1 to Iodixanol and the other to Iodixanol, Iohexol and Iopamidol. DCT was positive in seven cases $(24.13 \%)$ to Iodixanol in spite of negative skin tests.

Of the nine patients with DCT positive despite of negative skin test, five presented the reaction 6 months before the study, two between 6-12 months before and two more than 1 year before.
Conclusion: Skin tests (IR) allowed to identify allergy to RCM in $5.6 \%$ of our patients, while intravenous challenge test in $15.9 \%$ of patients. The sensitivity of skin tests was low, demonstrating the need to perform DCT for a correct diagnosis.

73

Low reproducibility and lack of histamine release in positive intracutaneous tests to penicillin and insulin

Tannert, LK; Falkencrone, S; Moertz, CG; Skov, PS; Bindslev-Jensen, C

Department of Dermatology and Allergy Centre, Odense Research Centre for Anaphylaxis (ORCA), Odense University Hospital, Odense C, Denmark

Background: In contrast to patients sensitised to e.g. grass, peanut and insulin where more than $90 \%$ will have both positive intracutaneous (intradermal) test (ICT) and IgE, only approximately $1 / 3$ of patients with a positive ICT to penicillin also have positive $\operatorname{IgE}$ and vice versa. The mechanism behind this is unknown.

Aim: To investigate real time histamine release in wheals elicited by penicillin, insulin and grass.

Method: 18 patients with previous positive immediate ICT to penicillin, seven with positive non-immediate ICT to penicillin, six with positive immediate ICT to insulin, six grass pollen allergics and 13 controls were included. Skin microdialysis was performed as described by Jelstrup-Petersen (1998). Histamine in the eluate was measured at baseline and after ICT $(50 \mu \mathrm{l})$ with culprit or non-culprit drug/allergen, whereafter residual histamine from skin mast cells was released by injection of codeine. Histamine in the eluate was subsequently analyzed by flourescense spectroscopy (Skov et al. 1985).

Results: Reproducibility of previous positive immediate ICT with penicillin was low (three of 18), but high for non-immediate reactions (seven of seven), whereas three of six previous positive immediate reactions to ICT with insulin could be reproduced. Histamine was present in the eluate from only one of the positive immediate ICT (three penicillin, three insulin), whereas all grass allergics released histamine upon ICT with grass. Five patients were subsequently challenged with penicillin, two with a positive outcome. These two also developed 
specific IgE and a positive immediate reaction to penicillin. A third developed specific IgE to penicillin only.

Conclusion: Reproducibility over time of ICT (immediate reactions) with penicillin and insulin is low. Interesting, not all positive reactions are accompanied by a measurable histamine release determined by in situ microdialysis. The validity of immediate reactions in ICT to penicillin and insulin must be interpreted with caution.

\section{4}

Anti-infliximab antibodies production and clinical consequences: adverse reactions and loss of response

Matucci, $\mathrm{A}^{1}$; Petroni, $\mathrm{G}^{2}$; Nencini, $\mathrm{F}^{2}$; Pratesi, $\mathrm{S}^{2}$; Maggi, $\mathrm{E}^{2}$; Vultaggio, $\mathrm{A}^{3}$

${ }^{1}$ Immunoallergology Department, Careggi Hospital, Florence, Italy; ${ }^{2}$ Department of Experimental and Clinical Medicine, Transfer and High Education DENOTHE, University of Florence, Florence, Italy; ${ }^{3}$ Immunoallergology Department, Centro Hospitalar São João, EPE, PortoUnit, Careggi Hospital, Florence, Italy

Background: Infliximab (IFX), a chimeric anti-TNF $\alpha$ monoclonal antibody, is an established targeted therapy for immunomediated inflammatory diseases. As other biotherapeutics, it carries potential risk of immunogenicity, with the production of specific anti-IFX antibodies (ATI), leading to potential loss of response or acute infusion reactions. This study was aimed to analyse the development of ATI in IFXtreated patients.

Methods: A panel of 216 patients suffering from Rheumatoid Arthritis, Spondyloarthritis, Vasculites and Bowel Inflammatory Diseases treated with IFX were enrolled. They were categorized into five groups, including responder (I group), non responder [totally (II) or partially (III)], reactive (IV) and reactive/non responder (V) patients. Non isotype-specific ATI were measured by a double-capture Elisa kit assay (Immundiagnostik) and titred through sera dilutions.

Results: Decreased percentages of positivity were shown in different groups of patient: $100 \%$ in $\mathrm{V}, 75 \%$ in IV, $41.5 \%$ in III, $16.7 \%$ II and $11.5 \%$ in the group of responders. When evaluated for their titres, the reactive patients (IV plus $\mathrm{V}$ groups) exhibited the highest amounts of ATI, usually being three time more then those exhibited by other groups. A longitudinal study of more than 30 patients showed that the development of ATI occurred after the initial infusions of IFX in all groups of patients, even after the interruption of therapy. In the group of patients who interrupted IFX for adverse infusion reactions or unresponsiveness we evaluated the kinetics of ATI in the absence of therapy or in the presence of alternative regimens (other anti-TNF $\alpha$ or anti-IL-6R mAbs).

Conclusions: The direct correlation between the titre of ATI and the adverse drug reactions, as well as their onset during the first infusions of different cycles of therapy are relevant issues for prevention and management of such patients.

This study was supported by IMI project ABIRISK:

\section{5}

Functional eicosanoid testing correlation to systemic ASA challenge

Schäfer, D ${ }^{1} ;$ Maier, $\mathrm{H}^{2}$; Mühlmeier, $\mathrm{G}^{2}$

Medical Clinic III FAU Erlangen-Nuremberg, Erlangen, Germany; ${ }^{2}$ Bundeswehrzentralkrankenhaus, Ulm, Germany

Background: Systemic acetylsalicylic acid (ASA) challenge is an established method confirming the diagnosis of NSAID-triggered hypersensitivity like aspirin exacerbated respiratory diseases (AERD). The functional eicosanoid testing (FET) is part of the diagnostic process, which can be performed even in outpatient departments. The correct diagnosis is required for an appropriate treatment Therefore the correlation of FET and in vivo ASA challenge in patients suffering from NSAID-triggered hypersensitivity was investigated.

Method: Fifty-two patients (32 female, 28 male, 28-74 years), clinically characterised by number of polypectomy, duration of symptoms of rhinitis and/or asthma, were examined. Peripheral venous blood for the FET was collected before performing the systemic ASA challenge.

Results: ASA challenge was positive in $67 \%$, negative in $21 \%$, and could not be performed in $12 \%$ patients due to uncontrolled asthma.

The mean FET values ranged from 1.3 to 1.6 and 2.3 for patients with negative to positive ASA-challenge, and challenge test positive patients with recurrent polyposis and asthma, respectively. The FET values correlated best with results of ASA-challenge test and symptoms of medical history of all patients investigated.

Conclusion: The FET reflects some of the known complex pathogenic mechanisms of NSAID-triggered hypersensitivity. The FET results correlated with those of the systemic ASA challenge test. The ASA challenge test confirmed not all diseased patients. Therefore, our results endorsed the idea, that the FET approach might support the diagnostic decision process even in outpatients without the risk of in vivo ASA challenge test, and therefore might support the appropriate treatment of patients suffering from NSAID-triggered hypersensitivity.
76

Aspirin downregulates FOSL1 disrupting pulmonary protection in aspirin induced asthma

Kacprzak, D ${ }^{1}$; Wieczfińska, J1; Pospiech, $\mathrm{K}^{2}$; Bednarek, $A^{2}$; Nowakowska, $M^{2}$; Pniewska, $E^{1}$; Pawliczak, $R^{1}$ ${ }^{1}$ Department of Immunopathology, Medical University of Lodz, Łódź, Poland; ${ }^{2}$ Department of Molecular Cancerogenesis, Medical University of Lodz, Łódź, Poland

Background: Up to twenty percent of adult asthmatics with nasal polyps are sensitive to aspirin and no unambiguous theory exists which provides a satisfactory explanation for the occurrence of aspirininduced asthma (AIA) in some asthmatic patients.

Aim: The main aim of this study was to recognise possible gene targets involved in the pathogenesis of aspirin-induced asthma by comparing the AIA genetic profile against ATA and healthy controls in peripheral blood mononuclear cells (PBMCs) in Polish population by means of whole genome scan after aspirin challenge.

Methods: Peripheral blood mononuclear cells (PBMCs) were separated from blood of three groups of subjects - aspirin sensitive asthmatics (ASA), aspirin tolerant asthmatics (ATA) and healthy volunteers (HV) and then stimulated by either lysine aspirin or lysine as a endogenous control. Next, RNA was isolated, transcribed into cDNA and subjected to microarray (six ASA, three ATA and four HV) and RTPCR (11 ASA, seven ATA and 15 HV) studies. Simultaneously, protein was extracted from PBMCs and used in further Western Blot analysis (six ASA, six ATA and seven HV).

Results: Microarray studies have revealed that mRNA expression of FOSL1 in PBMCs of AIA was significantly lower than in HV ( $t$-test, $P=0.04)$. This result was confirmed on the protein level ( $t$-test, $P=0.001$ ). Expression of mRNA FOSL1 in AIA studied using RT-PCR was not significantly lower in comparison to $\mathrm{HV}$ (Mann-Whitney test, $P=0.091$ ). FOSL1 mRNA expression analyzed by RT-PCR was significantly lower in PBMCs of AIA vs ATA ( $t$ - test, $P=0.031$ ), what also was confirmed by results of Western Blot method ( $t$-test, $P=0.001$ ).

Conclusion: Demonstrated significant decrease in expression of FOSL1 at either mRNA or protein level in patients diagnosed with AIA in comparison to ATA and controls may constitute the novel insight into the pathogenesis of AIA.

Research funded by Polpharma Scientific Foundation no 6/VII/2008 and by The Polish National Science Centre no 2011/01/N/ NZ5/00334. 


\section{Oral Abstract Session}

\section{OAS 14 - Novel mechanisms of immune regulation}

\section{7}

In vitro human Th2 differentiation: IL-4 is essential for IL-9 and IL-31 induction and not IL-5 and IL-13 production

Blom, L; Poulsen, LK

$\overline{\text { Allergy }}$ Clinic, Copenhagen University Hospital

Gentofte, Hellerup, Denmark

Background: It is well described that Th2 cell differentiation in vivo and in vitro is induced by IL-4 that induces GATA-3 and secretion of the hallmark Th2 cytokines IL-4, IL-5 and IL-13. However, it has been proposed that Th2 cell differentiation might occur through a default endogenous program independently of IL-4. The aim of this investigation was to study the importance of IL-4 in human Th2 cell differentiation with primary focus on production of the Th2 cell cytokines IL-5, IL-9, IL-10, IL-13 and IL-31.

Method: Naïve human $\mathrm{CD}^{+}$

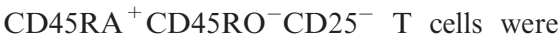
stimulated with fibroblast- bound antiCD3/CD28 with and without IL-2, IL-4, anti-IFN- $\gamma$ and anti-IL-4, for up to 15 days following flow cytometry and multiplex analysis of secreted cytokines.

Results: Interestingly, neutralisation of IL4 resulted in significantly higher frequency of IL-13+ CD4 + T cells with a tendency of more IL-4+ and IL-5+ CD4+ T cells when compared to the Th2 cultures, with added IL-4. Moreover, even though the IL-5 secretion was slightly lower in the cultures with anti-IL-4, both IL-5 and IL-13 secretion were strongly induced independently of IL-4. In contrast to the Th2 cell type cytokines IL-5 and IL-13; IL-9 and IL-31 secretion were only observed in cultures with exogenously added and/or endogenously produced IL-4. Inhibition of IFN- $\gamma$ and T-bet expression was highly dependent on addition of IL-4 and not neutralisation of IFN- $\gamma$. Moreover, GATA-3 expression was higher in the cultures with added IL-4. Lastly, all cultures had an increased release of IL-10 over the stimulation period, with significantly higher levels in Th2 cultures especially in the early priming phase.

Conclusion: Taken together, we show that secretion of IL-9 and IL-31 as well as repression of IFN- $\gamma$ and T-bet expression critically depends on IL-4. In contrast, the
Th2 hallmark cytokines IL-5 and IL-13 were induced independently of IL-4.

\section{8}

Interleukin-35 suppresses the activation of memory/effector Blo t 5-specific murine Th2 cells

Huang, C-H; Loo, EX-L; Soh, GH; Chua, LH; Wen,

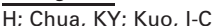

Department of Paediatrics, National University of Singapore, Singapore City, Singapore

Background: The regulatory $\mathrm{T}$ cells are one of the crucial mechanisms in maintaining the immune homeostasis. Several distinct groups of regulatory and suppressive $\mathrm{T}$ cells are the main mediators in preventing autoimmune diseases and control the chronic inflammatory diseases such as inflammatory bowel diseases and asthma. Regulatory $\mathrm{T}$ cells associated cytokines transforming growth factor- $\beta$ (TGF- $\beta$ ) and IL-10 are major contributors to the suppressive functions of regulatory $\mathrm{T}$ cells. However, results derived from extensive studies on the immune suppressive functions of TGF- $\beta$, IL-10 and regulatory T cells under normal and immune-pathological conditions have shown that the bioactivity and functions of these 'inhibitory cytokines' are highly pleiotropic in vivo. Systematic administration of such therapeutic cytokines could be undesirable because of its potential pleiotropic activities that may lead to toxicities in vivo. The regulatory $\mathrm{T}$ cells associated $\mathrm{IL}-35$ has potent suppressive function in vitro and in mouse disease models.

Method: To examine whether IL-35 could act directly on effector Th2 cells, we expressed IL-35 from HEK293 cells and tested the effect on a Blo t 5-specific memory/effector Th2 cell line. The supernatant collected from PCIneo-IL-35 transfected HEK293 cells was co-cultured in various doses with the Blo $t$ 5-specific memory/ effector Th2 cells.

Results: Recombinant IL-35 exhibited a dose dependent suppressive effect on the proliferation of effector Th2 cells in the $\left[{ }^{3} \mathrm{H}\right]$-thymidine incorporation assay. The transcript for I112rb2 was up-regulated in activated Blo t 5-specific memory/effector
Th2 cells but the mRNA expression of gp130 remained unchanged.

Conclusion: Our results indicated that IL35 , in addition to induce IL-35-producing induced regulatory $\mathrm{T}$ cells from naïve cells, may act directly on Th2 memory/effector cells. The information is important in designing application strategies for IL-35 in future immunotherapies.

\section{9}

The association of increased serum IL-35 levels with cholesterol concentrations in patients with coronary artery disease

Chalubinski, M; Gorzelak, P; Wojdan, K; Luczak, E; Duraj, I; Mozdzan, M; Broncel, M Department of Internal Diseases and Clinical Pharmacology, Laboratory of Tissue Immunopharmacology, Medical University of Lodz, Lodz, Poland

Background: Atherosclerosis leading to coronary artery disease (CAD) is a chronic inflammatory condition, in which plenty of cytokines are involved. However, the role of recently discovered immunosuppressive IL-35 in CAD development and its relation with CAD risk factors (cholesterol levels and postmenopausal status) are unknown. The aim of the study was to assess concentrations of IL-35 in sera of CAD patients and healthy subjects and to analyze the association between IL-35 and total cholesterol, LDL and HDL cholesterol levels, and left ventricular ejection fraction (LVEF).

Method: IL-35 was assessed by ELISA in 31 patients with CAD confirmed by coronary angiography and 30 healthy controls. Additionally, total cholesterol, LDL and HDL cholesterol, and triglycerides levels were assessed. LVEF was analyzed by echocardiography.

Results: CAD patients had higher concentrations of IL-35 as compared to healthy controls $\quad(58.1 \pm 16.6 \mathrm{pg} / \mathrm{ml}$ vs $5.35 \pm$ $3.35 \mathrm{pg} / \mathrm{ml} ; P<0.001)$. IL-35 levels negatively correlated with total cholesterol $(R=-0.43, P<0.0001)$ and LDL cholesterol $(R=-0.31, P<0.01)$ levels and positively correlated with HDL cholesterol in men $(R=0.53, P=0.001)$. In women, IL35 levels negatively correlated with LVEF $(R=-0.48, P=0.01)$ and positively with 
the duration of postmenopausal status $(R=0.57, P=0.001)$.

Conclusion: IL-35 may be involved in atherogenesis and CAD. IL-35 levels may be associated with CAD risk factors - total cholesterol, LDL and HDL cholesterol levels and postmenopausal status.

\section{0 \\ Synergy between TLR-2 and TLR-3 signalling in primary human nasal epithelial cells}

van Tongeren, J; Golebski, K; van Egmond, D; de Groot, E; Fokkens, W; van Drunen, CM Otorhinolaryngology, University of Amsterdam Academic Medical Center, Amsterdam,

The Netherlands

Introduction: Although we have a detailed understanding of how single microbial derived triggers activate specialised TLRs on airway epithelial cells, we know little of how these receptors react in a more complex environment. As in everyday life nasal epithelial cells are exposed to multiple TLR agonists, we wanted to explore whether exposure to one trigger could affect the responsiveness to another TLR trigger.

Methods: Primary nasal epithelium from healthy individuals and the bronchial epithelium cell line NCI-H292 were exposed to different TLR agonists and the effect on the expression of different TLRs was determined using the quantitative PCR. Moreover, we evaluated the effect of TLR-3 stimulation on TLR-2 functionally by ELISA.

Results: Stimulation of airway epithelium with a specific TLR agonist affects gene expression of another TLRs. In primary nasal epithelium, poly(I:C) challenge results in an up-regulation of the TLRI, $T L R 2$, and TLR3 genes and reduction of expression of TLR5. Furthermore, we show that poly(I:C) induced activation of TLR-2 contributes to stronger cell responses to the TLR-2 agonist PGN and that regulation of these synergistic responses may take place at the mRNA level of IL6 and IL8. Although the effect of TLR-3 stimulation on TLR-2 functionality and most of the effects on the expression of other TLRs could be replicated in our NCI-H292 model, poly(I:C) failed to up-regulate TLRI and showed an additional up-regulation of TLR4.

Conclusion: Our data suggests that to better understand TLR mediated innate responses we need to consider the impact of the presence of multiple triggers.

81

Interleukin 1 beta induces stem cell factor production by epithelial cells via a hypoxia-inducible factor one pathway: new insights into the crosstalk between inflammation and allergy

Wyszynski, RW ${ }^{1}$; Varani, L ${ }^{2}$; Gibbs, BF ${ }^{1}$; Sumbayev, $\mathrm{VV}^{1}$ Medway School of Pharmacy, University of Kent, Chatham Maritime, United Kingdom; ${ }^{2}$ Institute for Research in Biomedicine, Bellinzona, Switzerland

Background: Stem cell factor (SCF) is a hematopoietic growth factor that controls the differentiation of myeloid cells including allergic effector cells such as mast cells. Mast cells require continuous exposure to SCF for their development and function. $\mathrm{SCF}$ is produced by endothelial/epithelial cells and fibroblasts, cells which express receptors for interleukin-1 $\beta$ (IL-1 $\beta$ ), a major pro-inflammatory cytokine. Our aims were to address whether IL-1 $\beta$ plays a major role in inducing SCF production from epithelial cells and determine the intracellular mechanisms that control SCF generation triggered by this inflammatory cytokine.

Methods: MCF-7 epithelial cells were stimulated with IL- $1 \beta$ and SCF production as well as hypoxia-inducible factor-1 (HIF-1) activation were analysed by Western blot, ELISA and quantitative real-time PCR. IL-1 $\beta$ and SCF levels in the blood plasma of healthy human donors were assayed by ELISA.

Results: We observed that IL-1 $\beta$ induced SCF expression and production by MCF-7 cells, a process which was directly dependent on HIF-1 activation. Experiments with pharmacological inhibitors revealed that IL-1 $\beta$-induced HIF-1 activation was dependent on PI-3 kinase/mTOR pathway. Furthermore, we also observed a positive correlation between SCF and IL-1 $\beta$ levels in the blood plasma of healthy human donors.

Conclusions: Our studies demonstrated that IL-1 $\beta$ induces the expression and production of SCF by human epithelial cells in a HIF-1-dependent manner. These findings confirm the cross-talk between innate immune defences and allergic responses suggesting an ability of IL- $1 \beta$ to potentiate mast cell development and function through SCF production.
82

Mast cells derived exosomes induces mesenchymal phenotype in lung epithelial cell line

Shelke, GV; Lässer, C; Lötvall, J

Krefting Research Centre, The Sahlgrenska Academy, University of Gothenburg, Medicine, Gothenburg, Sweden

Background: Airway diseases are associated with disturbed epithelial repair, secreted extracellular matrix and fibrosis. Many studies on lung alveolar epithelial cells have indicated that these cells are involved in development of deformed lung niche, but how they get activated is still illusive. Mast cells surrounding the lung epithelium can communicate to neighboring cell via many secreted factors including exosomes, extracellular membrane vesicles releases by cells that can transfer proteins and RNA molecules between cells. In this study we determined whether alteration of lung epithelia is mediated through mast cells derived exosomes.

Method: Exosomes were isolated from conditioned media from human mast cells (HMC1). Uptake of HMC1 derived exosomes (PKH-67 dye) by A549 cells was detected by florescent microscopy. Morphology analysis and inverse migration assay were performed to evaluate the migratory phenotype in A549 after receiving $\mathrm{HMCl}$ exosome. Finally, expression for $\mathrm{N}$-cadherin and $\mathrm{E}$-cadherin was done performed to validate epithelial to mesenchymal transition (EMT) phenotype. Oxidative stress protection assay was also performed by treating A549 cells with HMC1 exosomes cells prior to H202 treatment.

Results: $\mathrm{HMCl}$ derived exosome were taken up by A549 cells and localised preferential to one end of the nuclear periphery. Lung epithelial cells showed fibroblast like morphology and migratory phenotype when exposed to mast cells derived exosomes. This phenotype was related to enhanced surface expression of N-Cadherin and reduces expression of E-Cadherin. Also upon oxidative stress, lung epithelia undergo cell cycle arrest which was rescued by the treatment of mast cells exosomes.

Conclusion: Mast cell derived exosomes protect against oxidative stress and induce an EMT like phenotype in lung epithelial cells. This could explain the development of disintegrate lung epithelial phenotypes. 


\section{Oral Abstract Session}

\section{OAS 15 - Pro- and anti-allergic impact of infections}

\begin{abstract}
83
Associations between helminth infections and atopy and allergy-related

diseases in island communities in Uganda: baseline findings of the Lake Victoria Island intervention study on worms and allergy-related diseases (LaVIISWA)

Webb, E ${ }^{1} ;$ Nampijja, $\mathrm{M}^{2}$; Nerima, $\mathrm{B}^{2}$; Elliott, $\mathrm{A}^{1,2}$ 'London School of Hygiene and Tropical Medicine, London, United Kingdom; ${ }^{2}$ MRC/UVRI Uganda Research Unit on AIDS, Uganda Virus Research Institute, Entebbe, Uganda
\end{abstract}

Background: Immune responses to helminths and allergens are related, but chronic helminth infections may suppress effector responses in the host. Effective helminth control may result in increases of allergyrelated disease. Our objective was to examine associations between helminths and allergy-related conditions at baseline in a cluster randomised trial of intensive vs standard anthelminthic intervention in heavily exposed communities.

Methods: A household survey was conducted among 26 fishing villages in the Koome islands, Lake Victoria, Uganda. Asthma was assessed by oral and videoquestionnaire, atopic dermatitis by inspection, atopy by skin prick testing (mites and cockroach). Helminth infections were ascertained by stool, urine and haemoparasitology. Associations between helminths and allergy-related conditions were examined using multivariable logistic regression, adjusting for the survey design.

Results: 2395 people took part. Adjusting for the survey design, prevalence of wheeze was $4 \%$ by oral and $8 \%$ by video-questionnaire; prevalence of atopy was $19 \%$; prevalence of visible flexural dermatitis was $<1 \%$. $73 \%$ of individuals had S. mansoni detected by urine assay and $57 \%$ by stool Kato Katz examination; $10 \%$ had Trichuris. In multivariable regression models allowing for the survey design, S. mansoni and Trichuris were positively associated with atopy (adjusted odds ratios [95\% confidence interval] $1.38[1.03,1.83]$ and 2.03 $[1.35,3.07])$, with the positive association dominated by the response to cockroach. There was a weak positive association with wheeze in under-fives for S. mansoni $(2.77$ $[0.38,20.31])$ and Trichuris (3.51 [0.70,
17.65]) and in over-five-year-olds for Ascaris $(4.59$ [1.13, 18.64]).

Conclusion: We found positive associations between helminths and allergy-related outcomes. However, the prevalence of SPT positivity for mites (but not cockroach), and of wheeze and eczema, was lower among island children than among children in the lake shore town of Entebbe (data not shown), suggesting that the island environment is, overall, protective against allergy-related disease. Immunological investigations are being undertaken to explore mechanisms behind our results.

84

Early immune response patterns to pathogenic bacteria are associated to increased risk of lower respiratory infections in children

Vissing, $\mathrm{NH}^{1}$; Larsen, $\mathrm{JM}^{2}$; Rasmussen, $\mathrm{MA}^{3}$; Thysen $\mathrm{AH}^{1,2} ;$ Chawes, $\mathrm{BL}^{1}$; Bønnelykke, $\mathrm{K}^{1}$; Brix, $\mathrm{S}^{2}$; Bisgaard, $\mathrm{H}^{1}$

${ }^{1}$ Copenhagen Prospective Studies on Asthma in Childhood, Health Sciences, University of Copenhagen \& Danish Pediatric Asthma Center, Copenhagen University Hospital, Gentofte, Denmark; ${ }^{2}$ Department of Systems Biology, Technical University of Denmark, Lyngby, Denmark; ${ }^{3}$ Department of Food Science, University of Copenhagen, Frederiksberg, Denmark

Background: Neonatal colonisation of the airways with respiratory pathogens is associated with increased risk of lower respiratory infections (LRI) in early childhood (1). Therefore, we hypothesized that children developing LRI have an abnormal immune response to pathogenic bacteria in infancy. We aimed to characterise the systemic immune response to pathogenic bacteria at the age of 6 months and study the association with incidence of LRI during the first 3 years of life.

Method: The Copenhagen Prospective Study on Asthma in Childhood 2000 (COP$\mathrm{SAC}_{2000}$ ) is a prospective clinical study of a birth cohort of 411 children born of mothers with asthma. The cohort was followed closely with 6-monthly planned visits and visits at acute respiratory episodes during the first 3 years of life. The cytokine immune response (TNFa, IFNg, IL-17, IL5, IL-13, IL-10, IL-2) to $H$. influenzae, $M$. catarrhalis and $S$. pneumoniae was analyzed in 292 infants using peripheral blood mononuclear cells isolated and stored since the age of 6 month. Data were analyzed by poison regression towards incidence of LRI in combination with principal component analysis (PCA). Analyses were adjusted for covariates associated to LRI and stratified for asthmatic disease within the first 3 years of life.

Results: Cytokine levels from the three different bacterial stimulations exhibited a particular pattern identifying children at risk of LRI independently of asthma $\left(P_{a d j}<0.001\right)$. The pattern was influenced by several cytokines and differential responses to different bacteria rather than production of one specific cytokine.

Conclusion: Children at risk of LRI present a distinct immune-fingerprint upon exposure to common airway pathogens. Reference: 1. Vissing NH, Chawes BLK, Bisgaard H. Increased risk of pneumonia and bronchiolitis after bacterial colonisation of the airways as neonates. $\mathrm{Am} \mathrm{J}$ Respir Crit Care Med 2013;188:1246-1252.

\section{5}

Primary nasal epithelial cell responses to HRVA1B infection of subjects with or without atopic rhinitis and asthma

Taka, $\mathrm{S}^{1}$; Megremis, $\mathrm{S}^{1}$; Spyridaki, IS ${ }^{1}$; Skevaki, $\mathrm{CL}^{1}$; Trochoutsou, $\mathrm{Al}^{1}$; Roumpedaki, $\mathrm{E}^{1}$; Emmanouil, $\mathrm{P}^{2}$; Kontogianni, $\mathrm{K}^{2}$; Bakakos, $\mathrm{P}^{2}$; Loukides, $\mathrm{S}^{3}$; Passioti, $\mathrm{M}^{1}$; Kokkinou, $\mathrm{D}^{1}$; Apostolopoulou, $\mathrm{M}^{1}$; Papadopoulos, $\mathrm{NG}^{1,4}$

${ }^{1}$ Allergy Department, 2nd Pediatric Clinic, National and Kapodistrian University of Athens, Athens, Greece; ${ }^{2} 1$ st Respiratory Medicine Department, National and Kapodistrian University of Athens, Athens, Greece; ${ }^{3}$ 2nd Respiratory Medicine Department, National and Kapodistrian University of Athens, Athens, Greece; ${ }^{4}$ Centre for Paediatrics and Child Health, University of Manchester/Institute of Human Development,

Manchester, United Kingdom

Background: Defective type I interferon (IFN) production and consequent increased viral load have been described in bronchial epithelium of atopic asthmatic patients. The aim of the present study was to evaluate rhinovirus (RV) mediated IFN$\beta$ expression and RV load in nasal epithelial cells (NEC) of individuals with or without allergic rhinitis and asthma.

Method: NECs derived from 18 donors: six healthy non-atopic controls, six with allergic rhinitis without asthma and six with allergic rhinitis and asthma. Antiviral responses (IFN- $\beta$ mRNA, IFN- $\lambda$ protein), 
viral replication, cytotoxicity and inflammation (CCL5, IL-6) were assessed.

Results: Antiviral responses were deficient in NECs of asthma patients: levels of IFN$\beta$ mRNA, as well as IFN- $\lambda$ protein, were significantly lower in this group at all timepoints in comparison with both the allergic rhinitis and control groups $(P<0.05)$. Unexpectedly, NECs from allergic rhinitis patients expressed significant higher levels of IFN- $\beta$ mRNA. Viral replication and cytotoxicity were higher in NECs from both asthmatics and rhinitics, than those of controls at $48 \mathrm{~h}$.

Inflammation was also more pronounced in cells from asthmatics, as levels of CCL5 and IL6 were significantly higher in this group at $48 \mathrm{~h}$.

Conclusion: IFN- $\beta$ deficiency and the associated increased RV replication and cytotoxicity, appear to be present in the upper, in addition to the lower, respiratory epithelium in patients with allergic asthma. Although downstream effects (increased viral replication and cytotoxicity) are also present in allergic rhinitis NECs, this is associated with increased IFN levels, suggesting a reactive mechanism which is under evaluation.

\section{6 \\ Role of neutrophils in visceral leishmaniasis in India \\ Sharma, $\mathrm{S}^{1}$; Davis, $\mathrm{RE}^{2}$; Nylen, $\mathrm{S}^{3}$; Wilson, $\mathrm{ME}^{2}$. Sundar, $\mathrm{S}^{4}$ \\ ${ }^{1}$ Department of Medicine, Institute of Medical Sciences, Institute of Medical Sciences, Varanasi, India; \\ ${ }^{2}$ University of lowa and The VA Medical Centre, lowa City, IA, United States; ${ }^{3}$ Department of Microbiology Tumor and Cell Biology, Karolinska Institutet, Solna, Sweden; ${ }^{4}$ Department of Medicine, Institute of Medical Sciences, Banaras Hindu University, Varanasi, India}

Background: Visceral leishmaniasis (VL), also know as Kala-azar, is a disease caused by Leishmania donovani in India. In this form of leishmaniasis the parasite spreads to visceral organs, and disease is fatal if left untreated. Isolated PBMCs from patients with VL lack antigen specific immune responses. Whether they play regulatory roles has yet to be unveiled. We found that PBMCs isolated from VL patients contained a distinct subset of low density granulocytes staining for neutrophil markers. Surprisingly, these cells stained for MHC II expression, and MHC II staining was more intense in cells from $\mathrm{VL}$ patients than cells from Endemic Healthy Controls (EHC).

Method: Flow Cytometry was performed using BD Facs Calibur and analysed using FlowJO software (Treestar).

Gene Expression: Human neutrophils were isolated from whole blood after RBC lysis using Militenyi CD66abce microbead kit to get highly pure neutrophils (>97\%). Results: Neutrophils from patients with VL were examined for activation. A minimal increase CD11b high CD62L low neutrophils was observed in whole blood of VL patients than in EHC. Total blood neutrophils from patients with VL contain a subset of HLA DR expressing cells that stain as neutrophils. Low density granulocytes that co-purified with PBMCs contained a population expressing $\mathrm{MHC}$ II and CD66b (neutrophil marker). These cells were more abundant in VL patients than EHC. PMN in the RBC pellet separated by migration through the ficoll layer behave as 'classical neutrophils' and do not show appreciable MHC II expression in both VL patients and Endemic healthy groups.Differences were observed in expression of cytokine and chemokine mRNAs in the same subjects during active VL and after treatment.

Conclusion: We observed a unique population of neutrophils like cells expressing MHC II in chronic VL. We need to determine if these cells are supressive as reported by Abebe et al. or function in antigen presentation.

\section{7}

High degree of overlap between responses to a virus and to the house dust mite allergen in airway epithelial cells

Golebski, K; van Egmond, D; de Groot, E; Röschmann, KIL; Fokkens, WJ; van Drunen, CM

Otorhinolaryngology, University of Amsterdam -

Academic Medical Center, Amsterdam, The Netherlands

Background: Airway epithelium is widely considered to play an active role in immune responses through its ability to detect changes in the environment and to generate a microenvironment for immune competent cells. Therefore, besides its role as a physical barrier, epithelium affects the outcome of the immune response by the production of various pro-inflammatory mediators.

Methods: We stimulated airway epithelial cells with a viral analogue poly(I:C) or with the house dust mite allergen in a $24-\mathrm{h}$ time course. The gene expression pattern was determined by Real-Time PCR and cytokines production by multiplex ELISA. Results: We demonstrate that the temporal pattern of the genes that respond to virus exposure in airway epithelium resembles to a significant degree their pattern of response to HDM. The gene expression pattern of EGR1, DUSP1, FOSL1, JUN, $M Y C$, and $I L 6$ is rather similar after poly
(I:C) and HDM exposure. However, both triggers also induce a specific response (e.g. $A T F 3, F O S$, and NFKB1). We confirmed these data by showing that epithelial cells produce a variety of similar mediators in response to both poly(I:C) and HDM (IL1-RA, IL-17, IFN- $\alpha$, and MIP1- $\alpha$ ), sometimes with a quantitative difference in response (IL2-R, IL-6, IL-8, MCP-1, MIG, and HGF). Four mediators (IL-12, IP-10, RANTES, and VEGF) where up-regulated specifically by poly(I:C) and not by HDM. Additionally, pre-exposure to HDM deregulates production of cytokines and mediators in response to poly(I:C).

Conclusions: Epithelial cells responses to the HDM-allergen and a virus strongly resemble both in gene expression and in protein level explaining why these two responses may affect each other.

\section{8}

A single influenza virus infection protects of allergic airway inflammation in the OVA-mouse model

Hudemann, $\mathrm{C}^{1}$; Skevaki, $\mathrm{C}^{1}$; Matrosovich, $\mathrm{M}^{2}$; Renz, $\mathrm{H}^{1}$ Institute of Laboratory Medicine and

Pathobiochemistry, Molecular Diagnostics, Philipps Universität Marburg, Marburg, Germany; ${ }^{2}$ Institute of Virology, Philipps Universität Marburg, Marburg, Germany

Asthma and other chronic airway inflammatory diseases are heavily affected by airway viral infections. Epidemiological and clinical data display contradictory effects in terms of asthma prevention and asthma exacerbation in already diseased patients. The molecular basis of these phenotypemodifying virus-host interactions, however, is still largely unknown. The impact of viral infections on the development of chronic airway inflammation depends on (i) virus strain and frequency of viral infection as well as, (ii) on host immune status and localisation of infection.

Here, we describe a prevention model of influenza airway infection followed by succeeding ovalbumin based airway inflammation model in Balb/c mice. A single infection using the pandemic $\mathrm{HH} / 09 \mathrm{H} 1 \mathrm{~N} 1$ causes a quick influx and activation of CD4 and CD8 T-cell populations into the lung, peaking at day $8-12$ post infection combined with a pronounced TNF- $\alpha$, IFN- $\gamma$ and IL-6 secretion pattern. Virus-specific tetramerpositive cytotoxic $\mathrm{T}$ - and effector memory cells peak in the lung at day 12 followed by a migration into spleen. Even 60 days post infection, tetramer-positive cytotoxic T-cells were detected in both lung and spleen.

A subsequent OVA-sensitisation protocol starting from 12 days post infection followed by OVA challenge resulted in decreased severity of a range of allergic 
airway inflammation parameters (e.g. eosinophilia $P<0.05$, IL-5 and IL-13 $P<0.01$, goblet cell hyperplasia $P<0.05$ ) compared to the OVA-treated group without a preceding influenza infection. CD8 memory cell subpopulations displayed a characteristic redistribution pattern, and further in-vitro analysis points towards a protective role. The latter is based on cross-reactive sequence recognition leading to virus-induced dampening of subsequent allergic airway inflammation.

Delineation of the underlying molecular and cellular rearrangements during virus infection will lead to a better understanding of the development and regulation of chronic inflammation and should identify novel targets for prevention of asthma induction and exacerbation. 


\section{Oral Abstract Session}

\section{OAS 16 - Asthma mechanisms}

\section{9 \\ MUC5AC induction by cytoplasmic DNA in human bronchial epithelial cells \\ Takizawa, T; Aizawa, A; Solongo, O; Yagi, H; Nishida, $\overline{Y ; ~ K o y a m a, ~} \mathrm{H}$; Arakawa, $\mathrm{H}$ \\ Pediatrics, Gunma University Graduate School of Medicine, Maebashi, Japan}

Background: Production of mucin from bronchial epithelial cells arguably plays a pivotal role in inflammatory lung diseases such as asthma. We have previously found that double strand RNAs (polyI:C) and TGF- $\alpha$ synergistically induce MUC5AC expression in bronchial epithelial cells. We here report that introduction of exogenous DNAs into the cytoplasm efficiently stimulates MUC5AC transcription.

Method: Poly(dA:dT) was introduced into a human bronchial epithelial cell line, NCI-H292 by lipofection. MUC5AC mRNA was quantified with RT-qPCR. Knocking-down with RNAi and inhibitors for several molecules were used to study underlying molecular mechanisms.

Results: Introduction of poly(dA:dT) into the cytoplasm of NCI-H292 increased MUC5AC mRNA expression. Since IL-1 $\beta$ has been implicated in MUC5AC expression, we first checked with caspasel inhibition. Inhibition of caspease1, which is activated in inflammasomes to produce IL$1 \beta$, did not affect MUC5AC expression. In contrast, inhibition of NF- $\mathrm{NB}$ by a drug or RNAi against RelA markedly suppressed MUC5AC expression induced by poly(dA: dT). We have also knock-downed STING by RNAi, which is an adapter molecule located on a endoplasmic reticulum and has been shown to play important roles in cellular response to exogenous DNA. Suppression of STING caused a marked reduction of MUC5AC expression as well. Conclusion: These results demonstrate that STING-NF-kB pathway is involved in cytoplasmic DNA-induced mucin production in human bronchial epithelial cells.

90

The effect of CpGODN on the expression of TLR9, ARs and the release of IL-4, IL-10 in P815 mast cells

Li, M-R; Jiang, H-D; Wang, Q-Y; Zhang, H-L
The Second Affiliated Hospital \& Yuying Children's Hospital of Wenzhou Medical University, Wenzhou, China

Background: To observe the effect of CpGOND on the expression of TLR9 and ARs and the release of IL-4 and IL-10 on P815 mast cells.

Method: P815 cells were exposed to various concentrations of CpGODN $(0-1 \mu \mathrm{M})$ and NECA $\left(5^{\prime}-\mathrm{N}\right.$-Ethylcarboxamido adenosine) $\left(0-10^{-4} \mathrm{M}\right)$, TLR9 and ARs mRNA was detected by Real-time PCR, IL-4 and IL-10 in the supernatants were measured by ELISA. P815 cells were cultured for $24 \mathrm{~h}$ by dividing into four groups: control group(A), CpGODN -treated (B), NECAtreated (C), CpGODN and NECA -treated (D).

Results:

1 The expression of TLR9 mRNA in CpGODN-treated was significantly elevated than that in control group $(P<0.05)$. The expression of A2AR $\backslash$ A2BR mRNA in CpGODN-treated was significantly declined than that in control group $(P<0.01)$ in P815 cells. Of A3R mRNA was no significant different between in CpGODN-treated and in control group $(P>0.05)$.

2 There was no significant different of TLR9 mRNA between NECA-treated and the control group $(P>0.05)$. The expression of A2AR A2BR A3R mRNA in NECA-treated was significantly elevated than that in control group $(P<0.01)$.

3 The expression of TLR9 mRNA in CpGODN-treated and CpGODN/ NECA-treated was significantly higher than that in control group. The expression of $A 2 A R \backslash A 2 B R \backslash A 3 R$ mRNA in CpGODN/NECA-treated was significantly lower than that in NECA-treated group $(P<0.01)$.

4 The level of IL-10 in CpGODN-treated were higher than that in control group $(P<0.05)$. The level of $\mathrm{IL}-4$ in NECA-treated were higher than that in control group $(P<0.05)$. The level of IL-10 in NECA-treated were lower than that in control group $(P<0.05)$.

5 The level of IL-4 in NECA-treated and CpGODN/NECA-treated group was significantly higher than that in control group $(P<0.01)$. The level of IL-10 in
CpGODN-treated and CpGODN/ NECA-treated group was significantly higher than that in control group $(P<0.01)$.

Conclusion: P815 cells do express TLR9, A2AR, A2BR, A3R, but not A1R. CpGODN could up-regulate TLR9 mRNA expression, and down-regulate A2AR $\triangle A 2 B R$ mRNA expression, no effect on the expression of A3R mRNA in P815 cells. NECA could enhance the expression of $\mathrm{A} 2 \mathrm{AR} \backslash \mathrm{A} 2 \mathrm{BR} \backslash \mathrm{A} 3 \mathrm{R}$ mRNA, but no effect on the expression of TLR9 mRNA. CpGODN could raise the secretion of IL10 in $\mathrm{P} 815$ cells, no effect on the release of IL-4. NECA could reduce the secretion of IL-10 and increase the secretion of IL-4 in P815 cells.

\section{1}

MicroRNA-based identification of epithelial SEC14L3 loss in experimental and human paediatric asthma

Bartel, $\mathrm{S}^{1}$; Schulz, $\mathrm{N}^{1}$; Alessandrini, $\mathrm{F}^{2}$; Theis, $\mathrm{F}^{3}$; Eickel$\overline{\text { berg, } \mathrm{O}^{1}}$; Kicic, $\mathrm{A}^{4,5,6}$; Freishtat, $\mathrm{RJ}^{7}$; Krauss-Etschmann, $\mathrm{S}^{1}$

${ }^{1}$ Helmholtz Zentrum München, Comprehensive Pneumology Center Institute of Lung Biology and Disease, Helmholtz Zentrum München and University Hospital of the Ludwig Maximilians University (LMU), Member of the German Center for Lung Research (DZL), München, Germany; ${ }^{2}$ Helmholtz Zentrum Muenchen, Center of Allergy and Environment (ZAUM), Technische Universität and Helmholtz Zentrum München, Member of the German Center for Lung Research (DZL), Munich, Germany; ${ }^{3}$ Helmholtz Zentrum Muenchen, MIPS, Institute for Bioinformatics and System Biology, Munich, Germany: ${ }^{4}$ Department of Respiratory Medicine, Princess Margaret Hospital for Children, Perth, WA, Australia; ${ }^{5}$ School of Paediatrics and Child Health, The University of Western Australia, Nedlands, WA, Australia; ${ }^{6}$ Telethon Institute for Child Health Research, Centre for Child Health, The University of Western Australia, Nedlands, WA, Australia; ${ }^{7}$ Division of Emergency Medicine, Children's National Medical Center, Washington, DC, United States

Background: Asthma is the most common chronic disease in children and there is an unmet clinical need to identify novel treatment targets. As miRNAs can address key regulatory molecules within signalling pathways, they may be exploited to identify and prioritize novel targets involved in its pathogenesis. Thus, we sought to use differentially expressed miRNAs of a murine ovalbumin (OVA) model to identify 
novel gene networks of key relevance for asthma.

Methods: Dysregulated whole lung miRNAs were identified in mice with OVAinduced asthma by qualitative and quantitative microarrays. In silico predicted target mRNAs were validated in reporter assays and lung tissue at the mRNA and protein level and confirmed in a model of house dust mite (HDM) induced asthma and in human nasal epithelial cells from asthmatic children.

Results: We validated transcription factor cAMP-responsive element binding protein (CREB1) and its transcriptional co-activators (CRTC1-3) as targets of four of the top 10 up-regulated miRNAs in vitro and in vivo. Furthermore, a CREB1 transcriptional target SEC14-like 3 (SEC14L3) was down-regulated in both murine models as assessed by qRT-PCR, western blot and specific immunostaining. The decrease SEC14L3 protein expression coincided with goblet cell metaplasia. Of note, three miRNAs $(-17,-21$ and -144$)$ were highly increased in nasal cell brushings of children with allergic asthma compared to healthy controls which was paralleled by a decrease in SEC14L3 expression.

Conclusion: We identified alterations in miRNA expression and propose they might result in a SEC14L3 down-regulation via modulating CREB/CRTC-mediated transcription in murine experimental as well as in human paediatric asthma. SEC14L3 expression might be suitable to monitor the integrity of the respiratory epithelium.

\section{2 \\ Characterisation of miR570-3p expression and function in human airway epithelium and in asthmatic patients}

Stellato, $\mathrm{C}^{1,2}$; Roff, $\mathrm{AN}^{3}$; Craig, $\mathrm{TJ}^{3}$; August, $\mathrm{A}^{4}$; Ishmael, $\mathrm{FT}^{3,5}$

${ }^{1}$ Department of Medicine and Surgery, University of Salerno, Baronissi, Italy; ${ }^{2}$ Department of Allergy and Clinical Immunology, Johns Hopkins University School of Medicine, Baltimore, MD, United States; ${ }^{3}$ Division of Pulmonary, Allergy and Critical Care Medicine,

Department of Medicine, Pennsylvania State University M.S. Hershey Medical Center, Hershey, PA, United States; ${ }^{4}$ Department of Microbiology and Immunology, Cornell University, Ithaca, NY, United States;

${ }^{5}$ Department of Biochemistry and Molecolar Biology, Pennsylvania State University College of Medicine, Hershey, PA, United States

Background: Posttranscriptional regulation of cytokines, chemokines and growth factors by the action of microRNAs (miRNAs) and RNA-binding proteins (RBPs) on stability or translation of mature transcripts is emerging as a central means of regulating the inflammatory response.

Methods: In this study, we identified upregulation of miR-570-3p upon treatment with TNF- $\alpha$ (50 ng/ml for $4 \mathrm{~h})$ by real-time PCR and protein assays in a screen of miRNA expression of primary normal human bronchial epithelial cells (NHBE) and the human airway epithelial cell line A549.

Results: Levels of miR-570-3p were increased in NHBE by $2.6 \pm 0.6$ $(P=0.01)$ and A549 cells by $4.6 \pm 1.4$ $(P=0.0068)$. In silico analysis using five integrated algorithms (TargetScan, miRanda, PITA, MirTarget2, CoMeTa) identified as putative targets of miR-570-3p several cytokines, chemokines and regulatory factors involved in inflammatory responses, including $\mathrm{TNF} \alpha$ itself, IL-6, CCL2/MCP-1, CCL4/MIP1 $\beta$, CCL5/RANTES, CXCL8/IL-8, and the RBP HuR. Transient overexpression of miR570-3p in untreated A549 cells significantly increased targets' basal mRNA and protein levels, while cytokine upregulation in TNF $\alpha$-stimulated cells was differently affected by miR570-3p overexpression. HuR protein expression was repressed by mir-570-3p; this effect was not changed by TNF $\alpha$ and was dependent on the HuR $3^{\prime}$ untraslated region (UTR), as assessed by the inhibitory effect of miR-570-3p on luciferase expression in A549 cells transfected with an HuR 3'UTR-bearing reporter vector. However, HuR mRNA decay rate measured by Actinomycin $\mathrm{D}$ cell treatment was not affected by increased mir-570-3p levels. Lastly, expression of miR-570-3p was studied in subjects with mild, steroid-naive asthma and control subjects ( $n=10$ both groups) in exhaled breath condensates (EBC) and serum and found to be significantly lower $(P=0.026)$ in EBC, while no difference was observed in serum. A significant Pearson correlation between EBC levels of miR-570-3p and FEV1 was found for asthmatic patients $(r=-0.57, P=0.050)$, but not healthy controls $(r=0.17, P=0.311)$. Conclusion: Upregulation of miR-570-3p may mediate TNF $\alpha$ effect on airway epithelial gene regulation; levels of miR-570$3 p$ in EBC may reflect its yet partially identified contribution to mucosal inflammation in asthma.

\section{3 \\ Effect of salmeterol/fluticasone propionate combination therapy on methacholine- and adenosine $5^{\prime}$ - monophosphate-induced air trapping in smokers with asthma}

Aguilar, $\mathrm{D}^{1}$; Jang, $\mathrm{D}^{1}$; Prieto, $\mathrm{L}^{2}$; Palop, $\mathrm{J}^{3}$; Llusar, $\mathrm{R}^{1}$; Herrera, $\mathrm{S}^{3}$; Pérez, $\mathrm{C}^{1}$

'Hospital Universitario Doctor Peset, Servicio de Alergología, Valencia, Spain; ${ }^{2}$ Universidad de Valencia, Valencia, Spain; ${ }^{3}$ Hospital Universitario Doctor Peset, Servicio de Neumología, Valencia, Spain

Background: No information is available on the effect of ICS/LABA combination therapy on air trapping induced by inhaled bronchoconstrictor agents. The aim of the present study was to identify differences in the effect of salmeterol/fluticasone propionate $(\mathrm{SM} / \mathrm{FP})$ combination therapy on methacholine and adenosine 5'-monophosphate (AMP)-induced air trapping between smokers and nonsmokers with asthma.

Method: Methacholine- and AMP-induced changes in $\mathrm{FEV}_{1}$ and $\mathrm{FVC}$ were measured at baseline and after 6 weeks of treatment with $\mathrm{SM} / \mathrm{FP}(50 / 250 \mu \mathrm{g})$ twice a day in 22 current smokers and 22 non-smokers with asthma. The degree of air trapping induced by each bronchoconstrictor agent was expressed by the percent fall in FVC at a $20 \%$ fall in $\mathrm{FEV}_{1}$ relative to FVC after saline inhalation $(\triangle \mathrm{FVC} \%)$. Furthermore, patients completed a diary twice daily recording peak expiratory flow rate (PEFR), daytime and nighttime symptoms and use of rescue salbutamol.

Results: In nonsmokers with asthma, the mean $(95 \% \mathrm{CI}) \Delta \mathrm{FVC} \%$ values for AMP increased from $9.8 \%$ (7.9 to $11.8 \%)$ at baseline to $13.4 \% \quad(10.3$ to $16.6 \%$. $P=0.03)$ after 6 weeks of treatment with SM/FP. Furthermore, SM/FP conferred a significantly greater change in $\triangle \mathrm{FVC} \%$ values for AMP in those who did not smoke than in patients who smoked, the mean difference in the change between the groups being $4.4 \%(95 \% \mathrm{CI}, 0.5$ to $8.3 \%$, $P=0.03$ ). Treatment-induced increase in morning PEFR was significantly higher in nonsmokers than in smokers with asthma, the mean difference being $54.1 \mathrm{~L} / \mathrm{min}$ (95\% CI, 25.2 to $82.9 \mathrm{~L} / \mathrm{min}, P=0.0005$ ).

Conclusion: Although SM/FP inhalation therapy induces a greater improvement in pulmonary function in nonsmokers than in smokers with asthma, this treatment is associated with a paradoxical increase in AMP-induced air trapping only in nonsmokers. These observations might be relevant to understand the reported differences in the response to treatment between smokers and nonsmokers with asthma. 
94

A protective link between alveolar NO and remodelling processes in mild asthma?

Larsson-Callerfelt, A-K ${ }^{1}$; Weitoft, $\mathrm{M}^{1}$; Tufvesson, $\mathrm{E}^{2}$; Bjermer, $\mathrm{L}^{2}$; Westergren-Thorsson, $\mathrm{G}^{1}$

${ }^{1}$ Lung Biology, Lund University, Lund, Sweden;

${ }^{2}$ Respiratory Medicine and Allergology, Lund

University, Skåne University Hospital, Lund, Sweden

Background: High level of nitric oxide (NO) in the exhaled breath is as a marker for on going inflammation in the airways of asthmatic subjects. However, little is known about how NO may affect remodelling in the distal lung. We hypothesized that there is a link between alveolar NO and remodelling processes in the distal lung of mild asthmatics.

Method: Transbronchial biopsies were obtained from patients with mild asthma $(n=7)$ and healthy subjects $(n=8)$. Exhaled NO was measured at different flow rates and alveolar NO concentrations were calculated using a linear model. For studies of remodelling processes in the distal lung, primary fibroblasts were grown from biopsies and labelled with radioactive sulphur (35S) to measure changes in the synthesis of extracellular matrix proteins such as proteoglycans. To study the effect of NO on proteoglycan production, fibroblasts were cultured with unselective and selective nitric oxide synthase (NOS) inhibitors or a NO-donor. The expression of inducible NOS (iNOS) and synthesis of NO was measured.

Results: Production of the proteoglycans perlecan, biglycan and decorin in fibroblasts from patients with mild asthma correlated negatively to alveolar NO (perlecan: $P=0.01$, biglycan: $P=0.001$, decorin: $P=0.01$ ). Treatment with the specific iNOS inhibitor $1400 \mathrm{~W}$ significantly increased synthesis of the proteoglycans versican $(P=0.05)$, biglycan $(P=0.032)$ and decorin $(P=0.032)$ in fibroblasts from patients with mild asthma whereas fibroblasts from control subjects did not respond to either NOS inhibition or NO stimulation. There were also differences in NO production between fibroblasts from patients with mild asthma and healthy subjects. The fibroblasts expressed low amounts of iNOS.

Conclusion: Our data suggest that there is a link between alveolar NO and remodelling in the distal lung of mild asthmatics and that iNOS may be involved in the remodelling process by reducing the amount of synthesised matrix. 


\section{Oral Abstract Session}

\section{OAS 17 - Contact allergic dermatitis}

\begin{abstract}
95
In-house validation and technology transfer of the GARD assay for prediction of sensitising compounds

Forreryd, A; Johansson, H; Rydnert, F; Albrekt, AS ; Borrebaeck, CAK; Lindstedt, $M$

Department of Immunotechnology, Lund University, Lund, Sweden
\end{abstract}

Background: Allergic contact dermatitis is caused by an adverse immune response towards chemical haptens. The disease affects a significant proportion of the population, with increasing incidences, which leads to a substantial economic burden for society. New legislations on the registration and use of chemicals within chemical and cosmetic industries require development of alternative, high-throughput, in vitro assays for the prediction of sensitisation, to replace current animal-based experiments.

Method: We have developed a human cellbased assay for the prediction of sensitising chemicals, called Genomic Allergen Rapid Detection, GARD. By analyzing the transcriptome of the MUTZ-3 cell line after $24 \mathrm{~h}$ stimulation, using well characterised skin sensitising chemicals $(N=20)$ and controls $(N=20)$, we have identified a genomic biomarker signature with potent discriminatory ability. In order to further adapt the assay to a high-throughput screening mode, we evaluated the performance of three nonarray based platforms using a restricted set of probes from the biomarker signature.

Results: The prediction accuracy of the assay has been assessed in three separate in-house, partially blinded, validation studies $(N=37)$, and is thus far $89 \%$. Results from the evaluation of platforms mimicked previous data from genome wide transcriptome analysis in terms of reproducibility and robustness while alternative platforms proved to be superior in terms of cost efficiency, increased sample throughput and simplified protocols.

Conclusion: The GARD assay was demonstrated to have potent ability to predict sensitisation. By changing the technical platform for gene expression analysis, we could retain the robustness and discriminatory power of GARD and at the same time simplify assay procedures, reduce assay cost and increase sample throughput providing a first step towards formal validation and adaption of the assay for industrial screening of potential sensitisers.

96

Presence of formaldehyde in cosmetic products made in Lithuania

Malinauskiene, $L^{1,2}$; Blaziene, $A^{1}$; Chomiciene, $A^{1}$; Isaksson, $\mathrm{M}^{2}$

${ }^{1}$ Center of Pulmonology and Allergology, Vilnius University, Vilnius, Lithuania; ${ }^{2}$ Department of Occupational and Environmental Dermatology, Lund University, Skåne University Hospital, Malmö, Sweden

Concomitant contact allergy to formaldehyde and formaldehyde-releasers remains common among patients with allergic contact dermatitis. Concentration of free formaldehyde in cosmetic products within allowed limits have been shown to induce dermatitis from short-term use on normal skin.

The aim of this study was to investigate the formaldehyde content of cosmetic products made in Lithuania. 42 samples were analysed with the chromotropic acid (CA) method for semi-quantitative formaldehyde determination. These included 24 leave-on (e.g., cremes, lotions) and 18 rinse-off (e.g., shampoos, soaps) products. Formaldehyde releasers were declared on the labels of ten products. Free formaldehyde was found in 11 products. No formaldehyde releaser was declared on the label of the only face creme investigated, but levels of free formaldehyde with the CA method was $>40 \mathrm{mg} / \mathrm{ml}$ and when analyzed with a high-performance liquid chromatographic method $-532 \mathrm{ppm}$. According to the EU cosmetic directive, if the concentration of formaldehyde is above $0.05 \%$ a cosmetic product must be labelled 'contains formaldehyde'.

It could be difficult for patients allergic to formaldehyde to avoid contact with products containing it as its presence cannot be determined from the ingredient labelling with certainity. The CA method is a simple and reliable method for detecting formaldehyde- presence in cosmetic products.

97

Type-IV sensitisation profiles are different in the anal and genital area

Bauer, $A^{1}$; Spornraft-Ragaller, $P^{1}$; Herschel, $S^{1}$; Beissert, $\mathrm{S}^{1} ;$ Geier, $\mathrm{J}^{2}$

${ }^{1}$ Technical University Dresden, Dermatology, University Hospital Carl Gustav Carus, Dresden, Germany;

${ }^{2}$ Information Network of Departments of Dermatology (IVDK), University of Goettingen, Goettingen, Germany

Background: Genital and anal skin is exposed to many different allergens in hygiene products, topical medicaments, ointments and textiles.

Method: To identify the most frequent allergens, data of the Information Network of Departments of Dermatology (IVDK) were analysed. Patch test results in 1001 patients with suspected contact allergy in the genital or anal area (genital $n=550$; anal $n=451$ ) patch tested in 47 dermatological departments from 2009 to 2012 were included. All patients without involvement of the anal or genital area patch tested during this time period formed the control group ( $n=49818)$.

Results: In the study group with anal involvement, there were significantly more men $(n=270,59.9 \%)$ than in the group with genital complaints $(n=206,37.5 \%)$. Allergic contact dermatitis was significantly more frequently diagnosed in patients with anal complaints $(149 / 451=33.5 \%)$ than in the group with genital dermatoses (130/ $550=23.6 \%$ ). Patients suffering from anal dermatoses showed significantly higher age- and sex-standardised sensitisation rates to Methylchloroisothiazolinone/Methylisothiazolinone (MCI/MI 8.2\%; 95\% $\mathrm{CI}$, $4.5-11.9 \%$ ) and Methylisothiazolinon (MI $9.7 \% ; 95 \% \mathrm{CI}, 5.4-14.0 \%$ ) than patients with genital involvement (MCI 2.7\%; 95\% CI, $1.3-4.1 \%$; MI 3.4\%; 95\%CI, $1.6-$ $5.2 \%$ ) and controls (MCI 3.3\%; 95\% CI, $3.1-3.5 \%$; MI 3.7\%; 95\% CI, 3.5-3.9\%). Sensitisation to Bufexamac also differed significantly between patients with anal $(7.5 \%)$ and genital $(0.9 \%)$ dermatoses. 
Type IV-sensitisation to local anaesthetics in more than $1 \%$ of the respective group was only seen in patients with anal involvement. Thirteen patients were sensitised to cincochaine, which is $9.3 \%$ of those tested $(n=140)$. Of 142 patients tested with lidocaine, seven were sensitised $(4.9 \%)$, and five out of 169 patients $(3.0 \%)$ reacted to benzocaine.

Conclusion: Summed up, patients with dermatitis of the anal area are more frequently sensitised to ingredients of hygiene products and topically applied drugs than patients with genital dermatoses.

98

Molecular basis of nickel allergy

Yin, L; Crawford, F; Marrack, P; Kappler, J; Dai, S Immunology, National Jewish Health, Denver, CO, United States

Background: Metal-induced allergic contact dermatitis results in a wide range of cutaneous reactions following dermal and systemic exposure. $\mathrm{Ni}^{2+}$ allergy is the most common cause of the contact dermatitis, and afflicts around $10-15 \%$ of the human population. Apart from the significance of $\mathrm{Ni}^{2+}$ in developing contact dermatitis, $\mathrm{Ni}^{2+}$ hypersensitivity in patients undergoing joint replacement and tooth implants is a severe problem. $\mathrm{Ni}^{2+}$ allergy is mediated by $\mathrm{T}$ cells, but how the $\mathrm{T}$ cell receptor (TCR) recognises $\mathrm{Ni}^{2+}$ is unknown.

Method: We are studying a TCR, ANi2.3, from an allergic patient that recognises $\mathrm{Ni}^{2+}$ bound to the MHCII molecule, DR52c, containing an unknown self-peptide. We have identified $\mathrm{Ni}^{2+}$-independent peptide mimotopes for the ANi2.3 TCR by using baculovirus peptide/DR52c libraries. These peptides satisfy the requirements of the TCR both for peptide and $\mathrm{Ni}^{2+}$. We determined the crystal structures of the DR52c/ $\mathrm{Ni}^{2+}$ independent mimotopes, and ANi2.3 TCR bound to DR52c and one of these $\mathrm{Ni}^{2+}$-independent mimotope peptide.

Results: We identified mimotope peptides that can replace both the self-peptide and $\mathrm{Ni}^{2+}$ in this ligand. They share a p7 lysine, whose $\varepsilon \mathrm{NH} 2$ group is surface exposed when bound to DR52c. While the TCR uses germline CDR $1 / 2$ amino acids to dock in the conventional diagonal mode on the mimotope-DR52c complex, the interface is dominated by the TCR V $\beta C D R 3$ interaction wth the p7 lysine. Mutations in the TCR CDR loops have similar effects on the $\mathrm{T}$ cell response to either the mimotope or $\mathrm{Ni}^{2+}$ ligand. The pocket of $\mathrm{p} 7 \mathrm{~K}$ of the mimotope bound to DR52c lays in a wide space between the arch of the $\beta 1$ helix and the side chains of p4 and p7 of the peptide. We have previously shown that this is the functional site of $\mathrm{Be}^{2+}$ binding to DP2.

Conclusion: We suggest the mimotope p7 lysine mimics $\mathrm{Ni}^{2+}$ in the natural TCR ligand and that MHCII $\beta$ chain flexibility in the area around the peptide p7 position forms a common site for cation binding in metal allergies.

\section{9}

\section{Innate lymphoid cells (ILC) in allergic} contact dermatitis

van de Poel, $\mathrm{S}^{1,2} ;$ Kunz, $\mathrm{S}^{1}$; Martin, $\mathrm{SF}^{1} ;$ Jakob, $\mathrm{T}^{1}$ Department of Dermatology, Allergy Research Group, Medical Center - University of Freiburg, Freiburg, Germany; ${ }^{2}$ Faculty of Medicine and Biology, University of Freiburg, Freiburg, Germany

Innate lymphoid cells (ILC) are a recently identified family of heterogeneous innate immune cells belonging to the lymphoid lineage. In analogy to $\mathrm{T}$ helper cell subsets, ILC can be classified into three groups based on the developmental dependency on transcription factors and expression of cytokines. Prototypical ILC are NK cells (ILC1), natural helper cells (ILC2) and lymphoid tissue-inducer (ILC3) cells. ILC are known to play a role in lymphoid organogenesis, tissue remodeling and inflammation including allergic asthma and reside especially in the intestine and the lung. Recent studies showed that ILC are also present in skin and mediate pathology in a mouse model of atopic dermatitis as well as in psoriatic plaque formation.

Here we analyzed the involvement of ILC in allergic contact dermatitis using the mouse model of TNCB-induced contact hypersensitivity. Using flow cytometry, we identified all three groups of ILC in the skin of naive BALB/c mice. Quantification of these cells using counting beads revealed a frequency of approximately 1150 ILC2, 350 ILC3, and 700 NK cells per $50 \mathrm{mg}$ ear skin. During the elicitation phase of contact hypersensitivity we observed a distinct increase of NK cells in the inflamed skin, their number peaking at around $24 \mathrm{~h}$ after challenge. The frequency of ILC2 cells in the skin showed a slight increase $24 \mathrm{~h}$ after challenge, whereas no alterations in the frequency of ILC3 cells could be observed. Analysis of the activation profile using ICOS as an activation marker revealed a higher activation of ILC2 cells in mice with contact hypersensitivity compared to control groups. Upon primary contact with the allergen or an irritant, we observed no difference in the frequency of ILC2 cells, whereas ILC3 and NK cell increased at later time points.

In conclusion, our data indicate that numbers and activation profile of dermal ILC are influenced by contact allergens and thus, ILC might be functionally involved in allergic contact dermatitis.

\section{0 \\ Detection of contact allergy to palladium: sodium tetrachloropalladate is better than palladium chloride}

Spiewak, R ${ }^{1,2}$; Samochocki, $Z^{3}$; Pasnicki, $\mathrm{M}^{4}$; Czarnobilska, $E^{5}$; Grubska-Suchanek, $E^{6}$; Bukiel, $\mathrm{M}^{7}$. Jedrzejewska-Jurga, $\mathrm{K}^{8}$; Czarnecka-Operacz, $\mathrm{M}^{9}$; Cisowska, $\mathrm{A}^{10} ;$ Niklasson, $\mathrm{B}^{11}$

${ }^{1}$ Department of Experimental Dermatology and Cosmetology, Jagiellonian University, Krakow, Poland:

${ }^{2}$ Research Department, Institute of Dermatology, Krakow, Poland; ${ }^{3}$ Dermatology Clinic, Medical University of Warsaw, Warsaw, Poland; ${ }^{4}$ AlergicusDent, Zary, Poland; ${ }^{5}$ Department of Clinical and Environmental Allergology, Jagiellonian University Medical College, Krakow, Poland; ${ }^{6}$ Dermatology Clinic, Medical University of Gdansk, Gdansk, Poland; ${ }^{7} \mathrm{NZOZ}$ Alergologia-Pulmonologia, Stargard Szczecinski, Poland; ${ }^{8}$ Lower-Silesian Centre for Occupational Medicine, Wroclaw, Poland: ${ }^{9}$ Dermatology Clinic, Medical University of Poznan, Poznan, Poland; ${ }^{10}$ Dermatology Practice, Kamienna Gora, Poland; ${ }^{11}$ Chemotechnique Diagnostics, Vellinge, Sweden

Background: Contact allergy to palladium emerges as a relevant concern for public health due to the increasing environmental exposure (nickel-free jewellery gold alloys, electronic devices including mobile phones, computers and LED television sets, palladium release from car exhaust converters). Until now, patch test with palladium chloride was the common way of diagnosing palladium allergy, however, newer data suggest that sensitivity of this test may be too low. With this regard, sodium tetrachloropalladate seems a better option. This study was aimed at comparing results of patch tests to palladium chloride and sodium tetrachloropalladate in a large population of eczema patients.

Method: In phase 2 of the KRAK study, patients with chronic/recurrent eczema were patch tested to both palladium(II) chloride $2 \%$ pet. (Chemotechnique Diagnostics; cat.-No. P-001) and sodium tetrachloropalladate(II) hydrate $3 \%$ pet. (S017). The test substances were applied on the patient's back for 2 days in IQ Ultra chambers (Chemotechnique), with subsequent readings on days 3,5 and 8 . 
Results: 1026 patients were tested: 730 females and 296 males, aged 1-90 (median 40) years. Positive reaction to at least one of the compared substances was recorded in 223 patients, including 81 who reacted to both formulations, 123 who reacted to S-017 only, and 19 who reacted to P-001 only (chi2: $P<0.001$ ). The overall detec- tion rate was $19.9 \%$ for S-017 (204 positive reactions, including 50 rated as clinically relevant, and 48 as cross reactions), and $9.7 \%$ for P-001 (100 positive reactions, including 24 clinically relevant and 30 cross reactions). Testing to P-001 only would have missed 29 clinically relevant reactions detected with S-017 (2.8\% of all patients), while testing to S-017 only would have missed four relevant reactions to $\mathrm{P}-001$ $(0.4 \%)$.

Conclusion: Sodium tetrachloropalladate (II) hydrate $3 \%$ pet. is more effective than palladium chloride $2 \%$ pet. in the detection of contact allergy to palladium. 


\section{Oral Abstract Session}

\section{OAS 18 - Optimising the diagnosis of food allergic patients}

101

Double blind placebo controlled food challenge (DBPCFC) with soy in a multicentre setting: first data of the BASALIT trial

Treudler, $\mathrm{R}^{1}$; Franke, $\mathrm{A}^{2}$; Schmiedeknecht, $\mathrm{A}^{2}$; Holzhauser, $\mathrm{T}^{3}$; Vieths, $\mathrm{S}^{3}$; Worm, $\mathrm{M}^{4}$; Biedermann, $\mathrm{T}^{5}$; Werfel, $\mathrm{T}^{6}$; Jappe, $\mathrm{U}^{7}$; Ballmer-Weber, $\mathrm{B}^{8}$; Brehler, $\mathrm{R}^{9}$; Kleinheinz, $A^{10}$; Bauer, $A^{11}$; Schmitt, $J^{11}$; Brüning, $\mathrm{H}^{12}$; KleineTebbe, $\mathrm{J}^{13}$; Ring, $\mathrm{J}^{14}$; Rueff, $\mathrm{F}^{15}$; Saloga, $\mathrm{J}^{16}$; Schäkel, $\mathrm{K}^{17}$; Merk, $\mathrm{H}^{18}$; Simon, $\mathrm{JC}^{1}$; BASALIT Study Group ${ }^{1}$ Klinik für Dermatologie, Venerologie und Allergologie, Universität Leipzig, Leipzig, Germany; ${ }^{2}$ Zentrum für Klinische Studien, Universität Leipzig, Leipzig, Germany; ${ }^{3}$ Paul-Ehrlich-Institut, Langen, Germany; ${ }^{4}$ Charité - Universitätsmedizin, Berlin, Germany; ${ }^{5}$ Universitätshautklinik Tübingen, Tübingen, Germany; ${ }^{6}$ Medizinische Hochschule, Hannover, Germany; ${ }^{7}$ Forschungszentrum Borstel, Borstel, Germany; ${ }^{8}$ Universitätsspital, Zürich, Switzerland; ${ }^{9}$ Universität Münster, Münster, Germany; ${ }^{10} \mathrm{Klinikum}$ Buxtehude, Buxtehude, Germany; ${ }^{11}$ Technische Universität, Dresden, Germany; ${ }^{12}$ Tagesklinik für Allergie und Hautkrankheiten, Kiel, Germany; ${ }^{13}$ Allergie und AsthmaZentrum Westend, Berlin, Germany; ${ }^{14}$ LudwigMaximilians Universität, München, Germany;

${ }^{15}$ Technische Universität, München, Germany;

${ }^{16}$ Universitätsmedizin d. Johannes Gutenberg Universität, Mainz, Germany; ${ }^{17}$ Ruprecht-KarlsUniversität, Heidelberg, Germany; ${ }^{18}$ RWTH, Aachen, Germany

Background: The BASALIT trial ${ }^{1}$ (EudraCT 2009-011737-27) aims to determine efficacy of rBet v 1 SCIT on threshold levels (LOAEL) after soy food challenge in patients with birch-associated soy allergy. We present preliminary data on baseline DBPCFC.

Methods: 15 German Centres, 1 Swiss. Inclusion criteria: birch pollinosis, sIgE Bet v $1 \geq$ CAP 3 and Gly m $4 \geq$ CAP 2 . DBPCFC: 2 days, randomized sequence of placebo (PL) or soy meal (SOY) (produced centrally, powdered ingredients, water to be added). SOY included Supro ${ }^{\circledR}$ (Uelzena, Germany), $88 \%$ soy protein; Gly $\mathrm{m} 4$ levels were monitored. Nine dose levels at 20 min intervals $(0.00044 \mathrm{~g}-15 \mathrm{~g}$ soy protein; max. cumulative dosage $24.7 \mathrm{~g}$ ). Standardised documentation for obj./subj. symptoms $^{2}$, of which predefined levels led to randomization for intervention.

Results: Data base: DBPCFC-BL data of $138 / 196$ patients $(62 \% \mathrm{f}$, age $38 \mathrm{y}+/-13)$; 56 were finally recruited for SCIT. Maximum dose level reached in $75 \%$ after SOY, and $90 \%$ after PL; obj. symptoms in $46 \%$ resp. $20 \%$; median $[25 \% ; 75 \%$ ] dose levels $6[4 ; 7]$ and 4 [3;7] after SOY resp. PL; LO$\mathrm{AEL}_{\text {obj }} 24.7[2.2 ; 24.7] \mathrm{g}$ in all patients and
$4.7[0.7 ; 20.95] \mathrm{g}$ in the randomized. Subj. symptoms in 59\% (SOY) and 38\% (PL); median dose levels $5[4 ; 6]$ and $4[1.3 ; 7]$ after SOY resp. PL; LOAEL $_{\text {subj. }} 7.2$ [0.7;24.7]g in all patients but $1.45[0.33 ; 2.2]$ $\mathrm{g}$ in the randomized. In randomized cohort, $n=45(80 \%)$ showed objective and $n=51(91 \%)$ subjective symptoms at SOY. Conclusion: This is the first study to perform standardised DBPCFC with soy in a multicentre setting. In $20 \%$, PL led to objective and in $38 \%$ to subjective symptoms, underlining the need for double blinding. LOAELs within the randomized cohort were higher (LOAEL $_{\text {obj }} 4.7 \mathrm{~g}$, LO$\mathrm{AEL}_{\text {subj }} 1.45 \mathrm{~g}$ ) than previously shown ${ }^{3}$ with soy in chocolate bars, pointing out the importance of the food matrix. The effect of SCIT on threshold levels is under investigation.

Ref.: ${ }^{1}$ Treudler et al. Hautarzt 2012, ${ }^{2}$ Treudler et al. EAACI 2010, ${ }^{3}$ BallmerWeber et al. JACI 2007.

102

Analysis of vital signs in severe patients receiving double-blind placebo-controlled food challenge

Katsuhito, I; Tomoyuki, A; Shigehito, E; Kenichi, N; Yumi, K; Yu, O; Kiyotake, O; Sakura, S; Noriyuki, Y; Motohiro, E

Pediatrics, National Sagamihara Hospital, Kanagawa, Japan

Background: Oral food challenge (OFC) is the best way to identify causative foods for food allergy (FA) patients. It is necessary for physicians to perform OFC safely without inducing severe symptoms such as anaphylactic reactions (AN). There are few reports on usefulness of monitoring vital signs during OFC. We sought to determine whether monitoring vital signs such as blood pressure (BP), heart rate (HR), and body temperature (BT), would be useful to predict the severe allergic reactions during OFC.

Method: Our study consisted of 96 severe FA patients (median age, 7.8 years). Egg $(n=25)$, milk $(n=26)$, wheat $(n=21)$, and peanut $(n=24)$ provocations were performed by double-blind placebo controlled food challenge (DBPCFC) to identify threshold. We administered increasing doses of causative foods to subjects every
30 min. BP, HR, and BT were monitored every $5 \mathrm{~min}$ from the beginning to $120 \mathrm{~min}$. Challenges were stopped when objective symptoms had appeared. Percent changes from baseline and area under curve (AUC) of those values against time were calculated to evaluate the association of those change and severity of AN.

Results: Since the DBPCFC was for the purpose of oral immunotherapy, all subjects fulfilled the criteria of anaphylaxis. The mean $\%$ BP values were elevated significantly during active challenge compared with placebo $(\mathrm{Egg}, \quad 5.1 \pm 1.3$ vs $0.3 \pm 1.5$; milk, $5.6 \pm 1.3 \mathrm{vs}-3.0 \pm 1.5$; wheat, $3.6 \pm 1.7 \mathrm{vs}$ $-0.6 \pm 1.9$; peanut, $6.6 \pm 1.6 \quad$ vs $0.6 \pm 2.2 \%$ ). The AUC of $\% \mathrm{BP}$ was significantly elevated during egg, milk and peanut OFC compared with placebo (egg, $615.2 \pm 128.7 \quad$ vs $14.8 \pm 195.7 ; \quad$ milk, $503.1 \pm 127.7$ vs $-225.3 \pm 162.0$; peanut, $640.5 \pm 183.7$ vs $-43.4 \pm 139.1 \% 5 \mathrm{~min}$ ). The appearance of wheeze, vomiting, and systemic urticaria was related to BP increase. Early \%BP increase (15-30 min) preceding $\mathrm{AN}$ was detected in all positive challenges. Conclusion: Monitoring of vital signs, especially BP, during OFC is useful to predict the onset of objective allergic reactions.

\section{3 \\ Association of casein and beta- lactoglobulin specific IgE and IgG4 and serum basal tryptase levels with the reactivity to unheated and heated milk products (yoghurt, feta cheese and muffin) in children with IgE-mediated milk allergy}

Arik Yilmaz, E; Cavkaytar, O; Buyuktiryaki, B; Sekerel, BE; Soyer, O; Sackesen, C

Department of Pediatric Allergy, Hacettepe University School of Medicine, Ankara, Turkey

Background: It was recently shown that $80 \%$ of children with milk allergy could tolerate heated milk products. Yoghurt and cheese being one of the first main solid foods in infancy are unheated products of milk. We aimed to determine the children, who can tolerate unheated milk products by milk, casein, b-lactoglobulin sIgE and sIgG4 and serum basal tryptase (sBT) levels. 
Method: Food challenge tests were performed with muffin (heated milk), yoghurt and cheese (unheated milk), each containing $1 \mathrm{~g}$ milk protein. The children, who tolerated muffin, yoghurt and cheese were undergone DBPCFCT with pasteurised milk. The milk, casein, b-lactoglobulin sIgE, sIgG4 and sBT levels were determined by UniCAP method.

Results: Four groups of tolerance to milk was described: $a$.Tolerant to pasteurised milk $(n=58), \quad b$.Tolerant to muffinyoghurt-cheese, but reactive to pasteurised milk $(n=23), c$.Tolerant to muffin, but reactive to yoghurt-cheese $(n=17), d$. Reactive to muffin $(n=14)$. Levels of milk sIgE were significantly different among groups, respectively $\quad(P<0.001) \quad[5.2 \pm 1.4$; $16.2 \pm 5.1 ; \quad 29.4 \pm 9.8 ; \quad 53 \pm 14.2 \mathrm{kU} / \mathrm{L}$ (mean \pm SEM)]. Levels of casein $\operatorname{sIgE}$ and b-lactoglobulin sIgE were significantly different among groups, respectively [casein: $2.6 \pm 0.9 ; \quad 8.1 \pm 4.1 ; \quad 12.5 \pm 4.2$; $24.7 \pm 8.9 \mathrm{kUA} / \mathrm{L}, P=0.014$; b-lactoglobulin: $0.9 \pm 0.2 ; \quad 1.8 \pm 0.4 ; \quad 4.1 \pm 1.8$; $6.7 \pm 2.6, \quad P=0.013]$. The highest milk, casein and beta-lactoglobulin sIgE levels were obtained in children, who were reactive to muffin followed by children, who were reactive to yoghurt-cheese. Casein and b-lactoglobulin sIgG4 levels did not show significant difference among groups. The sBT levels showed a trend of increase among groups who are reactive to milk $(2.8 \pm 0.3 ; 3.5 \pm 0.6 ; 4 \pm 0.4 \mu \mathrm{g} / 1$, group b,c and d respectively, $P$ for trend=0.036).

Conclusion: Our results show that casein, b-lactoglobulin sIgE and tryptase levels may discriminate not only the children, who are tolerant to heated milk, but also tolerant to unheated milk products like yoghurt and feta cheese.

\section{4 \\ Clinical relevance of sensitisation to walnut components Jug r 1, 2 and/or 3}

Pascal, $\mathrm{M}^{1,2}$; Lozano, $\mathrm{J}^{3}$; Bartra, $\mathrm{J}^{2,4}$; Villarroel, $\mathrm{P}^{1}$; Dias, $\mathrm{M}^{3}$; Jiménez-Feijoo, $\mathrm{R}^{3}$; Domínguez, $\mathrm{O}^{3}$; Bosque, $\mathrm{M}^{5}$; Botey, $\mathrm{E}^{6}$; Cisteró, $\mathrm{A}^{6}$; Garcia-Moral, $\mathrm{A}^{2,4}$; SánchezLópez, $\mathrm{J}^{2,4}$; Vilella, $\mathrm{R}^{1,2}$; Juan, $\mathrm{M}^{1,2}$; Plaza, $\mathrm{AM}^{3}$; Yagüe, $\mathrm{J}^{1,2}$

${ }^{1}$ Hospital Clinic, Servei d'Immunologia, CDB, Barcelona, Spain; ${ }^{2}$ IDIBAPS, Universitat de Barcelona, Barcelona, Spain; ${ }^{3}$ Hospital Sant Joan de Déu, Servei d'Immunoal-lèrgia, Esplugues de Llobregat, Spain; ${ }^{4}$ Hospital Clínic, Unitat Al-lèrgia, Servei de Pneumologia, ICT, Barcelona, Spain; ${ }^{5}$ Hospital Universitari Parc Taulí, Pneumologia, Al-lèrgia i Immunologia Clínica Pediàtrica, Sabadell, Spain; ${ }^{6} \mathrm{Hospital}$ Universitari Quiron Dexeus, UAB, Al-lèrgia Dexeus, Barcelona, Spain

Background: Walnut allergy is commonly associated with severe, sometimes fatal, reactions and is one of the most frequent plant food allergies. Most walnut-allergic patients are sensitised to English walnut
(Juglans regia), the commercially available and widely consumed. Walnut allergenic components described are storage proteins (Jug r 1, 2 and 4), LTP (Jug r 3) and vicilins, although data on clinical relevance of sensitisation to them is limited.

Objective: To evaluate the clinical relevance of sensitisation to Jug r 1, 2 and 3 in children and adults.

Methods: Observational and descriptive study retrospectively analyzing the clinical relevance of Jug r 1, 2 and/or 3 sensitisation detected by multiplexed CRD (ImmunoCAPISAC, ThermoFisher Scientific) in children and adults.

Results: 99 children (11[4-18] years-old) and 72 adults (35[19-67] years-old) were analysed. Sensitisation profile varied among cohorts (children vs adults, \%): Jug r 1 (20 vs 0), Jug r 2 (10 vs 7), Jug r 3 (36 vs 85 ), Jug r $1 \& 2$ (6 vs 0 ), Jug r $1 \& 3$ (9 vs 4), Jug r $2 \& 3$ (6 vs 4), Jug r $1 \& 2 \& 3$ (12 vs $0)$. In children, Jug $\mathrm{r} 1$ was more frequent in allergic than tolerant subjects $(P=0.023)$ and related to systemic symptoms, whereas Jug r 3 sensitisation was more frequent in tolerant ones $(P<0.0004)$. Sensitisation to Jug r $1 \& 2 \& 3$ only occurred in allergic children (frequently anaphylaxis). In adults Jug r 1 sensitisation was not observed. Jug $\begin{array}{rll}\mathrm{r} & 3\end{array}$ sensitisation equally occurred among allergic and tolerant subjects and was related to mild and severe reactions. Jug r 2 sensitisation was clinically expressed as oral allergy syndrome.

Conclusion: Jug $\mathrm{r} 1$ was significantly more frequent and associated with severe reactions in children, whereas in adults barely existed. Sensitisation to Jug $r \quad 1 \& 2 \& 3$ increased the likely for walnut allergy in children. Jug r 3 was the most frequent in adults without differences between allergic and tolerant subjects, but in children it showed a higher association with tolerant ones. Prospective studies are required to address these observations.

\section{5 \\ Development of a quantitative multiplex immunoassay for food allergen proteins}

Kuklinska-Pijanka, A; Young, E; Chapman, MD; Hindley, JP

INDOOR Biotechnologies Ltd., Cardiff, United Kingdom

Background: In order to help allergic patients manage often severe symptoms, food manufacturers are required to list allergens on their products and researchers are working to develop effective immunotherapies. Due to limitations of the existing tools precise identification of the allergens in foods, therapeutic and diagnostic products can be difficult. We sought to develop an accurate, sensitive and reliable assay that would enable simultaneous quantification of multiple food allergens.

Method: Using microsphere technology we developed a 'proof of concept' multiplex immunoassay that enables simultaneous quantification of major peanut allergens: Ara h 1, Ara h 2 and Ara h 6. Fluorescent beads were coupled to allergen specific monoclonal antibodies. Detection of the target allergens was accomplished using biotinylated specific mono- or polyclonal antibodies and streptavadin conjugated fluorochrome. Highly purified natural allergens were used to generate a standard curve for each protein. Allergen content was measured in various sample types including NIST Standard Reference Material peanut butter, spiked samples and 'real foods'. The results were compared to ELISA.

Results: The multiplex assay was able to measure multiple allergens in a small, single sample making the technique highthroughput and costs effective compared to other methods. Preliminary tests show high correlation between the multiplex assay and ELISA $\left(R^{2}=0.82-0.98\right)$. Moreover, compared to ELISA, the sensitivity of the multiplex assay was increased by up to $40-$ fold.

Conclusion: The flexibility of the microsphere technology allows for expansion of the assay and will enable addition of further allergens (such as tree nuts, egg, milk and shrimp) to the list of analytes. Ultimately this quantitative multiplex tool for research, pharmaceutical, biotech and food industries will benefit allergy sufferers through improving therapeutics and diagnostics and by reducing the risk of inadvertent contamination of food.

\section{6 \\ Is the basophil activation test (BAT) a reliable tool in the diagnosis of soy allergy?}

Teodorowicz, $\mathrm{M}^{1}$; Breedveld, $\mathrm{AC}^{1}$; Jansen, $\mathrm{APH}^{2}$; Ruinemans-Koerts, $\mathrm{J}^{3}$; Wichers, $\mathrm{HJ}^{4}$; Savelkoul, $\mathrm{HFJ}^{1}$ ${ }^{1}$ Cell Biology and Immunology Group, Wageningen University and Research Centre, Wageningen, The Netherlands; ${ }^{2}$ Department of Otorhinolaryngology, Radboud University Nijmegen Medical Centre, Nijmegen, The Netherlands; ${ }^{3}$ Department of Clinical Chemistry and Haematology, Rijnstate Hospital, Arnhem, The Netherlands; ${ }^{4}$ Food and Biobased Research, Wageningen University and Research Centre, Wageningen, The Netherlands

Background: The basophil activation test (BAT) was shown to be a clinically useful diagnostic test for food allergy. Soy allergy is of particular importance because soy represents an insidious source of hidden allergens in many processed food. Thermal processing can cause conformational 
changes of proteins thereby changing its allergenic potential. The aim of the study was to use BAT to determine the influence of processing on allergenicity of soy proteins. The correlation between clinical profile of an individual patient and the BAT results was analysed.

Method: Serum IgE levels against total soybean extract, Gly m 4, Gly m 5 and Gly $\mathrm{m} 6$ were measured in 27 patients with a clinical history of soy allergy. Two groups of patients were distinguished: only Gly $\mathrm{m} 4$ positive $(81.5 \%)$ and Gly $\mathrm{m} 5 / 6$ positive $(18.5 \%)$. Five patients from each group were selected for BAT analysis that included the purified native allergens and five differently processed soy protein extracts (SPE).

Results: Patients who were only Gly $\mathrm{m} 4$ positive $(1.43-8.3 \mathrm{kU} / 1)$ were negative in BAT. Gly $\mathrm{m} 5 / 6$ positive patients were also positive in BAT. The optimal concentrations of raw SPE was evaluated individually for each patient and varied from 1$500 \mathrm{ng} / \mathrm{ml}$. Percentage of basophil stimulation by raw SPE was patient and treatment-dependent and reached app. $60 \%$ in case of highly allergic individuals. The reduction of allergenicity up to $100 \%$ was observed when SPE was heated. However, we also showed the presence of neo-allergens formed during processing which were able to activate basophils stronger than raw SPE.

Conclusion: To obtain objective BAT results for Gly $\mathrm{m} 5$ and 6 positive patients, the concentration curve of SPE should be performed for each patient individually. Processing of SPE significantly influenced the percentage of basophils stimulation, which emphasizes the importance of the standardisation of extracts quality between different laboratories.

*Suported by EU-FP7-PEOPLE-2011IEF (302295) 


\section{Oral Abstract Session}

\section{OAS 19 - Allergic immune responses}

\begin{abstract}
107
Allergic disease-related phenotypic differences emerged in the TH2 subset: steps toward a $T$ cell biomarker for allergy

Wambre, E1; Bajzik, $\mathrm{V}^{1}$; Robinson, $\mathrm{D}^{2}$; Kwok, $\mathrm{W}^{1}$ 'Benaroya Research Institute, Seattle, WA, United States; ${ }^{2}$ Virginia Mason Medical Center, Seattle, WA United States
\end{abstract}

Background: Identification of immunological signatures for allergic disease could enable objective assessment of disease status. In this study we sought to identify candidate $\mathrm{T}$ cell biomarkers that reflect the allergen-specific responses of allergic individuals and predict the clinical efficacy of allergen-specific immunotherapy.

Method: Ex vivo MHC-class II tetramer staining was used to detect, characterise and sort allergen-specific CD4+ $\mathrm{T}$ cells. Transcriptome and surface marker immuno-phenotyping of allergen-specific CD4+ T cells from allergic and non-allergic subjects revealed a 'pathogenic footprint' that could be analyzed by flow cytometry. These $\mathrm{T}$ cell biomarkers were then assessed in allergic individuals and non-allergic individuals to test for their ability to discriminate allergen-specific $\mathrm{T}$ cells from the rest of the $\mathrm{T}$ cell repertoire.

Results: We have identified a phenotypically distinct effector $\mathrm{TH} 2$ subset in allergic individuals that is virtually absent in nonatopic subjects. This allergic $\mathrm{T}$ cell signature is characterised by the unique and stable expression of five $\mathrm{T}$ cell surface markers and includes the vast majority of allergen-specific $\mathrm{TH} 2$ cells. As such, we denote cells with this surface marker expression the TH2A subset. Interestingly, similar allergic disease-related phenotypic differences also emerged in the type 2 innate lymphoid cell subset and our preliminary data supports clinical application of the TH2A cells as useful biomarker.

Conclusion: Modification of this 'allergic T cell signature' will help us to address a large unmet need in the prediction of the onset of allergy and clinical efficacy of allergy vaccine. Further study of this $\mathrm{T}$ cell subset could be of critical importance for understanding mechanisms of action associated with successful immunotherapy. Future vaccine strategies directed against the TH2A subset may be of value in allergic disorders.

108

Increased fecal calprotectin at age 2 months associates with early colonisation pattern and predicts atopic dermatitis and asthma by the age of 6 years

Orivuori, $\mathrm{L}^{1}$; Mustonen, $\mathrm{K}^{2}$; de Goffau, $\mathrm{M}^{3}$; Hakala, $\mathrm{S}^{1}$. Paasela, $\mathrm{M}^{1}$; Braun-Fahrländer, $\mathrm{C}^{4}$; Dalphin, J-C $\mathrm{C}^{5}$. Genuneit, $\mathrm{J}^{6}$; Lauener, $\mathrm{R}^{7,8}$; Riedler, $\mathrm{J}^{9}$; Weber, $\mathrm{J}^{10}$; von Mutius, $\mathrm{E}^{10}$; Pekkanen, $\mathrm{J}^{2}$; Harmsen, $\mathrm{H}^{3}$; Vaarala, $\mathrm{O}^{11}$; PASTURE Study Group

1Department of Vaccination and Immune Protection, National Institute for Health and Welfare, Helsinki, Finland; ${ }^{2}$ Environmental Health Department, Nationa Institute for Health and Welfare, Kuopio, Finland; ${ }^{3}$ Department of Medical Microbiology, University Medical Center Groningen and University of Groningen, Groningen, The Netherlands; ${ }^{4}$ Swiss Tropical and Public Health Institute, Basel, Switzerland ${ }^{5}$ The Department of Respiratory Disease, Université de Franche-Comté, University Hospital, Besançon, France; ${ }^{6}$ Institute of Epidemiology and Medical Biometry, UIm University, Ulm, Germany; ${ }^{7}$ Children's Hospital of Eastern Switzerland, St. Gallen, Switzerland; ${ }^{8}$ Christine Kühne-Center for Allergy Research and Education, Hochgebirgklinik Davos, Davos, Switzerland; ${ }^{9}$ Children and Young Adults' Medicine, Children's Hospital, Schwarzach, Austria; ${ }^{10}$ Dr. von Hauner Children's Hospital of the Ludwig Maximilian University Munich, Munich, Germany; ${ }^{11}$ National Institute for Health and Welfare, Department of Vaccination and Immune protection, Helsinki, Finland

Background: Calprotectin is an antimicrobial protein secreted by neutrophils. Fecal calprotectin is a biomarker of intestinal inflammation and useful in the diagnosis and follow-up of inflammatory bowel disease.

Method: We studied 762 families participating in the EFRAIM study in Austria, Finland, Germany, and Switzerland. Fecal samples were collected from infants at the age of 2 months and analyzed for calprotectin using the ELISA method. To address the effect of gut microbiota on fecal calprotectin, the composition of fecal microbiota was analyzed in a subgroup of 120 infants with $16 \mathrm{~S}$ RNA pyrosequencing. Atopy in children was defined by specific IgE levels in serum collected at the age of 6 years. Data of environmental factors, doctor-diagnosed atopic dermatitis (AD) and asthma were collected by questionnaire. Multivariate logistic regression models were used for analysis. The effect of
E. coli LPS on in vitro monocyte IL-10 secretion was studied by flow cytometry. Results: Infants from farming environments had higher levels of fecal calprotectin when compared to the infants from non-farming environments $(P=0.003)$. Elevated fecal calprotectin was associated to environmental conditions indicating increased bacterial load. Infants with calprotectin above the 90th percentile, had an increased risk of developing $\mathrm{AD}$ and asthma/asthmatic bronchitis by the age of 6 years [aOR $1.97(1.04-3.77)$ and 2.49 (1.22-5.10), respectively]. High fecal calprotectin correlated positively with the abundance of Lactobacillus sp., and negatively with the abundance of Escherichial Shigella groups. LPS from E. coli stimulated production of IL-10 in monocytes. Conclusion: Remarkable intestinal inflammation, i.e. fecal calprotectin levels above the 90th percentile at 2 months of age, predicted asthma and $\mathrm{AD}$ by the age of 6 years, and was linked to early colonisation with Lactobacillus sp. and low abundance of fecal Escherichia/Shigella. LPS from $E$. coli induced IL-10 activation in monocytes which could explain the downregulation of intestinal inflammation associated with Escherichia colonisation.

\section{9 \\ Treatment effects of a GATA-3-specific DNAzyme on disease progression in a subacute oxazolone-induced mouse model of atopic dermatitis}

Purath, $U^{1}$; Ibrahim, $\mathrm{R}^{2}$; Turowska, $A^{1}$; Homburg, $U^{1}$; Runkel, $\mathrm{F}^{3}$; Schmidts, $\mathrm{T}^{3}$; Dobler, $\mathrm{D}^{3}$; Renz, $\mathrm{H}^{2}$; Garn, $\mathrm{H}^{2}$; Mueller, $A^{1}$

Sterna Biologicals GmbH \& Co. KG, Marburg, Germany: ${ }^{2}$ Institute of Laboratory Medicine and Pathobiochemistry, Philipps University of Marburg Medical Faculty, Marburg, Germany; ${ }^{3}$ University of Applied Sciences, Giessen, Germany

DNAzymes represent a particular class of antisense molecules combining the specificity of antisense molecules with an inherent catalytic activity which makes them an attractive tool for highly specific interference with disease-causing target molecules. In general, they are single-stranded DNA molecules with two sequence-specific RNAbinding domains flanking a central catalytic domain. We developed and patented a 
DNAzyme - named hgd40 - that targets GATA-3, the central transcription factor in $\mathrm{T}$ helper cell type 2 (Th2) differentiation and activation. For penetration enhancement and DNAzyme protection a specific formulation for topical dermal application was developed and patented.

Targeting GATA-3 might be a key for therapeutic interventions in predominantly Th2-driven diseases like atopic dermatitis. For this reason, an oxazolone-induced contact hypersensitivity mouse model was modified to establish elongated skin swelling reactions compared to the acute models, thereby enabling the analysis of treatment effects on $\mathrm{T}$ cell-mediated pathomechanisms. The therapeutic effects of hgd40 were analyzed in this model with regard to clinical symptoms and the expression pattern of relevant transcription factors and cytokines over time.

Prophylactic treatment with topically applied hgd40 in a water/oil/water emulsion, specifically developed for penetration enhancement and DNAzyme protection, significantly and dose dependently reduced oxazolone-induced reactions in the skin, e.g., skinfold thickness. Molecular analysis revealed reduced GATA-3 mRNA levels early after topical application of hgd40.

In summary, the water/oil/water-formulated DNAzyme hgd40 may represent a new and promising therapeutic agent for the topical treatment of allergic skin diseases.

\section{0 \\ Activin-A drives the generation of human $\mathrm{CD4}^{+}{ }^{+} \mathrm{COS}^{+} \mathrm{IL}-10$-secreting regulatory $\mathrm{T}$ cells that suppress asthma in an aryl hydrocarbon receptor dependent pathway \\ Tousa, $\mathrm{S}^{1}$; Semitekolou, $\mathrm{M}^{1}$; Morianos, $\mathrm{I}^{1}$; Trochoutsou, $\mathrm{Al}^{1}$; Banos, $\mathrm{A}^{2}$; Brodie, $\mathrm{T}^{3}$; Kapasa, $\mathrm{M}^{2}$; Poulos, $\mathrm{N}^{1}$; Samitas, $\mathrm{K}^{4}$; Paraskevopoulos, $\mathrm{G}^{5}$; Gaga, $\mathrm{M}^{4}$; Hawrylowicz, $\mathrm{CM}^{6}$; Sallusto, $\mathrm{F}^{3}$; Xanthou, $\mathrm{G}^{1}$ ${ }^{1}$ Biomedical Research Foundation of the Academy of Athens (BRFAA), Cellular Immunology Laboratory, Athens, Greece; ${ }^{2}$ Biomedical Research Foundation of the Academy of Athens (BRFAA), Athens, Greece; ${ }^{3}$ Institute for Research in Biomedicine (IRB), Bellinzona, Switzerland; ${ }^{4}$ Athens Chest Hospital 'Sotiria', Athens, Greece, ${ }^{5} 401$ General Military Hospital of Athens, Athens, Greece; ${ }^{6}$ King's College London, London, United Kingdom}

Background: Regulatory $\mathrm{T}$ cells (Tregs) are crucial for asthma control. Our previous studies have shown that activin-A induces mouse Tregs that protect from asthma in vivo. Still, whether activin-A can induce human Tregs remains elusive.

Method: Peripheral blood $\mathrm{CD}^{+}{ }^{+} \mathrm{T}$ cells of atopics and asthmatics were stimulated with allergen. Phenotype and suppressive function of activin-A-treated $\mathrm{T}$ cells on allergic responses in vitro were studied. Using a humanized mouse model of asthma, we examined the effects of activinA-treated $\mathrm{T}$ cells in asthma protection in vivo.

Results: Our data reveal that activin-A inhibits human $\mathrm{T}$ cell responses during stimulation with allergen in vitro. In fact, activin-A induces $\mathrm{CD} 4{ }^{+} \mathrm{ICOS}^{+} \mathrm{IL}$ $10^{+}$Tregs (act-A-iTregs) that are hyporesponsive, do not produce Th effector cytokines and suppress allergen-driven proliferation and Th2 cytokine release by $\mathrm{T}$ responders. Using a humanized mouse model of asthma, we show that adoptive transfer of act-A-iTregs in vivo in prevention and therapeutic protocols ameliorates cardinal asthma features, such as airway hyperresponsiveness and lung inflammation. Of clinical relevance, act-A-iTregs restrain Th2 responses of severe asthmatics ex vivo. Mechanistic experiments reveal that act-A-iTregs do not express Th-associated transcription factors (TFs) but exhibit a major mRNA induction of the TF, aryl hydrocarbon receptor (AhR). Addition of an AhR antagonist reverses activin-A-mediated induction of $\mathrm{ICOS}^{+} \mathrm{IL}-10^{+}$Tregs, indicating a novel link between activin-A and AhR in human Treg generation. Notably, ChIP assays show that activin-A regulates IL-10 and ICOS expression in human Tregs through induction of AhR and interferon regulatory factor 4 binding to the respective gene promoters.

Conclusion: Our data introduce activin-A as a novel inducer of human $\mathrm{ICOS}^{+} \mathrm{IL}$ $10^{+}$Tregs that restrain allergic asthma. These findings may facilitate the use of actA-iTregs in adoptive-transfer therapıes aiming to re-establish tolerance in asthma.

\section{1 \\ Interleukin 10 signaling in $\mathrm{T}$ cells, B cells or neutrophils/monocytes is not essential to induce allergen specific tolerance}

Preuhsler, $\mathrm{A}^{1,2} ; \mathrm{Kunz}, \mathrm{S}^{1}$; Müller, $\mathrm{W}^{3}$; Jack, $\mathrm{RS}^{4}$; Martin, $\overline{S F}^{1}$; Roers, $A^{5}$; Jakob, $T^{1}$

${ }^{1}$ Department of Dermatology, Medical Center University Freiburg, Freiburg, Germany; ${ }^{2}$ University Freiburg, Biology, Freiburg, Germany; ${ }^{3}$ Faculty of Life Sciences, University of Manchester, Manchester, United Kingdom; ${ }^{4}$ Department of Immunology, Institute of Immunology and Transfusion Medicine, University Hospital Greifswald, Greifswald, Germany; ${ }^{5}$ Medical Faculty Carl Gustav Carus, Institute of Immunology, University of Technology Dresden, Dresden, Germany

Human studies suggest that allergen immunotherapy leads to regulatory immune responses that suppress the development of allergic inflammation. Interleukin (IL)-10 production by allergen-specific $\mathrm{T}$ cells has been suggested as one of the regulatory mechanisms. This was supported by in vivo studies in mice in which treatment with IL10 receptor (IL-10R) blocking antibody abrogated the beneficial effects of immunotherapy. In the present study we address the cellular targets of IL-10 in the process of tolerance induction by using mice with a cell type specific inactivation of the IL-10R gene generated by Cre/loxP-mediated recombination.

Ovalbumin (ova) sensitised mice were treated with three s.c. ova injections on alternate days for tolerance induction. After challenge by ova inhalation allergen specific antibody and cytokine responses as well as allergen induced airway inflammation were analyzed. Tolerance induction was effective in the suppression of airway inflammation in wildtype mice but not in IL-10R null mutants (IL-10R ${ }^{\mathrm{FL} / \mathrm{FL}}$ Cre deleter ${ }^{+}$), confirming the involvement of IL$10 \mathrm{R}$ in tolerance induction. In contrast, in mice that lack IL-10 signaling specifically in $\mathrm{T}$ cells $\left(\mathrm{IL}-10 \mathrm{R}^{\mathrm{FL} / \mathrm{FL}} \mathrm{CD} 4-\mathrm{Cre}^{+}\right.$) the degree of tolerance induction was comparable to that of Cre negative littermate controls both displaying strongly reduced eosinophilic infiltration into the bronchoalveolar space and reduced Th2 responses to allergen specific restimulation. Effective tolerance induction was also observed in mice with a $B$ cell specific (IL-10R $\mathrm{R}^{\mathrm{FL} / \mathrm{FL}}$ CD19-Cre ${ }^{+}$) as well as in mice with a neutrophil/monocyte specific (IL-10R $\mathrm{R}^{\mathrm{FL} / \mathrm{FL}}$ LysM-Cre ${ }^{+}$) deletion of the IL-10R.

In summary, our results show that in the murine model of allergen induced airway inflammation direct effects of IL-10 on T cells, B cells or neutrophils/monocytes are not critical for tolerance induction. Thus different cellular targets of IL-10 are likely to be involved in the benefical effects of allergen specific tolerance induction.

\section{2 \\ Aspergillus fumigatus increases BAFF expression on basophils from allergic and non allergic subjects}

Boita, $\mathrm{M}^{1}$; Pizzimenti, $\mathrm{S}^{1}$; Heffler, $\mathrm{E}^{1}$; Raie, $\mathrm{A}^{1}$; Saraci,

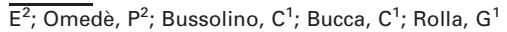
${ }^{1}$ Department Medical Science, University of Turin, Turin, Italy; ${ }^{2}$ Division of Hematology, University of Turin, Turin, Italy

Background: B-cell activating factor (BAFF) is known to play an important role in autoimmune diseases, while its role in allergic diseases is less known. BAFF is produced by cells of myeloid origin, like neutrophils, and recently membrane expression of BAFF has been observed in basophils of allergic and non allergic subjects. The stimuli which regulate the expression of BAFF on basophils membrane are not known, but neither IgE nor 
allergens (grass and ambrosia) have been found to increase membrane BAFF expression. Some allergens, like Aspergillus, are recognised by toll-like receptors (TLR-4 and -2 ) and polyclonal $\mathrm{IgE}$ production may be observed in asthmatic patients sensitised to Aspergillus, suggesting a possible role of BAFF in asthma with fungal sensitisation.

Aim: Whether IgE dependent and independent stimuli may up-regulate membrane expression of BAFF in basophils of patients with asthma and fungal sensitisation compared to healthy controls.
Patients and methods: Peripheral blood of 6 patients (age $60 \pm 16.88,3$ female) with asthma and Aspergillus sesitization and 6 healthy controls (age $40 \pm 15,4$ female) subjects was assessed for BAFF (CD257) expression by basophil activation test (BAT), before and after stimulation with IgE, Aspergillus and fMLP, SEB, LPS, 1-3 beta glucan, LTA-SA ((Lipoteicoic Acid from Staphilococcus Aureus) (IgE independent stimuli).

Results: BAFF expression was up-regulated by Aspergillus stimulation, but not by $\operatorname{IgE}$, both in asthmatics and controls. IgE independent stimuli, fMLP and SEB, were able to up-regulate BAFF expression in both groups. TLR-4 engagement by LPS did not increase membrane expression of BAFF, which was up-regulated by TLR-2 stimulation by $1-3$ beta glucan and by LTA-SA both in asthmatics and controls.

Conclusions: Basophils membrane expression of BAFF may be up-regulated by different non $\operatorname{IgE}$ stimuli in allergic and non allergic subjects. The increased expression of BAFF induced by Aspergillus is probably related to TLR-2 stimulation.

Supported by Fondazione Scientifica Mauriziana e Associazione Italiana Mastocitosi. 


\section{Oral Abstract Session}

\section{OAS 20 - Hereditary angioedema}

113

A nationwide survey of hereditary angioedema due to $\mathrm{C} 1$ inhibitor deficiency in Italy

Zanichelli, A; Arcoleo, F; Barca, M; Borrelli, P; Bova, M Cancian, M; Cicardi, M; Cillari, E; De Carolis, C; De Pasquale, T; Del Corso, I; Di Rocco, P; Guarino, M; Massaro, I; Minale, M; Montinaro, V; Neri, S; Perricone, R; Pucci, S; Quattrocchi, P; Rossi, O; Triggiani, M; Zanierato, G; Zoli, A

Dipartimento di Scienze Biomediche e Cliniche Luigi Sacco, Università degli Studi di Milano-Ospedale Luigi Sacco, Mlano, Italy

Background: Hereditary angioedema due to C1-inhibitor deficiency (C1-INH-HAE type I) or dysfunction (C1-INH-HAE type II) is a rare disease characterised by recurrent episodes of edema with an estimated frequency of 1:50 000 in the global population without racial or gender differences. In this study we present the results of a nationwide survey of HAE Italian patients followed up in 17 centers.

Method: All patients diagnosed with $\mathrm{C} 1$ INH-HAE from 1972 to 2013 were included in the study. Diagnosis of $\mathrm{C} 1$ INH-HAE was based on family and/or personal history of recurrent angioedema without urticaria and on antigenic and/or functional C1-INH deficiency.

Results: 963 patients were included in this survey: $47 \%$ male and $53 \%$ female. $94 \%$ were alive, median age 45 . Median age at diagnosis was 26. C1-INH-HAE type 1 were $87 \%$, median age at diagnosis 25 ; $\mathrm{C} 1$ INH-HAE type 2 were $13 \%$, median age at diagnosis 31 . Patients beloged to 376 unrelated families. Based on the Italian population our patients provide a minimum prevalence of 1:66 000. In late decades of life ( $>5$ th for men and $>6$ th for women) patients were less represented than general population. Functional C1INH was $\leq 50 \%$ in $99 \%$ of patients. Antigen C1INH was $\leq 50 \%$ in $100 \%$ of type 1 . C4 was $\leq 50 \%$ in $96 \%$ of patients.

Conclusion: Our data provide a minimum national prevalence of C1-INH-HAE similar to the 1:50 000 estimated prevalence. Life expectancy is shorter in C1-INH-HAE patients than in the general population. Less than $5 \%$ of C1-INH-HAE patients have $\mathrm{C} 4$ plasma levels $\geq 50 \%$ of normal.
114

Dose-ranging trial of a volume-reduced, subcutaneous formulation of a C1-INH concentrate (CSL830) for the prevention of acute attacks of hereditary angioedema

Zuraw, $\mathrm{B}^{1}$; Craig, $\mathrm{T}^{2}$; Longhurst, $\mathrm{H}^{3}$; Martinez-Saguer, $\mathrm{I}^{4}$; $\mathrm{Li}, \mathrm{H}^{5}$; Bernstein, $\mathrm{J}^{6}$; Staubach-Renz, $\mathrm{P}^{7}$; Levy, $\mathrm{R}^{8}$; Maurer, $\mathrm{M}^{9}$; Rehman, $\mathrm{S}^{10}$; Edelman, $\mathrm{J}^{11}$; Parasrampuria, $\mathrm{R}^{11}$; Feuersenger, $\mathrm{H}^{12}$; Cicardi, $\mathrm{M}^{13}$; for the COMPACT Investigators

${ }^{1}$ Department of Medicine, University of California San Diego, La Jolla, CA, United States; ${ }^{2}$ Penn State Hershey Medical Center, Hershey, PA, United States; ${ }^{3}$ London NHS Trust, London, United Kingdom; ${ }^{4} \mathrm{HZRM}$,

Mörfelden-Walldorf, Germany; ${ }^{5}$ Institute for Asthma \&Allergy, Chevy Chase, MD, United States; ${ }^{6}$ Bernstein Clinical Research Center, Cincinnati, OH, United States; ${ }^{7}$ Department of Dermatology, University of Mainz, Mainz, Germany; ${ }^{8}$ Allergy \& Asthma Center, Atlanta, GA, United States; ${ }^{9}$ Charité University, Berlin, Germany; ${ }^{10}$ Toledo Institute, Toledo, United States; ${ }^{11} \mathrm{CSL}$ Behring LLC, King of Prussia, PA, United States: ${ }^{12} \mathrm{CSL}$ Behring GmbH, Marburg, Germany; ${ }^{13}$ University Hospital Milano, Luigi Sacco, Milano, Italy

Background: Hereditary angioedema (HAE) manifests as recurrent swelling attacks that are often disabling and can be fatal. Long-term prophylaxis with twiceweekly intravenous injections of plasmaderived C1-Inhibitor (pdC1-INH) has been shown to be efficacious and safe for prevention of acute attacks. The safety and efficacy of a subcutaneously (sc) administered pdC1-INH has not been established. Method: This international, open-label, cross-over study (COMPACT Phase II) was conducted in 18 type I or II HAE patients who received twice-weekly doses of 1500,3000 or $6000 \mathrm{IU}$ of sc CSL830 for 4 weeks. The mean trough plasma levels of C1-INH functional activity (C1- F), C1INH- and $\mathrm{C} 4$-antigen levels $(\mathrm{C} 1$ - and $\mathrm{C} 4-$ A) during week 4 , and overall safety and tolerability were evaluated. The primary outcome was model-derived steady state trough values at week 4 .

Results: After sc CSL830 a dose-dependent increase of modeled [m] and observed trough functional C1-INH levels was seen. The 6000 IU dose achieved trough activity that was within the normal range for $\mathrm{Cl}$ INH functional activity. The s.c. administration of all three CSL 830 dosing regimens was safe and well tolerated. Inhibitory auto-antibodies to $\mathrm{C} 1-\mathrm{INH}$ were not observed.

Conclusions: The subcutaneous, volumereduced C1-INH concentrate (CSL 830) was well tolerated and led to a dose-dependent increase in physiologically relevant functional C1-INH plasma levels. Evaluation of the efficacy and safety of this new sc treatment modality warrants further investigation in a clinical outcome study of HAE patients suffering from frequent attacks.

\section{5}

Hereditary angioedema with normal C1 inhibitor in a French cohort: clinical characteristics and response to treatment with icatibant

Bouillet, L'; Boccon-Gibod, $I^{1}$; Launay, $D^{2}$; Gompel, $\mathrm{A}^{3}$; Kanny, $\mathrm{G}^{4}$; Fabien, $\mathrm{V}^{5}$; Fain, $\mathrm{O}^{6}$; for the IOS Study Group

${ }^{1}$ Grenoble University Hospital, Grenoble, France; ${ }^{2}$ Claude-Huriez Hospital, Lille, France; ${ }^{3}$ Paris Descartes University, Paris, France; ${ }^{4}$ Lorraine University, Nancy, France; ${ }^{5}$ Shire, Eysins, Switzerland; ${ }^{6}$ Jean-Verdier Hospital, Bondy, France

Background: Unlike hereditary angioedema (HAE) due to $\mathrm{C} 1$ inhibitor (C1-INH) deficiency (type I or II), HAE with normal C1-INH is characterised by normal C1INH function with recurrent, often severe/

\begin{tabular}{lllll}
\hline Dose & Reference Range & 1500 IU & 3000 IU & 6000 IU \\
\hline C1-F[m], \% (SD) & $70-130$ & $30.3(9.1)$ & $45.9(14.3)$ & $80.6(23.5)$ \\
C1-F, \% (SD) & $70-130$ & $31.7(10.0)$ & $44.3(17.7)$ & $80.5(25.0)$ \\
C1-A, g/l (SD) & $0.18-0.32$ & $0.06(0.02)$ & $0.15(0.13)$ & $0.23(0.16)$ \\
C4-A, mg/dl (SD) & $10-40$ & $11.1(4.5)$ & $14.1(5.3)$ & $18.4(6.6)$ \\
\hline
\end{tabular}

Pharmaco-kinetic and -dynamic results (mean; SD). 
very severe oedematous attacks. We analysed outcomes of icatibant-treated HAE type I/II or HAE with normal C1-INH attacks in a real-world setting.

Method: The Icatibant Outcome Survey [IOS; Shire, Eysins, Switzerland (NCT01034969)] is an international observational study monitoring safety and effectiveness of icatibant treatment. In France, icatibant is indicated for symptomatic treatment of acute HAE attacks in adults (with C1-INH deficiency). Retrospective analyses of IOS data (July 2009-September 2013) compared patients with HAE with normal C1-INH from a single centre (Grenoble) with all French patients with HAE type I/II. Descriptive analyses are reported as median (IQR).

Results: Patients with HAE with normal C1-INH were mostly women, and older at first symptoms and diagnosis vs patients with HAE type I/II (Table). Most icatibant-treated attacks affected the abdomen, skin and larynx and were severe/very severe (Table). For attacks in patients with HAE with normal C1-INH vs HAE type I/ II, median time to first icatibant injection was $1.0 \quad(0.5-3.5)$ vs $3.3 \quad(1.5-8.5) \quad \mathrm{h}$, $P=0.067$; median time to resolution was $26.6[8.3-46.0]$ vs $11.5[1.1-24.0] \mathrm{h}$, $P<0.001 \quad$ (severe/very severe attacks, $P<0.001)$; and median attack duration was $32.0(10.5-47.6)$ vs $18.3(10.1-31.5) \mathrm{h}$, $P=0.011$, respectively. A single icatibant injection was used for $76.8 \%$ of attacks in patients with HAE with normal C1-INH and $79.7 \%$ of HAE type I/II attacks.

Conclusion: Patients with HAE with normal C1-INH experienced longer attack duration and time to resolution compared with patients with HAE type I/II. Most attacks were successfully treated with a single icatibant injection.
116

Analysis of icatibant reinjection rates for the treatment of laryngeal hereditary angioedema attacks in the FAST-3 open-label extension study

Farkas, $\mathrm{H}^{1}$; Bernstein, $\mathrm{JA}^{2}$; Lumry, $\mathrm{WR}^{3}$; Baptista, $\mathrm{J}^{4}$; $\overline{\text { Riedl, } \mathrm{M}^{5}}$; Moldovan, $\mathrm{D}^{6}$

${ }^{1}$ Third Department of Internal Medicine, Semmelweis University, Budapest, Hungary; ${ }^{2}$ University of Cincinnati, Cincinnati, $\mathrm{OH}$, United States: ${ }^{3}$ AARA Research Center, Dallas, TX, United States; ${ }^{4}$ Shire, Lexington, MA, United States; ${ }^{5}$ University of California San Diego, CA, United States; ${ }^{6}$ Mures County Hospital, Tirgu-Mures, Romania

Background: Icatibant demonstrated efficacy for the treatment of attacks of hereditary angioedema (HAE) type I or II in the double-blind, placebo-controlled phase of the For Angioedema Subcutaneous Treatment-3 study (FAST-3; NCT00912093). In the controlled phase, only one icatibant injection could be administered for one attack in each patient; however, an openlabel extension (OLE) phase provided data for a larger number of attacks, with up to 3 injections per attack permitted. Here, the need for reinjection to treat potentially fatal laryngeal attacks in the FAST-3 OLE phase was analysed post hoc.

Method: Following the controlled phase of FAST-3, adults with HAE type I/II could receive up to 3 injections of open-label icatibant, administered by a healthcare professional at intervals of $\geq 6 \mathrm{~h}$, per attack in the OLE phase. Reinjection rates, time to onset of symptom relief $(\geq 50 \%$ reduction in patient-reported, composite visual analog scale [VAS] symptom score), and time to almost complete symptom relief (all VAS symptom scores $<10 \mathrm{~mm}$ ) were summarised for laryngeal attacks.

Results: In the OLE phase, 23 patients experienced laryngeal attacks (age $\leq 30$ years, $26.1 \%$, $>30-40$ years, $43.5 \%$, $>40-50$ years, $30.4 \%$; female, $56.5 \%$; HAE type I, $100 \%$ ). There were 43 laryngeal attacks (13 mild, 20 moderate, 10 severe), of which $41(95.3 \%)$ were treated with one icatibant injection. For attacks treated with one injection, median time to onset of symptom relief was $1.5 \mathrm{~h}$ [interquartile range (IQR), $1.0-2.3 ; n=40$ attacks] and median time to almost complete symptom relief was $3.5 \mathrm{~h}$ (IQR, 1.5-23.9; $n=39$ attacks). There were 2 mild-moderate laryngeal attacks $(2 / 43,4.7 \%)$ in 2 patients where a second injection was administered due to worsening of attack symptoms. No laryngeal attacks required 3 injections.

Conclusion: Icatibant provided symptom relief for mild-to-severe laryngeal HAE attacks in the FAST-3 OLE study. Almost all attacks were successfully treated with one injection.

\section{7}

The Icatibant Outcome Survey: treatment of laryngeal hereditary angioedema attacks

Longhurst, $\mathrm{H}^{1}$; Aberer, $\mathrm{W}^{2}$; Bouillet, $\mathrm{L}^{3}$; Caballero, $\mathrm{T}^{4}$; Maurer, $\mathrm{M}^{5}$; Fabien, $\mathrm{V}^{6}$; Zanichelli, $\mathrm{A}^{7}$; for the IOS Study Group

${ }^{1}$ Department of Immunology, Barts Health NHS Trust, London, United Kingdom; ${ }^{2}$ Medical University of Graz, Graz, Austria; ${ }^{3}$ Grenoble University Hospital, Grenoble, France; ${ }^{4}$ Hospital La Paz Institute for Health Research (IdiPAZ), Madrid, Spain; ${ }^{5}$ Charité - Universitätsmedizin Berlin, Berlin, Germany; ${ }^{6}$ Shire, Eysins, Switzerland;

${ }^{7}$ Università degli Studio di Milano, Milan, Italy

Background: Laryngeal attacks may be fatal without prompt and effective treatment. Icatibant is a bradykinin B2 receptor antagonist used to treat hereditary angioedema (HAE) type I/II attacks in adults. We analysed characteristics and outcomes of icatibant-treated laryngeal HAE attacks in a real-world setting.

Method: The Icatibant Outcome Survey [IOS; Shire, Eysins, Switzerland (NCT01034969)] is a prospective, international observational study to monitor the

\begin{tabular}{|c|c|c|c|c|c|c|c|c|c|}
\hline $\begin{array}{l}\text { HAE } \\
\text { Type }\end{array}$ & $\begin{array}{l}\text { Patients, } \\
N\end{array}$ & $\begin{array}{l}\text { Females, } n / N \\
(\%)\end{array}$ & $\begin{array}{l}\text { Median Age } \\
\text { at First } \\
\text { Symptoms } \\
\text { (IQR), years }\end{array}$ & $\begin{array}{l}\text { Median Age at } \\
\text { Diagnosis } \\
(\mathrm{IOR}) \text {, years }\end{array}$ & $\begin{array}{l}\text { Total } \\
\text { Icatibant- } \\
\text { Treated } \\
\text { Attacks, } \\
N\end{array}$ & $\begin{array}{l}\text { Abdominal } \\
\text { lcatibant- } \\
\text { Treated } \\
\text { Attacks, } n / N \\
(\%)\end{array}$ & $\begin{array}{l}\text { Cutaneous } \\
\text { lcatibant- } \\
\text { Treated } \\
\text { Attacks, } n / N \\
(\%)\end{array}$ & $\begin{array}{l}\text { Laryngeal } \\
\text { lcatibant- } \\
\text { Treated } \\
\text { Attacks, } n / N \\
(\%)\end{array}$ & $\begin{array}{l}\text { Severe/very } \\
\text { Severe } \\
\text { Icatibant- } \\
\text { Treated Attacks } \\
n / N(\%)\end{array}$ \\
\hline $\begin{array}{l}\text { HAE } \\
\text { with } \\
\text { normal } \\
\text { C1-INH }\end{array}$ & $\begin{array}{l}52 \text { (4 with } \\
\text { F12 } \\
\text { mutation) }\end{array}$ & $44 / 52(84.6)$ & $\begin{array}{l}21.5 \\
(16.0-35.0)\end{array}$ & $\begin{array}{l}32.4 \\
(23.5-41.1)\end{array}$ & $\begin{array}{l}138 \text { (23 } \\
\text { patients; } \\
\text { off-label } \\
\text { use) }\end{array}$ & $\begin{array}{l}102 / 136 \\
(75.0)\end{array}$ & (50/136) 36.8 & $41 / 136(30.1)$ & 109/116 (94.0) \\
\hline $\begin{array}{l}\text { HAE } \\
\text { type I } \\
\text { or II }\end{array}$ & 160 & $\begin{array}{l}99 / 160 \\
(61.9) ; \\
P<0.002 \\
\text { vs HAE } \\
\text { with normal } \\
\text { C1-INH }\end{array}$ & $\begin{array}{l}15.0 \\
(8.0-20.0) ; \\
P<0.001 \\
\text { vs } \\
\text { HAE with } \\
\text { normal } \\
\text { C1-INH }\end{array}$ & $\begin{array}{l}20.6 \\
(14.1-34.3) ; \\
P<0.001 \text { vs } \\
\text { HAE with } \\
\text { normal C1- } \\
\text { INH }\end{array}$ & $\begin{array}{l}225 \text { (64 } \\
\text { patients) }\end{array}$ & $\begin{array}{l}130 / 223 \\
(58.3)\end{array}$ & $76 / 223(34.1)$ & $\begin{array}{l}30 / 223 \\
(13.5) ; \\
P<0.001 \\
\text { vs HAE } \\
\text { with normal } \\
\text { C1-INH }\end{array}$ & 147/171 (86.0) \\
\hline
\end{tabular}


safety and effectiveness of icatibant treatment. Retrospective descriptive analyses of laryngeal HAE type I/II attacks were performed (July 09-September 13).

Results: Of 1005 icatibant-treated HAE type I/II attacks (231 patients), 67 were laryngeal (42 patients; $64.3 \%$ female): 44 $(65.7 \%)$ attacks affected the larynx only and $23(34.3 \%)$ multiple sites. Of evaluable attacks, $38 / 61(62.3 \%)$ and $23 / 61(37.7 \%)$ were treated with self- and healthcare professional-administered icatibant, respectively; $43 / 60 \quad(71.7 \%)$ were severe/very severe and $21 / 67(31.3 \%)$ were in patients on long-term prophylaxis. 6/67 (9.0\%) attacks were treated with concomitant or rescue medication. 58/66 $(87.9 \%)$ attacks were treated with one icatibant injection and $8 / 66(12.1 \%)$ with two (data missing for 1 attack). $2 / 66(3.0 \%)$ attacks were treated with one icatibant injection and used C1-inhibitor as rescue medication. Median (IQR) time to treatment was 2.0 $(1.0,8.0) \mathrm{h}$ (31 attacks), time to resolution was $6.0(2.0,21.0) \mathrm{h}$ (35 attacks), and attack duration was $8.5(4.5,19.8) \mathrm{h}(25$ attacks). For 2 evaluable attacks treated with a second injection, time between first and second injection was 3.0 and $8.0 \mathrm{~h}$; time from second injection to symptom resolution was 4.0 and $2.0 \mathrm{~h}$, respectively.

Conclusion: Most laryngeal HAE type I/II attacks were successfully treated with a single icatibant injection; a second injection or other medication was only needed in a minority of cases. The majority of attacks were treated with self-administered icatibant.

\section{8}

The Icatibant Outcome Survey: experience of hereditary angioedema management from four European countries

Caballero, $\mathrm{T}^{1,2,3} ;$ Aberer, $\mathrm{W}^{4}$; Longhurst, $\mathrm{H}^{5}$; Maurer, $\mathrm{M}^{6}$; Zanichelli, $\mathrm{A}^{7}$; Fabien, $\mathrm{V}^{8}$; Bouillet, $\mathrm{L}^{9}$; for the IOS Study Group

${ }^{1}$ Hospital La Paz Institute for Health Research (IdiPAZ), Madrid, Spain; ${ }^{2}$ Hospital Universitario La Paz, Allergy, Madrid, Spain; ${ }^{3}$ CIBERER, U754, Madrid, Spain;

${ }^{4}$ Medical University of Graz, Graz, Austria; ${ }^{5}$ Barts Health NHS Trust, London, United Kingdom; ${ }^{6}$ Charité -

Universitätsmedizin Berlin, Berlin, Germany; ${ }^{7}$ Università degli Studio di Milano, Milan, Italy; ${ }^{8}$ Shire, Eysins, Switzerland; ${ }^{9}$ Grenoble University Hospital, Grenoble, France

Background: Hereditary angioedema (HAE) is a potentially fatal, bradykininmediated disease, often misdiagnosed and undertreated, with long diagnostic delays. We compared real-world data from France (FR), Italy (IT), Spain (SP) and the UK to assess disease characteristics and treatment outcomes with icatibant, a bradykinin B2 receptor antagonist.

Method: The Icatibant Outcome Survey [IOS; Shire, Eysins, Switzerland (NCT01034969)] is an international observational study monitoring safety and effec- tiveness of icatibant. Descriptive, retrospective analyses compared IOS data (July 09-September 13) for patients (pts) with HAE type I/II in FR, IT, SP and UK.

Results: Icatibant was used to treat 738 HAE attacks in 321 pts (Table). Across countries, $57.7-63.4 \%$ of pts were female, median age at first symptoms was 9.015.0 years and at diagnosis was 17.621.8 years. Median diagnostic delay was 4.4-13.7 years, and was significantly longer in IT vs FR (Table). Annual attack frequency [median (IQR)] was higher in IT [10.2 (4.9-36.7)] and UK [12.4 (5.3-43.8)] than in FR [4.4 (0.9-10.7)] and SP [4.4 (1.8-9.7)] $73.2-86.0 \%$ of attacks in FR, IT and UK were severe/very severe, compared with only $53.8 \%$ in SP. Icatibant was selfadministered by $>50 \%$ of pts in all countries: SP $55.9 \%$; FR $76.1 \%$; IT $87.6 \%$; UK $92.0 \%$. Time to treatment was significantly less in IT and UK vs FR and SP (Table). Time to resolution was significantly longer in FR vs IT, SP or UK (Table). Attack duration was significantly longer in FR vs IT/SP/UK and in SP vs UK (Table).

Conclusion: There were long diagnostic delays in all countries. Time to treatment, time to resolution and attack duration varied between countries, perhaps indicating differing international HAE management practices.

\begin{tabular}{|c|c|c|c|c|c|c|c|}
\hline & & $\begin{array}{l}\text { France } \\
(N=160 ; \\
225 \text { Attacks) }\end{array}$ & $\begin{array}{l}\text { Italy } \\
(N=49 \\
137 \text { Attacks) }\end{array}$ & $\begin{array}{l}\text { Spain } \\
\text { (N=71; } \\
187 \text { Attacks) }\end{array}$ & $\begin{array}{l}\text { UK } \\
\text { (N=41; } \\
189 \text { Attacks) }\end{array}$ & $P$-value & \\
\hline $\begin{array}{l}\text { Delay in } \\
\text { diagnosis } \\
\text { (year) }\end{array}$ & $\begin{array}{l}\text { Mean (SD) } \\
\text { Median } \\
\text { (IOR) }\end{array}$ & $\begin{array}{c}8.7(12.7) \\
4.4(0.3-15.7)\end{array}$ & $\begin{array}{l}14.8(13.6) \\
13.7 \quad(2.0- \\
21.2)\end{array}$ & $\begin{array}{l}10.8(14.0) \\
7.7 \quad(0.8- \\
18.3)\end{array}$ & $\begin{array}{l}10.6(12.6) \\
5.1 \quad(0.0- \\
19.6)\end{array}$ & $\begin{array}{l}\text { FR vs IT } P=0.004 ; \\
\text { all others not } \\
\text { significant (NS) }\end{array}$ & \\
\hline $\begin{array}{l}\text { Time to } \\
\text { treatment } \\
\text { (h) }\end{array}$ & $\begin{array}{l}\text { Mean (SD) } \\
\text { Median } \\
(I \mathrm{QR})\end{array}$ & $\begin{array}{c}8.1(12.6) \\
3.8(1.5-9.0)\end{array}$ & $\begin{array}{l}3.0(2.9) \\
2.3(1.0-4.0)\end{array}$ & $\begin{array}{c}5.6(7.0) \\
2.3(0.8-9.2)\end{array}$ & $\begin{array}{l}3.1(5.4) \\
0.5(0.3-3.0)\end{array}$ & $\begin{array}{l}P \leq 0.02 \text { for all } \\
\text { except FR vs SP } \\
\text { (NS), IT vs UK } \\
\text { (NS) }\end{array}$ & $\begin{array}{l}\text { Only attacks with } \\
\text { complete data included. }\end{array}$ \\
\hline $\begin{array}{l}\text { Time to } \\
\text { resolution (h) }\end{array}$ & $\begin{array}{l}\text { Mean (SD) } \\
\text { Median } \\
\text { (IOR) }\end{array}$ & $\begin{array}{l}17.4(18.9) \\
12.5(1.2-24.8)\end{array}$ & $\begin{array}{c}8.0(9.9) \\
5.0(3.0-8.0)\end{array}$ & $\begin{array}{c}9.4(13.6) \\
2.6(1.0-9.7)\end{array}$ & $\begin{array}{c}7.1(9.3) \\
4.0(0.5-9.5)\end{array}$ & $\begin{array}{l}P \leq 0.02 \text { for FR vs } \\
\text { IT/SP/UK; all } \\
\text { other NS }\end{array}$ & $\begin{array}{l}\text { FR } 94 \text { attacks/31 pts IT } \\
99 \\
\text { attacks/23 pts }\end{array}$ \\
\hline $\begin{array}{l}\text { Attack } \\
\text { duration (h) }\end{array}$ & $\begin{array}{l}\text { Mean (SD) } \\
\text { Median } \\
(\text { IQR) }\end{array}$ & $\begin{array}{l}25.5(23.2) \\
18.8 \quad(10.1- \\
31.5)\end{array}$ & $\begin{array}{l}10.9(10.1) \\
8.5(5.0-13.0)\end{array}$ & $\begin{array}{l}15.0(18.1) \\
6.7 \quad(2.0- \\
21.4)\end{array}$ & $\begin{array}{l}10.2(11.3) \\
7.0 \quad(1.5- \\
15.0)\end{array}$ & $\begin{array}{l}P \leq 0.05 \text { for all } \\
\text { except IT vs UK } \\
\text { (NS) and IT vs } \\
\text { SP (NS) }\end{array}$ & $\begin{array}{l}\text { SP } 36 \text { attacks/18 pts } \\
\text { UK } 145 \text { attacks/23 pts }\end{array}$ \\
\hline
\end{tabular}




\section{Oral Abstract Session}

\section{OAS 21 - Pediatric asthma and allergy}

119

Trends in prevalence of wheeze, eczema, and hay fever in Dutch schoolchildren between 1989 and 2011

de Korte-de Boer, $\mathrm{D}^{1}$; Mommers, $\mathrm{M}^{1}$; Gielkens-

Sijstermans, $\mathrm{CML}^{2}$; Creemers, $\mathrm{HMH}^{3}$; Mujakovic, $\mathrm{S}^{4}$; Feron, $\mathrm{FJM}^{5}$; van Schayck, OCP

${ }^{1}$ Maastricht University, Epidemiology, Maastricht, The Netherlands; ${ }^{2}$ Public Health Service South Limburg, Environmental Health, Geleen, The Netherlands; ${ }^{3}$ Public Health Service South Limburg, Youth Health Care, Geleen, The Netherlands; ${ }^{4}$ Public Health Service South Limburg, Research and Development, Geleen, The Netherlands; ${ }^{5}$ Social Medicine, Maastricht University, Maastricht, The Netherlands; ${ }^{6}$ Maastricht University,

General Practice, Maastricht, The Netherlands

Background: After a worldwide rise in the prevalence of childhood asthma and wheeze, in the last years also declining trends have been observed. Underlying causes that explain these trends are largely unknown, but a role of atopy is suspected. This study aimed to investigate whether a previously observed decrease in wheeze prevalence in Dutch schoolchildren persisted, and whether prevalence trends of other atopic diseases - eczema and hay fever - showed similar patterns.

Methods: In six repeated cross-sectional surveys (in 1989, 1993, 1997, 2001, 2005, and 2010), parents of 8-11 year-old children eligible for a routine physical examination in Youth Health Care were asked to complete a questionnaire on respiratory health, including wheeze. In the last three surveys (2001-2010), ISAAC core questions on wheeze (past year), eczema (itchy flexural rash, past year) and hay fever (nasal complaints with itchy watery eyes, without a cold or the flu, past year) were introduced.

Results: Overall, participation rates of $>90 \%$ were obtained, with 1794,1526 , $1670,1686,1467$, and 704 participants in the surveys respectively. After an initially decreasing trend in wheeze from 1989 to 2001, wheeze prevalence did not further decrease between 2001 and $2010(7.2 \%$ in $2001,8.2 \%$ in 2005 , and $6.0 \%$ in 2010 ; $\mathrm{p}_{\text {trend }}>0.05$ ). The prevalence of eczema did not change significantly between 2001 and $2010(11.7 \%$ in $2001,11.9 \%$ in 2005 , and $10.6 \%$ in $2010 ; p_{\text {trend }}>0.05$ ), but hay fever prevalence increased significantly from $8.4 \%$ in 2001 to $11.8 \%$ in 2010 ( $\mathrm{p}_{\text {trend }}$ $<0.01)$.
Conclusion: After a decreasing trend of wheeze prevalence among Dutch 811 year-old children between 1989 and 2001, no further decrease was observed between 2001 and 2010. The prevalence of eczema remained stable between 2001 and 2010, while hay fever prevalence showed an increasing trend in this period. Finding explanations for these diverging trends forms a starting point for further unravelling the underlying causes of atopic diseases.

\section{0 \\ Sensitisation to dog allergen components from childhood to adolescence in relation to dog allergy: a MeDALL project}

Asarnoj, $A^{1,2}$; Wadén, $K^{1}$; Hamsten, $C^{1}$; Andersson, $N^{3}$; Lupinek, $\mathrm{C}^{4}$; Anto, $\mathrm{J}^{5}$; Bousquet, $\mathrm{J}^{6}$; Valenta, $\mathrm{R}^{4}$; van Hage, $\mathrm{M}^{1}$; Wickman, $\mathrm{M}^{3,7}$

${ }^{1}$ Clin Immunology and Allergy Unit, Department of Medicine, Karolinska Institutet, Stockholm, Sweden; ${ }^{2}$ Department of Pediatric Pulmonology and Allergy, Karolinska University Hospital, Astrid Lindgren Children's Hospital, Stockholm, Sweden; ${ }^{3}$ Karolinska Institutet, National Institute of Environmental Medicine, Stockholm, Sweden; ${ }^{4}$ Division of Immunopathology, Department of Pathophysiology and Allergy Research, Center for Pathophysiology, Infectiology and Immunology, Medical University of Vienna, Vienna, Austria; ${ }^{5}$ Departament de Ciències Experimentals i de la Salut, Centre for Research in Environmental Epidemiology (CREAL), IMIM (Hospital del Mar Research Institute), CIBER Epidemiología y Salud Pública (CIBERESP), Universitat Pompeu Fabra, Barcelona, Spain; ${ }^{6}$ Hôpital Arnaud de Villeneuve, University Hospital of Montpellier, Montpellier, INSERM 1018, Villejuif, France; ${ }^{7}$ Södersjukhuset, Sachs' Children's Hospital, Stockholm, Sweden

Background: Allergy to dog (Canis familiaris) is common and different dog allergen components (DAC) may contribute differently to development of dog allergy (DA). Our aim was to investigate the sensitisation pattern to the various DAC from childhood to adolescence in relation to DA at 16 years.

Method: Sera and questionnaire data from 786 randomly collected children of a population based birth cohort (BAMSE) were analyzed at age 4,8 and 16 years. DA at the same ages was defined as reported rhinitis, conjunctivitis or asthma triggered by dog contact. IgE to the DAC Can f 1, 2, 3, 5 and 6 were analyzed with an allergen chip based on ISAC technology (Thermo
Fisher) developed in the MeDALL FP7funded research program.

Results: $8.7 \%$ of the children reported DA at least at one follow up. IgE to any of the DAC was found in $8.1 \%$ at 8 and $14.1 \%$ at 16 years. Sensitisation to Can $\mathrm{f} 1$ at 8 years increased the risk for DA at age 16 (OR adjusted for sensitisation to other DAC: $13,95 \%$ CI 4.1-41.1), of whom $35 \%$ had been persistent from 8 years. Analysis of DAC sensitisation and DA at the same age showed that children sensitised to Can $\mathrm{f} 1$ and not to Can $\mathrm{f} 5$ significantly more often reported DA, compared to children sensitised to Can $\mathrm{f} 5$ and not to Can f 1 , both at $8(P<0.01)$ and 16 $(P=0.04)$ years. A total of $12 \%$ were without dog symptoms but sensitised to at least one DAC at some time point ever. Most of them had IgE to Can $\mathrm{f} 5(85 \%)$. Dog ownership at any time point was not associated with sensitisation to any DAC. No association was found between IgE reactivity to Can $\mathrm{f} 3$ and reported DA.

Conclusion: Our results suggest that sensitisation to the dog allergen component Can $\mathrm{f}$ 1 is the most important marker of DA. IgE reactivity to Can $\mathrm{f} 5$ is associated to DA, but not to the same extent as $\operatorname{Can} \mathrm{f} 1$. IgE to Can $\mathrm{f} 3$ seems to have limited or no importance for symptoms to dog at exposure.

121

Sensitisation patterns in childhood in the COPSAC $_{2000}$ high-risk birth cohort study

Schoos, A-MM ${ }^{1,2}$; Rasmussen, MA ${ }^{1}$; Chawes, BLK ${ }^{1}$; Bønnelykke, $\mathrm{K}^{1}$; Bisgaard, $\mathrm{H}^{1}$

'Danish Pediatric Asthma Center, Gentofte, Denmark ${ }^{2}$ Department of Pediatrics, Mount Sinai School of Medicine, New York, NY, United States

Background: Sensitisation to a specific allergen during childhood rarely appears alone and gives limited information about development of symptomatic disease. Thus we propose, that analyzing longitudinal measurements of sensitisation, including age at onset, development over time and the number of allergens involved, may reveal patterns of sensitisation that are closer associated to clinical outcomes.

Method: 411 children were included from the COPSAC $_{2000}$ birth cohort from whom specific IgE measurements were assessed 
against 13 common food- and inhalant allergens at ages $1 \frac{1}{2}, 1 \frac{1}{2}, 4$ and 6 years. We used an unsupervised data driven clustering technique to extract a set of latent patterns that characterise development of sensitisation during preschool age. Subsequently, these patterns were investigated in relation to development of asthma, rhinitis and atopic dermatitis.

Results: The pattern analysis indicated a complex latent structure involving a total of 7 age- and allergen-specific patterns: (i) dog/cat/horse; (ii) grass/birch; (iii) molds; (iv) house dust mites; (v) peanut/wheat flour/mugwort; (vi) peanut/soybean; (vii) egg/milk/wheat flour. Patterns 1-5 were mainly influenced by sensitisation at ages 4 and 6 years while the food patterns ( 6 and 7) where influenced by sensitisation also from the earliest age points. Asthma was associated to pattern 1 only $[\mathrm{OR}=3.3$ $(1.5 ; 7.2)]$, rhinitis to patterns $1,2,3,4$ and $6(\mathrm{ORs}=2.2-4.3)$ and atopic dermatitis to patterns 1, 2, 3, 5, 6 and $7(\mathrm{ORs}=1.6-$ 2.5).

Conclusion: This study proposes the presence of separate sensitisation patterns differentially associated to development of clinical outcomes.

122

Single-breath FeNO measurement in preschool children using a new method and device

Kalm-Stephens, $\mathrm{P}^{1}$; Heijkenskjöld Rentzhog, $\mathrm{C}^{1}$; Nordvall, $\mathrm{L}^{1}$; Malinovschi, $\mathrm{A}^{2}$; Alving, $\mathrm{K}^{1}$

'Department of Women's and Children's Health, Uppsala University, Uppsala, Sweden; ${ }^{2}$ Department of Medical Sciences, Uppsala University, Uppsala، Sweden

Background: Measurement of the fraction of exhaled nitric oxide (FeNO) is an established method for monitoring airway inflammation. However, measurement in children younger than six years remains a challenge. The aim of this study was to evaluate the feasibility of single-breath FeNO measurement in children using a new method and device adapted for young children, and to confirm that clinically reasonable signals are provided with the new procedure.

Method: FeNO measurements were performed in a total of 62 children 3-10 years of age, 53 children with asthma (31 on inhaled corticosteroid (ICS) treatment) and 9 non-allergic controls. The children exhaled at a target flow rate of $50 \mathrm{ml} / \mathrm{s}$ into a new prototype electrochemical device (NIOX NOVA; Aerocrine), and air was simultaneously fed into a fast-response analyser (NIOX Flex) for evaluation of time to NO plateau. A plateau of at least $2 \mathrm{~s}$ was identified by using a computer algorithm. The method allowed higher pressures than the usual limit of $20 \mathrm{~cm}$ $\mathrm{H}_{2} 0$, while still keeping target flow rate, and exhalation time was preliminary adjusted for age for children 3-8 years (4$8 \mathrm{~s}$ ).

Results: Five of $12(42 \%)$ of the 3-year olds, $19 / 20(95 \%)$ of the 4-6 year olds, and $29 / 30(97 \%)$ of the $7-10$ year olds were able to perform at least one acceptable measurement. The time to NO plateau was correlated with body height and absolute FeNO level and ranged between 3-6 s for children 3-8 years of age. FeNO values are presented in the table below.

\begin{tabular}{|c|c|c|c|}
\hline $\begin{array}{l}\text { FeNO } \\
(\mathrm{ppb}) \\
{[\mathrm{GM}} \\
(95 \% \mathrm{Cl})]\end{array}$ & $\begin{array}{l}\text { Non- } \\
\text { Allergic } \\
\text { Controls }\end{array}$ & $\begin{array}{l}\text { ICS- } \\
\text { Naive } \\
\text { Asthma }\end{array}$ & $\begin{array}{l}\text { ICS-Treated } \\
\text { Asthma }\end{array}$ \\
\hline $\begin{array}{l}\text { Children } \\
3- \\
10 \text { years }\end{array}$ & $\begin{array}{l}7.0 \\
(5.2- \\
9.5)^{*} \\
(n=8)\end{array}$ & $\begin{array}{l}16.4 \\
(12.2- \\
22.2) \\
(n=19)\end{array}$ & $\begin{array}{l}9.0(6.9-11.7)^{*} \\
(n=25)\end{array}$ \\
\hline $\begin{array}{l}\text { Children } \\
3- \\
6 \text { years }\end{array}$ & $\begin{array}{l}6.6 \\
(3.7- \\
11.8)^{* *} \\
(n=5)\end{array}$ & $\begin{array}{l}15.2 \\
(9.3- \\
24.7) \\
(n=6)\end{array}$ & $\begin{array}{l}7.1 \\
(4.5-11.2)^{* *} \\
(n=11)\end{array}$ \\
\hline
\end{tabular}

Conclusion: This study shows that children as young as three years old can perform acceptable single-breath FeNO measurements with the modified method based on more robust flow control and shortened exhalation times. Time to NO plateau was less than $6 \mathrm{~s}$ for ages 3-8 years, which confirms that the exhalation time can be markedly shortened for this age group. A clearcut clinical signal was shown using the new method and device, even though FeNO values were commonly below $20 \mathrm{ppb}$ in young children with asthma.

\section{3 \\ Which patterns of allergen sensitisation identify an increased risk for asthma in children with atopic dermatitis?}

Calamelli, $E^{1} ;$ Ricci, $L^{2}$; Rondelli, $\mathrm{R}^{1}$; Ricci, $\mathrm{G}^{1}$; Pession, $\mathrm{A}^{1} ;$ Patrizi, $\mathrm{A}^{2}$

${ }^{1}$ Pediatric Unit, Department of Medical and Surgical Sciences, University of Bologna, Bologna, Italy; ${ }^{2}$ Dermatology Unit, Department of Specialistic, Diagnostic and Experimental Medicine, University of Bologna, Bologna, Italy

Background: Atopic dermatitis (AD) is a chronic inflammatory skin disorder affecting mostly young children. Over the last decades, it has been strengthened the socalled 'atopic march' theory, defined as the natural progression from AD towards the development of respiratory allergy. Although longitudinal studies aimed to identify the possible risk factors for asthma, several aspects of this phenomenon still need to be clarified. The aim of this study is to evaluate the natural course of inhalant and food allergen sensitisation in children with $\mathrm{AD}$ and its relation to asthma.

Method: We enrolled 99 children with IgE-mediated $\mathrm{AD}$ referred to the Pediatric Dermatology and Pediatric Allergology Units of Bologna University. All children performed clinical evaluation and total and specific $\operatorname{IgE}$ assay for the most common classes of inhalant and food allergens (grasses, house dust mite, cat dander, cow's milk and egg white) at 2 different times (t) during pre-school age (t0, mean age $15 \pm 3$ months and t1, mean age $30 \pm 3$ months old) and then at intermittent times according to the clinical needs (mean age of follow-up 10 years). Asthma diagnosis was assessed at one follow-up visit performed during school-age. Univariate and multivariate logistic regression were used to determine the risk of asthma. Results: At follow-up visit, 39\% of children had developed asthma. Of the variables compared, the presence of sensitisation to more than one class of inhalant allergens at $\mathrm{t} 1$ was associated with asthma $(P<0.005)$. The multivariate analysis demonstrated that grass pollen $[\mathrm{OR}=3.2$ (95\% CI: 1.2, 8.7), $P<0.05]$ and cat sensitisation $[\mathrm{OR}=2.7 \quad(95 \% \quad \mathrm{CI}: \quad 1.1, \quad 7.3)$, $P<0.05$ ] were independent risk factors for asthma. No relation was found between asthma and early food allergen sensitisation.

Conclusion: Our data showed that children with AD who develop sensitisation to multiple classes of aeroallergens during early preschool (mean age 30 months old) are at increased risk for asthma during school age.

\section{4 \\ The effect of prednisolone in the first rhinovirus-induced wheeze: a randomised controlled trial with 12-month follow-up}

Jartti, $\mathrm{T}^{1}$; Nieminen, $\mathrm{R}^{1}$; Vuorinen, $\mathrm{T}^{2}$; Lehtinen, $\mathrm{P}^{1}$; Vahlberg, $\mathrm{T}^{3}$; Gern, $\mathrm{J}^{4}$; Camargo, CA Jr ${ }^{5}$; Ruuskanen, $\mathrm{O}^{1}$ ${ }^{1}$ Turku University Hospital, Pediatrics, Turku, Finland; ${ }^{2}$ University of Turku, Virology, Turku, Finland;

${ }^{3}$ University of Turku, Biostatistics, Turku, Finland; ${ }^{4}$ Pediatrics and Medicine, University of Wisconsin School of Medicine and Public Health, Madison, United States; ${ }^{5}$ Division of Rheumatology, Allergy and Immunology, Emergency Medicine and Medicine, Massachusetts General Hospital, Harvard Medical School, Boston, MA, United States

Background: Rhinovirus-induced wheeze is an important risk factor for recurrent wheezing of childhood. To date, there are no randomised controlled trials on the 
effect of systemic corticosteroids in rhinovirus-induced wheeze. Our objective was to study the short-term and long-term effects of prednisolone treatment of the first rhinovirus-induced wheeze in young children.

Method: After confirming rhinovirus from nasopharyngeal aspirate by PCR, 79 children with first wheeze, aged 3-23 months, were randomised to receive oral prednisolone (first dose $2 \mathrm{mg} / \mathrm{kg}$, then $2 \mathrm{mg} / \mathrm{kg} /$ day in 2 divided doses for 3 days) or placebo. The trial was double-blinded throughout the 12-month follow-up. The primary outcomes were long-term: new physician-confirmed wheezy episode within 2 months, number of physician-confirmed wheezy episodes within 12 months, and initiation of regular controller medication for asthma symptoms within 12 months. The primary interaction analysis examined rhinovirus load.

Results: Seventy-four patients completed the study (mean age 13 months; $28 \%$ atopic). Long-term outcomes did not differ between groups (all $P \geq 0.30$ ). In shortterm outcomes, the prednisolone group had less cough, rhinitis, noisy breathing, severe breathing difficulties and nocturnal respiratory symptoms at home within 2 weeks (all $P<0.05$ ). The 20 children with $\geq 7000$ rhinovirus copies $/ \mathrm{ml}$ benefitted from prednisolone in terms of less risk of physician-confirmed recurrence within 2 months and less physician-confirmed wheezy episodes within 12 months (both $P<0.05)$.

Conclusion: Despite some short-term benefits, prednisolone cannot be routinely recommended for the first rhinovirus-induced wheeze in young children. The link between rhinovirus load and long-term effect of prednisolone warrants further study. 


\section{Oral Abstract Session}

\section{OAS 22 - Mast cells and basophils}

\section{5 \\ The biological clock controls the functionality of human intestinal mast cells}

Baumann, A; Bischoff, SC; Lorentz, A

Institute of Nutritional Medicine, University of

Hohenheim, Stuttgart, Germany

Background: Allergic diseases are frequently exacerbated between midnight and the early morning suggesting an involvement of the biological clock. We have recently shown that mast cells - known as major inflammatory effector cells of allergic diseases - express an intact circadian clock. All examined clock genes hPerl, hPer2, hCryl, hBmall and hClock are expressed and exhibit circadian rhythm in human intestinal mast cells (hiMC). Here, we analyzed the role of the biological clock in the functionality of hiMC.

Method: HiMC were isolated from intestinal mucosa by combined mechanic and enzymatic tissue dispersion and purified using magnetic cell separation system. Long-term cultured hiMC were synchronized by addition of $40 \mu \mathrm{M}$ dexamethasone for $2 \mathrm{~h}$ to reset the biological clock. Then, hiMC were activated by FceRI crosslinking using $\operatorname{IgE} / \operatorname{anti}-\operatorname{IgE}$ every $4 \mathrm{~h}$ around the circadian cycle. Clock gene mRNA expression was analyzed by real-time RT-PCR, the release of histamine and cysteinyl leukotrienes (cysLTs) was measured by ELISA.

Results: All the analyzed clock genes hPer1, hPer 2, hCry1, hBmal1, and hClock were expressed in a similar circadian pattern in IgE-stimulated hiMC as in unstimulated cells. Most interestingly, circadian oscillation could be detected for the release of pre-stored histamine as well as of de novo synthesized cysLTs in response to FceRI cross-linking in hiMC. The highest release of both mediators was found at time point $20 \mathrm{~h}$.

Conclusion: In summary, the circadian clock is involved in the functionality of hiMC leading to circadian production and release of their mediators. Thus, the biological clock may play a pivotal role in the pathophysiology of allergy and could be of relevance as a target in allergic diseases.

\section{6 \\ Novel anti-inflammatory function of alpha-melanocyte stimulating hormone on human basophils}

Kleiner, $\mathrm{S}^{1}$; Braunstahl, G-J $\mathrm{J}^{2}$; Gehring, $\mathrm{M}^{1}$; Eiz-Vesper, $\mathrm{B}^{3}$; Luger, $\mathrm{T}^{4}$; Kapp, $\mathrm{A}^{1}$; Böhm, $\mathrm{M}^{4}$; Raap, $\mathrm{U}^{1}$ ${ }^{1}$ Department of Dermatology and Allergy, Hannover Medical School, Hannover, Germany; ${ }^{2}$ Department of Pulmonology, Sint Franciscus Gasthuis, Rotterdam, The Netherlands; ${ }^{3}$ Institute for Transfusion Medicine, Hannover Medical School, Hannover, Germany; ${ }^{4}$ Department of Dermatology and Allergy, University of Münster, Münster, Germany

Background: $\alpha$-melanocyte-stimulating hormone $(\alpha-\mathrm{MSH})$ was shown to exert antiallergic effects in human basophils.

Objective: This study aims to extend our current knowledge about the effects of $\alpha$ MSH on basophils especially from patients with allergic rhinitis (AR).

Methods: We included three different study groups. In the first group we analyzed nasal tissue of AR patients and healthy controls $(n=9$ each $)$, in the second group whole blood basophils of AR patients $(n=14)$ and healthy controls $(n=19)$. In the third group we investigated isolated basophils derived from healthy controls $(n=13)$. Tissue expression before and after nasal allergen provocation and in vitro inducibility of melanocortin receptor-1 (MC-1R) was analyzed using immunofluorescent staining, flow cytometry and qRT-PCR. Basophil activity was evaluated by measurement of CD203c, cytokine release and chemotaxis. Viability was analysed by means of propidiumiodid/AnnexinV and Jc-1 staining.

Results: MC-1R positive basophils were increased in nasal mucosa tissue of AR patients $24 \mathrm{~h}$ after nasal allergen challenge. MC-1R expression was significantly higher in blood basophils of patients with AR compared to healthy controls. MC-1R expression was inducible on whole blood basophils by different stimuli including fMLP and anti-IgE. $\alpha$-MSH inhibited anti$\mathrm{IgE}$ and grass pollen induced upregulation of the clinically relevant activation marker CD203c. $\alpha$-MSH functioned as a chemoattractant and increased viability in isolated basophils. Moreover, $\alpha$-MSH induced the release of the anti-inflammatory cytokine IL-10.

Conclusion: In AR nasal allergen provocation enhances influx of MC-1R positive basophils. Additionally, $\alpha$-MSH triggers an anti-inflammatory activation pattern in human basophils with inhibition of CD203c and release of IL-10.

\section{7 \\ The novel flavone tetramethoxyluteolin potently inhibits human mast cell mediator release \\ Weng, Z; Theoharides, TC Department of Integrative Physiology and Pathobiol, Tufts University School of Medicine, Boston, MA, United States}

Background: Mast cells (MC) are hemopoietic cells that mature in tissues and are involved in allergy, immunity and inflammation by secreting multiple mediators. Currently, there is no effective MC inhibitor as the action of disodium cromoglycate (cromolyn) has been questioned. The natural flavone luteolin (lut) has antioxidant and anti-inflammatory actions, but its effect on MC has not been fully investigated. We will investigate the ability of lut, and its novel structural analog $3^{\prime}, 4^{\prime}, 5,7$-tetramethoxyluteolin (methlut), to inhibit human $\mathrm{MC}$ activation and mediator release.

Method: Human LAD2 MC were stimulated with substance $\mathrm{P}$ (SP) and primary human umbilical cord-blood derived cultured MC (hCBMCs) with IgE/anti-IgE. MC mediator release with or without lut or methlut $(1-100 \mu \mathrm{M})$ preincubation was measured by ELISA. Gene expression was determined by qRT-PCR. The effect of lut and methlut on intracellular calcium levels and NF- $\kappa \mathrm{B}$ activation was also investigated.

Results: Activated human MC rapidly release beta-hexosaminidase ( $\beta$-hex), histamine, prostaglandin $\mathrm{D}_{2}\left(\mathrm{PGD}_{2}\right)$, and preformed tumor necrosis factor (TNF). MC also synthesize and secrete TNF and chemokine (C-C motif) ligand 2 (CCL2) several hours later. Preincubation with lut or methlut dose-dependently decreases release of all mediators without reducing intracellular ATP production. Lut and methlut block intracellular calcium increase and decrease $\mathrm{NF}-\kappa \mathrm{B}$ induction at both the transcriptional and translational levels. Methlut is more effective than lut in every aspect examined. 
Conclusion: Methlut is a promising MC inhibitor for the treatment of allergic and inflammatory conditions where MC activation is involved.

\section{8 \\ The succinate-GPR91 axis is involved in the regulation of mast cell response}

Chang, $\mathrm{H}-\mathrm{W}^{1}$; Kawasaki, $\mathrm{H}^{1}$; Tseng, $\mathrm{H}^{-} \mathrm{C}^{1}$; Hsu, $\mathrm{S}^{-\mathrm{C}^{1}}$; Yang, S-J ${ }^{1} ;$ Huang, S-K ${ }^{1,2}$

${ }^{1}$ Division of Environmental Health and Occupational Medicine, National Health Research Institutes, Miaoli County, Taiwan; ${ }^{2}$ Johns Hopkins University School of Medicine, Baltimore, MD, United States

Background: Succinate, an intermediate of the TCA cycle in the mitochondria, has recently been shown to regulate various cellular functions through the G-proteincoupled, metabolic receptor, GPR91. But, its role in the regulation of innate immunity and mast cells, a critical cell type in mucosal and allergic responses, remain unclear. The objective of this study was, therefore, to investigate whether the succinate-GPR91 axis plays a role in regulating the mast cell response.

Methods: Mouse bone marrow-derived mast cells (BMMCs) and human peripheral blood-derived cultured mast cells (HCMCs) were used as a model, and the effect of varying doses of succinate on $\mathrm{IgE}$ mediated mast cell response was examined. Degranulation was measured by analyzing the level of b-hexosaminidase, and the levels of lipid mediator release and cytokine production were analyzed by ELISA. Flow cytometric analyses were performed to determine the expression of GPR91 and the level of intracellular calcium, while Western blotting was used to determine the level of ERK activation.

Results: Flow cytometric analysis showed first that mast cells expressed, constitutively, GPR91, and its level increased in activated mast cells. Significantly, increased levels of degranulation, leukotriene $\mathrm{C} 4$ (LTC4) and IL-13 were noted in succinatetreated BMMCs and HCMCs following the IgE-mediated activation, concomitant with enhanced levels of intracellular calcium and phosphorylated ERK. Interestingly, succinate appeared to be able to inhibit the release of prostaglandin E2 (PGE2), which was in contrast to the increased level of LTC4, in activated mast cells, suggesting a differential impact of succinate on the generation of lipid mediators.

Conclusions: These results suggest that the succinate-GPR91 axis is functional in regulating the responses of both human and mouse mast cells, supporting the existence of a novel receptor-ligand axis in controlling mast cell and hence the allergic response.

\section{9}

Inhibition of mast cell activation by short chain fatty acids

Folkerts, $\mathrm{J}^{1,2}$; Redegeld, $\mathrm{F}^{1}$; Schmetzer, $\mathrm{O}^{2}$; Maurer, $\mathrm{M}^{2} ;$ Folkerts, $\mathrm{G}^{1}$

${ }^{1}$ Department of Pharmaceutical Sciences, Utrecht Institute for Pharmaceutical Sciences, Utrecht University, Utrecht, The Netherlands; ${ }^{2}$ Department of Dermatology and Allergy, Charité -

Universitätsmedizin, Berlin, Germany

Introduction: The fermentation of fibers by bacteria in the gut results in the production of Short Chain Fatty Acids (SCFAs) such as acetate, butyrate and propionate. SCFAs are believed to be critically important for immunoregulation. Previous studies showed that GPR41 and GPR43, both receptors for SCFA, were expressed on mucosal mast cells in the rat intestine. In this study, we assessed the functional effects of the three most prevalent SCFAs, acetate, butyrate, and propionate on mast cell activation.

Methods: Bone marrow derived mast cells where cocultured with SCFAs for $24 \mathrm{~h}$ (1, 5 and $25 \mathrm{mM}$ ) or non-SCFA containing medium before activation. Mast cell degranulation was assayed by the release of beta-hexosaminidase activity. As a typical late phase reaction of mast cells to allergen challenge, IL-6 production was assessed. Viability of the cells was measured using an LDH-kit (Roche Diagnostics).

Results: All three SCFAs tested (acetate, butyrate, and propionate) potently inhibited mast cell activation in a dose-dependent manner. Pretreatment with acetate, butyrate and propionate concentrations of 5 and $25 \mathrm{mM}$ gave a $70-95 \%$ inhibition of IgE-mediated mast cells degranulation $(P<0.01$ vs control). SCFAs also inhibited IL-6 synthesis (reduction of $81 \%, 99 \%$ and $92 \%$ respectively, $P<0.01)$. SCFA treatment also affected mast cell degranulation caused by the calcium ionophore ionomycin (percentage inhibition beta-hexosaminidase release: acetate: $32 \%, \quad P<0.05$; butyrate $46 \%, P<0.05$; and propionate $36 \%, P<0.01)$. Toxicity of each of the SCFAs was assessed to rule out the possibility of inhibited mast cell responses due to cell death. Both acetate and propionate had no toxic effects on mast cells as assessed by LDH leakage. Butyrate induced only a minor increase in cell death at concentrations of $5 \mathrm{mM}$ and higher.

Conclusions: We show that SCFAs potently inhibit allergic mast cell activation resulting a decreased degranulation and cytokine production. Effective inhibitory concentrations of acetate, butyrate and propionate are within physiological ranges of SCFAs found e.g. gut (up to $200 \mathrm{mM}$ ) and blood (up to $20 \mathrm{mM}$ ), which suggests a potential physiological relevance of bacterial fermentation products in modulation of allergic mast cell activation. Future research will be directed to further investigate the involvement of GPR41/43 in modulation of mast cell activation by SCFAs.

\section{0 \\ Human basophils modulate monocyte activation}

Rivellese, $\mathrm{F}^{1,2}$; Suurmond, $\mathrm{J}^{1}$; de Paulis, $\mathrm{A}^{2}$; Marone, $\mathrm{G}^{2}$; Huizinga, TW ${ }^{1}$; Toes, REM ${ }^{1}$

${ }^{1}$ Department of Rheumatology, Leiden University Medical Center, Leiden, The Netherlands; ${ }^{2}$ Division of Allergy and Clinical Immunology and Center for Basic and Clinical Immunology Research (CISI), University of Naples Federico II, Naples, Italy

Background: Basophils are circulating granulocytes, classically known as effector cells in allergic reactions. According to recent experimental models of allergic and autoimmune inflammation in mice, they may also act as immunoregulatory cells in vivo, mainly through the production of IL4, which was shown to direct monocyte activity and differentiation toward an antiinflammatory phenotype. Nonetheless, it is not known whether human basophils are also capable of driving immunoregulatory responses. Therefore, aim of this study was to analyse the influence of human basophils on monocyte activation.

Methods: Human basophils and monocytes were isolated from buffy coats of healthy donors. Basophils were activated with different combinations of cross-linking antiIgE antibody and IL-33. Monocytes were conditioned with the supernatants of activated basophils or co-cultured with basophils and LPS-induced activation was evaluated by measuring cytokine production by ELISA and by flow cytometric analysis of surface markers.

Results: Human basophils activated via IL33 and IgE cross-linking significantly suppressed the LPS-induced production of the prototypical pro-inflammatory cytokine TNF-alpha and the upregulation of the costimulatory molecule CD80 by monocytes. These effects were mainly explained by the production of histamine, as they could be inhibited by the histamine receptor 2 (H2R) antagonist ranitidine, with a small contribution of basophil-derived IL-4. In contrast, basophil-derived IL-4 and histamine had opposing effects on the expression of the inhibitory Fc gamma receptor IIb or the production of IL-10 by monocytes.

Conclusion: Our results show how human basophils influence monocyte activation. These data suggest a previously unrecognised role for human basophils, through the balanced production of histamine and IL4 , in the regulation of the immune response, with potential implications in the pathogenesis of allergic diseases. 


\section{Oral Abstract Session}

\section{OAS 23 - Biomarkers in asthma}

\begin{abstract}
131
Blood-based molecular biomarkers of allergen-induced early and late asthmatic responses

Tebbutt, SJ ${ }^{1,2}$; Singh, $A^{2,3}$; Shannon, $C^{2}$; Gauvreau, ${\overline{\mathrm{GM}^{4}}}^{4}$ Fitzgerald, $\mathrm{M}^{1}$; Boulet, L-P $\mathrm{P}^{5}$; O'Byrne, $\mathrm{PM}^{4}$ ${ }^{1}$ University of British Columbia, Medicine, Vancouver, BC, Canada; ${ }^{2}$ PROOF Centre of Excellence, Vancouver, BC, Canada; ${ }^{3}$ University of British Columbia, Vancouver, BC, Canada; ${ }^{4}$ McMaster University, Medicine, Hamilton, ON, Canada; ${ }^{5}$ Laval University, Quebec City, QC, Canada
\end{abstract}

Background: Asthma is a chronic condition affecting 300 million people worldwide, and remains poorly understood due to its complexity and heterogeneity. Two particularly important subtypes of asthma can be identified via a human experimental model of allergen exposure in which sensitised individuals undergo allergen inhalation challenge (AIC). Individuals with allergic asthma respond differently, but reproducibly, to this allergen challenge. Some develop an isolated early response (ER) while others also go on to develop a late response (dual responders; DR). It is not understood why late responses do not develop in all sensitised subjects. Since it is the consequences of the late asthmatic response that are believed to contribute to chronic airway inflammation and uncontrolled disease it is important to identify molecular mechanisms and biomarkers that can discriminate between these different response types.

Method: We profiled the peripheral whole blood transcriptomes of 17 ER individuals and 18 DR individuals, both pre-AIC and 2-3 h post-AIC. Illumina rRNA/globindepleted stranded cDNA libraries were generated from total cellular RNA, and paired-end HiSeq sequencing was performed on each library.

Results: Approximately 26 million paired reads were obtained per sample. Differential expression analysis pre-AIC revealed 464 differentially expressed RefSeq transcripts at an FDR of 10\% (165 over- and 299 under-expressed in DRs). Top MetaCore pathways (FDR $<0.1 \%$ ) included: Blood coagulation_GPCRs in platelet aggregation \& Cell adhesion_Integrin inside-out signaling. Post-AIC, 32 (15 overand 17 under-expressed in DRs) differentially expressed RefSeq transcripts were identified, with the top MetaCore pathway
(FDR $<1 \%$ ) being Immune response_Antigen presentation by MHC class I. Preliminary biomarker discovery and crossvalidation using elastic net classifiers demonstrated a mean AUC (area under the receiver operating characteristic (ROC) curve) of $\sim 70 \%$ for discriminating ER from DR at pre-AIC.

Conclusion: Allergen inhalation challenge can be a suitable experimental model to identify biomarkers that can discriminate between early and late asthmatic response types and to help elucidate the mechanism of allergic asthma in humans.

\section{2}

Associations of serum periostin level with AERD phenotype and clinical parameters

Kim, M-A ${ }^{1}$; Izuhara, $\mathrm{K}^{2}$; Ohta, $\mathrm{S}^{2}$; Ono, $\mathrm{J}^{3} ;$ Yoon, $\mathrm{MK}^{4}$; $\overline{\mathrm{Kim}, \mathrm{JH}^{4}}$; Ban, $\mathrm{GY}^{4}$; Yoo, H-S ${ }^{4}$; Shin, $\mathrm{YS}^{4}$; Ye, Y-M ${ }^{4}$; Nahm, D- ${ }^{4}$; Park, $\mathrm{H}^{-\mathrm{S}^{4}}$

${ }^{1}$ Department of Allergy and Clinical Immunology, $\mathrm{CHA}$ Bundang Medical Center, CHA University, Suwon, Korea; ${ }^{2}$ Saga Medical School, Saga, Japan; ${ }^{3}$ ShinoTest Corporation, Kanagawa, Japan; ${ }^{4}$ Department of Allergy and Clinical Immunology, Ajou University Hospital, Suwon, Korea

Background: Recent studies suggest periostin as a systemic biomarker of eosinophilic airway inflammation to predict responses to novel treatments that targets eosinophilic Th2-driven inflammation in asthmatic patients. We investigated the clinical implications of serum periostin levels in aspirin-exacerbated respiratory disease (AERD) patients based on an overlapping Th2-mediated pathogenesis with eosinophilic asthma.

Method: Serum periostin levels were measured by human periostin enzyme-linked immunosorbent assay (ELISA) in sera from 277 adult asthma patients. Serum periostin levels were compared between AERD and aspirin tolerant asthma (ATA) with other asthma phenotypes such as severe/non-severe asthma and eosinophilic/ non-eosinophilic asthma. The phenotype of AERD and ATA was classified according to the results of a lysine-aspirin bronchoprovocation test (Lys-ASA BPT). The association of serum periostin levels with clinical parameters (including disease severity and comorbid condition) was analyzed.
Results: Serum periostin levels were significantly higher in AERD vs ATA as well as in severe asthma vs non-severe asthma and eosinophilic asthma vs non-eosinophilic asthma $(P=0.002, P=0.020, P<0.001$, respectively). Multivariate regression analysis demonstrated serum periostin levels as a significant parameter to predict AERD phenotype $(P=0.003)$. In addition, serum periostin levels correlated with blood eosinophil counts $(r=0.252, \quad P<0.001)$ and sputum eosinophil count $(r=0.230$, $P=0.002)$. Higher serum periostin levels were noted in AERD patients comorbid of more severe chronic rhinosinusitis (CRS) (Lund-Mackay (LM) stage 3, 4) than those with less severe CRS (LM stage 1, 2) $(P=0.027)$. In addition, a significant positive correlation between the serum periostin level and percent fall of FEV1 after a Lys-ASA BPT was noted in AERD patients $(P=0.021)$.

Conclusion: Serum periostin can be a novel serum biomarker candidate to discriminate AERD phenotype from ATA.

\section{3 \\ Unsupervised cluster analysis of sputum cytokine profiles in a large asthma patient cohort \\ Seys, $\mathrm{S}^{1}$; Scheers, $\mathrm{H}^{2}$; Marijsse, $\mathrm{G}^{1}$; Dilissen, $\mathrm{E}^{1}$; Van den Bergh, $A^{3}$; Goeminne, $P^{2}$; Van den Brande, $P^{3}$; Ceuppens, $\mathrm{JL}^{1}$; Dupont, $\mathrm{LJ}^{2}$; Bullens, $\mathrm{DM}^{4}$ \\ ${ }^{1} \mathrm{KU}$ Leuven, Clinical Immunology, Leuven, Belgium; ${ }^{2}$ KU Leuven, Pulmonology, Leuven, Belgium; ${ }^{3} \mathrm{UZ}$ Leuven, Pulmonology, Leuven, Belgium; ${ }^{4} \mathrm{KU}$ Leuven, Pediatric Immunology, Leuven, Belgium}

Background: Asthma patients have a heterogeneous inflammatory profile, which has been linked previously with the response to inhaled steroids.

Objective: To identify unique patient clusters with a selective airway cytokine expression ('cytokine-high') profile in an unselected patient population with asthma. Method: Induced sputum samples were analysed from 208 asthma patients and 80 healthy individuals. Medical history, asthma symptoms and control questionnaires, lung function parameters as well as current treatment were recorded. Patients were defined as being 'cytokine-high' if sputum mRNA expression levels of a particular cytokine exceeded the 90th 
percentile value of the control group. An unsupervised hierarchical cluster approach was used to determine patient clusters based on sputum cytokine profiles.

Results: Cubic Clustering Criterion, pseudo $F$ and $t^{2}$ statistics revealed a two and a six cluster model. The first cluster $(n=23)$ was found in both models and consists of patients who present with an 'IL-5-high', 'IL-25-high', 'IL-17A-high', 'IL-17F-high', 'IL-10-high' profile. In the two-cluster model, the second patient cluster was defined as 'IL-17F-low'. In the sixcluster model, this group was divided into 5 separate patient clusters: 'IL-5- and IL10-high' but 'IL-17F-low' $\operatorname{profile}(n=7)$, 'IFN- $\gamma$-high' profile $(n=15)$, 'IL-6- and/or TNF-high' profile $(n=15)$, 'IL-22-high' profile $(n=15)$ and finally those with either an 'IL-4- and/or IL-13-high' profile or 'cytokine-low' profile $(n=130$; cluster 6). Patients in cluster 1 were characterised by worst lung function parameters $\left(\mathrm{FEV}_{1 \%}\right.$ predicted and $\mathrm{FEF}_{25-75 \%}$ ). Eosinophilic airway inflammation was highest in cluster 6 and in particular those patients with an 'IL-4- and/or IL-13-high' profile. High sputum neutrophils were seen cluster 1,2 and 4.

Conclusion: Each individual patient could be classified in one out of six unique clusters, defined by their sputum cytokine profile. Identifying specific patterns of cytokine expression in subtypes of asthma might be used in selecting those asthmatics that may benefit the most from treatment with monoclonal antibodies against specific cytokines.

\section{4 \\ Reduction of IgE by intensified anti- inflammatory treatment in patients with atopic asthma \\ Syk, $\mathrm{J}^{1}$; Malinovschi, $\mathrm{A}^{2}$; Borres, $\mathrm{M}^{3}$; Alving, $\mathrm{K}^{3}$ 'Department of Neurobiology, Karolinska Institutet, Care Sciensis and Society, Huddinge, Sweden; ${ }^{2}$ Department of Medical Sciences, Uppsala University, Uppsala, Sweden; ${ }^{3}$ Department of Women's and Children's Health, Uppsala University, Uppsala, Sweden}

Background: $\operatorname{IgE}$ mediates the development of airway inflammation in atopic asthma and inhibition of IgE with monoclonal antibodies is used to treat severe atopic asthma. However, the levels of total and specific $\operatorname{IgE}$ are considered to be rela- tively inert if allergen exposure is constant, and the effect of ordinary anti-inflammatory asthma treatment on IgE levels has been little studied. Both $\mathrm{IgE}$ production and increased exhaled NO (FeNO) levels are driven by the Th2 cytokines IL- 4 and IL-13, and FeNO has been shown to be reduced by inhaled corticosteroid (ICS) and leukotriene receptor antagonist (LTRA) treatment.

Method: We performed a post-hoc analysis of data from 158 patients with asthma and perennial allergy (age 18-64 years) from a randomised controlled study in primary health care (the NOAK study). The ICS and LTRA treatment in this study was guided by symptoms $(n=77)$ or FeNO $(n=81)$. Venous blood was sampled, FeNO was measured (NIOX MINO) and a quality of life questionnaire (Juniper miniAQLQ) was applied at baseline and 12 months. Total $\operatorname{IgE}$ and $\operatorname{IgE}$ antibodies against aero- and food allergens were analysed by ImmunoCAP.

Results: Total $\operatorname{IgE}$ was reduced by $10 \%$ $(P<0.001)$ over the 12 -months observation period. The sum of $\mathrm{IgE}$ antibody titres against 6 common perennial allergens was reduced by $16 \%(P<0.001), \operatorname{IgE}$ against 3 common seasonal allergens was reduced by $17 \% \quad(P<0.001)$ and $\mathrm{IgE}$ against food allergens (fx5) was reduced by $8 \%$ $(P=0.004)$. The changes were not related to any change in allergen exposure, and specific $\mathrm{IgG}_{4}$ levels remained unaltered. The decrease in IgE against perennial allergens related to both mean ICS dose $(P=0.030)$ and months on LTRA $(P=0.013)$ as well as with a reduction in FeNO $(P=0.003)$. The reduction in $\operatorname{IgE}$ against perennial allergens correlated with improvements in the mini-AQLQ score $(P=0.009)$.

Conclusion: Intensified anti-inflammatory treatment with ICS and LTRA in asthma patients with ongoing treatment resulted in reduced total and specific IgE levels which were unrelated to the degree of allergen exposure. Instead, the reduction in IgE was related to successful anti-inflammatory treatment leading to a reduction in FeNO. A decrease in IgE levels seemed important to gain improvement in asthma-related quality of life. The results suggest longterm beneficial effects of keeping FeNO as low as possible.
135

Identifying novel factors beyond prior hospitalisations, including biomarkers, associated with future asthma hospitalisation risk from routine UK primary care clinical records

Price, $\mathrm{DB}^{1,2}$; Bleecker, $\mathrm{ER}^{3}$; Campbell, $\mathrm{JD}^{4}$; Corrigan, $\overline{\mathrm{CJ}^{5} ; \text { Pavord, ID }}{ }^{6}$; Wenzel, $\mathrm{SE}^{7}$; Thomas, $\mathrm{M}^{8}$; Wilson, $\mathrm{AM}^{9}$; Clark, $\mathrm{A}^{9}$; Rigazio, $\mathrm{A}^{2}$; Burden, $\mathrm{A}^{2}$; Ashton, $\mathrm{VL}^{2}$; Gopalan, $\mathrm{GH}^{10}$; Buatti Small, $\mathrm{M}^{10}$

${ }^{1}$ Centre for Academic Primary Care, University of Aberdeen, Aberdeen, United Kingdom; ${ }^{2}$ Research in Real Life (RiRL) Ltd., Cambridge, United Kingdom; ${ }^{3}$ Wake Forest School of Medicine, Winston-Salem, NC, United States; ${ }^{4}$ University of Colorado, Aurora, IL, United States; ${ }^{5}$ King's College London, London, United Kingdom; ${ }^{6}$ University of Oxford, Oxford, United Kingdom; ${ }^{7}$ University of Pittsburgh, Pittsburgh, PA, United States; ${ }^{8}$ University of Southampton,

Southampton, United Kingdom; ${ }^{9}$ University of East Anglia, Norwich, United Kingdom; ${ }^{10} \mathrm{Teva}$ Pharmaceutical Industries Ltd, Frazer, PA, United States

Background: The aim of this study is to identify novel factors associated with future asthma hospitalisation risk in a real life setting from UK primary care clinical records.

Method: This is a historic, observational study using the Clinical Practice Research Datalink of asthma patients aged 1280 years with UK Hospital Episode Statistics data and no other chronic respiratory disease. The study period was 1 year before (baseline) and 1 year after (outcome) date of last blood eosinophil count.

Outcome: Asthma-related hospitalisation (inpatient admission or ER visit). Baseline characteristics: biomarkers; demographics; co-morbidities and markers of disease severity. Univariate logistic regression was used to identify characteristics associated with $\geq 1$ hospitalisation $(P \leq 0.05)$. The characteristics were then included in a multivariate model and reduced to a list of non-collinear factors.

Results: Of 61861 patients [34.4\% male; median (IQR) age $49(36,63)$ years] $1.7 \%$ (baseline) and 1.6\% (outcome) were hospitalised. The table shows factors associated with $\geq 1$ outcome hospitalisation (multivariate results).

Conclusion: Novel factors associated with future asthma hospitalisation risk identified through multivariate analysis include: elevated blood eosinophils; anaphylaxis; diabetes and a GP consultation for LRTI with prescription for antibiotics. Rhinitis is protective. 


\section{Oral Abstract Session}

\section{OAS 24 - Different facets in the management of food allergy}

136

Trial of incomplete avoidance for severe wheat or cow's milk allergy for their better prognosis

Okada, Y; Yanagida, N; Sato, S; Ogawa, A; Ogura, K; Nagakura, K; Emura, S; Asaumi, T; Ogura, K; likura, K; Ebisawa, M

${ }^{1}$ Department of Allergy, Clinical Research Center for Allergy and Rheumatology, Sagamihara National

Hospital, Sagamihara, Kanagawa, Japan

Introduction: Children with a history of symptoms after taking a slight amount of wheat (W) or cow's milk (CM) (immediate reaction group, IG), and children who have never taken $\mathrm{W}$ or $\mathrm{CM}$ due to high value of specific $\operatorname{IgE}(\mathrm{s} \operatorname{IgE}$ ) (no ingestion group, NG) are high possibility of positive oral food challenge (OFC). Therefore they have to eliminate $\mathrm{W}$ or $\mathrm{CM}$ completely. We analyzed whether OFC with $2 \mathrm{~g}$ of Udon noodle corresponding to $53 \mathrm{mg}$ of $\mathrm{W}$ protein (W0) or $3 \mathrm{ml}$ of heated $\mathrm{CM}$ (M0) to these two groups contributed daily intake of $\mathrm{W}$ or $\mathrm{CM}$ product.

Methods: W0 was performed in 80 children (74 of W-IG, 6 of W-NG) and M0 was per- formed in 161 children (141 of CM-IG, 20 of CM-NG) between Jul 2012 and Nov 2013. The median age of children was $3.6 \mathrm{y}$ for $\mathrm{W}$ and $4.8 \mathrm{y}$ for $\mathrm{CM}$, respectively.

Results: Prior to W0 or M0, the median previous threshold was $13.5 \mathrm{~g}$ of Udon noodle for $\mathrm{W}-\mathrm{IG}$ and $12.5 \mathrm{ml}$ of heated CM for CM-IG. The median W-sIgE (kU/ 1) was 27.4 for W-IG, 70.3 for W-NG and 29.0 for all $\mathrm{W}$-allergic children (W-ALL). The median CM-sIgE was 27.4 for CMIG, 62.2 for CM-NG and 31.1 for all CMallergic children (CM-ALL). The positive rate (PR) of W0 was $45.3 \%$ for $\mathrm{W}-\mathrm{IG}$, $33.0 \%$ for $\mathrm{W}-\mathrm{NG}$ and $45.0 \%$ for W-ALL. PR of M0 was $54.6 \%$ for CM-IG, $55.0 \%$ for CM-NG and $54.7 \%$ for CM-ALL. In children with $\operatorname{sIgE} \geqq 100$, PR of W0 was $78.6 \%(11 / 14)$, and PR of M0 was $78.3 \%$ (18/23). Children who passed W0 or M0 were allowed to take seasoning containing $\mathrm{W}$ or $\mathrm{CM}$ product within $3 \mathrm{ml}$ of milk such as butter. We conducted the retrospective review of children who had a history of $\mathrm{W}$ or $\mathrm{CM}$ allergy and passed $\mathrm{W} 0$ or M0 more than 1 year ago. The review revealed that 4 of $7 \mathrm{~W}$-allergic children could take $\mathrm{W}$ freely and 2 of $6 \mathrm{CM}$-allergic children could take $25 \mathrm{ml}$ or less of CM.

Conclusion: About one-half of the children completely eliminating $\mathrm{W}$ or $\mathrm{CM}$ passed W0 or M0, which resulted in taking a slight amount of $\mathrm{W}$ or $\mathrm{CM}$ product in daily life. We are going to compare the outcome between incomplete vs complete avoidance in the future.

\section{7 \\ A novel synbiotic concept derived from cow's milk-free source materials reduced allergic symptoms in preventive and therapeutic models for hen's egg allergy \\ van Esch, BCAM ${ }^{1,2}$; Diks, MAP ${ }^{1}$; Dingjan, $\mathrm{GA}^{1}$; \\ Harthoorn, $\mathrm{LF}^{3}$; Garssen, $\mathrm{J}^{1,2}$; Vos, $\mathrm{AP}^{2}$ \\ ${ }^{1}$ Utrecht Institute for Pharmaceutical Sciences, Utrecht University, Utrecht, The Netherlands; ${ }^{2}$ Nutricia \\ Research, Utrecht, The Netherlands; ${ }^{3}$ Nutricia Advanced Medical Nutrition, Nutricia Research, Utrecht, The} Netherlands

Background: For applications in severe cow's milk allergic (CMA) infants, a new

\begin{tabular}{|c|c|c|c|c|}
\hline Potential Risk Factor & Reference Category & Category & Odds Ratio (95\% Cl) & $P$-Value \\
\hline $\begin{array}{l}\text { Biomarker: blood eosinophil } \\
\text { count }\end{array}$ & $\leq 400 / \mu \mathrm{l}$ & $>400 / \mu l$ & $1.27(1.08,1.50)$ & 0.005 \\
\hline Prior hospitalisations & No hospitalisation & $\geq 1$ hospitalisation & $6.54(5.32,8.04)$ & $<0.001$ \\
\hline $\begin{array}{l}\text { Acute courses of oral } \\
\text { corticosteroids }\end{array}$ & No course & $\begin{array}{l}1 \text { course; } 2 \text { courses; } \geq 3 \\
\text { courses }\end{array}$ & $\begin{array}{l}1.74(1.44,2.09) ; 2.66(2.10 \\
3.36) ; 3.13(2.54,3.86)\end{array}$ & $\begin{array}{l}<0.001 \\
<0.001 \\
<0.001\end{array}$ \\
\hline Anaphylaxis at any time & No episode & $\geq 1$ episode & $2.20(1.29,3.76)$ & 0.004 \\
\hline GINA management step & $1-2$ & $0 ; 3 ; 4-5$ & $\begin{array}{l}1.77(1.39,2.24) ; 1.11(0.90 \\
1.37) ; 1.58(1.36,1.85)\end{array}$ & $\begin{array}{c}<0.001 \\
0.352 \\
<0.001\end{array}$ \\
\hline BMI & Normal (18.50-24.99kg/m²) & $\begin{array}{l}\text { Underweight }\left(<18.50 \mathrm{~kg} / \mathrm{m}^{2}\right) \\
\text { Overweight }\left(25-29.99 \mathrm{~kg} / \mathrm{m}^{2}\right) \\
\text { Obese }\left(\geq 30 \mathrm{~kg} / \mathrm{m}^{2}\right)\end{array}$ & $\begin{array}{l}1.63(1.19,2.23) ; 0.80(0.67 \\
0.96) ; 0.89(0.76,1.05)\end{array}$ & $\begin{array}{l}0.002 ; 0.018 \\
0.170\end{array}$ \\
\hline Diabetes (type 1 or 2) & No diagnosis and/or therapy & Diagnosis and/or therapy & $1.46(1.27,1.68)$ & $<0.001$ \\
\hline $\begin{array}{l}\text { GP consultations for LRTIs with } \\
\text { prescriptions for antibiotics }\end{array}$ & $\begin{array}{l}\text { No consultation and } \\
\text { prescription }\end{array}$ & $\begin{array}{l}1 \text { consultation and } \\
\text { prescription; } \geq 2 \text { consultations } \\
\text { and prescriptions }\end{array}$ & $1.37(1.16,1.62) ; 1.22(0.96,1.55)$ & $<0.001 ; 0.098$ \\
\hline $\begin{array}{l}\text { Smoking status * Ischaemic } \\
\text { heart disease * Paracetamol } \\
\text { (acetaminophen) * Anxiety } \\
\text { and/or depression * Age * } \\
\text { Rhinitis }\end{array}$ & $\begin{array}{l}\text { Non-smoker } * \text { No diagnosis } \\
* \text { No prescription * No } \\
\text { diagnosis *12-60 years * } \\
\text { No diagnosis and/or } \\
\text { therapy }\end{array}$ & $\begin{array}{l}\text { Current smoker; Former } \\
\text { smoker } * \text { Diagnosis * } \geq 1 \\
\text { prescription * Diagnosis *61- } \\
80 \text { years * Diagnosis and/or } \\
\text { therapy }\end{array}$ & $\begin{array}{c}1.28(1.08,1.52) ; 1.10(0.94,1.28) \\
* 1.28(1.02,1.60) * 1.23(1.07 \\
1.41) * 1.21(1.06,1.38) * 1.21 \\
(1.04 .1 .40) * 0.81(0.71,0.92)\end{array}$ & $\begin{array}{l}0.006 ; 0.239 \\
* 0.035 * \\
0.004 * \\
0.005 * \\
0.012 * \\
0.002\end{array}$ \\
\hline
\end{tabular}

Factors associated with $\geq 1$ hospitalisation.

* Denotes row division. 
synbiotic concept was designed from source materials and probiotic bacteria that were cultured completely cow's milkfree. The preventive and therapeutic effects of this concept were tested in models for hen's egg allergy.

Methods: Mice were orally sensitised once a week for 5 weeks to ovalbumin (OVA) using cholera toxin as an adjuvant. Non-sensitised mice received cholera toxin in PBS only.

Mice were either fed the synbiotic concept (short-chain fructo-, long-chain fructo- and acidic oligosaccharides plus Bifidobacterium breve M-16V) continuously from day -14 or for 3 weeks starting after the last sensitisation. Anaphylactic shock reactions, a drop in body temperature and acute allergic skin responses were measured as clinically-related allergic symptoms upon challenge to OVA. Allergen-specific IgE and $\mathrm{mMCP}-1$, as a reflection of intestinal mast cell degranulation were measured in serum. TGF-beta and IL-10 were determined in ileum using PCR.

Results: Anaphylactic shock symptoms, a drop in body temperature, an acute allergic skin response, enhanced levels of mMCP-1 and specific OVA-IgE were observed in sensitised mice. Interestingly, the synbiotic concept reduced anaphylactic shock symptoms, the changes in body temperature and the acute allergic skin response in both the preventive and therapeutic settings. In the therapeutic setting mMCP-1 was also reduced. mRNA expression of TGF-beta, but not of IL-10, was enhanced in the preventive setting.

Conclusion: A new synbiotic concept, suitable for the dietary management of severe CMA, shows relevant immunomodulatory capacity if fed to both naïve and already sensitised mice, suggesting potential benefits on allergy-related parameters in humans. Although an increase in local TGF-beta expression was observed, further research is needed to elucidate the mechanisms underlying the observed effects.

\section{8 \\ MIRABEL: a research program to develop tools for risk and cost/benefit analysis of peanut allergy \\ Crépet, $A^{1}$; Papadopoulos, $A^{1}$; Elégbédé, $C F^{1}$; Ait-Dahmane, $\mathrm{S}^{1}$; Deschildre, $\mathrm{A}^{2}$; Just, $\mathrm{J}^{3}$; Bruyère, $\mathrm{O}^{4}$; Roosen, $\mathrm{J}^{5}$; Marette, $\mathrm{S}^{6}$; Moneret-Vautrin, D-A ${ }^{7}$ ${ }^{1}$ ANSES, French Agency for Health and Safety, Maisons-Alfort, France; ${ }^{2}$ Jeanne de Flandre Hospital, Lille CHU, Lille, France; ${ }^{3}$ Trousseau Hospital, Paris, France; ${ }^{4}$ Liège University, Liège, Belgium; ${ }^{5}$ Technical University of Munich, Munich, Germany; ${ }^{6}$ INRA, National Institute of Agronomic Research, Thiverval- Grignon, France; ${ }^{7}$ Allergovigilance Network, \\ Vandoeuvre Les Nancy, France}

Background: Traces of allergenic food represent a significant health risk for allergy sufferers. Knowledge is missing on the different risk components and particularly on the link between presence of traces of allergens in food, food consumption behaviours of allergy sufferers and thresholds of reaction. There is also a lack of risk-based guidance and operational tools for the different stakeholders.

Method: Each risk component was investigated to accurately characterise the risk of peanut allergy and to test risk management options. A survey to peanut allergy sufferers was conducted in France and Belgium. Patients were recruited by 71 allergists during their consultation. Data on clinical characteristics of allergic patients, thresholds of reactions, consumptions behaviours related to peanut containing products were collected by a medical and a consumption questionnaire. For the most consumed products by the allergic population, peanut traces were quantified. A risk model based on a Bayesian Network was developed and applied to the collected data. A cost-benefit analysis was conducted to anticipate impacts of new labels.

Results: During 21 months, 792 medical and around 400 consumption questionnaires were returned fully completed. $85 \%$ of patients were children. The modelling of the 264 thresholds from oral challenges shows that $1 \%$ of the population react to a dose lower than $0.5 \mathrm{mg}$ of peanut protein. $1 \%$ of the most consumed food by allergy sufferers contains peanut protein with a maximum of $0.45 \mathrm{mg} / 100 \mathrm{~g}$. A high preference for the label 'guaranted without', especially since parents are anxious, was observed. Consumption behaviours are influenced by the fact that they also have a tree nuts allergy, severe symptoms and they know their allergy since more than one year.

Conclusion: This program provides a full of relevant and original data integrated in an operational framework for allergic risk analysis, in order to improve quality of life of allergy sufferers.

139

Severe anaphylaxis in chidren with $\lg \mathrm{E}$ mediated food allergy, caused by oral immunotherapy

Pajno, GB; Caminiti, L; Crisafulli, G; Pajno, C; Chiera, F $\overline{\text { University }}$ of Messina, Messina, Italy

Background: The safety represents a paramount issue for the active treatment of $\operatorname{IgE}$ mediated food allergy with Oral Immunotherapy(OIT).The aim of this survey was the detection of the rate of Anaphylaxis during immunotherapy with cow's milk (CM-OIT) or hen's egg(HE- OIT).
Method: Since 2006 we have treated $n .80$ subjects, aged 4 to15 years(median 9 years.), suffering from IgE mediated food allergy; $n .48$ with CM- OIT and $n .32$ with HE-OIT. We used the weekly up-dosing protocol. Reactions' severity was assessed through the 'Grading of Food Induced Anaphylaxis according to Severity of Clinical Symptoms' which could be summarized from grade 1 to 5 .Grade(s) from 3 to 5 were considered as severe of life threatening events.

Results: During CM- OIT two patients had symptoms of grade 3 and two patients had symptoms of grade $4(8 \%)$, none had symptoms of grade 5.During HE-OIT two patients had symptoms of grade 3 and one of grade $4(9 \%)$, none had symptoms of grade 5.These subjects had successfully treated with i.m. adrenaline, plus other rescue medications and desensitisation protocol was stopped.Of note, the appearance of mild to moderate adverse effects was quite frequent in remaining patients.

Conclusion: Among subjects treated with OIT: a minority of them had severe anphylactic reactions which were appropriately controlled with i. m. adrenaline.Therefore, OIT could be considered rather safe in the majority of subjects treated with either CM- OIT or HE- OIT.However, adverse events during oral immunotherapy are largely impredictable, so far it could be performed in selected medical centres and under strict medical supervision because of safety concerns.

\section{0 \\ Assessing the impact of adrenaline autoinjector prescription and ownership for families of children with food allergies \\ Allen, $\mathrm{WC}^{1,2,3}$; Bidarkar, $\mathrm{M}^{1}$; Van Nunen, $\mathrm{S}^{2,3}$; Campbell, $\mathrm{DE}^{3,4}$ \\ ${ }^{1}$ General Medicine, Children's Hospital at Westmead, Westmead, NSW, Australia; ${ }^{2}$ Allergy, Royal North Shore Hospital, Sydney, NSW, Australia; ${ }^{3}$ University of Sydney, Sydney, NSW, Australia; ${ }^{4}$ Allergy and Immunology, Childrens Hospital at Westmead, Sydney, NSW, Australia}

Background: Adrenaline is frequently prescribed for children at risk of anaphylaxis, however it is not clear whether prescription of adrenaline autoinjectors (AA) influences parental burden and stress.

Method: Questionnaires were sent to parents of all food allergic children (FAC) attending allergy clinics for review at two Sydney hospitals in May-September 2013. The questionnaire ascertained parental attitudes, confidence and knowledge regarding AA's and included the validated, Food Allergy Quality of Life-Parental Burden (PB) questionnaire (Cohen et al, 2004). 
Mean Parental Burden score was calculated.

Results: The response rate was $68 \%(165 /$ $244)$. Of FAC, $62 \%$ were male aged 117 years (median 6.0). The proportion of children with multiple food allergies was high, with allergy to eggs, peanut, treenuts and cow milk most common. A past history of anaphylaxis was reported in $63 \%$ and $42 \%$ reported reactions in the last 6 months. Rates of coexistant eczema $(85.4 \%)$ and asthma $(54.0 \%)$ were high.

AA had been prescribed for $84 \%$ of FAC. Of these, $12 \%$ had previously had an AA administered. Of those $(n=27)$ not prescribed an AA, $77 \%$ of parents believe that an AA was not necessary for management. 26\% would feel more comfortable with an AA.

FAC with a past history of anaphylaxis were significantly more likely to have had an AA prescribed $(P<0.0001)$. There was no significant difference in FAQL-PB mean scores with age or AA prescription status. The PB score was significantly greater if the parent had administered the AA in the past $(P=0.02)$ and where there was a history of anaphylaxis $(P=0.048)$.

I3\% of parents whose FAC children were prescribed an AA had an increase in stress related to allergy management. $90 \%$ of parents reported that the AA increased or did not change the child's quality of life, the family or FACs freedom.

Summary: This study provides supportive data that severity of food allergy and past anaphylaxis rather than prescription of an AA appear to be major influences on parental burden.

\section{1}

A phase II randomised controlled trial of peanut oral immunotherapy in children

Anagnostou, $A^{1}$; Islam, $S^{1}$; King, $Y^{2}$; Foley, $L^{2}$; Pasea, $\mathrm{L}^{3}$; Bond, $\mathrm{S}^{4}$; Deighton, $\mathrm{J}^{2}$; Ewan, $\mathrm{P}^{1,2}$; Clark, $\mathrm{A}^{1,2}$ ${ }^{1}$ University of Cambridge, Department of Medicine, Cambridge, United Kingdom; ${ }^{2}$ Cambridge University Hospitals, Department of Allergy, Cambridge, United Kingdom; ${ }^{3}$ Department of Public Health and Primary Care, University of Cambridge, Centre for Applied Medical Statistics, Cambridge, United Kingdom; ${ }^{4}$ Addenbrooke's Hospital, Cambridge Clinical Trials Unit, Cambridge, United Kingdom

Background: Small studies suggest peanut oral immunotherapy (OIT) may be effective in peanut allergy.

Method: In this phase-II randomised controlled crossover trial we assigned 99 participants (7-16 years) with peanut allergy to active oral immunotherapy (peanut flour, 2-800 mg) or control (peanut avoidance-current standard of care). Primary outcome was desensitisation: negative peanut challenge at 6 months (phase 1). Control participants also underwent OIT (phase 2), followed by DBPCFC. Immunological parameters and quality of life were measured.

Results: The primary outcome, desensitisation, was observed in $62 \%$ of the active group and none of the control group after phase $1[95 \%$ confidence intervals (CI) $45-78 \%$ v $0-9 \% ; P<0.001] ; 84 \%$ (CI 70$93 \%$ ) of the active group maintained ingestion of $800 \mathrm{mg}$ protein (five peanuts) per day. Median increase in peanut threshold after OIT was $1345 \mathrm{mg}$ (range 45$1400 \mathrm{mg} ; \quad P<0.001$ ): a 25.5 -fold (range 1.82-280; $\quad P<0.001)$ median increase. After phase 2, 91\% (CI 79-98\%) maintained ingestion of $800 \mathrm{mg}$ protein (five peanuts) per day and 54\% (CI 35-72\%) tolerated a $1400 \mathrm{mg}$ (10 peanuts) challenge. Quality of life scores improved (decreased) after OIT [median -1.61 (range -4.87 to $0.24 ; P<0.001)]$. Side effects included gastrointestinal symptoms after $2.2-6.3 \%$ of doses and wheeze after $0.41 \%$ of doses (1/ 5 th participants). Intramuscular adrenaline was used after $0.01 \%$ of doses (1 participant).

Conclusion: Peanut OIT offers a highly effective treatment for peanut allergy.In children with peanut allergy of any severity, OIT successfully induced desensitisation in the majority, with a clinically meaningful increase in peanut threshold. A high response rate also occurred in the control group after OIT. Immunological changes reflected clinical desensitisation. Quality of life improved after intervention and the safety profile was good. These findings require further study in a wider population; peanut OIT should not be undertaken in non-specialist settings. 


\section{Oral Abstract Session}

\section{OAS 25 - Diagnostic tools in allergic and non-allergic rhinitis}

\section{2 \\ Lower threshold for TRPV1 and TRPA1 activation of sensory nerves in idiopathic rhinitis patients}

Van Gerven, $\mathrm{LH}^{1,2}$; Alpizar, YA ${ }^{3}$; Dekimpe, $\mathrm{E}^{1}$; Steelant, $\overline{\mathrm{B}^{2} ; \text { Talavera, } \mathrm{K}^{3}}$; Hellings, $\mathrm{PW}^{1,2}$; Clinical Immunology ${ }^{1}$ Otorhinolaryngology, Head \& Neck Surgery, University Hospitals Leuven, Leuven, Belgium; ${ }^{2}$ Laboratory of Clinical Immunology, Department of Microbiology and Immunology, KULeuven, Leuven, Belgium; ${ }^{3}$ Laboratory for Ion Channel Research, Department of Molecular Cell Biology, KULeuven, Leuven, Belgium

Background: We recently showed that the therapeutic action of capsaicin treatment in idiopathic rhinitis (IR) is based on the ablation of the TRPV1-Substance P nociceptive signaling pathway (Van Gerven et al., JACI 2013). However, the functional consequences of capsaicin treatment on nasal nerve activation remain unknown.

Method: A double-blinded placebo-controlled trial was designed, involving 25 IR patients and $14 \mathrm{HC}$ so far. Nasal mucosal potentials (NMP) were evoked by exposing the nasal mucosa of IR patients and HC to an aerosol with different TRP-agonists. Low concentrations of 3 irritants (cinnamaldehyde, allyl isothiocyanate $(=$ mustard oil, MO) and capsaicin) that activate TRPA1 and/or TRPV1 were applied. The threshold for each irritant was determined for each individual as the dose at which a clear change from baseline in NMP was recorded.

Results: Baseline TRPV1 expression was significantly higher in IR patients compared to HC $(P<0.001)$. The threshold for $\mathrm{MO}$ was $5 \mathrm{mM}$ in $9 \%, 10 \mathrm{mM}$ in $18 \%$ and $20 \mathrm{mM}$ in $73 \%$ of $\mathrm{HC}$, whereas for IR patients, $31.5 \%$ responded by an NMPdecrease at $5 \mathrm{mM}, 37 \%$ on $10 \mathrm{mM}$ and $31.5 \%$ on $20 \mathrm{mM}$. The MO-threshold was overall significantly lower for IR patients compared to $\mathrm{HC}(P=0.0482)$. Remarkably, no changes in NMP measurements could be documented in IR patients and HC after cinnamaldehyde exposure, and capsaicin exposure induced cough that hampered NMP recording.

Conclusion: The lower chemical threshold to react to MO and the over-expression of TRPV1 in IR patients compared to $\mathrm{HC}$ confirmed the specific role of these nociceptor in the pathophysiology of IR, which in turn, may help to design more specific and effective therapies. The time-frame will give us 'functional information' on the Cfiber innervation before the treatment, during the phase of therapeutic response and during the period of recurrence of the IR symptoms.

\section{3 \\ Use of paper filter discs for measurement of local biomarkers of nasal mucosal inflammation}

Berings, $\mathrm{M}^{1}$; Calus, $\mathrm{L}^{1}$; Devuyst, $\mathrm{L}^{1}$; Thibaut, $\mathrm{VZ}^{1}$; De Ruyck, $\mathrm{N}^{1}$; Bachert, $\mathrm{C}^{1,2}$; Gevaert, $\mathrm{P}^{1}$

${ }^{1}$ Upper Airway Research Laboratory, Ghent University, Ghent, Belgium; ${ }^{2}$ Division of ENT Diseases, Karolinska Institutet, Clintec, Stockholm, Sweden

Background: Allergic rhinitis has been characterised by an important local mucosal inflammation. Therefore we aimed to measure mucosal immunoglobulins and tryptase in nasal secretions by means of paper filter discs in allergic rhinitis (AR) patients in comparison to controls. The purpose of this study was to evaluate the suitability of this approach for use in future clinical trials.

Method: Nasal secretions from 12 patients with AR to grass pollen and 12 healthy controls were collected. Two pre-weighed filter discs were placed bilaterally under direct visualization on the anterior third of the nasal septum. Five minutes later, they were removed and weighted again. After adding $2 \mathrm{ml} 0.9 \% \mathrm{NaCl}$ solution to the discs and centrifugation, the nasal secretions were stored in aliquots at $-20^{\circ} \mathrm{C}$ until analysis. Biomarkers such as total $\mathrm{IgE}$, grass pollen specific $(\mathrm{gx} 3) \mathrm{IgE}$, total $\mathrm{IgG}, \mathrm{IgG} 4$ and tryptase were measured. Patients with AR were selected based on symptoms during the grass pollen season and based on a positive skin prick test for grass pollen. Control patients were healthy patients without rhinological or allergic disease and with negative skin prick test to the standard panel of allergens.

Results: In nasal secretions of AR patients, significant higher levels of total $\operatorname{IgE}[43.13 \mathrm{kU} / 1(2.83-75.36)$ vs $1.66 \mathrm{kU} / 1$ (1.66-3.21) resp., $P=0.006]$, grass pollen specific $\operatorname{IgE}[14.11 \mathrm{kU} / 1 \quad(2.30-24.56)$ vs $1.66 \mathrm{kU} / 1(1.66-1.66)$ resp., $P=0.001]$ and tryptase $\quad[33.67 \mu \mathrm{g} / 1 \quad(8.28-85.07) \quad$ vs $8.28 \mu \mathrm{g} / 1 \quad(8.28-8.28), \quad P=0.032] \quad$ were found compared to controls. Total $\mathrm{IgG}$ levels tend to be lower in AR patients compared to controls [182.62 mg/l (86.04$314.56) \quad$ vs $\quad 256.17 \mathrm{mg} / \mathrm{l} \quad(192.67-457.25)$, $P=0.288]$ and the levels of IgG4 were rather similar in both groups $[12.87 \mathrm{mg} / 1$ (4.79-53.48) vs $16.75 \mathrm{mg} / \mathrm{dl} \quad(3.36-33.26)$, $P=0.833]$.

Conclusion: We were able to measure different biomarkers of nasal mucosal inflammation in nasal secretions collected by means of paper filter discs. As this approach is considerably less invasive and requires less expertise than nasal biopsy, and as -in this small sample- patients with AR had significant higher levels of total IgE, specific IgE and tryptase compared to healthy controls, this method seems to be suitable for monitoring local mucosal inflammation in allergic rhinitis.

\section{4 \\ Local allergic rhinitis and non-allergic rhinitis: different demographic and clinical phenotypes}

Rondón, $\mathrm{C}^{1}$; Campo, $\mathrm{P}^{1}$; Blanca-López, $\mathrm{N}^{2}$; Salas, $\mathrm{M}^{1}$; Cañamero, $\mathrm{MD}^{1}$; Sanchez, $\mathrm{MI}^{1}$; Posadas, $\mathrm{T}^{1}$; Bogas $\mathrm{G}^{1}$; Torres, $\mathrm{MJ}^{1}$; Canto, $\mathrm{G}^{2}$; Blanca, $\mathrm{M}^{1}$ ${ }^{1}$ Allergy Unit, IBIMA, Regional University Hospital of Malaga, UMA, Malaga, Spain; ${ }^{2}$ Allergy Unit, Infanta Leonor Hospital, Madrid, Spain

Background: The development of local allergic rhinitis (LAR) in subjects without systemic atopy, highlights the need for a clinical phenotyping of LAR and NAR patients. In this study we analyzed the demographic-clinical phenotyping of subjects with NAR and LAR, and investigated the presence of risk factors in LAR.

Method: A total of 280 patients $(\mathrm{NAR}=140$ and $\mathrm{LAR}=140)$ were studied. A clinical history and demographicclinical questionnaire were performed. The local ethics committee approved the study, and all participants gave informed consent. Results: The study of the demographic characteristics showed NAR subjects were significantly older than LAR (NAR:42.15 $\pm 16.75 ; \quad$ LAR:29.51 \pm 12.47 ; $P=0.002$ ), and had lower association with female gender $(\mathrm{OR}=0.50 ; 95 \% \mathrm{CI}, 0.28$ $0.88 ; P=0.015)$, family history of atopy (FHA) $\quad(\mathrm{OR}=0.20 ; 95 \%$ CI, 0.09-0.45; $P=0.001)$, childhood onset $(\mathrm{OR}=0.19$; 95\% CI, 0.10-0.38; $P=0.001$ ), and urban 
household $(\mathrm{OR}=0.44 ; 95 \% \mathrm{CI}, 0.23-0.82$; $P=0.009)$. The clinical evaluation showed NAR patients had higher association with perennial rhinitis $(\mathrm{OR}=2.39 ; 95 \% \quad \mathrm{CI}$, 1.12-5.01, $\quad P=0.018)$ and urticaria $(\mathrm{OR}=4.42 ; \quad 95 \% \quad \mathrm{CI}, \quad 1.37-14.29$; $P=0.008)$, and lower association to severe symptoms $(\mathrm{OR}=0.18 ; 95 \% \mathrm{CI}, 0.09-0.36$; $P=0.001)$, conjunctivitis $(\mathrm{OR}=0.51 ; 95 \%$ CI, $0.29-0.91 ; \quad P=0.022)$ and asthma $(\mathrm{OR}=0.40 ; 95 \% \mathrm{CI}, 0.20-0.80 ; P=0.008)$ than LAR. Sneezing $(78.9 \%)$ in NAR and itching $(85.5 \%)$ in LAR were the main symptoms. The logistic regression identified female gender, FHA, childhood onset, asthma, seasonal rhinitis and severe symptoms as risk factors for the diagnosis of LAR; and mucous rhinorrea, urticaria, and symptoms triggered by chemical irritant as risk factor for NAR.

Conclusion: The detection of different demographic-clinical phenotypes and risk factors in LAR and NAR patients can be a useful tool for the identification of potential allergic rhinitis patients in subjects without systemic atopy.

Funding: National Health Ministry FIS PI11/02619.

\section{5}

The comparison of serum leptin, adinopectin and eosinophil cationic protein levels in patients with local allergic rhinitis

Atayık, $\mathrm{E}^{1} ;$ Abadoglu, $\mathrm{O}^{2}$

Clinical Immunology and Allergy, Diyarbakır Training and Education Hospital, Diyarbakır, Turkey; ${ }^{2}$ Clınıcal Immunology and Allergy, Cumhuriyet University Medical Faculty, Sivas, Turkey

Background: The aims of this study were to measure serum adinopectin, leptin and eosinophil cationic protein (ECP) levels in patients with local allergic rhinitis (LAR), who had perennial rhinitis symptoms, negative skin prick and/or intradermal (ID) tests, and positive or negative nasal provocation test (NPT) and to investigate whether there was a difference between patients with allergic rhinitis (AR) and LAR.

Method: Study included 30 patients with AR with sensitisation to Der $p$ and/or Der $f$ shown through skin tests, 30 patients with non-allergic rhinitis (NAR) whose skin tests showed no sensitisation to house dust mite (HDM), and 30 healthy controls. The subjects in NAR group and the control group underwent NPT with HDM antigen and the symptom scores were recorded. Serum leptin, adinopectin and ECP levels were measured in all subjects.

Results: NPT yielded positive results in $30 \%$ of patients with NAR but none in the control group. Serum ECP level was significantly higher in AR group compared to other groups and also significantly increased in LAR patients with positive NPT compared to LAR patients with negative NPT $(P=0.001)$. Serum leptin levels were similar between three groups. Within the NAR group, serum leptin level was significantly higher in LAR patients with positive NPT compared to those with negative NPT $(P=0.04)$. A relation between the severity of rhinitis and serum leptin and adiponectin levels was not identified neither in AR nor in LAR group. Serum adiponectin level was significantly higher in AR patients compared to other groups $(P=0.002)$.

Conclusion: Routine use of NPT may be suggested in patients with NAR as it may be instructive for long term management including protective measures and immunotherapy. Serum leptin and adinopectin levels were not efficient to discriminate between AR and LAR. Serum leptin level may be used to discriminate between NAR and LAR; a previous study on this subject could not be encountered in the current literature.

\section{6 \\ Use of basophil activation test for identifying subjects with local allergic rhinitis caused by $\boldsymbol{D}$. pteronyssinus \\ Campo, $\mathrm{P}^{1}$; Rondon, $\mathrm{C}^{1}$; Barrionuevo, $\mathrm{E}^{1}$; Gomez, $\mathrm{E}^{2}$; Bogas, ${ }^{1}$; Galindo, $\mathrm{L}^{1}$; Huertas, $\mathrm{JA}^{1}$; Melendez, $\mathrm{L}^{2}$; Mayorga, $\mathrm{C}^{2}$; Blanca, $\mathrm{M}^{1}$ \\ ${ }^{1}$ Allergy Unit, IBIMA, Regional University Hospital of Malaga, UMA, Malaga, Spain; ${ }^{2}$ Research Laboratory, IBIMA, Regional University Hospital of Malaga, UMA, Malaga, Spain}

Background: Previous studies showed the presence of allergen-specific IgE in the surface of peripheral basophils in $50 \%$ of subjects with local allergic rhinitis (LAR) due to D. pteronyssinus, although this phenomenon was investigated in a small number of patients. The aim of this study is confirming the presence of specific allergen activation of basophils, by means of basophil activation test (BAT) in a large population of LAR subjects sensitised to D. pteronyssinus (DP).

Method: BAT with 5, 20 and $50 \mathrm{ng} / \mathrm{ml}$ of DP extract was performed in 86 subjects: 30 with LAR (positive NPT with DP, negative skin testing and sIgE to DP), 32 with AR (positive NPT with DP, positive skin testing and $\operatorname{sIgE}$ to DP), 10 with NAR (negative NPT, skin testing and sIgE to DP, alternaria, phleum and olive tree pollen) and 14 healthy controls (negative NPT, negative skin testing and sIgE to DP). Local ethics' committee approved the study.
Results: BAT with DP was positive in 28 / 32 of AR patients (88) and in 14/30 (47\%) of LAR subjects. In healthy controls, $1 / 14$ had a positive response $(7 \%)$ and $1 / 10$ NAR subjects had positive results $(10 \%)$. BAT sensitivity was $85 \%$ in AR patients and $50 \%$ in LAR with a specificity of $93 \%$ for both groups.

Conclusion: Basophil activation test may be a useful tool to support the diagnosis of local allergic rhinitis.

\section{7 \\ Differentiating groups of rhinitis patients based on molecular markers in nasal secretions}

Segboer, CL; Holland, CT; Reinartz, SM; van Egmond, D; Terreehorst, I; Fokkens, WJ; van Drunen, CM Otolaryngology, Academic Medical Center, Amsterdam, The Netherlands

Background: In contrast to our detailed understanding of the molecular processes in allergic rhinitis (AR) we know relative little about non-allergic rhinitis (NAR) or about mixed rhinitis patients that suffer from AR ánd NAR. NAR patients only share a negative skin prick test or RAST for allergy and many different conditions (medication use, cold, smoke, stress, etc.) may trigger symptoms.

Method: A cross-sectional study was performed on and 23 NAR, 22 AR, 21 mixed rhinitis patients, and 23 healthy controls. Nasal secretions were obtained outside the pollen season and all patients had negative SPTs for perennial allergens. All rhinitis patients had moderate-severe rhinitis symptoms for at least one year with AR patients having a positive SPT for pollen. Idiopathic rhinitis patients had a negative SPT and no other identifiable causes of rhinitis symptoms. Mixed rhinitis patients had rhinitis symptoms for most of the year and only a positive SPT for pollen allergens. A healthy control group had no symptoms of rhinitis and a negative SPT. Nasal secretions were collected from the inferior meatus and levels for 30 mediators was determined using multiplex ELISA.

Results: Significant differences between groups were detected for IL-12 $(P=0.001)$ and $\operatorname{HGF}(P=0.02)$, with a trend for VEGF $(P=0.065)$ and INF- $\alpha(0.108)$. For these mediators the mixed rhinitis group showed statistically significant lower levels than healthy controls. In AR, IL-12, HGF, and INF- $\alpha$ median levels were also reduced to the level of mixed rhinitis but still had expression in some individuals.

Conclusion: The lower levels IL-12 and HGF in mixed rhinitis patients compared to AR suggest an interaction between the NAR phenotype and AR in these patients despite normal expression of these mediators in NAR. 


\section{Oral Abstract Session}

\section{OAS 26 - Autoimmunity}

148

No evidence of autoreactivity in nasal polyposis

De Schryver, $\mathrm{E}^{1}$; Calus, $\mathrm{L}^{1}$; Bonte, $\mathrm{H}^{1}$; Donovan, $\mathrm{E}^{2}$; Elewaut, $\mathrm{D}^{2}$; Valenta, $\mathrm{R}^{3}$; Mitterman, $\mathrm{I}^{3}$; Van Zele, $\mathrm{T}^{1}$; Gevaert, $\mathrm{P}^{1}$

${ }^{1}$ Otorhinolaryngology, Ghent University Hospital, Ghent, Belgium; ${ }^{2}$ Reumatology, Ghent University Hospital, Ghent, Belgium; ${ }^{3}$ Allergology, Medical

University of Vienna, Vienna, Austria

Background: Chronic rhinosinusitis with nasal polyps is an invalidating disease of the sinonasal mucosa. Still no effective medical treatment is available and recurrence after surgical removal of nasal polyps often occurs. Inflammation in nasal polyps is characterised by edema, local hyperglobulinemia, high local $\operatorname{IgE}$ concentrations and the presence of mucosal lymphoid accumulations.

The hypothesis that autoimmunity is a contributing factor in pathogenesis is based on the chronic heavily inflamed environment and because nasal polyps typically recur after surgical resection. This recurrence might be a result of self or autoimmunity.

Method: We performed an autologous serum skin test (ASST) in 32 subjects to identify anti-IgE or anti-FceRI autoantibodies. We included 15 controls and 17 CRSwNP patients.

In tissue homogenates and serum of 34 CRSwNP and 9 controls we tested for autoreactivity by an immunoprecipitation assay to detect anti-IgE autoantibodies and by line immunoassay (LIA). In the latter we tested for the presence of autoantibodies against RNP70k, RNPA, RNPC, SmD, SSA/Ro52，SSA/Ro60，SSB/La， CenpB, Topo1_Scl7, ribosomalP, histones and SmB.

Results: After intradermal injection of $0.05 \mathrm{ml}$ of undiluted autologous serum in ASST, no serum-induced wheal was $1.5 \mathrm{~mm}$ larger compared to control at $30 \mathrm{~min}$, thus no in vivo autoreactivity could be demonstrated.

The immunoprecipitation assay did not show any IgE autoreactivity in nasal tissue samples and serum to nitrocellulose-blotted protein extracts of a human epithelial cell line (A-431).

The LIA was performed by adding tissue homogenates, sera and nasal secretions to strips coated with antigens (RNP70k,
RNPA，RNPC，SmD，SSA/Ro52，SSA/ Ro60, SSB/La, CenpB, Topo1_Scl7, ribosomalP, histones and $\mathrm{SmB}$ ), but no autoimmune factors could be detected.

Conclusion: Based on our results from the ASST test, immunoprecipitation assay and LIA, there is no evidence for involvement of autoreactivity in pathogenesis of nasal polyposis.

\section{9 \\ Th17 and Treg expression in Kawasaki disease \\ Guo, $\mathrm{M}^{1}$; Tseng, W-N ${ }^{1}$; Ko, C- $\mathrm{H}^{1}$; Chang, L-S ${ }^{1}$; Wang, $\mathrm{L}^{1}$; Want, C- $\mathrm{L}^{2}$; Yang, $\mathrm{KD}^{3}$; Kuo, $\mathrm{H}-\mathrm{C}^{1,4}$ \\ ${ }^{1}$ Pediatrics, Kaohsiung Chang Gung Memorial Hospital, Kaohsiung, Taiwan; ${ }^{2}$ Pediatrics, Po-Jen Hospital, Kaohsiung, Taiwan; ${ }^{3}$ Chang Bing Show Chwan Memorial Hospital, Changhua County, Taiwan; ${ }^{4}$ Kawasaki Disease Center, Kaohsiung Chang Gung Memorial Hospital, Kaohsiung, Taiwan}

Background: Kawasaki disease is a vasculitis afflicting children of less than 5 years of age. Many autoimmune diseases including systemic lupus erythematosis, rheumatoid arthritis and multiple sclerosis have been found to involve an up-regulation of Th17 cells which promote autoimmunity, and a down-regulation of anti-inflammatory Treg cells. However, few studies have examined the Th17/Treg expression in Kawasaki disease.

Method: Blood samples were obtained from 92 children with Kawasaki disease at three time points: before IVIG therapy (KD1), 3 days (KD2) and 21 days (KD3) after completing IVIG therapy. Blood samples from 25 children with a febrile illness were also taken and used as a control. Plasma levels of Th17 and Treg related cytokines including IL-6, IL-17, IL-10 and TGF- $\beta$ were tested using Luminex ${ }^{100}$ beads system and ELISA. The expression levels of ROR $\gamma \mathrm{t}$ and Foxp3 were detected using real-time RT-PCR.

Results: Patients with Kawasaki disease prior to IVIG treatment had higher levels of plasma IL-17 $(22.72 \pm 3.36 \quad \mathrm{Vs}$ $7.23 \pm 1.9 \mathrm{pg} / \mathrm{ml}, \quad P<0.001), \quad$ IL-6 $(154.84 \pm 25.15$ vs $40.78 \pm 14.86 \mathrm{pg} / \mathrm{ml}$, $P<0.001)$ and TGF- $\beta(13278.95 \pm 1010.57$ vs $\quad 10226.37 \pm 1046.29 \mathrm{pg} / \mathrm{ml}, \quad P=0.039$ ) when compared to the febrile control. In children with Kawasaki disease, Th17 related cytokines IL-6 (KD1 $<\mathrm{KD} 2$, $P<0.001)$ and IL-17 (KD1 $<$ KD2, $P<0.001)$ significantly decreased after IVIG therapy. In comparison, there was an increase in Treg related FoxP3 (KD1 vs KD2, $P=0.006)$ and TGF- $\beta(\mathrm{KD} 1$ vs KD2, $P=0.175)$ expression. There was no significant difference in cytokine levels in Kawasaki patients with or without coronary artery lesions.

Conclusion: Kawasaki disease is associated with higher levels of IL-6, IL-17 and TGF$\beta$ cytokine expression. IVIG therapy led to a rapid decrease of Th17-related IL-6 and IL-17 expression and increased Treg related FoxP3 and TGF- $\beta$ expression. Overall, these results from this study may support that KD is an auto-immune like disease.

150

Influence of different forms of alpha-1 antitrypsin on cANCA mediated neutrophil activation

Surmiak, $\mathrm{M}^{1}$; Sanak, $\mathrm{M}^{1}$

II Department of Internal Medicine, Jagiellonian University Medical College, Krakow, Poland

Background: Granulomatosis with polyangiitis (GPA) is an infrequent, autoimmune disease with still unknown etiology. One of the symptoms characteristic for GPA is a serum presence of antineutrophil cytoplasmic antibodies with specificity for proteinase-3 (cANCA). By inhibition of PR3/ cANCA complex formation alpha-1 antitrypsin (A1AT) can prevent cANCA mediated neutrophil activation. Participation of A1AT in pathomechanisms of GPA can be confirmed by observation that patients with GPA and A1AT deficiency have worse course of the disease than patients with normal serum level of A1AT. However, the level of A1AT probably is not the main reason of this phenomenon. A1AT is an acute phase protein and it's level can be highly elevated during inflammation. The aim of this study was to analyze how different types of A1AT can affect cANCA mediated neutrophil activation.

Method: Neutrophils isolated from 12 healthy volunteers were stimulated with cANCA in the presence of native and polymerised A1AT. Influence of different forms 
of A1AT on cANCA mediated neutrophil's gene activation was analyzed by real-time PCR. Evaluation of influence of A1AT on cANCA/PR3 interaction and production of ROS by cANCA activated granulocytes was performed by flow cytometry. Concentration of NE/A1AT complexes was determined with ELISA.

Results: Experiments with A1AT showed that the native form of this protein can inhibit PR3/cANCA complex formation, as well as cANCA mediated neutrophil genes activation and ROS production. Higher concentration of NE/A1AT complexes was observed in supernatants from cells prestimulated with native A1AT.

Conclusion: Native A1AT can inhibit PR3/ cANCA interaction and genes activation/ ROS production in cANCA stimulated granulocytes. Poor inhibition properties of polymerised A1AT are probably caused by loss of antiprotease activity by polymerised form of protein. Inhibition of cANCA mediated neutrophil activation by A1AT is connected with enzymatic activity of plasma expressed PR3.

151

Periodic fever syndromes and autoinflammatory diseases: a case series from an Italian centre

Firinu, D; Murgia, G; Mura, MN; Peralta, MM; Torrazza, M; Frau, A; Manconi, PE; Del Giacco, SR Department of Medical Sciences 'M.Aresu', University of Cagliari, Monserrato, Italy

Background: Autoinflammatory diseases (AIDs) are a group of disorders with a heterogeneous clinical spectrum that is characterised by recurring episodes of fever and systemic inflammatory symptoms, affecting the serosal surfaces, joints, skin and eyes. Most of them are caused by mutations in specific genes that lead to the dysregulation of innate immune system. Within these disorders are included among AIDs of the bone, including chronic recurrent multifocal osteomyelitis (CRMO) - most common in children - and Synovitis, Acne, Pustulosis, Hyperostosis and Osteitis (SAPHO) syndrome, that is the most common in adults.

Method: We report the features of patients with AIDs observed at our department between 2009 and 2013.

Results: Fourteen patients were diagnosed as periodic fever. All cases did not satisfy criteria for familial Mediterranean fever (FMF) and had a negative screening for MEFV, TNFRSF1A and MVK genes mutations. Three of them had Periodic fever, Aphthous stomatitis, Pharyngitis, Adenitis (PFAPA) syndrome, two had atypical adult-onset Still's disease, and one patient had both periodic fever and Xlinked thrombocytopenia. Five cases were responsive to colchicine. Ten patients were diagnosed with SAPHO and one with CRMO. In the SAPHO group, chronic inflammatory disorders of the skin were observed in 9/10 patients, palmo-plantar pustulosis in $7 / 9$ and psoriasis vulgaris in $2 / 9$. Sternoclavicular osteitis was present in all cases, sacro-ileitis in 5/10 and Spondilodiscitis in 2/10 cases. Corticosteroids, common immunosuppressive drugs and pamidronate were unsuccessful in $9 / 10$ patients. They were treated with biological drugs, including anti-TNF $\alpha$ agents, antiIL1 agents and ustekinumab achieving total or partial remission.

Conclusion: Interestingly, all our patients with autoinflammatory diseases have a negative genetic screening and we observed a good response to colchicine. Furthermore, biological drugs showed to be effective in almost all SAPHO and CRMO cases.

\section{2}

Variation of alternative and classical complement pathways in ANCAassociated vasculitides

Djenouhat, $\mathrm{K}^{1}$; Ibsaine, $\mathrm{O}^{2}$; Yahi, $\mathrm{R}^{1}$; Benyahia, $\mathrm{L}^{3}$; Bedioune, $\mathrm{I}^{4}$

'Immunology, Institut Pasteur d'Algerie, Algiers, Algeria; ${ }^{2} \mathrm{CHU}$ Hussein Dey, Pesiatrics, Algiers, Algeria; ${ }^{3}$ Biogroupe Eucalyptus, Algiers, Algeria; ${ }^{4} \mathrm{CHU}$ Setif, Setif, Algeria

Background: Anti-neutrophil cytoplasmic autoantibody (ANCA)-associated vasculitides (AAV) is a group of disorders characterised by necrotizing small-vessel vasculitis. These immune complexes diseases are supposed activating classical complement pathway. The aim of the present work is to assess complement variation and its significance in AAV.

Method: 32 patients with AAV were included in this study and were stratified in the second step according to state's activity of disease. Complement investigation is based in exploration of Classical of complement including Antigenic dosages of C3 and $\mathrm{C} 4$ by nephelometry and factor B by Radial immunodiffusion (Binding Site, U.K.) and measurement of $\mathrm{C} 3 \mathrm{a}$ were detected by an enzyme-linked immunoassay kit (Quiedel, USA).

Results: Hypocomplementemia is observed in 13 among the 32 AAV patients $(40.6 \%)$. When dividing our population into 2 groups, 18 were in remission and 14 were in relapse phase. 9 of 14 patients in relapse and 6 of those in remission phase have activation of complement system. (64\% vs $33 \%, P<0.01$ ) However, alternative pathway with decreased of factor $B$ was activated in 4 cases among those with hypocomplementemia. Lectin pathway wasn't assessed in our work.
Conclusion: Results of this preliminary study shows that exploration of complement could be used as a parameter of monitoring AAV study of a pathogenic role for the alternative pathway in ANCA induced may help us to improve our understanding of the disease.

\section{3}

Evaluation of cytokine profiles in patients with chronic erosive vs non-erosive oral lichen planus

Drannik, $\mathrm{GN}^{1}$; Kurchenko, $\mathrm{Al}^{1}$; Rehuretska, $\mathrm{RA}^{1}$; DuBuske, $\mathrm{LM}^{2,3}$

National Medical University, Kiev, Ukraine; ${ }^{2}$ Immunology Research Institute of New England, Gardner, MA, United States; ${ }^{3}$ The George Washington University School of Medicine, Washington, DC, United States

Background: Oral lichen planus (OPL) is a serious medical and social problem. The incidence of erosive forms of OPL is continuously increasing promoting a risk of malignancy. IFN-g), IL-4, IL-10 and TGF$\beta$ reflecting Th1, Th2 and T-reg responses may impact development of chronic OLP. Methods: 97 patients aged 18 to 60 years were studied including 35 with erosive oral lichen planus, 32 with non-erosive oral lichen planus and 30 healthy control subjects of similar age. The levels of serum cytokines IFN- $\gamma$, IL-4, IL-10, and TGF- $\beta$ were determined by enzyme-linked immunosorbent assay ('Immunotech'; France).

Results: During relapse of erosive oral lichen planus there is a marked increase in select cytokines compared with control group levels including IL-4 $(52.1 \pm 2.3 \mathrm{pg} /$ $\mathrm{ml}$ ) having a 2.6- fold increase vs the healthy controls and IL-10 having a 1.6fold increase $(80.2 \pm 1.5 \mathrm{pg} / \mathrm{ml} \quad$ vs $49.5 \pm 3.2 \mathrm{pg} / \mathrm{ml})$. IFN- $\gamma(0.5 \pm 0.2 \mathrm{pg} /$ $\mathrm{ml}$ ) however is reduced 4.6- fold vs healthy controls. In chronic OLP there is an increase in IFN-g concentration by 2.0 fold, an increase of TGF- $\beta$ by 3.0 - fold and an increase of IL-4 $(27.2 \pm 1.2 \mathrm{pg} / \mathrm{ml})$ compared to healthy controls. In chronic OLP IL-4 indexes were diminished 1.9fold in comparison to OLP relapse and IL10 increased 1.1- fold compared to disease relapse $(P<0.05)$.

Conclusions: Acute relapse and chronic stages of lichen planus of the oral mucosa are characterised by immunological changes detectable in peripheral blood serum including increases in concentrations of IL-4, IL-10, TGF- $\beta$ and reduction in concentration of IFN- $\gamma$, characteristic of development of a recurrent inflammatory immune response. The balance among Th1 vs Th2 and T reg cytokines may determine whether oral lichen planus becomes a chronic persistent erosive disease. 


\section{Oral Abstract Session}

\section{OAS 27 - Atopic dermatitis and other skin conditions: mechanisms}

\begin{abstract}
154
Methylation of the aryl hydrocarbon receptor repressor may reduce the risk of eczema in children with a loss of function of filaggrin gene

Karmaus, $\mathrm{W}^{1}$; Ziyab, $\mathrm{AH}^{2}$; Sutter, $\mathrm{T}^{3}$; Zhang, $\mathrm{H}^{1}$; Lockett, $\mathrm{GA}^{4}$; Holloway, JW ${ }^{4}$; Ewart, $\mathrm{SE}^{5}$; Arshad, $\mathrm{HS}^{6}$

${ }^{1}$ Epidemiology, Biostatistics \& Environmental Health

University of Memphis, TN, Memphis, United States:

${ }^{2}$ Epidemiology and Biostatistics, University of South

Carolina, Columbia, MO, United States; ${ }^{3}$ Department of

Biological Science, University of Memphis, Memphis,

TN, United States; ${ }^{4}$ Faculty of Medicine, University Southampton, Southampton, United Kingdom; ${ }^{5}$ Large Animal Clinical Sciences, Michigan State University, East Lansing, MI, United States; ${ }^{6}$ The David Hide Asthma \& Allergy Research Centre, Newport, United Kingdom
\end{abstract}

Background: Coal tar and smoke exposure have been related to an increased expression of the filaggrin $(F L G)$ gene in case of genetic variants leading to a loss of function (LOF) of $F L G$. In particular in animal models, higher activity of the aryl hydrocarbon receptor repressor $(A H R R)$ gene has been demonstrated to mitigate $F L G$-LOF. To test an interaction of $A H R R$ and $F L G$ on the occurrence of eczema in humans, we examined genetic variants of $F L G$ and DNA-methylation of $A H R R$ in two generations (F1 and F2) of a birth cohort.

Method: At age 18, in 367 men and women of the original birth cohort (F1) and in 57 infant offspring (F2) we genotyped FLG variants (282del4, R501X and S3247X), measured DNA methylation of peripheral blood leukocytes at $>484000$ $\mathrm{CpG}$ sites, and collected information on the occurrence of eczema. The interaction of the AHRR methylome and FLG-LOF were tested using log-linear models.

Results: In the F1, 12.8\% of subjects had eczema at age 18 and $9.0 \%$ had FLG-LOF. Higher DNA methylation of the $\mathrm{CpG}$ site cg05575921 within AHRR (range of methylation: 42-91\%) was associated with a lower risk of eczema in F1 participants who had $F L G$-LOF $(P=0.03)$. In $F L G$ LOF cases, the median methylation of cg05575921 in participants with eczema was $73 \%$ and $81 \%$ in those without. Of the infants (F2, $n=57), 43.9 \%$ suffered from eczema in the first 12 months and $12.3 \%$ had a $F L G$-LOF genotype. In the F2 sample, the relative risk of $F L G$-LOF was also reduced in children with higher DNA methylation of $\operatorname{cg} 05575921$.

Conclusion: Methylation of the AHRR gene, in particular cg05575921, which is often related to exposure to xenobiotics, may mitigate the adverse effect that a loss of function of FLG genotype has on eczema.

\section{5}

Activity of natural moisturizing factor forming enzyme bleomycin hydrolase was impaired in the skin of atopic dermatitis

Morita, $\mathrm{K}^{1}$; Kitazawa, $\mathrm{H}^{1}$; Chiba, $\mathrm{T}^{1}$; Shibata, $\mathrm{M}^{2}$; Miyai, $\mathrm{M}^{2}$; Hibino, $\mathrm{T}^{2}$; Ohya, $\mathrm{Y}^{1}$

${ }^{1}$ Allergy, National Center for Child Health and Development, Setagaya-ku, Tokyo, Japan; ${ }^{2}$ Shiseido Research Center, Yokohama, Japan

Background: Skin barrier dysfunction is thought to play a pivotal role for the onset and exacerbation of atopic dermatitis (AD). Filaggrin is one of the key proteins consisting skin barrier function in stratum corneum (SC). It is produced in granular layer and degenerated into amino acids composing natural moisturizing factor (NMF) in SC. Decreased concentration of $\mathrm{NMF}$ is found to be a global feature of moderate-to-severe AD, but only $10-40 \%$ of $\mathrm{AD}$ patients carry mutation in FLG gene. We hypothesized that enzyme activity to form NMF from filaggrin might be decreased in the skin of AD patients. Bleomycin hydrolase $(\mathrm{BH})$ is an enzyme which produces NMF in the last stage of filaggrin degradation. In this study, we investigated the $\mathrm{BH}$ activity in the $\mathrm{SC}$ of patients with moderate-to-severe AD and healthy children for a comparison.

Method: By using tape stripping technique, we collected SC samples of lesional and nonlesion skin of 11 moderate-to-severe AD (SCORAD >25) children from 3 to 15 years of age. SC samples of age matched healthy children were also obtained. Enzyme assays of BH activity for these samples were carried out and the results of both groups were analyzed.

Results: The enzyme activity of $\mathrm{BH}$ in the lesional skin of AD was significantly lower than that of healthy children. (mean $\pm \mathrm{SD}$ : $34 \pm 20 \mathrm{pmol} / \mathrm{mg} / \mathrm{min}$ vs $99 \pm 135 \mathrm{pmol} /$ $\mathrm{mg} / \mathrm{min}, P=0.041$ ), but there was no difference between $\mathrm{BH}$ activity of nonlesional skin of $\mathrm{AD}$ and that of healthy children $(54 \pm 27 \mathrm{pmol} / \mathrm{mg} / \mathrm{min}$ vs $99 \pm 135 \mathrm{pmol} /$ $\mathrm{mg} / \mathrm{min}, P=0.809)$. BH activity of nonlesional skin of $\mathrm{AD}$ patients was higher than that of lesional skin $(54 \pm 27 \mathrm{pmol} / \mathrm{mg} /$ $\mathrm{min}$ vs $34 \pm 20 \mathrm{pmol} / \mathrm{mg} / \mathrm{min}, P=0.008$ ). Conclusion: Impaired activity of the enzyme $\mathrm{BH}$ in the eczematous skin of $\mathrm{AD}$ patient was found. BH might be a key player to protect the skin barrier from onset and/or exacerbation of eczema by forming NMF.

\section{6 \\ Characterisation of lesional skin biopsies and skin-derived $T$ cell cultures from atopic dermatitis and psoriasis patients \\ Poulsen, $\mathrm{BC}^{1,2,3}$; Dyring-Andersen, $\mathrm{B}^{1,4}$; Skov, $\mathrm{L}^{1}$; Aa- sted, $\mathrm{B}^{2}$; Thestrup-Pedersen, $\mathrm{K}^{1}$; Skak, $\mathrm{K}^{3}$; Poulsen, LK Department of Dermato-Allergology, Copenhagen University Hospital Gentofte, Hellerup, Denmark; ${ }^{2}$ Department of Veterinary Disease Biology, Faculty of Health and Medical Sciences, University of \\ Copenhagen, Copenhagen, Denmark; ${ }^{3}$ Department of Disease Pharmacology, LEO Pharma A/S, Ballerup, Denmark; ${ }^{4}$ Department of International Health, Immunology and Microbiology, Faculty of Health and Medical Sciences, University of Copenhagen, Copenhagen, Denmark}

Background: Atopic dermatitis (AD) is characterised by heavy infiltration of $\mathrm{T}$ cells in the skin. The aim of this study was to characterise and compare the phenotype of $\mathrm{AD}$ and psoriasis lesional skin biopsies and skin-derived $\mathrm{T}$ cell cultures.

Method: Lesional skin biopsies were collected from patients with AD [intrinsic (total $\operatorname{IgE}<200 \mathrm{kU} / 1$ ): $N=10$; extrinsic (total $\mathrm{IgE}>200 \mathrm{kU} / 1$ ): $N=7$ ], patients with psoriasis $(N=11)$ and healthy subjects $(N=8)$. One biopsy was used for microarray analysis and another was placed in media containing IL-2 $(1000 \mathrm{U} / \mathrm{ml})$ and IL-4 $(250 \mathrm{U} / \mathrm{ml})$, and incubated at $37^{\circ} \mathrm{C}$, to establish a skin-derived $\mathrm{T}$ cell culture. The cultures were analyzed by flow cytometry for surface markers and intracellular cytokines, once weekly after proliferation was established. Supernatants from cells 
cultured without IL-4 for $24 \mathrm{~h}$ were collected for cytokine analysis. Statistical method: Mann Whitney test, two-tailed.

Results: Proliferating T cell cultures were established from 8 out of 11 psoriasis biopsies and from 15 out of 17 AD biopsies. Psoriatic cultures tended to have higher levels of $\mathrm{IFN}^{+}$(10-fold) and IFN $\gamma^{+} \mathrm{t}$ bet $^{+}$(30-fold) cells compared to the AD cultures, and IFN $\gamma$ was also detected in the media from 3 of the psoriatic cultures, but not in any of the AD cultures. Also, the percentage of $\mathrm{CLA}^{+} \mathrm{CD} 8$ cells was higher than the level of $\mathrm{CLA}^{+} \mathrm{CD} 4$ cells in the AD cultures $(P=0.056)$, but not in the psoriatic cultures. Gene array showed 1576 differentially expressed genes (DEG) between $\mathrm{AD}$ and healthy skin, and 183 DEG between biopsies from extrinsic and intrinsic AD patients.

Conclusion: A tendency towards different IFN $\gamma$ profiles was found between $\mathrm{T}$ cell derived cultures from patients with $\mathrm{AD}$ and psoriasis. The transcriptomic analysis confirmed the notion of vastly different immunological mechanisms in $\mathrm{AD}$ and psoriasis.

\section{7 \\ Involvement of skin surface $\mathrm{pH}$ on barrier function in development of atopic dermatitis}

Tanaka, A; Jang, H; Matsuda, A; Amagai, Y; Matsuda, H Tokyo University of Agriculture and Technology, Tokyo, Japan

Background: Atopic dermatitis (AD) is a chronic inflammatory skin disease accompanied with impaired skin barrier function and T-helper type 2 (Th2) inflammation. Elevation of the skin surface $\mathrm{pH}$ has been reported in AD patients compared with healthy controls. However, involvement of high skin $\mathrm{pH}$ in development and severity of AD has not been well explored. Kallikrein 5 (KLK5), one of serine proteases produced by keratinocytes, is inactive in acidic $\mathrm{pH}$. Recent findings revealed that KLK5 associates with disruption of the skin barrier by inducing desquamation. Moreover, serine proteases may catalyze protease-activated receptor 2 (PAR2) on keratinocytes and neuronal cells, and stimulate cellular function. Since KLK5 displays its optimal activity in neutral $\mathrm{pH}$, there is a possibility that high skin $\mathrm{pH}$ may activate KLK5 resulting in proteolytic degradation of the skin barrier.

Results: Here, we show elevation of skin surface $\mathrm{pH}$ and increased expression of KLK5, protease-activated receptor 2 (PAR2), and thymic stromal lymphopoietin (TSLP) in the affected skins during spontaneous development of eczematous skin lesions in NC/Tnd mice, a model mouse for human AD. Alkalization of the skin of SPF NC/Tnd ice without AD increased trans epidermal water loss (TEWL) and scratching behavior, which are representing both skin barrier disruption and pruritus. Expression levels of KLK5, PAR2, and TSLP in the skin of $\mathrm{NC} /$ Tnd mice with alkalization were markedly elevated. On the other hand, mild acidification of the skin improved TEWL, scratching behavior, and clinical symptoms in atopic NC/Tnd mice. Production of KLK5, PAR2, and TSLP in the skin was suppressed in mice with mild acidification. In the current study, we first demonstrated that skin lesions were developed in SPF NC/Tnd mice by topical alkalization. In contrast, mild acidification reduced severity of skin lesions in conventional $\mathrm{NC} / \mathrm{Tnd}$ mice with spontaneous AD. Furthermore, we showed that elevation of skin $\mathrm{pH}$ activated KLK5 leading to the skin barrier disruption. KLK5 activation may involve in catalysis of PAR2 and TSLP production from keratinocytes. Finally, we found that topical mild acidification recovered skin barrier function of healthy volunteers who were treated with repeated tape stripping.

Conclusion: Our results provided that controlling skin $\mathrm{pH}$ will be beneficial care procedure for patients with atopic dermatitis.

\section{8 \\ Der $\mathbf{p} 2$ is a strong epicutaneous allergen even in the absence of TLR4}

Stremnitzer, $\mathrm{C}^{1}$; Manzano-Szalai, $\mathrm{K}^{2}$; Starkl, $\mathrm{P}^{1}$; Willensdorfer, $A^{2}$; Schrom, $S^{1}$; Singer, $J^{1}$; Reichart, $U^{3}$; Akira, $\mathrm{S}^{4}$; Jensen-Jarolim, $\mathrm{E}^{2}$

${ }^{1}$ Comparative Immunology and Oncology, Department of Pathophysiology and Allergy Research, Center of Pathophysiology, Infectiology and Immunology, Medical University of Vienna, Vienna, Austria;

${ }^{2}$ Comparative Medicine, Messerli Research Institute, University of Veterinary Medicine Vienna, Medical University of Vienna, University of Vienna, Vienna, Austria; ${ }^{3}$ Biomodels Austria, Institute of Animal Breeding and Genetics, University of Veterinary Medicine Vienna, Vienna, Austria; ${ }^{4}$ Department of Host Defense, Research Institute for Microbial Diseases, Osaka University, Osaka, Japan

Background: Der p 2, a major house dust mite allergen, has been identified as structural and functional homologue of MD-2 within the TLR4-MD2-CD14 complex and thereby can bind to LPS and activate TLR4 signaling. Recent studies in asthma models suggested, that TLR4 activation is crucial in sensitisation to Derp 2 and that TLR4-deficient mice are unresponsive to Der $\mathrm{p}$ 2. We investigated whether similar mechanisms are important for Der $p$ 2sensitisation via the skin.

Method: We established an epicutaneous sensitisation model to compare the response to recombinant Der $\mathrm{p} 2$ in combination with or without LPS between C57BL/6 WT and TLR4-deficient mice. Furthermore, we analyzed a possible adjuvant function of pretreatment with an exogenous cysteine protease.

Results: Repeated sensitisation with rDer p 2 induced high levels of allergen-specific $\operatorname{IgG} 1$ and $\operatorname{IgE}$ antibodies in both mouse strains. Addition of LPS increased the immune responses, but this effect was impervious of functional TLR4 expression. Moreover, TLR4 deficient mice developed even higher antibody titers than WT mice. Disruption of the skin barrier by the cysteine protease papain, a structural homologue to major house dust mite allergen

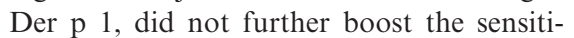
sation capacity of Der $\mathrm{p} 2$, but revealed a possible higher stability of LPS-complexed Der p 2 towards enzymatic degradation.

Conclusion: Der p 2 alone can cause a strong $\mathrm{T}_{\mathrm{H}}$ 2-biased response via the skin. In the presence of LPS this immune response can be enhanced, probably due to increased stability and prolonged encounter to immune cells of the LPS-complexed allergen. In contrast to data from lung models, Der p 2 sensitised via the skin in a TLR4-independent manner.

\section{9 \\ Experimental allergen immunotherapy with monomeric allergoid in a mouse model of atopic dermatitis}

Shershakova, N; Babakhin, A; Bashkatova, E; Kamishnikov, O; Shilovsky, I; Andreev, S; Khaitov, M Laboratory of Molecular Immunology, National Research Center - Institute of Immunology, Moscow, Russia

Background: Allergen immunotherapy (AIT) is an effective method and is widely used for treatment of allergic rhinitis and asthma but it's usage is limited in atopic dermatitis (AD). The purpose of this study was to evaluate the efficacy of monomeric allergoid (MA) in the treatment of mouse model of AD.

Method: AD experimental model was induced by the epicutaneous (EC) sensitisation with ovalbumin (OVA). Experimental AIT was performed by s.c. administration: (i) with non-modified OVA in escalating doses $(1,10,100$ and $1000 \mu \mathrm{g} /$ mice), four times for each dose; (ii) with OVA modified by succinitation (sOVA) twice in doses 120 and $600 \mu \mathrm{g} /$ mice, and three times in dose $1000 \mu \mathrm{g} /$ mice. Control group was treated with saline buffer. OVA-specific IgE, IgG1, IgG2a antibodies were assessed by ELISA. Cytokine production (IL-4, IL-5, IL-17, and IFN-g) by splenocytes upon OVA in vitro stimulation was detected by ELISA as well. 
Specimens of skin from OVA patch areas were removed for histological examination immediately after the last EC application with allergen.

Results: It was shown that AIT with both sOVA and OVA resulted in reduction of the anti-OVA IgE (also the ratio of IgG1/ $\mathrm{IgG} 2 \mathrm{a})$ as well as the level of IL-4. More- over, AIT with sOVA considerably increased the level of IFN-g. Histological analysis of skin samples from the sites of allergen application has shown that AIT improved the histological picture in terms of decreasing allergic inflammation manifestations compared to non-treated mice.
Conclusion: The modified sOVA is more effective for AIT treatment compared with non-modified allergen. Taken together, these data indicate that AIT with monomeric allergoids is a novel approach for the treatment of atopic dermatitis. 


\section{Oral Abstract Session}

\section{OAS 28 - Tools of monitoring AIT efficacy}

\begin{abstract}
160
Evanescent field-based fast measurements of allergen specific antibodies during SIT

Olzhausen, J1' Schawaller, $\mathrm{M}^{2}$; Wiki, $\mathrm{M}^{2}$; Akdis, $\mathrm{C}^{1}$; Jutel, $\mathrm{M}^{3}$; Crameri, $\mathrm{R}^{1}$; Rhyner, $\mathrm{C}^{1}$

${ }^{1}$ SIAF, Davos Platz, Switzerland; ${ }^{2}$ Davos Diagnostics, Davos Platz, Switzerland; ${ }^{3}$ Wroclaw Medical University, Wroclaw, Poland
\end{abstract}

An innovative optical evanescent fieldbased biosensor system has been developed for rapid, quantitative, and sensitive detection of multiple biomarkers. The novel optical readout system combines fluorescence detection with evanescent field excitation. An EVA-Reader instrument has been developed and its performance experimentally evaluated with different biochemical assays.

The EVA-System is composed of lowcost disposable polystyrene EVA-Chips equipped with the test-specific chemistry of choice, and a table-top, low-cost 'EVAReader' instrument with a small footprint. The EVA-biosensor system is a near patient testing device that allows fast and quantitative measurements nanomolar amounts of biomolecules with reduced wash and incubation steps, compared with standard ELISA methods.

Allergen-specific immunotherapy (SIT) represents the only curative treatment approach for allergic diseases. Increasing doses of an allergen are applied to an allergic patient with the aim to induce peripheral $\mathrm{T}$-cell tolerance and a shift from a $\mathrm{T}_{\mathrm{H}} 2$ to a $\mathrm{T}_{\mathrm{H}} 1 / \mathrm{T}_{\text {reg }}$-biased immune response. The aim of this study is the development of diagnostic assays to monitor changes in levels of allergen specific antibodies during SIT using the evanescent field technology. In a first EVA-Assay the major allergen responsible for cat allergy, Fel d1, was immobilized on the surface of an EVA- chip. The detection of Fel d1 specific IgE in human serum was achieved by an anti human $\operatorname{IgE}$ antibody labeled with fluorescent Allophycocyanine (APC) allowing detection and quantification within $10 \mathrm{~min}$. The detection limit of this assay is $0.17 \mathrm{kU}_{\mathrm{A}} / 1 \mathrm{Fel} \mathrm{d} 1 \mathrm{specific} \mathrm{IgE}$, which is as sensitive as an ELISA ( $\left.\mathrm{LOD}=0.14 \mathrm{kU}_{\mathrm{A}} / \mathrm{l}\right)$ performed with the same samples. Serums from allergic patients were measured by EVA, ELISA, and ImmunoСартм and the results obtained with all three detection systems showed significant correlations. In a second example of SIT, sera from 8 Mugwort pollen allergic patients undergoing SIT were measured in a direct assay using the major allergen Art v1. APC labeled anti human IgE or anti human IgG4 served as detection antibodies. During the first months of SIT an increase, and in a later stage, a decrease of Art v1 specific serum IgE levels were observed whereas Art v1 specific IgG4 starts to increase after the first month of SIT. These data obtained within $10 \mathrm{~min}$ show a decrease of the Art v1 specific $\operatorname{IgE} / \operatorname{IgG} 4$ ratio, commonly accepted as one indicator of successful SIT.

161

Molecular diagnosis may help to predict adverse reactions to immunotherapy in Spanish grass allergic patients

Sastre, J' ${ }^{1}$ Rodriguez, $F^{2}$; Campo, $\mathrm{P}^{3}$; Laffont, $\mathrm{E}^{4}$; Marin $\mathrm{A}^{5} ;$ Alonso-Diaz, $\mathrm{MD}^{6}$

${ }^{1}$ Allergy, Fundacion Jimenez Diaz, Tres Cantos, Spain; ${ }^{2}$ Hospital Valdecilla, Santander, Spain; ${ }^{3}$ Hospital Carlos Haya, Malaga, Spain; ${ }^{4}$ Hospital University Salamanca, Salamanca, Spain; ${ }^{5}$ ALK-Abello Madrid, Madrid, Spain; ${ }^{6}$ Hospital Fundacion Alcorcon, Alcorcon, Madrid, Spain

Background: To investigate if adverse reactions(ARs) during immunotherapy with a grass extract are related to the sensitisation of patients to different grass allergens.

Methods: 199 patients with rhinitis and/or asthma sensitised to grass pollen were recruited. The trial included 4 weeks updosing with 5-injections administered weekly (AVANZ ${ }^{\circledR}$ Phleum; ALK-Abelló). ARs were evaluated following EAACI guidelines. Explorative analysis of specific IgE to 8 grass allergens was performed by ISAC $^{\circledR}$. A value $>0.3$ ISU was considered positive. A comparison was done between patients suffering from AR during the build-up phase and the pattern of IgE sensitisation.

Results: Data from 177 patients were analyzed. A total of 432 ARs in $133(69 \%)$ patients were recorded; $57 \%$ local and $34 \%$ systemic. Most reactions were mild or moderate $(81 \%$ and $18 \%$ respectively). $98.5 \%$ of SRs were grade I or II. No grade IV reported.
The total number of ARs, local or systemic were significantly associated to the number of grass allergens the patients were sensitised to $(P=0.004, \quad P=0.003$, $P=0.01$, respectively). Sensitisation to $\mathrm{Phl}$ $\mathrm{p} 1+\mathrm{Phl}$ p5 or Phl p1 $+\mathrm{Phl}$ p5 + Phl p12 was significantly associated with a higher frequency of LR or SR ( $P=0.001$, both). Conclusion: Sensitisation to a higher number of grass allergens and sensitisation to $\mathrm{Phl}$ p1 $+\mathrm{Phl}$ p5 or Phl p1 $+\mathrm{Phl} \mathrm{p5}+\mathrm{Phl}$ p12 may potentially be considered risk markers to the development of adverse reactions during build-up phase of immunotherapy in a Spanish studied population. The clinical relevance of these explorative findings should now be confirmed in a suitably powered prospective trial.

\begin{tabular}{l}
\hline 162 \\
A study of house dust mite-specific \\
immunotherapy (HDM IT) reveals that \\
patient reported allergic \\
rhinoconjunctivitis symptom scores in \\
environmental exposure chamber (EEC) \\
are highly correlated with the sympto \\
in the field \\
Salapatek, $\mathrm{AM}^{1}$; Beattie, $\mathrm{E}^{1}$; Tang, $\mathrm{Z}^{1}$; Dabouz, $\mathrm{F}^{1}$; \\
Patel, $\mathrm{P}^{1}$ \\
${ }^{1}$ Inflamax Research, Mississauga, ON, Canada
\end{tabular}

Background: Study of HDM-IT in the field results in highly variable allergen exposure whilst the EEC allows controlled allergen exposure and effective screening.

Objective: To examine the correlation between allergy symptoms evoked by HDM in the EEC and symptoms experienced in the Field.

Method: A DBPC study was performed to investigate the efficacy, safety, and tolerability of intradermal, HDM IT. HDM allergic subjects $(n=172)$ were exposed to $10-120 \mathrm{ng} / \mathrm{m}^{3}$ HDM for $4 \mathrm{~h}$ in 3 pre-treatment, baseline EEC challenges and $3 \times 3$ post-treatment EEC challenges. Total Rhinoconjunctivitis Symptom Score (TRSS), a sum of 4 individual nasal (TNSS) and non nasal (TNNSS) symptoms were collected. A meta Pearson correlation analysis was conducted to evaluate field vs EEC symptoms scores, with $r$ values greater than 0.60 considered a strong correlation. The 
maximum value within 2 SDs from mean TRSS (TRSSmax) was also evaluated.

Results: Correlation analysis revealed that symptoms scores between the 2 models (Field and EEC) are strongly correlated, with $r$ values ranging from 0.54 to 0.79 (all $P<0.0001)$. Similarly, individual symptoms including itchy nose, itchy ear/palate, and ocular symptoms (itchy eyes, watery eyes, red eyes) are also strongly correlated, having $r$ values greater than 0.60 (all $P<0.0001)$.

Conclusion: These retrospective analyses demonstrate that rhinoconjunctivitis symptoms are highly correlated and statistically significant in the field and the EEC after IT treatment when there is a full spectrum of response to both placebo and active treatments, and thereby supports the EEC allergen challenge approach for pivotal IT testing in perennial allergies such as HDM allergy. Further, the EEC model allows for the screening of clinically symptomatic patients and makes possible to control and reliably conduct the required long-term follow-up testing to assess the duration of HDM IT efficacy and safety.

\section{3 \\ Dose-range finding of natural birch pollen exposure in patients with seasonal allergic rhinitis in the Fraunhofer Environmental Challenge Chamber}

Lueer, $\mathrm{K}^{1}$; Windt, $\mathrm{H}^{1}$; Schwarz, $\mathrm{K}^{1}$; Badorrek, $\mathrm{P}^{1}$; Sebastian, $\mathrm{R}^{1}$; Haefner, $\mathrm{D}^{2}$; Koch, $\mathrm{W}^{1}$; Krug, $\mathrm{N}^{1}$; Hohlfeld, $\mathrm{JM}^{1,3}$

Fraunhofer Institute for Toxicology and Experimental Medicine (ITEM), Hannover, Germany; ${ }^{2}$ Allergopharma GmbH \& Co. KG, Reinbek, Germany; ${ }^{3}$ Hannover

Medical School, Hannover, Germany

Introduction: Exposure to natural grass pollen in the Fraunhofer Environmental Challenge Chamber (ECC) has been validated and extensively applied for clinical development of investigational new drugs. Efficacy testing of immunotherapy using ECC needs allergen-specific exposures. The aim of this study was to test the safety and efficacy with dose-range finding and reproducibility of birch pollen challenge in subjects with seasonal allergic rhinitis in the Fraunhofer ECC.

Methods: In a monocenter, single-blind, placebo-controlled, six period dose escalation pilot study 18 subjects with seasonal allergic rhinitis and sensitisation to birch pollen were exposed to clean air (placebo) or birch pollen (Allergon AB, Sweden) at either 1000, 2000, 4000, and 8000 grains/ $\mathrm{m}^{3}$ for $4 \mathrm{~h}$ in the Fraunhofer ECC 7 days apart. The dose of 4000 grains $/ \mathrm{m}^{3}$ was repeated to investigate reproducibility. Efficacy (total nasal symptom score (TNSS), nasal flow rate, nasal secretion) and safety (adverse events, lung function) were assessed prior to and during the exposures. Results: While symptoms were absent prechallenge (TNSS $=0$ ), exposure to birch pollen increased TNSS (mean of last $2 \mathrm{~h}$ $\pm \mathrm{SD})$ to $3.5 \pm 0.2,4.0 \pm 0.2,4.3 \pm 0.2$, and $4.3 \pm 0.2$ at 1000, 2000, 4000, and 8000 grains $/ \mathrm{m}^{3}$, respectively, while air alone had little effect on TNSS (1.4 \pm 0.2$)$. Repeated exposure to 4000 grains $/ \mathrm{m}^{3}$ induced a mean TNSS of $4.3 \pm 0.3$ which was not statistically different compared to the same previous dose. Objective measures of nasal flow and nasal secretions were in line with clinical symptoms. Exposure to birch pollen was safe with no relevant change in $\mathrm{FEV}_{1}$.

Conclusion: Exposure to natural birch pollen at increasing concentrations in patients with allergic rhinitis in the Fraunhofer ECC was safe and induced symptoms of allergic rhinitis with a narrow doseresponse and evidence of saturation. A dose of 4000 grains $/ \mathrm{m}^{3}$ was effective, reproducible and can therefore be used in future clinical trials.

Supported by Allergopharma and Fraunhofer ITEM.

\begin{tabular}{l}
\hline 164 \\
Next generation sequencing and \\
antibody engineering in combination \\
defines different fates of allergen-specific \\
antibodies during specific \\
immunotherapy \\
Levin, $\mathrm{M}^{1} ; \mathrm{King}, \mathrm{JJ}^{2,3} ;$ Looney, $\mathrm{TJ}^{3} ;$ Hoh, $\mathrm{RA}^{3} ;$ Mari, $\mathrm{A}^{4}$; \\
Andersson, $\mathrm{M}^{5}$; Greiff, $\mathrm{L}^{5} ;$ Fire, $\mathrm{AZ}^{3,6} ;$ Boyd, SD ${ }^{3}$; \\
Ohlin, M ${ }^{1}$ \\
${ }^{1}$ Department of Immunotechnology, Lund University, \\
Lund, Sweden; ${ }^{2}$ Department of Biology, Stanford \\
University, Stanford, CA, United States; ${ }^{3}$ Department of \\
Pathology, Stanford University, Stanford, CA, United \\
States; ${ }^{4}$ Associated Centers for Molecular Allergology, \\
Rome, Italy; ${ }^{5}$ Otorhinolaryngology, Head and Neck \\
Surgery, Skåne University Hospital, Lund, Sweden; \\
${ }^{6}$ Department of Genetics, Stanford University, Stanford, \\
CA, United States
\end{tabular}

Background: Specific immunotherapy (SIT) is the only disease-modifying approach with potential to cure allergic disease. SIT may mediate this effect though modification of the antibody (Ab)-response against relevant allergens. The molecular details of the re-shaping of the allergenspecific $\mathrm{Ab}$ response during SIT are however not known.

Method: Genes encoding the heavy chain variable domain $(\mathrm{VH})$ of $\mathrm{Ab}$ of different isotypes (including $\mathrm{IgE}$ ) as found in peripheral blood B cells (PB-B) and in nasal biopsies taken from patients $(n=8)$ before and during the first year of SIT were sequenced on the Roche 454 (Titanium chemistry) Next Generation Sequencing
(NGS) platform. Genes encoding IgE with defined allergen specificity were isolated using phage display technology from combinatorial libraries generated from mRNA found in PB-B of the allergic donors 2 months after initiation of SIT. A bioinformatics pipeline was used to analyze the presence of sequences encoding these allergen-specific $\mathrm{Ab}$ in the different $\mathrm{PB}$ and nasal biopsy samples.

Results: 52 clonally unrelated, monoclonal IgE specific for 7 grass and tree pollen and mite allergens were selected from the $\operatorname{IgE}$ repertoires of subjects undergoing SIT. Some allergen-specific clone types were uniquely represented in samples obtained at 2 months after initiation of SIT, as determined by NGS. Others were, however, encoded by PB-B, often as differently mutated variants, before initiation of SIT and/or after 1 year of SIT. The same Ab clone types, encoding IgE, IgG or IgA, were also in several cases identified in nasal biopsies.

Conclusion: The utilisation of NGS and $\mathrm{Ab}$ library technology together allowed us to describe different fates of allergen-specific antibody clones in $\mathrm{PB}$ and in tissue affected by allergic disease during SIT. We envisage that this approach will be instrumental in defining potential differential outcomes of different types of SIT in terms of the molecular details of allergen-specific $\mathrm{Ab}$ responses.

\section{5 \\ Identification of a candidate biomarker predicting clinical benefit during allergen immunotherapy}

Caillot, N; Bouley, J; Jain, K; Lombardi, V; Bodo, V; Nony, E; Mariano, S; Chabre, H; Batard, T; Moingeon, $\mathrm{P}$ Stallergenes, Antony, France

Background: Allergen immunotherapy (AIT) is an efficient long-lasting effect treatment against respiratory allergies. As of today, AIT would take advantage from the identification of biomarkers (BMK) predicting clinical efficacy.

Method: Sera obtained from 82 grass-pollen allergic patients treated with grass-pollen tablets or placebo and regularly monitored in an allergen challenge chamber for 4 months were analyzed by comparative proteomics, i.e. 2-dimentional differential gel electrophoresis (2D-DiGE) and label-free mass spectrometry (MS). The potential role of a candidate biomarker in allergy physiopathology was subsequently studied in a mouse model of OVA-induced allergic asthma.

Results: 2D-DiGE analysis revealed posttranslational modification changes of a serum protein in active patients responding 
to treatment. Importantly, expression levels of these proteoforms correlated with clinical benefit in active group, but no such correlation was observed in placebo-treated patients. Enzymatic deglycosylation strategies combined with MS characterisation highlighted specific glycans affecting the protein separation pattern in $2 \mathrm{D}$ gels. The relevance of those glycan moieties to discriminate serum samples was addressed by quantifying glycopeptides in a label-free
MS approach. Interestingly, one glycoprotein was more abundant in sera of patients responding to AIT and its expression level was positively correlated with clinical benefit only in active group. Lastly, invalidation of the serum protein by RNA silencing increased airway hyper-reactivity and Th2 response for OVA-sensitised mice.

Conclusion: We identified a specific glycoform of a serum protein that allows predicting the patients with greater chances to benefit from AIT. Larger clinical cohorts are currently being tested to validate this potential predictive BMK of a positive clinical response to AIT. Investigations using human in vitro cellular models are currently conducted to decipher the function of this glycoprotein in regulating immune responses. 


\section{Oral Abstract Session}

\section{OAS 29 - New developments in the evaluation of occupational asthma}

\section{6 \\ Serum specific IgG response to toluene \\ diisocyanate (TDI) -tissue \\ transglutaminase conjugate in TDI-induced occupational asthmatics}

Pham, DL; Kim, MA; Yoon, MG; Lee, SI; Shin, YS; Park, HS

Department of Allergy and Clinical Immunology, Ajou University School of Medicine, Suwon, Korea

Background: Tissue transglutaminase (tTG) is a posttranslational modifying enzyme located in airway epithelial cells. A potential contribution of serum specific $\operatorname{IgG}(\mathrm{sIgG})$ to tTG in airway inflammation of toluene diisocyanate (TDI)-induced occupational asthma (OA) was suggested. We hypothesized that TDI exposure may modify tTG to induce airway inflammation. Therefore, we prepared TDI-tTG conjugate and detected serum specific antibodies in sera of TDI-OA patients.

Methods: We enrolled 99 TDI-OA patients, 76 asymptomatic exposed controls, 208 patients with non-occupational asthma and 74 unexposed controls for this study. TDI-tTG conjugate was prepared and confirmed by a native gel. Serum $\operatorname{sgG}$ and/or specific IgE (sIgE) antibodies to tTG, TDI-tTG, TDI-human serum albumin (TDI-HSA) conjugate, cytokeratin (CK) 19, and serum cytokine levels such as IL-8, TGF $\beta 1$ and TIMP-1 were measured by ELISA. We evaluated the level of IL-8 produced from airway epithelial cells (A549) treated with tTG in order to investigate the inflammatory effect of tTG and TDI-tTG.

Results: In TDI-OA group, the prevalence of serum sIgG to TDI-tTG $(17.2 \%)$ was higher than tTG $(11.1 \%)$, both of which were significantly higher than those of the three control groups $(P<0.05$ for all groups). TDI-exposed subjects with high levels of serum sIgG to TDI-tTG had a high prevalence of $\operatorname{sigG}$ to CK19, higher serum levels of TGF $\beta 1$ and TIMP-1. Both tTG and TDI-tTG increased the IL- 8 production from A549 cells dose-dependently. Conclusion: The findings suggest that TDI exposure in workplace binds to tTG to form a conjugate that can induce serum specific $\operatorname{IgG}$ antibody production, airway inflammation and airway remodeling in TDI-OA patients.

167

Allergenic profile of baker's asthma patients using microarray

Gomez-Casado, $\mathrm{C}^{1}$; Campo, $\mathrm{P}^{2}$; Garrido-Arandia, $\mathrm{M}^{1}$; Pereira, $C^{3}$; Palacin, $A^{1}$; Rivas, $L^{3}$; Catarino, $M^{4}$; Aranda $A^{5}$; Macias, $L^{5}$; Armentia, $A^{6}$; Quirce, $S^{7}$; Blanca, $M^{2}$; Diaz-Perales, $A^{1}$

${ }^{1}$ Centre for Plant Biotechnology and Genomics (UPMINIA), Madrid, Spain; ${ }^{2}$ Allergy Unit, IBIMA, Regional University Hospital of Malaga, UMA, Malaga, Spain; ${ }^{3}$ Molecular Evolution Laboratory, Astrobiology Centre (INTA-CSIC), Madrid, Spain; ${ }^{4}$ Faculty of Pharmacy, University of Lisbon, Lisbon, Portugal; ${ }^{5}$ Research Laboratory, IBIMA, Regional University Hospital of Malaga, UMA, Malaga, Spain; ${ }^{6}$ Allergy Service, Rio Ortega University Hospital, Valladolid, Spain; ${ }^{7}$ Allergy Service, La Paz University Hospital, Madrid, Spain

Background: Patients with baker's asthma recognise several allergens identified in the water/salt-soluble fraction of wheat flour. The aim of our study was to characterise the allergenic profiles of baker's asthma patients from three different regions in Spain by using a panel of wheat allergens purified from natural sources and printed on a protein microarray.

Method: Forty five patients from 3 regions in Spain (Madrid $n=17$, Malaga $n=10$, Valladolid $n=18$ ) with a consistent history of baker's asthma, positive results to skinprick test and bronchial challenge with wheat flour were recruited. Twelve wheat allergens (WDAI-0.19 and WDAI-0.53, WTAI-CM1, WTAI-CM2, WTAI-CM3, WTAI-CM16,WTAI-CM17, Tri a 14, profilin, $\omega$-5-gliadin, Tri a Bd 36 and Tri a TLP) were purified and applied on epoxyactivated glass using a MicroGrid II TAS arrayer. The IgE binding of each allergen spot was calculated as the final fluorescence intensity, measured by GenePixTM software. The Ethics Committee of each hospital approved the study.

Results: WTAI-CM16 and Tri 14 were defined as the most prevalent allergens (54 and $45 \%$ on average, respectively) covering a total of $64 \%$ of the baker's asthma population. On the other hand, $\omega$-5-gliadin and Tri a $\mathrm{Bd} 36$ were recognised by less than $10 \%$ of the baker's population.
Conclusion: The highest prevalence of $\operatorname{IgE}$ binding was observed for WTAI-CM16 $(54 \%)$ and Tri a $14(45 \%)$, with $64 \%$ of patients with baker's asthma that recognised at least one of these markers.

\section{8 \\ Baker's asthma: inflammatory markers and polymorphisms in occupational exposure to flour dust \\ Marraccini, $\mathrm{P}^{1}$; Cantone, $\mathrm{L}^{2}$; Marsili, $\mathrm{C}^{3}$; Leghissa, $\mathrm{P}^{4}$; Santini, $\mathrm{M}^{4} ;$ Bancone, $\mathrm{C}^{4}$; Mosconi, $\mathrm{G}^{4}$; Barretta, $\mathrm{L}^{5} ; \mathrm{Pa}$ - trini, $L^{1}$; Previdi, $M^{1}$ \\ ${ }^{1}$ Medicina Preventiva, IRCCS Fondazione Ca Granda Policlinico, Milano, Italy; ${ }^{2}$ Scienze Cliniche e di \\ Comunità, Università degli Studi di Milano, Milano, Italy; ${ }^{3}$ Dottorato in Medicina del Lavoro, Università degli Studi di Milano, Milano, Italy: ${ }^{4}$ U.S.C. Medicina del Lavoro, Azienda Ospedaliera Papa Giovanni XXIII, Bergamo, Italy; ${ }^{5}$ Medicina Preventiva, Università degli Studi di Milano, Milano, Italy}

Background: It is worth notion that flour dust is pro-inflammatory and can determine both non-allergic airway inflammation and enhancement of allergen-mediated airway. Aim of the study was the clinical evaluation of exposed workers in relation to some inflammatory markers and genetic profiles related to CD14 and TLR4 polymorphisms.

Method: Three groups of 167 subjects (mean age 41 years), were so distributed: 63 healthy subjects, 45 atopic and 59 suffering from occupational asthma/rhinitis. Evaluation of fractional expired nitric oxide (FeNO) (NIOXMINO Aerocrine AB Sweeden) and serum cytokines, such as IL6, IL8 and TNF- $\alpha$ were performed (ELISA test R\&D Systems - USA) other than the clinical assessment of the workers. DNA in blood samples was amplified by PCR using specific primer pairs for the two loci containing the TLR4 gene $(+896)$ rs4986790 and the CD14 gene (-159) rs2569190 polymorphisms.

Results: IL6 values resulted high in atopic and in subjects with professional respiratory symptoms; a statistical difference was observed between atopic and healthy workers $(P<0.05)$. IL8 resulted higher in workers with rhinitis and baker's asthma than in other groups $(P<0.005)$. On the contrary increase of TNF- $\alpha$ was observed 
in healthy subjects $(P<0.003)$. FeNO increased in subjects with occupational asthma/rhinitis $(P<0.001)$, and a statistically significant correlation with IL8 was observed $(P<0.0001)$. No differences were detected for the TLR4 polymorphisms, although CD14 polymorphisms were differently expressed $(P<0.06)$.

Conclusion: In workers exposed to flour dust the increase of cytokines is probably related to an inflammatory stimulus and might be assumed as a defensive response either in atopic or healthy workers, suggesting their use as markers of exposure. Moreover, FeNO can be used as a monitoring parameter of inflammation, related to IL8, which is responsible for neutrophil recruitment into the lung. Genotype CD14$159 \mathrm{CC}$ seems to prevent the development of occupational allergy due to flour dust.

\section{9 \\ Occupational respiratory allergies in bakery workers and new diagnostic techniques: a study of the exhaled breath condensate metabolomic profile}

Olivetti, $\mathrm{J}^{1}$; Sabbadini, $\mathrm{MG}^{1}$; Colombo, $\mathrm{G}^{1}$; Ferraioli, $\mathrm{E}^{2}$, Carcano, $\mathrm{M}^{2}$; Brambilla, $\mathrm{M}^{3}$; Brambilla, $\mathrm{P}^{3}$; Mascagni, $\mathrm{P}^{2}$ ${ }^{1}$ Unit of Medicine and Clinical Immunology, Università Vita-Salute San Raffaele, Milan, Italy; ${ }^{2}$ Clinical Unit of Occupational Health, Hospital of Desio, Desio, Italy; ${ }^{3}$ University Department of Laboratory Medicine, Hospital of Desio - University of Milano Bicocca, Desio, Italy

Background: Baker's asthma is a frequent invalidating pathology among bakery and pastry workers. In this study we compared more sophisticated diagnostic techniques, such as the study of the metabolomic profile of the lungs, with traditional tests and we evaluated the new techniques for the first time in this background.

Method: The evaluation was performed in 11 asthmatic patients, in 20 asymptomatic subjects who were previously exposed to flour and in 10 healthy non-bakers healthy subjects. All the subjects underwent complete allergological evaluation (anamnesis, physical examination, skin prick tests and specific IgE). We also performed pulmonary function tests and fractional exhaled nitric oxide $\left(\mathrm{F}_{\mathrm{E}} \mathrm{NO}\right)$ measurement before and after exposure to flour. Exhaled breath condensate was collected with Turbo DECCS System in all subjects. Then, the metabolomic analysis was performed with High Performance Liquid Chromatography, Mass Spectrometry and analyzed with dedicated software.

Results: Traditional diagnostics effectively detects allergic reactivity against the allergens considered in the study. Functional pulmonary tests proved useful in the diagnostic workup of patients with occupa- tional asthma, especially for what concerns the use of $\mathrm{F}_{\mathrm{E}} \mathrm{NO}$ as an indicator of inflammation in affected patients. The observation of the metabolomic profiles has pointed out significantly different response patterns between affected patients and the other two control groups, which show homogeneous results.

Conclusion: The pulmonary metabolomics profile clearly identifies the lung environment of affected patients, thus allowing detecting molecules, such as aldehydes, that are possibly involved in the pathogenesis of baker's asthma. The role of these newly identified molecules needs to be confirmed by further analysis that could open the way to new diagnostic possibilities and more effective, targeted and customized therapeutic strategies.

\section{0 \\ Bioaerosol exposure, allergy and respiratory symptoms in the Norwegian crab industry}

Thomassen, $\mathrm{MR}^{1,2}$; Bang, $\mathrm{BE}^{1,3}$; Kramvik, $\mathrm{E}^{1}$; Hegseth, $\overline{\mathrm{MN}}^{1}$; Holm, M-H ${ }^{1}$; Larsen, $\mathrm{M}^{1}$; Olsen, $\mathrm{RKF}^{1}$; Andorsen, $\mathrm{GS}^{1}$; Fuhr, $\mathrm{T}^{4}$; Eduard, $\mathrm{W}^{5}$; Madsen, $\mathrm{AM}^{6}$; Kamath, $\mathrm{SD}^{7}$; Lopata, $\mathrm{AL}^{7}$; Aasmoe, $\mathrm{L}^{1}$

${ }^{1}$ University Hospital of North Norway, Occupational and Environmental Medicine, Troms $\varnothing$, Norway;

${ }^{2}$ Department of Community Medicine, UiT The Arctic University, Troms $\varnothing$, Norway; ${ }^{3}$ Department of Medical Biology, UiT The Arctic University of Norway, Troms $\varnothing$, Norway; ${ }^{4}$ Ear, Nose and Throat, University Hospital of North Norway, Troms $\emptyset$, Norway: ${ }^{5}$ National Institute of Occupational Health, Oslo, Norway; ${ }^{6}$ The National Research Centre for the Working Environment, Copenhagen, Denmark; ${ }^{7}$ Centre for Biodiscovery and Molecular Development of Therapeutics, James Cook University, Townsville, Old, Australia

Background: Fishing and processing edible crab (Cancer pagurus) in Norway has existed for over 100 years and the explosion of king crab (Paralithdodes camtschaticus) the last decade has increased the crab based industrial activity on shore. Previous studies in the seafood industry found an increased prevalence of general and work related respiratory symptoms. In Canada and Alaska, crab processing has been associated with occupational asthma and asthma-like symptoms. Bioaerosols are a common exposure for seafood workers in processing plants.

The aims of this project is to characterise occupational exposure to bioaerosols, the prevalence of general and work related respiratory symptoms, and $\operatorname{IgE}$ to crab among processing workers.

Method: Personal air samplers collected bioaerosols produced during crab processing. Workers and a non -exposed control group answered questionnaires about general and work related respiratory symptoms. Blood samples were collected and analyzed for crab IgE. Skin prick testing was performed on exposed workers with extracts made in our lab of the king and edible crabs' raw meat, cooked meat, intestines and shell.

Results: Total protein, tropomyosin and endotoxins were found in all air samples collected, with higher levels than in most other studies in the seafood industry. Some samples also showed NAGase activity. The prevalence of general respiratory symptoms and symptoms during work was higher in crab workers compared to non-exposed controls.

Specific $\operatorname{IgE}(>0.35 \mathrm{kU} / \mathrm{l})$ to crab was found in 11 of 113 king crab workers and 10 of 78 edible crab workers. Skin prick tests showed a reaction to crab in 4 of 40 king crab workers and 15 of 83 edible crab workers.

Conclusion: The workers are exposed to biologic active agents in the bioaerosols produced during crab processing. They also report a higher prevalence of respiratory symptoms than non exposed workers. Several of the workers had elevated IgE to crab and tested positive on skin prick tests.

\section{1 \\ Molecular and immunological approaches in quantifying the air-borne food allergen - tropomyosin}

Kamath, $\mathrm{SD}^{1}$; Thomassen, $\mathrm{MR}^{2}$; Saptarshi $\mathrm{SR}^{1}$; Aasmoe, $\mathrm{L}^{2}$; Bang, $\mathrm{BE}^{2}$; Lopata, $\mathrm{AL}^{1}$

${ }^{1}$ Centre for Biodiscovery and Molecular Development of Therapeutics, James Cook University, School of Pharmacy and Molecular Sciences, Townsville, Qld, Australia; ${ }^{2}$ University Hospital of North Norway, Tromsoe, Norway

Background: Tropomyosin is the major shellfish allergen present in a variety of consumed shellfish species such as crustaceans and molluscs. In the seafood industry, workers are constantly exposed to bioaerosols containing air-borne food allergens created during processing activities through inhalation, which could lead to allergic sensitisation. The aim of this study was to develop a sensitive immunoassay for specific detection and quantification of aerosolized food allergen, tropomyosin in the working environment of the shellfish processing industry.

Method: Crustacean tropomyosin-specific polyclonal rabbit antibodies were generated and purified using immuno-affinity isolation and employed as the capture system. Recombinant prawn tropomyosin was expressed in Escherichia coli and purified using two-step chromatography and used as an allergen standard for the validation of the immunoassay. For quantification of aerosolized tropomyosin, air samples were collected from the personal breathing zones 
of 80 workers involved in edible and king crab processing activities.

Results: The capture system was able to selectively detect crustacean tropomyosin but not vertebrate (fish or pig) tropomyo$\sin$. The limit of detection of the developed immunoassay was 60 picogram $/ \mathrm{m}^{3}$ and limit of quantitation (LOQ), 100 picogram/ $\mathrm{m}^{3}$. Immunoassay validation was based on linearity $\left(R^{2} 0.999\right)$, spike recovery test $(78.8 \pm 6.5 \%)$, intra-assay $\mathrm{CV}(9.8 \%)$ and inter-assay CV (11\%). Using the immunoassay, work activities leading to low, medium or high allergen exposures were identified.

Conclusion: We describe the development and application of a highly sensitive immunoassay for specific quantification of the major food allergen, tropomyosin. This novel approach for detecting air-borne food allergens could be easily modified for use with other food sources thus assisting in identification of high-risk exposure areas in the food industry. 


\section{Oral Abstract Session}

\section{OAS 30 - Animal studies in asthma}

172

Role for IL-33 in the induction of immunoglobulin production after inhalation of HDM in mice

Canbaz, $D^{1}$; Utsch, $L^{1}$; Logiantara, $A^{1}$; van Ree, $R^{1,2}$; $\overline{\text { van Rijt, } L^{1}}$

${ }^{1}$ Experimental Immunology, Academic Medical Center, Amsterdam, The Netherlands; ${ }^{2}$ Otorhinolaryngology,

Academic Medical Center, Amsterdam, The

Netherlands

Background: Sensitisation to house dust mite (HDM) is considered to be dependent on the interplay between dendritic cells (DCs) and epithelial cells. IL-33, released by HDM exposed epithelial cells, activate airway DCs which consecutively prime Th2 cells. Here we investigate the respective role of IL-33 and DCs in sensitisation to HDM.

Method: To study the DC driven immune response, bone marrow derived DCs were pulsed with an endotoxin low 'LoTox' HDM extract (HDM DCs). Naïve mice received HDM DCs intratracheally (i.t.) and were rechallenged intranasally (i.n.) with HDM extract 10 days later. To investigate the need of $\mathrm{B}$ cells for free antigen for immunoglobulin production, additional HDM simultaneous with HDM DCs was administered i.t. at day 0 . To investigate an IL-33 driven immune response, mice were sensitised with HDM or HDM DCs with or without IL-33 i.t. at day 0 and were challenged i.n. at day 10 with HDM. At day 13, eosinophil recruitment, Th2 cytokine production, total IgE, HDM specific IgG1 and airway histology were analyzed.

Results: HDM DCs induced an eosinophilic airway inflammation, mucus production and IL-4, IL-5, IL-13 and IL-17A production compared with sensitisation with unpulsed DCs but an antibody response was completely lacking. Addition of free HDM was not sufficient to induce an antibody production. Only when IL-33 was simultaneously administered with HDM 'LoTox' pulsed DCs and/or free 'LoTox'HDM, HDM specific IgG1 and total $\mathrm{IgE}$ were induced.

Conclusion: These results demonstrate that HDM pulsed DCs were able to drive a Th2 response but that IL-33 was needed to induce a humoral immune response to inhaled HDM.
173

Notch signaling pathway intervention opens new therapeutic opportunities in allergic asthma

Kleinjan, $A^{1}$; Van Nimwegen, $M^{1}$; De Bruijn, MJW $^{1}$. Bergen, $\mathrm{I}^{1} ;$ Hoogsteden, $\mathrm{HC}^{1}$; Amsen, $\mathrm{D}^{2}$; Hendriks, RW ${ }^{1}$ ${ }^{1}$ Pulmonary Medicine, Erasmus Medical Center, Rotterdam, The Netherlands; ${ }^{2}$ Sanquin, Amsterdam, The Netherlands

Background: Expression of the Notch ligand Jagged on activated dendritic cells (DCs) is thought to contribute to T helper 2 (Th2) differentiation. DC-T cell interaction induces Notch signaling. Activation of Notch induces translocation of the Notch intracellular domain (NICD) from the cytoplasma to the nucleus, where it binds to RBP-J and MAML. This nuclear complex is the main effector of Notch and controls IL-4-independent differentiation of Th2 cells through direct Gata3 transcriptional activation.

In this report, we used $\mathrm{T}$ lymphocytespecific deletion of RBPJ, to investigate whether Notch signaling plays a crucial role in the induction of allergic asthma in mice. Assembly of the active transcriptional RBPJ-containing nuclear complex can be prevented by SAHM1, which is a hydrocarbon-stapled peptide that displaces MAML1 and thereby blocks Notch transcription activity. We evaluated therapeutic effects of SAHM1 on allergic airway inflammation in mice.

Method: T-cell specific conditional RBPJ knock-out mice and WT littermates were studied in asthma models, based on sensitisation and challenge either with

1. house dust mite (HDM), or

2. GM-CSF-cultured bone marrowderived DCs.

Bronchial hyperreactivity (BHR), serum IgE and bronchial alveolar lavage (BAL) cells (eosinophils, $\mathrm{T}$ cell cytokine content) were quantified in the mice. Therapeutic intervention with SAHM1 was performed in HDM asthma models.

Results: Upon HDM-treatment, WT animals developed asthma as confirmed by BHR, eosinophilic airway inflammation and elevated numbers of $\mathrm{T}$ cells expressing IFN $\gamma$, IL-4, IL-5 and IL-13 in BAL. In contrast, RBPJ $\mathrm{KO}$ animals failed to develop any signs of BHR or eosinophilic inflammation. Serum IgE, Th1- or Th2- cytokines were not induced. Because IL-17producing $\mathrm{T}$ cells were induced, RBPJ-deficiency did not abrogate general $\mathrm{T}$ cell activation. Sensitisation and challenge with Jagged-expressing DCs induced airway inflammation in WT mice, but significantly less in RBPJ KO mice. SAHM1 treatment during sensitisation and challenge resulted in a strong reduction in eosinophilic inflammation. SAHM1 showed a doseresponse curve with an optimal dose of $3 \mu \mathrm{g}$. Additional experiments showed effective SAHM1 treatment both during challenge phase and resolution phase.

Conclusion: Jagged-Notch signalling is required for the induction of asthma in mice. Moreover, therapeutic intervention in the Notch signaling pathway by SAHM1 is an interesting new topical treatment opportunity in asthma.

\section{4}

Sequential effect of subcutaneous immunotherapy for birch pollen allergy on Th2 cell suppression and attenuation of airway hyperreactivity in a murine asthma model

van Rijt, L ${ }^{1}$; Logiantara, $A^{1}$; Utsch, $L^{1}$; Canbaz, $D^{1}$; Opstelten, $\mathrm{D}-\mathrm{J}^{2}$; van der Kleij, $\mathrm{H}^{2}$; van Ree, $\mathrm{R}^{1,2,3}$

${ }^{1}$ Academic Medical Center, Experimental Immunology, Amsterdam, The Netherlands; ${ }^{2} \mathrm{HAL}$ Allergy BV, Leiden, The Netherlands; ${ }^{3}$ Otorhinolaryngology, Academic Medical Center, Amsterdam, The Netherlands

Background: The suppression of Th2 cytokine production by allergen specific Th2 cells is considered to be critical for the suppression of allergic symptoms by subcutaneous immunotherapy (SCIT). The aim of this study was to develop a mouse model for birch pollen (BP) allergy that can be used as a preclinical model to improve allergen immunotherapy and to elucidate the underlying mechanisms that contribute to improvement of clinical symptoms.

Method: Mice with birch pollen induced allergic airway inflammation received 2, 4, 6 or 8 weekly subcutaneous immunotherapy injections with birch pollen extract adsorbed to alum. The effect of the number of injections and an increasing dose of birch pollen extract adsorbed to an equal concentration alum on the amelioration of airway inflammation, Th2 cytokine production, immunoglobulin production and 
airway hyperresponsiveness was determined.

Results: After two injections, Th2 cytokine production, eosinophil recruitment, peribronchial inflammatory infiltrates were suppressed and BP specific immunoglobulins were induced but this did not prevent airway hyperresponsiveness. Only after eight injections, airway hyperresponsiveness was suppressed which was accompanied by an increasing titer of BP $\operatorname{IgG} 2 \mathrm{a}$ while other immunological parameters were stably suppressed after 2 injections.

Conclusion: This study showed that amelioration of airway hyperreactivity was delayed compared with suppression of Th2 mediated eosinophilic airway inflammation by BP SCIT. Induction of sufficient protective BP immunoglobulins might be involved.

\section{5 \\ Aerobic exercise training reduces asthma phenotype via inhibition of cytokines production in lymphatic organs mediated by $\mathrm{P} 2 \mathrm{X7}$ receptor}

Greiffo, $\mathrm{FR}^{1}$; Custodio, RWA ${ }^{1}$; Oliveira, $\mathrm{NCR}^{1}$; Cipriani, $\mathrm{F}^{1}$; Damaceno-Rodrigues, $\mathrm{NR}^{2}$; Sousa, $\mathrm{ASA}^{1}$; Oliveira, $\mathrm{ARA}^{1}$; Pereira, $\mathrm{PR}^{1}$; Oliveira-Junior, $\mathrm{MC}^{1}$; Albertini, $\mathrm{R}^{1}$; Ligeiro de Oliveira, $\mathrm{AP}^{1}$; Vieira, $\mathrm{RP}^{1}$

${ }^{1}$ Nove de Julho University, Sao Paulo, Brazil;

${ }^{2}$ University of Sao Paulo - School of Medicine, Sao Paulo, Brazil

Background: Investigate the effects of aerobic exercise training (AET) in two experimental models of asthma on pulmonary inflammation and on lymphatic organs activation and the role of $\mathrm{P} 2 \mathrm{X} 7$ receptor in this response.

Method: Adult $\mathrm{C} 57 \mathrm{Bl} / 6$ and $\mathrm{BALB} / \mathrm{c}$ mice ( $n=5 /$ group) were distributed in Control, Exercise, Asthma and Asthma+Exercise groups. AET was performed in a treadmill, $5 \times /$ week, $1 \mathrm{~h} /$ session, $60 \%$ of maximal exercise capacity, during 4 weeks after the establishment of allergic airway inflammation. The ovalbumin and also house dust mite model of asthma was used. Under anesthesia, the total and differential cell number of leukocytes in the bronchoalveolar lavage (BAL), the levels of IL-5, IL-10 and IFN-gamma on BAL, serum and on the supernatant of $1 \times 106$ cells obtained from mediastinal lymph nodes, spleen and thymus were analyzed. The number of eosinophils in the airways wall and the collagen content and the mucus production in the airways wall was analysed. The extracellular ATP levels in BAL and the expression of $\mathrm{P} 2 \mathrm{X} 7$ receptor in the lungs and in the cells obtained from mediastinal lymph nodes, spleen and thymus were analyzed by western blotting.
Result: AET in asthma group (for both ovalbumin and HDM model of asthma) significantly reduced the number of total cells $(P<0.01)$ and eosinophils $(P<0.01)$ in BAL as well as the levels of IL-5 in BAL $(P<0.01)$, serum $(P<0.01)$ and in the supernatant of mediastinal lymph nodes $(P<0.01)$, spleen $(P<0.01)$ and thymus $(P<0.01)$, while increased the levels of IL-10 in BAL $(P<0.01)$, serum $(P<0.01)$ and in the supernatant of mediastinal lymph nodes $(P<0.01)$, spleen $(P<0.01)$ and thymus $(P<0.01)$. The results also demonstrated that AET reduced the levels of extracellular ATP in $\mathrm{BAl}$ and that AET reduced the expression of $\mathrm{P} 2 \mathrm{X} 7$ receptor in the lungs.

Conclusion: Aerobic exercise training reduces asthma phenotype by reducing lymphatic organs activation and these results seem to be, at least partially, by inhibition of $\mathrm{P} 2 \mathrm{X} 7$ receptor.

\section{6}

C10 exhibits a strong anti-inflammatory activity in a Th2 driven asthma model

Mascarell, L; Airouche, S; Jain, K; Nony, E; Baron-Bodo, V; Moingeon, $\mathrm{P}$

Research and Pharmaceutical Development,

Stallergenes, Antony, France

Background: Besides its well-known role in complement activation, we recently identified $\mathrm{C} 1 \mathrm{Q}$ as a pan-regulatory dendritic cell (DCreg) marker. Specifically, C1Q expression was increased in peripheral blood mononuclear cells from grass pollen allergic patients exhibiting clinical benefit during allergen immunotherapy. Herein, we further investigated the potential role for $\mathrm{C} 1 \mathrm{Q}$ in downregulating allergic inflammation.

Method: The effect of C1Q was studied in a murine asthma model. Ovalbumine (OVA) sensitised BALB/c mice received an intraperitoneal injection of C1Q (at doses of either $10 \mu \mathrm{g}, 50 \mu \mathrm{g}$ or $100 \mu \mathrm{g}$ ) before each of the four aerosol OVA challenges. As controls, groups of OVA-sensitised mice received either PBS, dexamethasone (DEX) or heat-denatured C1Q. Airway hyperresponsiveness (AHR), inflammatory cell infiltration [i.e. eosinophils and type 2 innate lymphoid cells (ILC2)] in bronchoalveolar lavages (BALs), Th2 cytokine production by OVA-specific $T$ cells in the lungs and seric OVA-specific IgE production were assessed in those animals.

Results: In a dose dependent manner, C1Q therapy induced a significant reduction in AHR as assessed by whole body plethysmography. Invasive monitoring of lung function in C1Q treated animals confirmed a significant increase in pulmonary resis- tance as well as a marked decrease in dynamic compliance. Additionally, a significant reduction in eosinophil and ILC2 infiltrates in BAL fluids as well as a decrease in Th2 cytokine secretion by OVA-specific $T$ cells was observed in mice receiving $\mathrm{C} 1 \mathrm{Q}$. $\mathrm{C} 1 \mathrm{Q}$ had no significant impact on seric OVA-specific IgE. Importantly, C1Q but not its heat-denatured form, was as efficient as DEX to downregulate clinical and biological signs associated with allergic asthma.

Conclusion: C1Q exhibits strong antiinflammatory activity in a Th2-driven asthma model. These findings draw attention on C1Q as a potential molecule for treating type I allergies in humans.

\section{7 \\ Transcutaneous sensitisation and bronchial challenge Induce IL-17A- mediated neutrophilia and smooth muscle contraction in a house dust mite model of asthma}

Chesné, $\mathrm{J}^{1,2}$; Braza, $\mathrm{F}^{1,2,3}$; Chadeuf, $\mathrm{G}^{1}$; Cheminant, M-A ${ }^{1}$; Lair, $D^{1}$; Brouard, $\mathrm{S}^{3}$; Loirand, $\mathrm{G}^{1}$; Sauzeau, $\mathrm{V}^{1}$. Magnan, $A^{1,4,5}$

Institut du Thorax, INSERM 1087 CNRS 6291, Nantes, France; ${ }^{2}$ Université de Nantes, Nantes, France; ${ }^{3}$ Institut de Transplantation Urologie-Nephrologie, INSERM 1064, Nantes, France; ${ }^{4}$ Hopital Nord Laennec, Nantes, France; ${ }^{5}$ Département Hospitalo-Universitaire (DHU) 2020, Nantes, France

Introduction: Asthma is a heterogeneous inflammatory disorder characterised with various endotypes leading to a series of clinical phenotypes. T-helper type 2 (Th2) response, mediated by IL-4, IL-5, and IL13 , predominates in most cases of asthma, but a Th17 response was also described, especially in severe cases in which a neutrophilic inflammation accompanies the canonical eosinophilia. IL-17A, the main cytokine produced by Th17 can induce a strong granulocytic airway infiltrate and airway hyperresponsiveness. We investigated in vivo the role of IL-17A in a model of asthma induced by house dust mite (HDM).

Methods: BALB/C mice were sensitised and challenged with house dust mite (HDM) extract. The respective effect of IL-17A, IL-13 and Polynuclear neutrophils (PNN) was determined after intranasal administration of IL-17A, IL-13, Ly6G neutralizing antibodies. Airway inflammation and hyperresponsiveness (AHR), bronchoalveolar lavage fluid cytokines levels, and lung $\mathrm{T}$ cell activation were assessed.

Results: In addition to AHR, asthmatic mice exhibit a huge influx of PNN and polynuclear Eosinophil (PNE) in lungs. This pulmonary infiltrate is associated with 
a mixed Th2 and Th17 activation with a strong expression and secretion of IL-13, IL-4, IL-17A. IL-17A neutralization totally abrogates AHR and reduces the PNN infiltrate. PNN depletion only a partially reduces AHR, suggesting a direct role of IL-17A on bronchial muscle contraction.
IL-13 neutralization had no effect. IL-17A but not IL-13 activated RHoA-MYPT1 signaling pathway leading to a strong bronchial contraction.

Conclusion: These results highlight an asthma model characterised by a mixed Th2/Th17 driven inflammation relevant to severe asthma. Asthmatic mice treated with anti-IL-17A neutralizing antibodies display impaired PNN recruitment and a restored lung function. Neutralization of IL-17A might be a useful therapeutic strategy to not only to reduce inflammation but also smooth muscle contraction. 


\section{Oral Abstract Session}

\section{OAS 31 - Epidemiology of asthma}

\begin{abstract}
178
Synergistic effect between low intake of vitamin $A$ and exposure to

environmental tobacco smoke on the risk of childhood asthma may be modified by GSTP1 polymorphism

Lee, S-Y ${ }^{1} ; \mathrm{Kim}, \mathrm{B}^{-\mathrm{S}^{2}}$; Kwon, J-W ${ }^{3} ; \mathrm{Kwon}, \mathrm{S}^{-\mathrm{O}^{4}}$; Oh,

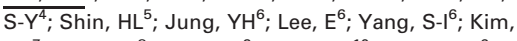

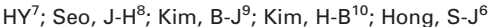
${ }^{1}$ Pediatrics, Hallym Sacred Heart Hospital, Anyang, Korea; ${ }^{2}$ Gangneung Asan Hospital, University of Ulsan College of Medicine, Gangneung, Korea; ${ }^{3}$ Seoul National University Bundang Hospital, Seongnam, Korea ; ${ }^{4}$ College of Human Ecology, Kyung Hee University, Seoul, Korea; ${ }^{5}$ Research Center for Standardization of Allergic Diseases, Asan Institute for Life Sciences, University of Ulsan College of Medicine, Seoul, Korea ; ${ }^{6}$ Childhood Asthma Atopy Center, Research Center for Standization of Allergic Diseases, Asan Medical Center, University of Ulsan College of Medicine, Seoul, Korea ; ${ }^{7}$ Kosin University Gospel Hospital, Busan, Korea ; ${ }^{8}$ Korea Cancer Center Hospital, Seoul, Korea; ${ }^{9}$ Inje University Haeundae Paik Hospital, Inje University College of Medicine, Busan, Korea ${ }^{10}$ Sanggye Paik Hospital, Inje University College of Medicine, Seoul, Korea
\end{abstract}

Background: Asthma is primarily an airway inflammatory disease, and the bronchial airways have been shown to be particularly susceptible to oxidant-induced tissue damage. The purpose of this study was to investigate the association with exposure to environmental oxidants such as environmental tobacco smoke (ETS), dietary antioxidants and susceptible genotypes on the risk of childhood asthma

Method: In this cross-sectional study, 1129 elementary school children aged 7-12 years old were included. Asthma symptoms and smoking history were measured using the ISAAC questionnaire and intake of vitamins $A$ (including retinol and $B$-carotene), $\mathrm{C}$, and $\mathrm{E}$ was measured by a semi-quantitative food frequency questionnaire (FFQ). GSTP1 polymorphisms were genotyped.

Results: ETS was significantly related with increased risk of current asthma symptoms (aOR, 2.48; 95\% CI, 1.29-4.76) and asthma diagnosis (aOR, 1.91; 95\% CI, 1.19-3.06). Association between dietary antioxidants and asthma symptoms was not found. However, the combination of ETS and low intake of vitamin A associated significantly and positively with asthma diagnosis (aOR, 2.23; 95\% CI, 1.10-4.54, $P$ for interaction $<0.05)$. Children with $\mathrm{AA}$ at nucleotide 1695 in GSTP1 who had been exposed to ETS and low intake of vitamin have an increased risk of asthma diagnosis
(aOR,4.44; 95\% CI,1.58-12.52) compared to those with $\mathrm{AG}$ or $\mathrm{GG}$ at this position and had not been exposed to two risk factors.

Conclusion: Low intake of vitamin $\mathrm{A}$ and exposure to ETS may affect the oxidative stress, which may in turn influence of the risk of asthma in children. These relationships may be modified by genetic susceptibility alleles of GSTP1.

\section{9}

Association between viral infections and asthma development is independent of virus type

Bønnelykke, $\mathrm{K}^{1}$; Vissing, $\mathrm{NH}^{1}$; Sevelsted, $\mathrm{A}^{1}$; Aniscenko, $\mathrm{J}^{2} ;$ Kebadze, $\mathrm{T}^{2} ;$ Johnston, $\mathrm{SL}^{2}$; Bisgaard, $\mathrm{H}^{1}$ ${ }^{1}$ Copenhagen Prospective Studies on Asthma in Childhood, Health Sciences, Danish Pediatric Asthma Center, University of Copenhagen, Copenhagen University Hospital, Gentofte, Denmark; ${ }^{2}$ Airway Disease Infection Section, MRC \& Asthma UK Centre in Allergic Mechanisms of Asthma, Centre for Respiratory Infection, National Heart \& Lung Institute, London, United Kingdom

Background: There is a strong focus on the role of specific viruses in possible mechanisms associating early respiratory infections to later asthma. However, several virus types can trigger similar early respiratory symptoms and it is possible that the important risk factors for asthma development are the underlying susceptibility to infections and the exaggerated reaction to such asthma triggers rather than the specific triggering agent. We aimed to study the role of individual viral infections in early life for development of asthma later in childhood.

Method: 313 children from the Copenhagen Prospective Studies on Asthma in Childhood $_{2000}\left(\right.$ COPSAC $\left._{2000}\right)$ high risk birth cohort were followed prospectively from birth to age 7 years. Viruses were analyzed from airway secretions sampled during episodes of troublesome lung symptoms during the first three years of life. We identified nine common respiratory viruses (respiratory syncytial virus, rhinoviruses, other picornaviruses, coronaviruses 229E\&OC43, parainfluenza viruses $1-3$, influenza viruses, human metapneumovirus, adenoviruses, and bocavirus). Asthma was determined clinically by age seven.
Results: In unadjusted analyses, all viruses identified during an episode of troublesome lung symptoms were associated with increased risk of asthma by age seven years. However, after adjustment for frequency of respiratory episodes viruses were no longer associated with asthma.

Conclusion: The number of respiratory episodes in the first years of life, but not the viral trigger, was associated with later asthma. This suggests that future research should focus on the susceptibility and exaggerated response to viral infections in general, rather than the specific triggering agent.

\section{0 \\ Maternal supplementary vitamin intake during lactation is positively associated with the cumulative incidence of bronchial asthma in their infants in Japan \\ Shinohara, $\mathrm{M}^{1,2}$; Matsumoto, $\mathrm{K}^{3}$ \\ Department of Pediatric Allergy, Shimoshizu National Hospital, Yotsukaido, Japan; ${ }^{2}$ Department of Pediatircs Kochi University, Nankoku, Japan; ${ }^{3}$ Department of Allergy \& Immunology, National Research Institute for Child Health \& Development, Okura, Japan}

Background: Vitamin is one of key antioxidant diets, and is thought to improve metabolism and diseases, including type 1 diabetes, infections, and bronchial asthma (BA). However, the effects are still controversial; early-life supplementation of vitamins $\mathrm{A}$ and $\mathrm{D}$, in water-soluble form seemed to increase the risk of allergic diseases, but those in peanut oil do not seem so (Kull I, JACI 2006), and early multivitamin supplementation increased the risk of BA (Milner JD, Pediatrics 2004). On the other hand, vitamin supplements use has been increasing in Japan. Therefore, we investigated whether prenatal and postnatal supplementary vitamin intakes by mothers influence the development of BA in their infants.

Method: Among a total 1436 parent-infant pairs, $1410(98 \%)$ infants with breast- and mixed-feeding and without supplementary vitamin intake were enrolled in this crosssectional survey in 2009. Supplementary vitamin intakes by parents during second and third trimester pregnancy and lactation, development of physician-diagnosed 
BA and so far were assessed by a self-writing questionnaire. An adjusted logistic regression model was analyzed by STATA software.

Results: The recovery rate was $97.2 \%$ (1436/1476). The cumulative incidence of BA was significantly increased in the infants with maternal supplementary vitamin intake during lactation (adjusted OR, 24.68; 95\% CI, 1.74-349.44) than in those without supplementary vitamin intake. Maternal supplementary vitamin intakes during second and third trimester pregnancy were not significantly associated with the cumulative incidence of BA.

Conclusion: Maternal supplementary vitamin in breast milk may modulate the development of BA in their infants. Adequate recommendation for vitamin supplements use would be needed.

\section{1 \\ Perinatal factors and sensitisation - a longitudinal study from birth to age 16 years \\ Bergström, $A^{1}$; Melén, $E^{1,2}$; Anderssson, $N^{1}$; Wickman, $\mathrm{M}^{1,2} ;$ Kull, $\mathrm{I}^{1,2,3}$ \\ ${ }^{1}$ Karolinska Institutet, Institute of Environmental Medicine, Stockholm, Sweden; ${ }^{2}$ Sachs' Children's and Youth Hospital, Södersjukhuset, Stockholm, Sweden; ${ }^{3}$ Department of Clinical Science and Education, Karolinska Institutet, Södersjukhuset, Stockholm, Sweden}

Background: Low birth weight and/or gestational age have been linked to an increased risk of asthma, but less is known about how these perinatal factors influence the development of sensitisation. We examined the association between birth weight, gestational age and sensitisation to airborne and food allergens in a longitudinal birth cohort followed from birth to age 16 years.

Method: Data was obtained from a Swedish birth cohort of 4089 children (BAMSE). Information on exposures and health characteristics were collected repeatedly through follow-up. In addition, blood samples for serological allergy testing were obtained at ages four, eight, and 16 years. Sensitisation was defined as $\geq 0.35 \mathrm{kU} / 1$ to airborne (Phadiatop) or food ( $\mathrm{f} \times 5$ ) allergens. Generalised estimating equations (GEE) assessed the overall and age-specific associations between birth weight $(\leq 2499$; 2500-2999; 3000-3499 (reference category); 3500-3999; $\geq 4000 \mathrm{~g}$ ) and gestational age (35-36; 37-38; 39-41 (reference category); 42-44 weeks) and sensitisation. In total, 3191 children born in week 35-44 were included in the present analyses.

Results: The mean birth weight in our study population was $3559 \mathrm{~g}$ (sd $507 \mathrm{~g}$ ) and the mean gestational age was
39.7 weeks (sd 1.5 weeks). In multivariate GEE analyses, high birth weight was associated with an overall decreased risk of sensitisation to airborne allergens (OR $0.82,95 \%$ CI $0.67-1.00$ for $\geq 4000 \mathrm{~g}$ compared to 3000-3499 g). Age-specific analyses showed that this association was present at ages eight (OR 0.71, 95\% CI $0.54-0.93$ ) and 16 years (OR $0.77,95 \% \mathrm{CI}$ $0.61-0.97$ ), but not at age four years (OR $0.95,95 \%$ CI $0.69-1.31)$. No statistically significant association was observed for low birth weight (OR 1.22, 95\% CI 0.761.95 for $\leq 2499 \mathrm{~g}$ compared to 3000 $3499 \mathrm{~g})$. There were no significant overall associations between gestational age and sensitisation to airborne allergens or between any of the perinatal factors and sensitisation to food allergens.

Conclusion: Our analyses of children from a population-based cohort followed from birth to adolescence indicate that perinatal factors may influence the development of sensitisation.

\section{2 \\ Ten year changes in symptoms of asthma and rhinitis by sensitisation status in a cohort of young adults \\ Garcia-Larsen, $\mathrm{V}^{1}$; Potts, $\mathrm{JF}^{1}$; Bustos, $\mathrm{P}^{2}$; Diaz, $\mathrm{PV}^{3}$; Amigo, $\mathrm{H}^{2}$; Oyarzun, $\mathrm{M}^{3}$; Rona, $\mathrm{RJ}^{4}$ \\ ${ }^{1}$ National Heart and Lung Institute, Imperial College London, London, United Kingdom; ${ }^{2}$ Department of Nutrition, University of Chile, Santiago, Chile; ${ }^{3}$ Institute of Biomedical Sciences, University of Chile, Santiago, Chile; ${ }^{4}$ Psychological Medicine, King's College London, London, United Kingdom}

Aim: To investigate the changes in asthma symptoms and rhinitis over a 10 year period within young adults according to skin prick test (SPT) reactions.

Methods: In 2001, a simple random sample of 1232 adults aged $22-28$ based on a sampling frame of births between 1974 and 1978 in Limache (Chile) was obtained for a cohort study on risk factors for asthma. 1193 subjects answered a lifestyle questionnaire and an adapted version of the European Community Respiratory Health Survey (ECRHS) questionnaire on asthma symptoms, and rhinitis. Bronchial hyperresponsiveness to methacholine (BHR) and SPT to eight allergens were assessed. Subjects were considered atopic if they had a positive reaction (wheal mean diameter $\geq 3 \mathrm{~mm}$ ) to any of 8 allergens tested. At follow-up (2011), 772 participants completed the questionnaires again. Generalised estimating equations (GEE) were used to estimate adjusted net changes in prevalence of respiratory symptoms and rhinitis by sensitisation status at baseline.

Results: A quarter of the participants were sensitised to at least one allergen in 2001.
At baseline, prevalence of wheeze in the last 12 months was 26.3 and 28.4 in males and females, respectively, with an overall net change per year of -0.37 ( $95 \%$ Confidence Interval -0.73 to -0.02$)$. Selfreported rhinitis in the last 12 months increased by 0.83 per year ( $95 \%$ CI 0.49 to 1.17). Those sensitised to cat fur, cockroach, grass and pollens, or weeds, were more likely to have wheeze in the last 12 months in 2011 (OR ranging from 1.74 to 2.04) after adjustment for wheeze in 2001. Having rhinitis in 2011 was consistently related to being sensitised to most allergens (OR ranging from 2.23 to 3.61) after adjustment for rhinitis in 2001 .

Conclusion: Self-reported symptoms of asthma tended to decrease over a period of 10 years, whilst rhinitis increased. Being sensitised to any of several allergens is a moderately strong risk factor for persistence of asthma symptoms and rhinitis.

183

Daily salt intake and asthma prevalence among Danish adults

Thuesen, $\mathrm{BH}^{1}$; Toft, $\mathrm{U}^{1}$; Jørgensen, $\mathrm{T}^{1,2,3}$; Linneberg, $\mathrm{A}^{1,2,4}$

${ }^{1}$ Research Centre for Prevention and Health, The Capital Region of Denmark, Glostrup, Denmark; ${ }^{2}$ Faculty of Health Science, University of Copenhagen, Copenhagen, Denmark: ${ }^{3}$ Faculty of Medicine, Aalborg University, Aalborg, Denmark; ${ }^{4}$ Department of Clinical Experimental Research, Glostrup University Hospital, Glostrup, Denmark

Background: Dietary changes associated with a more affluent Western lifestyle have been considered to possibly modify the risk of asthma. One characteristic of Western diets is high levels of dietary salt intakes and it has been suggested that salt may contribute to the raised prevalence of asthma seen in these countries. However, previous studies have been inconclusive, and we hypothesise that a potential association between salt and asthma may be influenced by the correlation between high daily salt intake and obesity, which also relates to asthma.

Methods: We included 3277 participants aged 18-69 years from the population based Danish Health2006 cohort. Estimated 24-h sodium excretion was calculated from measurements of creatinine and sodium in samples of spot urine. Asthma was defined as an affirmative answer to at least one of the following three questions: 'Within the last 12 months have you been woken by an attack of shortness of breath?', Within the last 12 months have you had an attack of asthma?' and 'Do you use any asthma medication currently?'. Logistic regression models gradually adjusted for age, sex, smoking status, and 
body mass index (BMI) was used to evaluate the association.

Results: Asthma was significantly associated with high levels of daily salt intake in age and sex adjusted regression models (odds ratio (OR): 1.08, 95\% confidence interval (CI): $1.01-1.16, \quad P=0.04)$. The association was also significant after fur- ther adjustment for smoking status (OR: $1.09,95 \%$ CI: $1.01-1.17, P=0.03)$. However, no significant association was found after adjustment for BMI (OR: 1.05, 95\% CI: $0.97-1.13 P=0.19$ ).

Conclusion: In this cross-sectional study including 3277 Danish adults, the prevalence of asthma was significantly associated with a high daily intake of salt, but the association was not significant after adjustment for BMI. Longitudinal studies are needed to determine the temporal relationship and interplay of salt intake, obesity, and asthma. 


\section{Oral Abstract Session}

\section{OAS 32 - AIT mechanisms}

\begin{abstract}
184
In situ characterisation of oral immune cells in birch-allergic patients: impact of the oral allergy syndrome and sublingual allergen immunotherapy

Mascarell, L ${ }^{1} ;$ Rak, $\mathrm{S}^{2}$; Worm, $\mathrm{M}^{3}$; Melac, $\mathrm{M}^{4}$; Soulie, $\mathrm{S}^{4}$; Lescailles, $\mathrm{G}^{5}$; Lemoine, $\mathrm{F}^{5}$; Jospin, $\mathrm{F}^{6}$; Paul, $\mathrm{S}^{7}$; Zeldin $\mathrm{RK}^{4}$; Baron-Bodo, $\mathrm{V}^{1}$; Moingeon, $\mathrm{P}^{1}$

${ }^{1}$ Research and Pharmaceutical Development, Stallergenes, Antony, France; ${ }^{2}$ Respiratory Medicine and Allergology, Sahlgrenska University Hospital, Göteborg, Sweden; ${ }^{3}$ Klinik für Dermatologie, Venerologie und Allergologie, Allergie-Centrum-Charité, CCM, Charité - Universitätsmedizin, Berlin, Germany; ${ }^{4}$ Global Clinical Development, Stallergenes, Antony, France; ${ }^{5}$ Groupe Hospitalier Pitié Salpêtrière, Paris, France; ${ }^{6}$ Faculté de Médecine Jacques Lisfranc, Saint Etienne, France; ${ }^{7}$ Laboratoire d'Immunologie et d'Immunomonitoring GIMAP, Faculté de Médecine Jacques Lisfranc, Saint Etienne, France
\end{abstract}

Background: Sublingual allergen immunotherapy (AIT) is efficient to treat type 1 respiratory allergies. Nonetheless, immune mechanisms involved remain to be further deciphered, particularly at the administration site. A detailed characterisation of human oral immune cells was undertaken and the role of specific subsets of antigenpresenting cells (APCs) was investigated in both healthy and birch-allergic individuals with or without Oral Allergy Syndrome (OAS), during AIT.

Method: Mapping of oral immune cells was conducted in gengiva from healthy donors. Subsequently, 572 adults suffering from birch pollen-induced rhinoconjunctivitis were randomized to receive daily placebo or 300IR sublingual solution of birch pollen extract starting 4 months before the pollen season. Oral mucosa biopsies were taken at baseline and at the end of the first period of treatment in 25 and 22 patients, respectively. 14 immune markers were analyzed by immunohistology.

Results: In all biopsies, limited numbers of eosinophils and mast cells were detected in the reticular layer of oral mucosa. Various subsets of APCs were identified in the oral mucosa, including CD207+ Langerhans and $\mathrm{CD} 11 \mathrm{c}+$ myeloid dendritic cells in the epithelium and lamina propria ( $L P$ ), as well as CD11b+ CD68+ macrophages in the $L P$ and reticular layer. We observed a significantly higher number $(P<0.01)$ of Langerhans cells in the mucosa of birchallergic patients without OAS in comparison with that of patients with OAS. After treatment, a significant increase in $\operatorname{IgE}+$ cells occurred in patients treated sublingually with the birch pollen extract solution. Co-localisation experiments confirmed that IgE bearing cells did not include CD207+ Langerhans cells but rather comprise $\mathrm{CD} 68+$ macrophages located in the $L P$.

Conclusion: Oral CD207+ langerhans cells may play a key role in the physiopathology of OAS. Oral CD68+ macrophages are likely critical in allergen capture via IgEdependent mechanisms in the course of sublingual AIT.

\section{5 \\ Suppressive activities of Tregs are maintained after discontinuation of treatment in epicutaneous and subcutaneous, but not sublingual immunotherapy}

Dioszeghy, $\mathrm{V}^{1}$; Mondoulet, $\mathrm{L}^{1}$; Dhelft, $\mathrm{V}^{1}$; Puteaux, $\mathrm{E}^{1}$; igouis, $\mathrm{M}^{1}$; Plaquet, $\mathrm{C}^{1}$; Dupont, $\mathrm{C}^{2}$; Benhamou, $\mathrm{P}-\mathrm{H}^{1}$ ${ }^{1}$ DBV Technologies, R\&D, Bagneux, France; ${ }^{2}$ Université Paris Descartes - Hôpital Necker, Paris, France

Background: The aim of allergen-specific immunotherapy is the induction of tolerance, most likely through the generation of regulatory $\mathrm{T}$ cells (Tregs). We compared the in vivo suppressive capacity of Tregs induced by epicutaneous (EPIT), sublingual (SLIT) or subcutaneous (SCIT) immunotherapy and their maintenance after end of treatment in a model of peanut-sensitised mice.

Method: Mice were sensitised orally to peanut protein extract (PPE) with cholera toxin and then treated for 8 weeks by EPIT, SLIT, or SCIT. CD25+CD4+ Tregs were isolated from spleen immediately after the end of treatment or 8 weeks after. Cells were then transferred into recipient PPEsensitised mice. The allergen-specific cytokine responses of splenocytes and the recruitment of eosinophils in esophagus after intensive oral exposure to PPE were measured in recipient mice and compared to sensitised not transferred mice and naïve mice.

Results: PPE-specific IL-5, IL-13, IL-10 and IFN- $\gamma$ production by splenocytes of mice transferred with Tregs isolated immediately after the end of treatment were significantly decreased compared to not transferred mice $(P<0.001)$, with no difference between EPIT, SLIT or SCIT. Mice that received Tregs isolated 8 weeks after the end of EPIT showed a similar decrease of cytokine production compared to not transferred mice $(P<0.01)$, whereas only IL-5 and IFN- $\gamma$ was decreased in mice that received Tregs isolated 8 weeks after SCIT $(P<0.05)$ and no cytokine was decreased in mice that received Tregs isolated 8 weeks after SLIT. The recruitment of eosinophils in esophagus after intensive oral exposure to PPE was decrease in mice transferred with Tregs isolated either immediately or 8 weeks after EPIT and SCIT, but not SLIT, compared to not transferred mice.

Conclusion: EPIT, SLIT and SCIT induced Tregs with suppressive activities. However, only EPIT and SCIT-induced Tregs offering protection from oral exposure and maintained after discontinuation of treatment.

\section{6}

Epicutaneous immunotherapy induces sustainable epigenetic modifications in peanut sensitised mice

Mondoulet, $\mathrm{L}^{1}$; Dioszeghy, $\mathrm{V}^{1}$; Puteaux, $\mathrm{E}^{1}$; Ligouis, $\mathrm{M}^{1}$; Plaquet, $\mathrm{C}^{1}$; Dhelft, $\mathrm{V}^{1}$; Dupont, $\mathrm{C}^{2}$; Benhamou, $\mathrm{P}-\mathrm{H}^{1}$ ${ }^{1}$ DBV Technologies, Bagneux, France; ${ }^{2}$ Hopital Necker Université Paris Descartes, Paris, France

Background: Epicutaneous immunotherapy (EPIT) consists of prolonged and uninterrupted application of the allergen on the skin, leading to rebalancing of Th2 allergic immune response. The purpose of this study was to investigate the implementation of epigenetic mechanisms underlying this therapeutic effect and its persistence. Method: BALB/c mice were orally sensitised to peanut and then treated by EPIT $(n=30)$ or sham $(n=30)$, with 30 naive mice serving as controls. Mice were sacrificed after 1, 2, 4, 6 and 8 weeks of EPIT and also 8 weeks after the end of EPIT. DNA methylation was analysed in spleen and blood samplings. Treg induction and profile of cytokine secretion were measured in spleen and mesenteric lymph nodes $(\mathrm{mLN})$ after 3 days of in vitro reaction.

Results: In the whole spleen of peanut-sensitised mice, EPIT increased methylation in 
the $\mathrm{CpG}$ islands of GATA-3, significantly vs Sham after 4, 6 and 8 weeks of treatment (respectively $64 \%, 58 \%$ and $59 \%$ vs $37 \%, 31 \%$ and $35 \%, P<0.05)$. This epigenetic modification was maintained 8 weeks after the end of EPIT (66\% vs $32 \%$ in Sham, $P<0.05)$. In the whole blood, the increase of methylation in the $\mathrm{CpG}$ islands of GATA-3 was observed only at the 8th week of EPIT $(62 \%)$ vs Sham $(31 \%$, $P<0.01)$. No modification was observed for Tbet transcription factor (in spleen and in blood. In parallel, the induction of Foxp3+ regulatory $\mathrm{T}$ cells (Tregs) was observed after 8 weeks of EPIT in the spleen and from the 4th week of EPIT until the end in the mLN. The induction is maintained over 8 weeks of treatment.

Conclusion: By prolonged and uninterrupted application of allergen on the skin, EPIT seems to be able to sustainably influence the immune response by modifying the DNA expression of Th2 transcription factor.

\section{7 \\ Induction of Arah2-specific memory B cells identified using a novel tetramer- based approach occurs early and transiently during peanut oral immunotherapy}

Patil, $S^{1}$; Ogunniyi, $A^{2} ; \mathrm{Ma}, \mathrm{A}^{3}$; Swenson, $\mathrm{T}^{3}$; Calatroni,

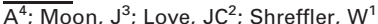

${ }^{1}$ Allergy and Immunology, Massachusetts General Hospital, Harvard Medical School, Boston, MA, United States; ${ }^{2}$ Massachusetts Institute of Techology, Chemical Engineering, Cambridge, MA, United States; ${ }^{3}$ Allergy and Immunology, Massachusetts General Hospital, Boston, MA, United States; ${ }^{4}$ Rho, Inc, Chapel Hill, NC, United States

Background: Peanut oral immunotherapy (PNOIT) increases tolerance peanut allergic patients and induces specific antibodies which might play a role in clinical protection. However, the mechanistic contribution of induced antibody clones to clinical tolerance in PNOIT is unknown. Hence, we identified affinity-selected antigen-specific B cells using a fluorescent tetramer of the purified peanut antigen, Arah2, to quantify and characterise PNOIT-induced humoral immunity.

Method: Peripheral mononuclear blood cells from PNOIT study participants $(n=22)$ as well as non-allergic controls $(n=7)$ were stained with Arah2 tetramers and analyzed by flow cytometry. From a subset of PNOIT patients $(n=3)$, paired heavy and light chain variable regions from tetramer-positive B cells were cloned by single cell PCR. Co-expression of paired heavy and light immunoglobulin chains were used to produce recombinant antibodies.
Results: The frequency of Ara$\mathrm{h} 2{ }^{+} \mathrm{CD} 19^{+} \mathrm{CD} 27^{+} \mathrm{IgM}^{-}$memory B cells per million $\mathrm{CD}^{+} 9^{+}$cells significantly increased during PNOIT (30.7 to 82.1, $P=0.01)$. This expansion of Arah2 ${ }^{+}$cells within the memory B cell compartment was transient, peaking at about 10 weeks during PNOIT $(P=0.01)$ and preceding the increase in specific antibody levels measured by ImmunoCAP assays. Of 118 cloned immunoglobulins, $34 \%$ were $\operatorname{IgM}$, $34 \% \operatorname{IgG}, 31 \% \operatorname{IgA}$ and $1 \%$ indeterminate. Increased somatic hypermutation and the presence of related clones sharing V-J segments and CDR3 homology support the enrichment for Arah2-specific clones. Of the recombinant antibodies successfully expressed from class-switched tetramerpositive B cells, 24 of the 29 antibodies were confirmed as Arah2-specific by ImmunoCAP and microarray analysis. Sequence analysis from Arah2-labelled B cells is notable for clonal convergence both between and among patients.

Conclusion: Using affinity selection with fluorescent B cell tetramers and single-cell BCR cloning, we have demonstrated early, transient expansion of circulating classswitched, antigen-specific memory B cells during PNOIT. This methodology will likely provide novel insights into humoral mechanisms of immunotherapy and allow us to more directly address the functional role of antigen-specific antibody induction in tolerance.

\section{8 \\ Differing homing properties of Tregs induced by epicutaneous compared to sublingual or subcutaneous immunotherapy in peanut-sensitised mice}

Dioszeghy, $\mathrm{V}^{1}$; Mondoulet, $\mathrm{L}^{1}$; Dhelft, $\mathrm{V}^{1}$; Ligouis, $\mathrm{M}^{1}$; Puteaux, $\mathrm{E}^{1}$; Plaquet, $\mathrm{C}^{1}$; Dupont, $\mathrm{C}^{2}$; Benhamou, $\mathrm{P}-\mathrm{H}^{1}$ ${ }^{1}$ DBV-Technologies, R\&D, Bagneux, France; ${ }^{2}$ Université Paris Descartes - Hôpital Necker, Paris, France

Background: Induction sites of regulatory $\mathrm{T}$ cells (Tregs) and immunotherapy routes influence homing properties which in turn impact their efficacy. In this study, we compared the expression of homing receptors for Tregs induced by epicutaneous (EPIT), sublingual (SLIT) or subcutaneous (SCIT) immunotherapy to assess their ability to respond to various allergy induced disorders.

Method: Mice were sensitised to peanut and divided into 4 groups: EPIT, SLIT, SCIT and not treated (Sham). After 8 weeks of treatment with $100 \mu \mathrm{g}$ of peanut protein extract, the proportion of Tregs in spleen, their expression of homing receptors, and the proportion of Tregs in related lymph nodes [inguinal (iLN) or mesenteric $(\mathrm{mLN})]$ was analyzed by flow cytometry.

Results: Whatever the method, spleen Foxp3+ Tregs increased with immunotherapy $(P<0.001$ compared to Sham $)$, more significantly with EPIT $(P<0.001$ compared to SLIT and SCIT). Regarding spleen Foxp3+ Tregs, the expression of CLA, a skin homing receptor, increased only with EPIT and SCIT. Consistently, only EPIT and SCIT increased Tregs in iLN $(P<0.01)$. For gut homing, only EPIT and SCIT induced higher expression of CCR9 in spleen Tregs, whereas all routes increased Tregs in mLN compared to Sham $(P<0.01)$. For lung homing, expression of CCR4, increased in Tregs with all routes. Interestingly, the CCR9+ Tregs induced by EPIT and SCIT expressed CLA but not CCR4. Significantly higher expression of CCR8, a welldefined Th2 homing receptor, CXCR3, a Th1 homing receptor and CCR6, a Th17 homing receptor was observed in EPITinduced Tregs compared to SLIT, SCIT and Sham.

Conclusion: EPIT appears to induce a larger repertoire of homing receptors on Tregs than SLIT, suggesting clinical applications in various organs. The induction of a population of CCR9+CLA+ Tregs further reinforces its potential interest in food allergy.

\section{9}

Langerhans cells are crucial in the skininduced tolerance to peanut

Yu, $\mathrm{X}^{1}$; Chen, $\mathrm{T}^{1}$; Feng, $\mathrm{H}^{1}$; Tian, $\mathrm{Y}^{1} ; \mathrm{Yao}, \mathrm{X}^{2} ; \mathrm{Li}, \mathrm{W}^{1}$ ${ }^{1}$ Dermatology, Xijing Hospital, Fourth Military Medical University, Xi'an, China; ${ }^{2}$ Allergy and Rheumatology, Institute of Dermatology, Chinese Academy of Medical Sciences, Nanjing, China

Background: Skin contact has been proposed for peanut sensitisation; however, we found previously that tolerance to peanut was achieved by applying peanut protein onto the intact skin of mice, which indicates that skin application of food allergen might be used as a prophylactic and/or therapeutic intervention for food allergy. The mechanism of the skin-induced tolerance is not clear yet. Langerhans cells (LCs) are the only dendritic cells present in the epidermis at the steady state, and have been shown to have negatively regulatory role in many allergic and inflammatory skin diseases. Here, we explore the role of LCs in the mechanism of the skin-induced tolerance to peanut.

Methods: Langerin-DTA mice that constitutively defect in Langerhans cells were utilised. Langerin-DTA mice and wide-type 
control were given 5 weekly cutaneous application of $1000 \mu \mathrm{g}$ complete peanut extract (CPE) on the shaved back skin, followed by 2 weekly intra-peritoneal injection of CPE to induce allergic reactivity to peanut. At various time-points, the immunologic responses and allergic clinical manifestations to allergens were examined. The number and phenotype of LCs, dermal dendritic cells and regulatory $\mathrm{T}$ cells in skin and regional draining lymph node of wide-type mice were analyzed after application of CPE onto intact skin.
Results: Skin application of CPE to structurally intact skin did not lead to allergic sensitisation to peanut in Langerin-DTA or wide-type mice. In wide-type mice, cutaneous allergen application blocked the subsequent induction of sensitisation including inhibiting i. p. sensitisation-induced CPEspecific IgE, IgG1 and $\operatorname{IgG} 2$ a production, suppressing of peanut anaphylaxis, and modulating the sensitisation-promoted cytokine production. However, there were almost no inhibitions on the sensitisation in Langerin-DTA mice. The number of migratory LCs and regulatory $\mathrm{T}$ cells increased in the regional draining lymph node of wide-type mice, and migratory LCs also showed an immature phenotype with low level of CD80 and CD86.

Conclusion: Langerhans cells play an important role in the skin-induced tolerance to peanut, suggesting that LCs might be a potential future target for the treatment of allergic disease. 


\section{Oral Abstract Session}

\section{OAS 33 - Urticaria and angioedema}

\begin{abstract}
190
Estrogens and $F 12$ gene mutations in women with antihistamine-resistant angioedema with normal C1-INH and negative family history

Bork, $\mathrm{K}^{1}$; Wulff, $\mathrm{K}^{2}$; Witzke, $\mathrm{G}^{1}$; Stanger, $\mathrm{C}^{1}$; Lohse, $\mathrm{P}^{3}$; Hardt, $\mathrm{J}^{4}$

${ }^{1}$ Department of Dermatology, University Medical Center, University Mainz, Mainz, Germany; ${ }^{2}$ Institute of Human Genetics, Ernst Moritz Arndt University, Greifswald, Germany; ${ }^{3}$ Institute of Laboratory Medicine and Human Genetics, Singen, Germany; ${ }^{4}$ Department of Medical Psychology and Medical Sociology, Johannes Gutenberg University Mainz, Mainz, Germany
\end{abstract}

Background: In women with sporadic recurrent angioedema with an unknown cause who are unresponsive to antihistamines and have normal $\mathrm{C} 1$ inhibitor activity and a negative family history of angioedema, it is unclear whether they have idiopathic angioedema or hereditary angioedema with normal $\mathrm{C} 1$ inhibitor, and what impact exogenous estrogens have on their angioedema.

Method: A cohort of 151 women was analyzed for F12 exon 9 mutations and for the influence of oral contraceptives, hormonal replacement therapies, and pregnancies on their angioedema.

Results: A total of 145 women had idiopathic angioedema unresponsive to antihistamines. Six women carried a F12 mutation and thereby had hereditary angioedema with F12 mutations. Among the women with idiopathic angioedema, 63 had never taken estrogens. There was no estrogen impact in 45 women, a moderate impact in 15, and a severe impact in 22 . The type and dose of estrogens did not differ in women with and without an estrogen impact. In 5 women, idiopathic angioedema disappeared after desogestrel use. Among the 6 women with hereditary angioedema with F12 mutations, angioedema symptoms occurred during 4 pregnancies, whereas no symptoms occurred during any of the 60 pregnancies in women with idiopathic angioedema.

Conclusion: Women with recurrent angioedema unresponsive to antihistamines may have idiopathic angioedema or, more rarely, hereditary angioedema with F12 mutations. Both conditions may be provoked or aggravated by exogenous estro- gens. In idiopathic angioedema, treatment with progestins may be helpful.

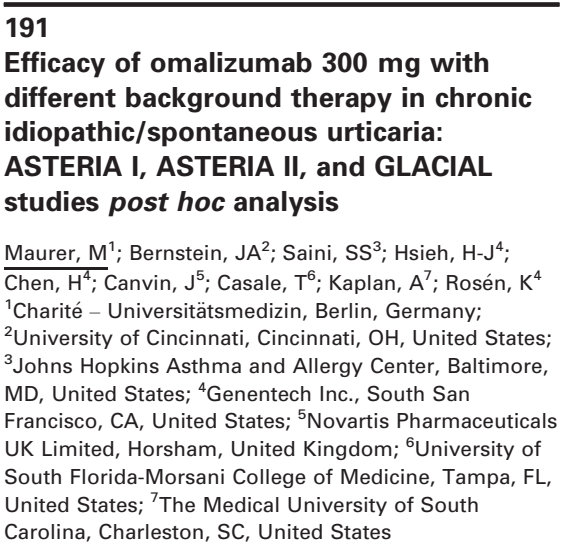

Background: Many patients with chronic idiopathic urticaria (CIU), also known as chronic spontaneous urticarial (CSU), remain symptomatic despite treatment using up to 4 times the approved dose of $\mathrm{H}_{1}$-antihistamines plus $\mathrm{H}_{2}$ - antihistamines and/or leukotriene receptor antagonists. Omalizumab has been shown to be effective in these patients, but it remains unclear, if treatment response is different on different background treatments.

Method: ASTERIA I and ASTERIA II were randomized, double-blind, placebocontrolled studies of omalizumab as addon therapy in CIU/CSU patients who remained symptomatic despite treatment with approved doses of $\mathrm{H}_{1}$-antihistamines. GLACIAL was a randomized, doubleblind, placebo-controlled study of add-on omalizumab in patients who remained symptomatic despite $\mathrm{H}_{1}$-antihistamines $\leq 4$ times approved dose plus $\mathrm{H}_{2}$-antihistamines and/or leukotriene receptor antagonists. We evaluated the efficacy of omalizumab $300 \mathrm{mg}$ every 4 weeks vs placebo in $\mathrm{CIU} / \mathrm{CSU}$ patients with different background therapies by pooling data from ASTERIA I and II and comparing with results from GLACIAL.

Results: At Week 12, the mean $(95 \%$ CI; $P$-value) weekly itch severity score (primary endpoint) was reduced from baseline by 5.28 points $(4.09,6.48 ; P<0.0001)$ in ASTERIA I/II $(N=319)$ in the pooled 300 -mg omalizumab groups and by 4.52 (3.08, 5.97; $P<0.0001)$ points in GLACIAL $(N=335)$ in the omalizumab 300-mg group relative to placebo. At Week $12,40.0 \%$ of patients in ASTERIA I/II and $33.7 \%$ of patients in GLACIAL in the omalizumab 300-mg groups were complete responders (Urticaria Activity Score over 7 days $=0$ ) compared with $6.9 \%$ and $4.8 \%$ of patients in the placebo group in ASTERIA I/II and GLACIAL, respectively (both $P<0.0001$ ). Results for other pooled ASTERIA I/II secondary endpoints were comparable with those observed in GLACIAL.

Conclusion: Regardless of current urticaria background therapy, omalizumab $300 \mathrm{mg}$ every 4 weeks had similar efficacy in CIU/ CSU patients.

\section{2 \\ Omalizumab normalises levels of high affinity lgE receptor-positive skin cells in patients with chronic spontaneous urticaria: a randomized, double-blind, placebo-controlled study}

Metz, $\mathrm{M}^{1}$; Staubach, $\mathrm{P}^{2}$; Bauer, $\mathrm{A}^{3}$; Brehler, $\mathrm{R}^{4}$; Gericke, $\mathrm{J}^{7}$; Kangas, $\mathrm{M}^{5}$; Ashton-Chess, $\mathrm{J}^{5}$; Jarvis, $\mathrm{P}^{5}$; Pan, $\mathrm{W}^{6}$; Georgiou, $\mathrm{P}^{7}$; Canvin, $\mathrm{J}^{7}$; Erpenbeck, $\mathrm{VJ}^{5}$; Maurer, $\mathrm{M}^{1}$ 'Dermatology and Allergy, Charité -

Universitätsmedizin, Berlin, Germany; ${ }^{2}$ Dermatology, University Medicine Mainz, Mainz, Germany; ${ }^{3}$ Dermatology, University Hospital Carl Gustav Carus, Technical University Dresden, Dresden, Germany;

${ }^{4}$ Dermatology, University Hospital Muenster, Muenster, Germany; ${ }^{5}$ Novartis Pharma AG, Basel, Switzerland; ${ }^{6}$ Novartis Pharmaceuticals Corporation, East Hanover, NJ, United States; ${ }^{7}$ Novartis Pharmaceuticals UK Limited, Horsham, West Sussex, United Kingdom

Background: Chronic spontaneous urticaria (CSU), including severe disease refractory to current therapies, shows a strong response to omalizumab, a humanised recombinant monoclonal anti-IgE antibody. The mechanism of action (MoA) of omalizumab in CSU has not been elucidated in detail. The MoA of omalizumab in CSU was explored, specifically effects on levels of high affinity IgE receptor positive $(\mathrm{FccRI}+)$ and $\operatorname{IgE}$ positive $(\mathrm{IgE}+)$ skin cells. Efficacy and PK/PD in CSU patients were also assessed.

Method: In a double-blind study, CSU patients aged 18-75 years were randomised to $300 \mathrm{mg}$ omalizumab $(n=20)$ or placebo $(n=10)$ s.c. every 4 weeks for 12 weeks (doses at Days 1, 29, and 57). Lesional and non-lesional skin was biopsied at Days 1, 
8, 29, 85 and 140. Biopsies from 10 untreated healthy volunteers (HVs) were used as a reference for histopathology data from treated patients. Efficacy was assessed in terms of UAS7.

Results: The omalizumab group showed a significantly greater decrease in UAS7 than the placebo group at Day 85 [mean (SD) -23.1 (12.94) vs -8.1 (14.45)], consistent with previous studies. CSU patients had higher levels of FceRI+ and IgE+ skin cells than HVs at Day 1. Levels of FceRI+ cells in both lesional and non-lesional skin declined significantly in omalizumab patients. Decreases were seen by Day 8 and continued to Day 85. Levels of $\operatorname{IgE}+$ skin cells also declined in omalizumab patients, but the change was not statistically significant (lesional skin: $P=0.135$; non-lesional skin: $P=0.058$ ). Levels of FceRI + and IgE+ cells in lesional and nonlesional skin in omalizumab patients at Day 85 had declined to levels seen in HVs. The placebo group showed no meaningful change in FceRI+ or IgE + cells. PK and PD of omalizumab were consistent with previous studies.

Conclusion: Omalizumab is effective in the treatment of CSU, and decreases FceRI+ cells in both lesional and non-lesional skin in CSU patients to levels seen in healthy individuals.

193

Association between disease-related discomfort, quality of life and somnolence in subjects with chronic urticaria treated with antihistamines

Petkova, EG; Rasheva, MY; Staevska, MT; Kralimarkova, TZ; Valerieva, AD; Dimitrov, VD; Popov, TA Clinical Centre of Allergology, Medical University of Sofia, Sofia, Bulgaria

Background: Chronic spontaneous urticaria (CSU) does not pose existential risks but causes substantial discomfort, so urticaria-related quality of life (URQoL) is an important outcome when assessing treatment options. Second generation antihistmaines (SGAH) are first line treatment, but difficult-to-treat cases may require higher than standard doses. The aim of our analysis was to determine how URQoL changed in the course of treatment with higher than standard doses of $\mathrm{AH}$ and whether it was affected by daytime somnolence.

Methods: The analysis included 101 patients (mean age 38 years, range 1968 years, 68 women, 33 men) from 2 different double-blind randomized clinical trials who did not respond to standard $\mathrm{AH}$ treatment. For the purpose of this study we assessed URQoL by specific questionnaire, CU-Q2oL, validated in Bulgarian (higher scores denoting worse QoL), and discomfort due to urticaria and daytime somnolence was measured on a $100 \mathrm{~mm}$ visual-analogue scale (lower values denoting worse dicomfort/somnolence): VASdisc and VASsomn respectively. Treatment was with up to $20 \mathrm{mg}$ levocetirizine $(n=51)$, $20 \mathrm{mg}$ desloratadine $(n=38)$ and $15 \mathrm{mg}$ levocetirizine $+50 \mathrm{mg}$ hydroxyzine at night $(n=12)$. Before/after treatment differences were correlated.

Results: Only $43 \%$ of the patients became symptomless at higher $\mathrm{AH}$ doses, but $90 \%$ of them reported less discomfort. Patients who took hydroxyzine at night had higher daytime somnolence than those on SGAH alone. The Pearson correlations between before/after treatment differences $(\Delta)$ in URQoL, VASdisc and VASsomn are presented in the following table:

\begin{tabular}{lll}
\hline $\begin{array}{l}\text { Patients } \\
(n=101)\end{array}$ & $\Delta$ URQoL & $\Delta$ VASsomn \\
\hline$\Delta$ VASdisc & $\begin{array}{l}-0.39 \\
(P<0.001)\end{array}$ & $0.41(P<0.001)$ \\
UURQoL & & $-0.22(P=0.028)$ \\
\hline
\end{tabular}

Conclusion: URQoL is inversely associated with both discomfort and daytime somnolence. This emphasizes the need to consider sedation when tailoring individual therapy in patients with difficult-to-treat CSU.

\section{4}

The common causes of urticaria in children admitted to the emergency room

Bezirganoglu, H; Arik Yilmaz, E; Soyer, O; Sekerel, BE; Sackesen, C

Department of Pediatric Allergy, Hacettepe University School of Medicine, Ankara, Turkey
Background: Urticaria is a common skin condition and is one of the major causes for the admissions to the emergency room. We aimed to identify the common causes of urticaria and determine the measures to prevent the recurrent admissions to the emergency room for urticaria.

Method: The urticaria code, L50, in the ICD system is used for the selection of children with the diagnosis of urticaria during 1 year (1 January-31 December 2013). Medical records concerning history and laboratory tests as well as direct phone call made with parents for the etiology of urticaria were noted. The causes of urticaria were classified as infections, drugs, foods, insect stings, blood products, vaccines and dyes. The diagnosis of infection was based on symptoms and physical examination whereas the diagnosis of food/ drug/venom allergy was based on the history of anaphylaxis, sIgE levels, skin prick tests and challenge tests.

Results: A total of 842 children had a diagnosis of urticaria, L50 code. When analyzed all children retrospectively we obtained data concerning urticaria from 465 children [5 years (2.0-9.1, median (IQR)]. The majority of the children $(88 \%)$ were referred for the first attack of urticaria and the remaining was recurrent urticaria $(11 \%)$ and chronic urticaria $(1 \%)$. The triggering factors as infections $(21 \%)$, drugs $(14 \%)$, foods $(8 \%)$, insect stings $(3 \%)$, blood products $(0.4 \%)$, vaccines $(0.4 \%)$, dye $(0.4 \%)$ and swimming pool $(0.4 \%)$ were reported as the cause of urticaria. The infections of upper respiratory, $(n=73)$, urinary $(n=14)$ and gastrointestinal systems $(n=8)$ and skin were defined. After the allergic work-up, the diagnosis of food allergy $(n=12)$, drug allergy $(n=9)$ and venom allergy $(n=2)$ were made in $4.9 \%$ of children with urticaria.

Conclusion: Urticaria is a distressing disease due to fear of life-threatening condition during childhood and an important cause of admissions to emergency room. Infection is determined as the most frequent cause of urticaria followed by food and drug allergies as second and third causes of urticaria in childhood. 


\section{Oral Abstract Session}

\section{OAS 34 - Ocular allergy}

\section{5 \\ Imaging-based airborne allergy diagnosis with conjunctival provocation test}

Astvatsatourov, $\mathrm{A}^{1,2}$; Zadoyan, $\mathrm{G}^{1}$; Mösges, $\mathrm{R}^{1}$ Institute of Medical Statistics, Informatics and Epidemiology, University Hospital of Cologne, Cologne, Germany; ${ }^{2}$ Experimental Ophthalmology, University Hospital of Cologne, Cologne Ophthalmological Reading and Image Analysis Center, Cologne, Germany

Background: The conjunctival provocation test (CPT) is a diagnostic procedure, established in testing during the non-active season for airborne allergens causing allergic rhinoconjunctivitis. It has been thoroughly evaluated with regard to its precision and compared with nasal provocation tests. The CPT is performed by topical application of allergen solutions of increasing concentrations onto the patient's conjunctiva. Nowadays, the CPT is used for evaluation of allergic rhinoconjunctivitis.

Method: Allergic reaction to CPT is estimated by numerical severity score. The diagnosis takes into account subjective and objective allergic symtoms. It is documented with high-resolution macro-photos of the patient eyes. Applicability of the developed analysis is shown in the pre-seasonal dose-finding study in 159 patients, which was carried out with birch pollen allergic patients. Following data acquisition and evaluation methods are suggested for validation and standardisation of the CPT:

1. Fast and easy acquisition of the macro-images of eyes for study documentation and for after-study estimations.

2. Digital imaging of eyes - automated and computing-objectified evaluation of the allergic reaction, which includes conjunctiva segmentation, reddening and vessels analysis.

3. Simple and robust statistical methods, which outline the severity of an allergic reaction.

Results: Saturation effect is shown for the therapy efficacy in respect to the medication dose. The superior effect of the highest medication doses over the lowest dose was found to be statistically significant $(P<0.01$ in F-test $)$.

Conclusion: The developed CPT analysis chain is an appropriate evaluation method of the airborne allergy diagnosis in drug registration trials.
196

Nasal and ocular response after allergen, histamine and hypertonic saline nasal provocation in subjects with seasonal allergic rhinitis

Kalogjera, $\mathrm{L}^{1}$; Tomljenovic, $\mathrm{D}^{1}$; Bukovec, $\mathrm{Z}^{2}$; Bokulic, $\mathrm{A}^{3}$; $\overline{\text { Baudoin, } T^{1}}$

${ }^{1}$ ORL Head and Neck, University Hospital Centre 'Sestre Milosrdnice', Zagreb, Croatia; ${ }^{2}$ Endo Lab, University Hospital Centre 'Sisters of Mercy', Zagreb, Croatia, ;

${ }^{3}$ Endo Lab, University Hospital Centre 'Sestre

Milosrdnice', Zagreb, Croatia

Background: Allergens and irritants applied into the nose of the allergic individual induce nasal and ocular symptoms due to the release of inflammatory mediators and neurokininins. Naso-ocular reflex may be induced through stimulation of different populations of sensory receptors. The aim of the study was to compare naso-ocular reflex response in patients with seasonal allergic rhinitis after different methods of specific and non-specific nasal provocation.

Method: A group of 27 patients, age 1860 years, both gender, monoallergic to grass or ragweed pollen, after informed consent, were submitted to baseline nasal provocation with distilled water mist out of season. Patients with acute rhinitis, chronic rhinosinusitis, nasal polyps, significant septal deformity, and those taking topical or systemic medication which may interfere with the response to provocation were excluded. On the consecutive day, 500 I.U. of lyophilized allergen per nostril in water solution was applied to both inferior turbinates with micropipette. Next day $80 \mathrm{mcg}$ of histamine per nostril were applied in the same fashion. More than $48 \mathrm{~h}$ after histamine, same type of provocation was done with $2 \%$ hypertonic saline (HTS). Before each provocation, acoustic rhinometry (AR), visual analog scale (VAS) subjective scores of nasal and ocular symptoms and nasal lavage were done. After each provocation Schirmer test was done $5 \mathrm{~min}$ after provocation bilaterally, VAS scores and nasal lavages were done 15 min after each challenge. Nasal lavages and tears collected on filter Schrimer strips were analysed for tryptase, eosinophil cation protein (ECP) and substance $\mathrm{P}$ (SP).

Results: There is a significant correlation between Schirmer scores between allergen, histamine and hypertonic provocation
$(P<0.01)$. SP concentrations in tears correlated significantly after histamine and hypertonic saline provocations $(P<0.05)$ and baseline, but not after allergen provocation. Ocular itch was related to SP levels and nasal itch with tryptase. Tryptase was detectable $(<1 \mathrm{ng} / \mathrm{ml})$ in less than half of the patients after low dose allergen nasal provocation.

Conclusion: Volume of tears after nasal allergen challenge is significantly correlated with the magnitude of lacrimation after histamine and hypertonic saline challenge. Concentrations of SP in tears after nonspecfic challenges correlate significantly. Priming after allergen challenge significantly modifies naso-ocular reflex.

197

Conjunctival provocation tests: a predictive factor for patients' seasonal allergic rhinoconjunctivitis symptoms

Eichel, A; Kruse, K; Gerwin, E; Astvatsatourov, A; ShahHosseini, K; Mösges, R

Institute for Medical Informatics, University of Cologne, Cologne, Germany

Background: No parameters currently exist that can reliably predict the impact of preseasonal immunotherapy on the symptoms occurring during the season. The purpose of our studies was to prove a correlation between pre-seasonal conjunctival allergen challenge and co-seasonal primary clinical endpoints using the total combined score, i.e., a combination of symptom and medication score, as the primary outcome parameter.

Method: Twelve weeks before both the birch and the grass pollen seasons, two separate prospective, double-blind, randomized, controlled studies were conducted followed by post-trial observations for each study during the active season. In the studies, patients reacting to conjunctival allergen challenge were treated with sublingual immunotherapy tablets containing either birch/alder or grass pollen allergoids.

Results: In all, 158 patients were included in the grass and 160 in the tree pollen study; of these, 100 and 109 patients, respectively, took part in the post-trial 
observations. When comparing patients with and without a positive reaction in the final conjunctival allergen challenge, the results revealed a significant difference in the total combined score (birch: $P<0.001$; grass: $P=0.039$ ). The same applied to the rescue medication score $(P=0.005$; $P=0.025)$. A significant difference regarding the rhinoconjunctivitis symptom score was shown in the grass pollen study $(P=0.002)$, and the difference of well days was significant in the tree pollen study $(P=0.049)$.

Conclusion: When comparing patients based on their reaction to allergen challenge before and after immunotherapy, each study leads to similarly significant results. Therefore, conjunctival allergen challenge can be used effectively as a parameter to predict patients' allergic rhinoconjunctivitis symptoms during the season.

\section{8 \\ Aeroallergen vensitisation and prevalence of asthma, allergic rhinitis and eczema in children with vernal keratoconjunctivitis attending Red Cross War Memorial Children's Hospital}

Naidoo, $\mathrm{S}^{1}$; Levin, $\mathrm{M}^{2}$; Tinley, $\mathrm{C}^{2}$; Pollock, $\mathrm{T}^{2}$

'Paediatrics, Red Cross Children's Hopsital, Cape Town, South Africa; ${ }^{2}$ Red Cross Children's Hopsital, Cape

Town, South Africa

Background: Vernal Keratoconjunctivitis (VKC) is a severe inflammatory disease of the conjunctiva, with complex inflammatory pathways involving $\operatorname{IgE}$ and non $\operatorname{IgE}$ mechanisms. Studies have suggested that there are marked differences in the clinical expression of VKC in Africa with less atopy than in European and Asian cohorts.

Aim: Describe the prevalence of allergic sensitisation to common aeroallergens as well as the prevalence of asthma, allergic rhinitis, and eczema in a cohort of children attending Red Cross Children's Hospital, Cape Town, South Africa.

Method: A cross sectional descriptive study where patients under 13 years had

A diagnosis of VKC confirmed by an ophthalmologist.

Completed a questionnaire regarding atopic diseases and symptoms.

A physical examination.

Sensitisation evaluated by skin prick testing (SPT) to common aeroallergens.

Results: 168 patients were enrolled, mainly male $(74.5 \%)$, black African $(71 \%)$ and ranging from 19 months to $12 \frac{1}{2}$ years.

$54.2 \%$ (89) patients had co-morbid allergic rhinitis while other atopic diseases were less prevalent. $7.6 \%$ (13) of our patients had active eczema while 14\% (24) had signs of chronic eczema. 39\% (67) of patients had a history of non-specific wheeze with only $17 \%$ (30) previously diagnosed with asthma and less with active disease.

Significant aeroallergens on SPT were HDM $(55.8 \%)$, grass $(33.3 \%)$ and cockroach $(18.5 \%)$ followed by cat $(12.5 \%)$, $\operatorname{dog}(10.7 \%)$, tree pollens $(5.9 \%)$ and mould $(3.5 \%)$.

Conclusion: Our patient cohort has an incidence of allergic sensitisation and atopic disease higher than unselected cohorts in South Africa and VKC cohorts in previous African studies. Our VKC cohort more closely resembles the quoted VKC data from Europe and Asia than the earlier African VKC studies. The major aeroallergens are HDM, grass and cockroach. The prevalence of asthma and eczema found in this study is similar to non selected South African subjects, but the prevalence of allergic rhinitis is much higher.

\section{9}

\section{Ocular contact allergy: experience in 143} patients

\section{Ale, IS}

Unit of Cutaneous Allergy, Department of Allergology \& Department of Dermatology, Republic University of Uruguay, Montevideo, Uruguay

Background: Ocular allergy is a multifaceted clinical problem. The external eye is exposed to a large number of environmental, cosmetic, pharmacologic and occupational allergens, and the prevalence of external eye diseases related to the prolonged use of ophthalmic medications (blepharoconjunctivitis medicamentosa) and contact lens wear is increasing. Differential diagnosis may be difficult because the clinical patterns of eye reactions are restricted and different clinical entities may share similar clinical signs and symptoms. In addition, the pathogenic mechanisms are often intricate.

Method: This is a retrospective study of 143 patients who presented with persistent or recurrent blepharitis or blepharoconjunctivitis as a primary or secondary complaint.

Patients underwent skin testing and, when indicated in vitro studies and usage test as part of the workup. Patch testing was performed with the extended international standard series, a specially designed series containing the most frequent allergens in preparations for ophthalmic use in Uruguay, allergens from cosmetics, corticosteroids and preservatives, as well as with the ophthalmic preparations and cosmetics used by the patient.
Results: A relevant contact allergy was found in 94 of 143 patients $(66 \%)$. Forty four patients $(31 \%)$ had atopic eczema, of whom 21 also had contact allergy.

The most frequent allergens involved were eye medications -especially antibiotics and preservatives- and cosmetics, including fragrances, preservatives and nail products. Other sources of contact sensitivity include metal and rubber allergens, corticosteroids, mydriatic agents and topical drugs for glaucoma, dust mites and animal dander.

Conclusion: Ocular contact allergy is not uncommon. Allergens form eye medications and cosmetics were the most frequent sensitisers. Patients with persistent blepharitis and/or conjunctivitis should be referred for allergological evaluation.

The rational use of ophthalmic preparations and other allergenic substances should decrease the incidence of allergic contact reactions.

200

Systematic allergy investigation of patients with red eyes

Munk, SJ ${ }^{1}$; Garvey, $\mathrm{LH}^{2}$; Heegaard, $\mathrm{S}^{1,3}$; Hansen, $\mathrm{KS}^{2,4}$; Skov, $\mathrm{L}^{5}$; Zachariae, $\mathrm{C}^{5}$; Menné, $\mathrm{T}^{5}$; Julian, $\mathrm{HO}^{3}$; Halkjær, $\mathrm{L}^{2}$; Johansen, $\mathrm{JD}^{6}$

${ }^{1}$ Department of Neuroscience and Pharmacology, Eye Pathology Institute, University of Copenhagen,

Denmark; ${ }^{2}$ Allergy Clinic, Gentofte Hospital, University of Copenhagen, Denmark; ${ }^{3}$ Department of Ophthalmology, Glostrup Hospital, University of Copenhagen, Denmark; ${ }^{4}$ Department of Pediatrics, Herlev Hospital, University of Copenhagen, Denmark; ${ }^{5}$ Department of Dermato-Allergology, Gentofte Hospital, University of Copenhagen, Denmark; ${ }^{6}$ Department of Dermato-Allergology, National Allergy Research Centre, Gentofte Hospital, University of Copenhagen, Denmark

Introduction: Inflammation of the conjunctiva presenting as 'red eyes' is a common patient complaint. Determining the underlying cause is often difficult as differential diagnoses are numerous. Allergic causes may be easily overlooked as the diagnosis relies on specialised investigation and testing for relevant allergens. Using a multidis- 
ciplinary approach we wanted to determine the prevalence of relevant allergy in patients with 'red eyes', where initial ophthalmological consultation did not result in a definite diagnosis.

Methods: A multidisciplinary cooperation was established between ophthalmologists, allergologists, dermatologists and pediatricians. The investigation algorithm was based on clinical presentation. Patients with eye symptoms only, or eye and skin symptoms, underwent dermatological investigation comprising patch testing with European baseline series, eye drops (oph- thalmological preparations) including ingredients and skin prick tests with inhalant allergens and the patients' own ophthalmological products. Patients with eye and airway symptoms were reviewed by allergologists and underwent skin prick tests and specific IgE analysis for relevant allergens and lung function tests. Children were reviewed by pediatric allergologists. Multidisciplinary conferences were held regularly and patients were reviewed and cross-referred if relevant.

Results: In total 58 patients, [21 males (aged 20-75) and 37 females (aged 3-95)] with 'red eyes' were included consecutively over 1 year. In 21 patients $(36 \%)$ a relevant allergy was found, diagnosing contact allergy in 15 patients ( 9 perfume, 3 phenylephrine, 3 others) and IgE-mediated inhalant allergies (pollen, molds, dustmite, animal dander) in 6 patients. In 37 patients $(64 \%)$ no allergy was found, and of these 10 patients were diagnosed with rosacea.

Conclusion: Multidisciplinary cooperation and systematic investigation resulted in identifying relevant allergy in $36 \%$ of patients presenting with 'red eyes'. 


\section{Oral Abstract Session}

\section{OAS 35 - Pediatric Epidemiology}

\begin{abstract}
201
Monitoring the impact of cow's milk hypersensitivity on children and their families with the FLIP questionnaire over time
\end{abstract}

Mikkelsen, $\mathrm{A}^{1}$; Mehlig, $\mathrm{K}^{1}$; Borres, $\mathrm{MP}^{2}$; Björkelund, $\mathrm{C}^{3}$; Oxelmark, $\mathrm{L}^{4}$; Lissner, $\mathrm{L}^{1}$

${ }^{1}$ Department of Publich Health and Community Medicine/Public Health Epidemiology, Sahlgrenska Academy, University of Gothenburg, Gothenburg, Sweden; ${ }^{2}$ Department of Pediatrics, Sahlgrenska Academy, University of Gothenburg, Gothenburg, Sweden; ${ }^{3}$ Department of Publich Health and Community Medicine, Sahlgrenska Academy, University of Gothenburg, Gothenburg, Sweden; ${ }^{4}$ Division of Nursing, Deaprtment of Neurobiology, Care Sciences and Society, Karolinska University Hospital and Karolinska Institutet, Stockholm, Sweden

Background: Cow's milk hypersensitivity $(\mathrm{CMH})$ in children typically changes over time and many develop tolerance. However, the perceived challenge on daily life might remain. We sought to measure the change in impairment in affected families following the progression of the $\mathrm{CMH}$ using a new Food hypersensitivity family ImPact questionnaire (FLIP) and the generic Swedish Parental Stress Questionnaire (SPSQ).

Method: Families of children with $\mathrm{CMH}$, who participated in the original construction and validation of the FLIP (1), were re-approached 6 months later for followup. Change in impairment over time was assessed by paired sample t-test between both occasions in the FLIP's total and subscales' scores. The analysis was stratified by the progression of $\mathrm{CMH}$, i.e. out-grown vs persistent $\mathrm{CMH}$ at follow up for comparison. Longitudinal change was also assessed using the generic SPSQ.

Results: Perceived impairment in families with children who had outgrown $\mathrm{CMH}$ $(n=20)$ decreased as measured by the FLIP's total score $(P=0.0005)$ and for 2 subscales; Health \& Emotions $(P=0.0005)$ and Everyday Life $(P=0.0005)$ but no changes were registered in the Nutrition subscale. The impairment in the group with persistent $\mathrm{CMH} \quad(n=57)$ was unchanged at follow up except for an increased impact on Everyday Life $(P=0.001)$. The SPSQ did not measure differences in perceived stress regardless of the progression of the $\mathrm{CMH}$.

Conclusion: Currently affected families experienced sustained impact of $\mathrm{CMH}$ in daily life. Despite development of tolerance, no longer affected families revealed sustained impact on nutritional aspects. The FLIP is sensitive and effective in measuring impairment caused by food hypersensitivity and change over time following the progression of $\mathrm{CMH}$.

Keywords: Children and family, cow's milk hypersensitivity, food hypersensitivity, impact on daily life, longitudinal validity.

\section{2 \\ Breast milk fatty acids in relation to sensitisation at $\mathbf{6}$ months of age \\ Rosenlund, $\mathrm{H}^{1,2}$; Mie, $\mathrm{A}^{1} ;$ Alm, JS ${ }^{1,3}$ \\ Department of Clinical Research and Education, Karolinska Institutet, Södersjukhuset, Stockholm, Sweden; ${ }^{2}$ Department of Clinical Nutrition and Dietetics, Karolinska University Hospital, Stockholm, Sweden; 'Sachs' Children's and Youth Hospital, Stockholm, Sweden}

Introduction: Several cross-sectional studies indicate that an anthroposophic lifestyle reduces the risk of allergy in children. The anthroposophic lifestyle is characterised by home deliveries, organic diet, restricted use of vaccinations, as well as by other features that may affect the allergy risk. Previous studies have reported an association between breast milk fatty acid composition and allergy in children.

The aim of this study was to investigate the relation between breast milk fatty acids and development of sensitisation at 6 months of age among infants from families with different lifestyles.

Methods: The prospective birth cohort ALADDIN includes 550 children from anthroposophic, partly anthroposophic and non-anthroposophic families in Sweden. ALADDIN aims at elucidating the role of specific lifestyle factors that could mediate the lower allergy prevalence in anthroposophic children.

For this study, 245 mature breast milk samples were collected when the infant was 2 months old. The samples were analysed for fatty acid composition by gas chromatography and flame ionisation detection (GC-FID). Allergen-specific IgE levels against 7 common allergens were obtained from blood samples collected at age 6 months. Logistic regression was used for the statistical analyses.
Results: Almost 40 different fatty acids were detected, although not in every sample. The most common fatty acid was Oleic acid (C18:1(n-9)).

An inverse association was observed between Vaccenic acid (C18:1(n-7) trans) and sensitisation at 6 months of age (OR, highest vs lowest quartile, OR $0.19,95 \%$ CI $0.05-0.74, P$ for trend 0.029). On the opposite, Arachidic acid (C20:0) was positively associated with sensitisation at 6 months (OR 4.98, 95\% CI 1.17-21.1, P for trend 0.05$)$. The statistical model used for these analyses has so far been adjusted for Lifestyle.

Further analyses are planned to study associations between breast milk fatty acids and sensitisation at ages 12 and 24 months.

\section{3}

Incidence and risk factors of infections in early childhood - the COPSAC 2010 clinical birth cohort

Andersen, JIM; Vissing, NH; Bisgaard, $\mathrm{H}$ Copenhagen Prospective Studies on Asthma in Childhood, Health Sciences, University of Copenhagen \& Danish Pediatric Asthma Center, Copenhagen University Hospital, Gentofte, Denmark

Background: There is a considerable variation in susceptibility to infections among otherwise healthy children, but little is known about actual incidence of infections and risk factors explaining this variation.

Aims: To describe incidence of infections and risk factors in the first 2 years of life in a clinical birth cohort study.

Material and methods: The Copenhagen Prospective Study on Asthma in Childhood $_{2010}($ COPSAC 2010$)$ is an unselected birth cohort of 700 children with close clinical follow-up throughout childhood. Health symptoms and infections were registered using daily diary cards and reviewed by a research doctor at 6-monthly follow-up visits at the research clinic. The data are described by median and interquartile range [IQR]. Risk factor analysis was done by Poisson regression.

Results: At age 0-2 years, the children $(n=608)$ suffered 14 [9-20] upper respiratory tract infections, $1[1-1]$ lower respiratory tract infection, 4 [2-6] gastrointestinal infections and 12 [8-17] episodes of fever. 
Risk for all infections increased with male sex $(\mathrm{IRR}=1.06, P=0.01)$, older siblings (IRR $=1.11, P=0.01)$, maternal level of education $(\mathrm{IRR}=1.18, P=0)$, maternal age $\quad(\mathrm{IRR}=1.006, \quad P=0.03)$, gestational age $\quad(\mathrm{IRR}=1.00, \quad P=0.02) \quad$ and high income (IRR $=1.02 P=0.03)$. There was a trend suggesting from daycare attendance (IR $=1.00, P=0.07)$, but no effect from smoking during pregnancy $(\mathrm{IRR}=0.95$, $P=0.31)$ or duration of breastfeeding $(\mathrm{IRR}=1.00, P=0.17)$.

Conclusions: Incidence of childhood infections is highly variable between children. Older siblings, sex, gestational age, maternal age at delivery and educational level and high income are risk factors significantly associated with increased disease incidence, but they explain only a minor fraction of the inter-individual variation. It appears that environmental exposures only have minor impact and the major determinants of common childhood infections remains unknown.

\section{4 \\ Longitudinal and cross sectional assessment of allergic diseases and social influences differ: results from GINIplus and LISAplus}

Klümper, $C^{1} ;$ Krämer, $U^{1} ;$ Röder, $S^{2} ;$ Lehman, $I^{2} ;$ Bauer, $\mathrm{C}^{3} \mathrm{P}^{3}$; Berdel, $\mathrm{D}^{4}$; von Berg, $\mathrm{A}^{4}$; Borte, $\mathrm{M}^{5,6}$; FilipiakPittrofff, $\mathrm{B}^{7} ;$ Heinrich, $\mathrm{J}^{8} ;$ Herbarth, $\mathrm{O}^{9} ;$ Hoffmann, $\mathrm{U}^{3}$; Koletzko, $\mathrm{S}^{7}$; Link, $\mathrm{E}^{1}$; Schaaf, $\mathrm{B}^{10}$; Hoffmann, $\mathrm{B}^{1,11}$; for the GINIplus; LISAplus Study Groups

${ }^{1}$ Epidemiology, IUF-Leibniz Research Institute for Environmental Medicine, Düsseldorf, Germany; ${ }^{2}$ Department of Environmental Immunology, Helmholtz Centre for Environmental Research - UFZ, Leipzig, Germany; ${ }^{3}$ Department of Pediatrics, Technische Universität München, Munich, Germany; ${ }^{4}$ Children's Department, Research Institute, Marien-Hospital, Wesel, Germany; ${ }^{5}$ Department of Pediatrics, University of Leipig, Leipzig, Germany; ${ }^{6}$ Municipal Hospital St. Georg, Leipzig, Germany; ${ }^{7}$ Dr. von Hauner Children's Hospital, Ludwig Maximilians University Munich,

Munich, Germany; ${ }^{8}$ Helmholtz Centre Munich, German Research Centre for Environmental Health, Institute of Epidemiology, Munich, Germany; ${ }^{9}$ Faculty of Medicine, Environmental Medicine and Environmental Hygiene, University of Leipzig, Leipzig, Germany; ${ }^{10}$ Praxis fur Kinder- und Jugendmedizin, Bad Honnef, Germany;

${ }^{11}$ Medical Faculty, Heinrich-Heine University of

Düsseldorf, Düsseldorf, Germany

Background: Cross-sectional estimates of self-reported life time prevalence of allergic diseases are widely used to determine the burden of disease and their association with influencing factors. However recall bias, assumptions about missing data and reverse causation might crucially affect the validity of results.

The aim of this study was to compare life-time prevalence of eczema, hay fever or asthma until the age of 10 years and its association with parental education as an indicator for socio-economic status (SES) using a longitudinal and a cross-sectional approach.
Method: We used questionnaire data from children participating in the birth cohort studies GINIplus and LISAplus. At each follow up parents were asked whether a physician had diagnosed their child with eczema, hay fever or asthma since the last follow up examination. Life-time prevalences were estimated longitudinally by

1. combining the period-prevalences from different follow-up examinations counting a 'yes' if any of the follow-ups were 'yes' and 'no' if all were 'no' and by

2. cumulative incidence estimates from Kaplan Meier method and

3. cross-sectionally by using the question 'Has your child ever had eczema (hay fever/ asthma)?' in the 10-year questionnaire.

Associations with SES were investigated by using logistic regression and Cox regression respectively. Models were adjusted for sex and parental allergy.

Results: Estimates for life-time prevalence were different: $38.2 \%$ (method 1), $27.0 \%$ (method 2) and $18.3 \%$ (method 3) for eczema, $10.4 \%, 7.6 \%$ and $5.9 \%$ for asthma and $18.1 \%, 13.9 \%$ and $10.5 \%$ for hay fever, respectively. Whereas Cox regression revealed no association between eczema and SES [hazard ratio 1.03 (95\% CI $0.96-$ $1.11)]$, the cross-sectional odds ratio [1.12 (95\% CI 0.99-1.27)] showed a higher prevalence in better educated. The odds ratio for the simple longitudinal estimate (method 1$)$ was in the opposite direction [0.89 (95\% CI 0.81-0.98)] indicating a lower prevalence in better educated. Results for asthma and hay fever were similar.

Conclusion: Cross-sectional estimates and simple longitudinal estimates of life time prevalence are biased. Bias such as recall bias and patterns of missing values might differ between educational groups leading to biased estimates of the association.

\section{5 \\ Multiple sensitisation to food allergens predisposes to develop sensitisation to inhaled allergens in infants with early- onset atopic dermatitis: evidence from the ORCA prospective cohort}

Amat, $\mathrm{F}^{1,2,3}$; Boutmy-Deslandes, $\mathrm{E}^{4}$; Desseaux, $\mathrm{K}^{4}$; $\overline{\text { Nemni, }} A^{1}$; Bourrat, $E^{5}$; Sarahoui, $F^{1}$; Pansé, $1^{5}$; Fouere, $\mathrm{S}^{5}$; Bagot, $\mathrm{M}^{5}$; Just, $\mathrm{J}^{1,2,3}$

${ }^{1}$ Allergologie Pédiatrique, AP-HP - Hôpital d'Enfants Armand Trousseau, Université Paris 6, Paris, France; ${ }^{2}$ Equipe EPAR, Institut Pierre Louis d'Epidémiologie et de Santé Publique, INSERM UMR S 1136, Paris, France; ${ }^{3}$ Equipe EPAR, Institut Pierre Louis d'Epidémiologie et de Santé Publique, Sorbonne Universités, UPMC Université Paris 6, UMR S 1136, Paris, France; ${ }^{4}$ Service de Biostatistiques et Informatique Médicale, AP-HP, Hôpital Saint Louis, Université Paris 7, Paris, France; ${ }^{5}$ Service de Dermatologie, AP-HP, Hôpital Saint Louis, Université Paris 7, Paris, France

Background: Atopic dermatitis (AD) is common in children, however some AD phenotypes are at risk to develop asthma and are associated with poor prognosis. The objective of this study was to assess predictive markers associated with the development of sensitisation to inhaled allergens in a cohort of infants with earlyonset AD.

Method: Children were prospectively followed up both by a dermatologist and an allergo-pulmonologist, at the age of 6,12 and 18 months, and then annually until the age of 6 years, and standardised biological tests were performed during the follow-up. Allergic sensitisation was defined as a positive skin prick test and specific IgE $\geq 0.35 \mathrm{kIU} / 1$ towards a common allergen and multiple sensitisation as an allergic sensitisation to more than one allergen. Blood eosinophilia was defined as a concentration $\geq 470$ eosinophils $/ \mathrm{mm}^{3}$ and elevated total $\mathrm{IgE}$ as a concentration $\geq 45 \mathrm{kU} /$ 1.

Results: 229 infants were included, with an average age of 6.5 months $( \pm 2.7) ; 134$ $(58.5 \%)$ were boys. At baseline, $26.2 \%$ of the children had a blood eosinophilia and $36.9 \%$ elevated total IgE. These two markers remained significantly high during the follow-up when they were elevated at baseline. Sensitisation to food allergens decreased over time (from 58 to $49 \%$ ) whereas sensitisation to inhaled allergens increased (from 17 to $67 \%$ ). Initial multiple sensitisation to food allergens was found to be the most predictive marker associated with the risk of sensitisation to inhaled allergens at the age of 6 [OR 3.72 $(1.68-8.30) ; P<0.001-$ HR $1.90 \pm 0.13$; $P<0.001]$

Conclusion: Recognition of this particular phenotype of early-onset AD associated with a multiple sensitisation to food allergens, at high risk of sensitisation to inhaled allergens, is essential to a proper patient management.

\section{6 \\ Examining the change over time in the odds of passing an oral food challenge (OFC) test using the Cork Southampton calculator}

Hourihane, J; Donnelly, J; Dunn Galvin, A Department of Paediatrics and Child Health, University College Cork, Cork, Ireland

Background: Currently OFCs are the gold standard investigation for diagnosing allergic disease, however they are not risk free, are costly, and are time-consuming. Furthermore, tolerance may develop while waiting for an OFC, resulting in a negative challenge that could have been avoided. The Cork Southampton calculator predicts OFC outcome with up to $96 \%$ accuracy, 
and may be used longitudinally to demonstrate this trend towards tolerance. We aimed to retrospectively assess how the odds of passing an OFC changed over time while patients awaited testing.

Method: A retrospective chart review in the paediatric department of a single centre was carried out, including patients with egg and peanut allergies. Patient data were used with the calculator to determine the changes in odds of passing an OFC over a period of 4 years. Overall trends were then extrapolated from these results to demonstrate how their allergies had changed during the study period.

Results: Overall, 41 egg and 42 peanut allergic patients met inclusion criteria. Predictably, many egg allergic patient's odds of passing an OFC improved while awaiting the test, but this was not seen in peanut allergic children.

Conclusion: A child's chances of passing an OFC improves over a 4 years period for egg allergy but not for peanut allergy.
Scheduleing priority may be given to patients if their allergy is likely to be resolving. Patients may be followed using the Cork Southampton calculator to delineate who does or does not appear to be developing tolerance. Overall, waiting times for OFCs and heathcare costs could be reduced by decreasing the number of OFCs scheduled and performed. 


\section{Oral Abstract Session}

\section{OAS 36 - Assessing control in primary care}

207

Does nasal nitric oxide correlate with obesity in allergic and nonallergic rhinitis?

Baccioglu, $\mathrm{A}^{1}$; Kalpaklioglu, $\mathrm{AF}^{1}$; Gungor, $\mathrm{O}^{2}$ Immunology and Allergic Diseases, Kirikkale University, Kirikkale, Turkey; ${ }^{2}$ Pulmonary Diseases, Kirikkale University, Kirikkale, Turkey

Background: Nasal nitric oxide (nNO), a well-known marker of airway inflammation, is rarely evaluated in rhinitis of different aetiology. As its association with obesity is unknown, we aimed to investigate the serum leptin levels and the effect of changes in body mass index (BMI) on nNO levels in patients with allergic (AR) and nonallergic rhinitis (NAR).

Method: Patients were enrolled on the basis of chronic nasal symptoms. Total nasal symptom scores (TNSS), BMI ( $\mathrm{kg} /$ $\mathrm{m}^{2}$ ), allergy skin prick tests, and serum total $\operatorname{IgE}$ (T.IgE) were evaluated. Serum leptin levels $(\mathrm{ng} / \mathrm{ml})$ were measured using solid-phase-enzyme-immunoassay (DIAsource Immunoassays S.A. Louvin-la-Neuve, Belgium). Nasal NO (parts per billion: ppb) was measured with transnasal method at a flow rate of $5 \mathrm{ml} / \mathrm{s}$, using a chemiluminescence's analyzer $\left(\mathrm{NIOX}^{\circledR}\right.$ analyzer; Aerocrine $^{\circledR}$, Sweden).

Results: A total of 412 patients $(64.8 \% \mathrm{~F}$, mean age: $27 \pm 15$ years) including AR $(n=281) \quad$ and NAR $(n=131)$ were enrolled. nNO levels of allergic group were higher than NAR group $(377 \pm 343 \mathrm{ppb}$, and $257 \pm 331 \mathrm{ppb}, \quad P=0.004)$. Serum leptin levels were similar in allergic and nonallergic rhinitics $(3.16 \pm 3.20 \mathrm{ng} / \mathrm{ml}$, and $\quad 3.40 \pm 3.70 \mathrm{ng} / \mathrm{ml}$, respectively, $P=0.09)$. The mean BMI of the whole group was $23.66 \pm 4.28 \mathrm{~kg} / \mathrm{m}^{2}$, and frequency of obesity (BMI $\geq 25 \mathrm{~kg} / \mathrm{m}^{2}$ ) was $31.3 \% \quad(N=129)$, which was similar between rhinitis groups. Mean $\mathrm{T}$. $\mathrm{IgE}$ and nNO levels, gender distribution, asthma comorbidity, mean TNSSs, and duration as well as severity of rhinitis were not different between obese and non-obese subgroups of AR and NAR. When only obese ones were included, patients with AR had higher mean age, TNSS, and nNO values, and NAR group had higher serum leptin levels compared to each other. There was no correlation between $\mathrm{nNO}$ levels and BMI, however nNO levels were correlated with T.IgE levels in patients with AR+obese, and serum leptin levels in patients with NAR+obese $(r=-0.203, \quad P<0.001$, and $r=0.102, P=0.04)$.

Conclusion: No direct relation was detected between obesity and nNO. However, inflammation mediated by adipose tissue and designated by leptin is involved in nonallergic nasal inflammation, despite the higher nNO values in allergic rhinitis.

\section{8}

Biomarkers in exhaled breath and the nose: relation with allergy and asthma symptoms in primary school children

De Prins, $S^{1,2}$; Marcucci, $F^{3}$; Sensi, $L^{3} ;$ Van de Mieroop, $\mathrm{E}^{4}$; Nelen, $\mathrm{V}^{4}$; Nawrot, $\mathrm{T}^{5}$; Schoeters, $\mathrm{G}^{1,2}$; Koppen, $\mathrm{G}^{1}$ ${ }^{1}$ VITO, Mol, Belgium; ${ }^{2}$ University of Antwerp, Antwerp, Belgium; ${ }^{3}$ University of Perugia, Perugia, Italy; ${ }^{4}$ Provincial Insitute for Hygiene, Antwerp, Belgium;

${ }^{5}$ Hasselt University, Hasselt, Belgium

\section{Background: Our aim was}

1. To estimate the prevalence of asthma and allergy symptoms in a population of 282 primary school children in the city of Antwerp, and

2. To investigate if these symptoms correlate with biomarker measurements in a subgroup of 130 children.

Method: In the HEAPS study (Health Effects of Air Pollution in Antwerp Schools), 282 primary school children (6 to 12 years old) in two schools in the city of Antwerp filled out an ISAAC type questionnaire regarding asthma (asthma, wheezing, dry cough), rhinitis (runny nose, hay fever), and eczema (itchy rash, eczema) symptoms. Noninvasive biomarkers for oxidative stress and inflammation were analyzed in a subgroup of 130 children. We measured fractional exhaled nitric oxide (FeNO) and exhaled breath condensate (EBC) markers including $\mathrm{pH}, 8$-isoprostane and IL-1 $\beta$. Nasal specific immunoglobulin $\mathrm{E}$ in the nose for five common aeroallergens was assessed, and nasal concentrations of IL-8, eosinophilic cation protein (ECP), and tryptase were analyzed. Regression analysis taking into account confounding factors such as age, sex, and BMI was done to estimate biomarker changes in response to allergy and asthma symptoms.

Results: Frequencies of asthma symptoms ranged from $6.5 \%$ (wheezing with exercise) to $31.4 \%$ (dry cough) in the whole study group, and from $4.7 \%$ (wheezing with exercise) to $27.3 \%$ (dry cough) in the biomarker subgroup. Prevalence of rhinitis symptoms ranged from $9.8 \%$ (hay fever) to $36.8 \%$ (runny nose) in the whole group, and from $10.2 \%$ (hay fever) to $35.9 \%$ (runny nose) in the subgroup. For eczema symptoms, frequencies were between $22.1 \%$ (itchy rash) and $30.2 \%$ (eczema) for the whole group and between $25.6 \%$ (itchy rash) and $34.4 \%$ (eczema) for the subgroup. Recent wheeze was associated with FeNO and nasal tryptase, with increased values $(95 \% \mathrm{CI})$ of $139 \%(56-265 \%)$ and $214 \% \quad(18-740 \%)$, respectively. Rhinitis symptoms were associated with nasal tryptase, which increased by $100 \%(17-242 \%)$. FeNO and nasal tryptase were linked with the diagnosis of airway allergy using the Rhinostick, with respective increases of $128 \%(70-205 \%)$ and $250 \%(73-609 \%)$.

No correlations between biomarkers and questionnaire data on eczema were observed.

Conclusion: Rhinitis symptoms were associated with nasal tryptase levels while wheeze was associated with FeNO and nasal tryptase. Both biomarkers were furthermore influenced by atopy status, reflected by nasal sIgE.

209

Utility of allergen-specific IgE measurements for supporting the diagnosis of hen's egg, cow's milk and wheat allergy

Sato, $\mathrm{S}^{1}$; Ogura, $\mathrm{K}^{1}$; Takahashi, $\mathrm{K}^{1}$; Kutsuwada, $\mathrm{K}^{1}$; Sato, $\mathrm{Y}^{2}$; Ebisawa, $\mathrm{M}^{1}$

${ }^{1}$ Clinical Research Center for Allergy and Rheumatology, Sagamihara National Hospital, Kanagawa, Japan; ${ }^{2}$ Department of Biostatistics, Clinical Research Center Chiba University, Chiba, Japan

Background: Food allergy (FA) is increasing among children, with the three most common food allergenic sources being hen's egg (HE), cow's milk (CM) and wheat (W). Allergen-specific IgE (sIgE) measurements by IMMULITE $^{\circledR} 2000$ 3 gAllergyтм (3g) were evaluated for their utility in diagnosis of FA in subjects with suspected FA disorders.

Method: Serum samples were collected from 1164 patients with suspected FA (age: 
0-9 years; HE: $n=257, \mathrm{CM}: n=301$ and $\mathrm{W}: n=606$ ), who initially visited our hospital between May 2010 and December 2011. sIgE for egg white (EW), ovomucoid (OVM), CM, casein (C), W and gluten $(\mathrm{G})$ were measured using $3 \mathrm{~g}$ and ImmunoCAP ${ }^{\circledR}$ (CAP) sIgE assays. Serum samples were drawn within 6 months from the initial visit. Final diagnosis of FA was based on the positive oral food challenge (OFC) and convincing histories of FA-related disorders within 6 months of serum collection. Predicted probability curves (PCs) were plotted using the results from logistic regression analysis.

Results: Of 1164 patients, 239 children were diagnosed with FA (HE: $n=90, \mathrm{CM}$ : $n=68, \mathrm{~W}: n=81)$. Regarding $3 \mathrm{~g}$ results, the sIgE levels in patients with FA were significantly higher than in food-tolerant patients $(P<0.01)$. Positive Predictive Values (PPV) were significantly increased with correlation to increasing sIgE levels and age. The majority of patients 4 years of age or older, whose sIgE levels were lower than the detection levels, were food-tolerant. The $95 \%$ PPV as determined by $3 \mathrm{~g}$ PCs were obtained for $\mathrm{CM}$ and $\mathrm{W}$, but could not be calculated for EW and OVM. The 95\% PPVs were different for different allergens and allergenic components (CM: $\left.240\left(\mathrm{IU}_{\mathrm{A}} / \mathrm{ml}\right), \mathrm{C}: 331, \mathrm{~W}: 148, \mathrm{G}: 117\right)$. The $95 \%$ PPV for $\mathrm{W}$ was determined by $3 \mathrm{~g}$, but could not be determined by CAP. Conclusion: Measurements of sIgE to HE, $\mathrm{CM}$ and $\mathrm{W}$ and their individual allergenic components may be used as a tool to facilitate the diagnosis of FA in subjects with suspected allergic disorders.

210

Assessing allergic rhinitis symptom control: results from a digital survey conducted during EAACI 2013

Price, $D^{1}$; Papadopoulos, $N^{2}$; Scadding, $\mathrm{G}^{3}$; Rasp, $\mathrm{G}^{4}$. Bousquet, $\mathrm{J}^{5}$

${ }^{1}$ Department of General Practice and Primary Care, University of Aberdeen, Aberdeen, United Kingdom:

${ }^{2}$ Allergy Research Center, University of Athens, Athens, Greece; ${ }^{3}$ The Royal National Throat Nose and Ear Hospital, London, United Kingdom; ${ }^{4}$ University Clinic of Ear Nose and Throat Disease, Salzburg, Austria;

${ }^{5}$ Clinique des Maladies Respiratoires, Hopital Arnaud de Villeneuve, Montpellier, France

Background: Allergic rhinitis (AR) is poorly controlled in many patients, who continue to experience nasal and ocular symptoms $^{1}$ even on multiple therapies. ${ }^{2}$ However, exactly what is meant by "control'? A simple way to assess AR control is needed; a common language for physicians and patients to more effectively manage this disease. The aim of this survey was to assess how physicians

1. measure AR control,

2. perceive the control status of patients and

3. regard the usefulness of a simple visual analogue scale (VAS) to gauge control.

Method: 307 EAACI 2013 delegates completed an iPAD survey. Delegates were asked to

1. indicate how many AR patients they saw per week during the season,

2. assess the proportion of patients they considered to be well-, partly- and un-controlled,

3. communicate how they gauged control ( $>1$ answer permitted) and

4. assess how useful they would find a VAS as a method of gauging control.

Results: Respondents reported seeing 46.8 (SD 68.5) AR patients/week on average during the season. They considered that AR was controlled, partly controlled and uncontrolled in $38.7 \%$ (SD 24.0), 34.2\% (SD 20.2 and $20.0 \%$ (SD 16.34) of patients, respectively. Delegates reported assessing disease control in many different ways, including symptom severity (74\%), frequency of day- and night-time symptoms $(67 \%)$, activity impairment $(57 \%)$, respiratory function monitoring $(40 \%)$ and incidence of exacerbations $(50 \%) .91 \%$ of delegates felt that a VAS was a useful tool to assess disease control.

Conclusion: Physicians assessed AR control in many different ways and considered that over $50 \%$ of their patients had sub-optimally controlled disease. Almost all physicians considered the VAS a useful tool to assess AR control. Such a tool would enable control assessment in a simple and consistent way and should improve the way this disease is perceived and managed.

References:

1. Bousquet P. Int Arch Allergy Immunol 2013;160:393-400.

2. Price D. presented at EAACI 2013.

211

Participation and satisfaction of an online educational research project for parents of children with food allergy

Contreras-Porta, $\mathrm{J}^{1}$; Ruiz-Baqués, $\mathrm{A}^{2}$; Sáinz de la Maza, $\mathrm{E}^{3}$; Capel, $\mathrm{F}^{4} ;$ Ariño, $\mathrm{M}^{5}$; Zorrozua, $\mathrm{A}^{6}$

${ }^{1}$ Hospital Universitario La Paz, Madrid, Spain; ${ }^{2}$ Ideas en Salud, Barcelona, Spain; ${ }^{3}$ Educadies, México DF, Mexico; ${ }^{4}$ AEPNAA, Murcia, Spain; ${ }^{5}$ Immunitas Vera, Barcelona, Spain; ${ }^{6}$ Elikalte, Bilbao, Spain

Background: Food allergy is the most frequent cause of anaphylactic reactions and it is estimated to affect $7 \%$ of children in Spain.

Objective: To describe the participation level and satisfaction of an online educational program for parents of children aged $<12$ years old who are at high risk of anaphylaxis due to food allergy.Parents of children with food allergy have shown interest by participate actively in educational online program extra activities: discussion forums and video chats. Participants refer very high satisfaction with the educational online program, which shows the value to implement these initiatives and integrate them into healthcare system.

Method: In the context of an educational research project comparing an online vs inperson educational program, we analyzed the impact among parents of the online version carried out on a virtual classroom based on Moodle. Several media types were used, such as educational videos, games, and storytelling on how to avoid allergens, treat allergic reactions and how to use the Epinephrine Auto-Injector, labelling, cooking, shopping and relations with healthcare professionals and school, among others. Online discussion forums moderated by physicians and expert patients, and video chat courses were also established.

Results: Of a total of 392 participants, 207 attended the online program during February 2013 and 185 the in-person during November 2012. Of those 207 attending the online educational program, $67 \%$ (139) visited the online forums: $106(76.2 \%)$ visited the forums up to 25 times, and 33 $(23.8 \%)$ more than 26 times during the 2 weeks course.

The video chat leaded by a physician (allergist) had 57 attendants $(27.5 \%)$, the second and third leaded by expert patients had $38(18.3 \%)$, and $33(15.9 \%)$ attendants respectively.

Regarding satisfaction; $85 \%$ of online participants rated the course between 8 and 10 points $(0$ considered not satisfied and 10 very satisfied). Stressing that $45 \%$ rated it with 10 .

Conclusion: Parents of children with food allergy have shown interest by participate actively in educational online program extra activities: discussion forums and video chats. Participants refer very high satisfaction with the educational online program, which shows the value to implement these initiatives and integrate them into healthcare system. 